\title{
A RULE-BASED CONTROLLER BASED ON SUCTION DETECTION FOR ROTARY BLOOD PUMPS
}

\author{
by \\ Antonio Luiz S. Ferreira \\ Master in Electrical Engineering \\ Universidade Federal do Maranhão - Brazil, 1998 \\ Submitted to the Graduate Faculty of \\ the School of Engineering in partial fulfillment \\ of the requirements for the degree of \\ Doctor of Philosophy
}

University of Pittsburgh

2007 


\section{UNIVERSITY OF PITTSBURGH SCHOOL OF ENGINEERING}

This dissertation was presented

by

Antonio Luiz S. Ferreira

It was defended on

July 20th 2007

and approved by

J. Robert Boston, PhD, Department of Electrical and Computer Engineering James F. Antaki, PhD, Department of Bioengineering C. C. Li, PhD, Department of Electrical and Computer Engineering Luis F. Chaparro, PhD, Department of Electrical and Computer Engineering Patrick Loughlin, PhD, Department of Electrical and Computer Engineering S. Shorff, PhD, Department of Bioengineering

Dissertation Director: J. Robert Boston, PhD, Department of Electrical and Computer Engineering 


\title{
ABSTRACT \\ A RULE-BASED CONTROLLER BASED ON SUCTION DETECTION FOR ROTARY BLOOD PUMPS
}

\author{
Antonio Luiz S. Ferreira, PhD \\ University of Pittsburgh, 2007
}

A new rule-based control system for rotary ventricular assist devices (rVADs) is proposed. The control system is comprised of two modules: a suction detector and a rule-based controller. The suction detector can classify pump flow patterns, based on a discriminant analysis (DA) model that combines several indices derived from the pump flow signal, to make a decision about the pump status. The indices considered in this approach are frequency, time, and time-frequency-domain indices. These indices are combined in a DA decision system to generate a suction alarm.

The suction detector performance was assessed using experimental data and in simulations. Experimental results comprise predictive discriminant analysis (classification accu-

racy: $100 \%$ specificity, $93 \%$ sensitivity on training set and $97 \%$ specificity, $86 \%$ sensitivity on test set) of the detector and descriptive discriminant analysis (explained variance) of the DA model. To perform the simulation studies, the suction detector was coupled to a cardiovascular-pump model that included a suction model. Simulations were carried out to access the detector performance, under different physiological conditions, i.e., by varying preload and the contractility state of the left ventricle. To verify its robustness to noise, simulations were carried out to verify how the accuracy of the detector is affected when increasing levels of noise are added to the pump flow signal.

The rule-based controller uses fuzzy logic to combine the discriminant scores from the DA model to automatically adjust the pump speed. The effects on controller performance 
of symmetric or asymmetric membership output sets and the dimension of the rule base were evaluated in simulations. The same parameter changes, i.e., preload and contractility, were used to assess the control system performance under different physiologic scenarios in simulations. The proposed control system is capable of automatically adjusting pump speed, providing pump flow according to the patient's level of activity, while sustaining adequate perfusion pressures and avoiding suction. In addition, the control system performance was not adversely affected by noise until SNR was less than $20 \mathrm{~dB}$, which is a higher noise level than is commonly encountered in flow sensors used clinically for this type of application. 


\section{TABLE OF CONTENTS}

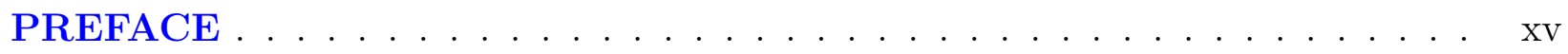

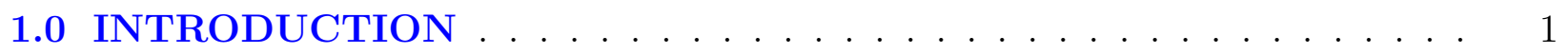

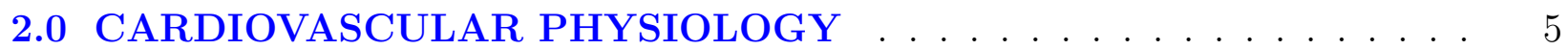

2.1 Heart and Circulation . . . . . . . . . . . . . 5

2.2 The Cardiac Cycle . . . . . . . . . . . . . . . . . . 7

2.3 The pressure-volume relationship . . . . . . . . . . . . . . 8

2.4 Properties of vessels . . . . . . . . . . . . . . . . . . . . 10

2.5 MODELS OF THE CARDIOVASCULAR SYSTEM $\ldots \ldots \ldots \ldots$

2.5 .1 Ventricular models . . . . . . . . . . . . . . . . . . . . . . 12

2.5.2 Afterload Models . . . . . . . . . . . . . . . . . . . . 13

3.0 FUZZY LOGIC CONTROLLER . . . . . . . . . . . . . . . . . . 15

3.1 Fuzzy Sets . . . . . . . . . . . . . . . . . . . . . 15

3.1 .1 Properties of fuzzy sets $\ldots \ldots \ldots \ldots \ldots$

3.1 .2 Operations on Fuzzy Sets . . . . . . . . . . . . . . . 18

3.2 Fuzzy Relation . . . . . . . . . . . . . . . . . . . . . . . . . 19

3.2 .1 Fuzzy If-Then statements . . . . . . . . . . . . . . . . 19

3.2 .2 Composition . . . . . . . . . . . . . . . . 20

3.2 .3 Linguistic Variables . . . . . . . . . . . . . . . . . . . 21

3.3 Fuzzy Control Systems . . . . . . . . . . . . . . . . . . . . . . . . 21

3.3 .1 Design of a Fuzzy Logic Controller . . . . . . . . . . . . . . . . . 22

4.0 THE CARDIOVASCULAR MODEL . . . . . . . . . . . . . . 25

4.1 The cardiovascular model . . . . . . . . . . . . . . . . . . . 26 
4.1 .1 Modeling the ventricle . . . . . . . . . . . . . . . . 27

4.1 .2 Modeling the valves . . . . . . . . . . . . . . . . . . . . . 29

4.1 .3 State Equations . . . . . . . . . . . . . . . . . . . . . 31

4.2 Model Validation . . . . . . . . . . . . . . . . . . . . . . . . . . 31

4.2 .1 Input Impedance $\ldots \ldots \ldots$

4.2 .2 Fitting Human Data . . . . . . . . . . . . . . . . . . 35

4.3 The coupled cardiovascular-pump model . . . . . . . . . . . . . . . 36

4.3.1 Suction element model . . . . . . . . . . . . . . . . . . . . . . 37

4.4 State equations . . . . . . . . . . . . . . . . 38

4.5 Open Loop response . . . . . . . . . . . . . . . . . . . . . . 40

4.5 .1 Step response . . . . . . . . . . . . . . . . . 40

4.5 .2 Ramp response . . . . . . . . . . . . . . . . . . . . . . . 41

4.5.3 Comparison with experimental data . . . . . . . . . . . . . . 42

5.0 SUCTION DETECTION PROBLEM IN ROTARY BLOOD PUMPS 45

5.1 Feature Extraction of Pump Flow . . . . . . . . . . . . . . . . . 46

5.1 .1 The window length issue $\ldots \ldots \ldots \ldots \ldots$

5.1 .2 Frequency based suction indices . . . . . . . . . . . . . 51

5.1 .3 Time based indices . . . . . . . . . . . . . . . . 56

5.1 .4 Time-frequency based index . . . . . . . . . . . . . 58

5.2 The Decision System . . . . . . . . . . . . . . . . . . . . . 61

5.2.1 The Discriminant Analysis Method _. . . . . . . . . . . . . 61

5.2 .2 Classification rule . . . . . . . . . . . . . . . . . 66

5.2 .3 Misclassification Cost . . . . . . . . . . . . . . . . . . 68

5.2 .4 Methods . . . . . . . . . . . . . . . . . . . . . . 69

5.3 Experimental Results . . . . . . . . . . . . . . . . . . . 72

5.3 .1 Data description . . . . . . . . . . . . . . . . . 73

5.3 .2 Minimum number of samples . . . . . . . . . . . . . . . 78

5.3.3 The two-group classification problem $\ldots \ldots \ldots \ldots . \ldots 79$

5.3.3.1 Descriptive Discriminant Analysis . . . . . . . . . . . . 79

5.3.3.2 Predictive Discriminant Analysis _. . . . . . . . . . 79 
5.3.4 Classifying the test set for the two-group problem . . . . . . . . . 82

5.3.5 The three-group classification problem . . . . . . . . . . . . . 82

5.3.5.1 Descriptive Discriminant Analysis . . . . . . . . . . . 83

5.3.5.2 Predictive Discriminant Analysis . . . . . . . . . . . 85

5.3.6 Classifying the test set for the three-group problem . . . . . . . 86

5.4 Simulation Studies . . . . . . . . . . . . . . . . 88

5.4 .1 Physiologic parameter change . . . . . . . . . . . . 88

5.4 .2 Robustness to Noise . . . . . . . . . . . . . . . . 97

5.5 How Do the Discriminant scores behave over time? . . . . . . . . . . . . . 99

6.0 A RULE-BASED CONTROLLER FOR ROTARY BLOOD PUMPS . 103

6.1 Why a Rule-based controller ? . . . . . . . . . . . . . . . . . 104

6.2 Rule-based controller design . . . . . . . . . . . . . . . . 106

6.2.1 Rule base design . . . . . . . . . . . . . . . . . . . . . . 107

6.2 .2 Effects of Asymmetry in output sets . . . . . . . . . . . . . 111

6.2 .3 Final design . . . . . . . . . . . . . . . . . . . . . 113

6.2.3.1 Membership sets . . . . . . . . . . . . 113

6.2.3.2 Rule base . . . . . . . . . . . . . . . . . . . . . . 114

6.3 Simulation Studies . . . . . . . . . . . . . . . . . . 117

6.3.1 Initial pump speed . . . . . . . . . . . . . . . . . 117

6.3.2 Physiologic parameter change . . . . . . . . . . . . . . . . 120

6.3.2.1 Tuning the controller to very sick patients . . . . . . . . . . . 127

6.3.3 Hemodynamic Analysis . . . . . . . . . . . . . . . . . . . . . 129

6.3.4 Robustness to Noise . . . . . . . . . . . . . . . . . . . . . . . . . 132

7.0 DISCUSSION AND CONCLUSION . . . . . . . . . . . . 136

7.1 Cardiovascular-pump model . . . . . . . . . . . . . . . . . 137

7.2 Suction detection system . . . . . . . . . . . . . . . . . . 137

7.2.1 Meaning of the prior probabilities . . . . . . . . . . 138

7.2.2 Comparison with current technology . . . . . . . . . . . 139

7.3 Rule-based controller . . . . . . . . . . . . . . . . . . . . . 142

7.3.1 Tuning of Controller . . . . . . . . . . . . . . . . . 142 
7.3 .2 Emergency mode . . . . . . . . . . . . . . . . . . . . . . . 143

7.4 Contributions and Future Work . . . . . . . . . . . . . . . . . 144

7.5 Conclusion . . . . . . . . . . . . . . . . . . . 145

APPENDIX A. STATE EQUATIONS FOR THE CARDIOVASCULAR

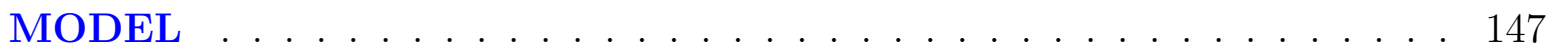

APPENDIX B. STATE EQUATIONS FOR THE CARDIOVASCULARPUMP MODEL . . . . . . . . . . . . . . . . . . . . . . . 149

APPENDIX C. CONFUSION MATRICES - SVR TESTS . . . . . . . . . 152

APPENDIX D. FAM BANKS OF CONTROL SURFACES . . . . . . . . . . 154

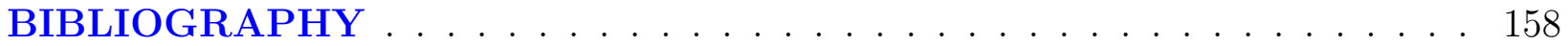




\section{LIST OF TABLES}

1 State Variables of the cardiovascular model . . . . . . . . . . . . . . . 27

2 Model Parameters . . . . . . . . . . . . . . . . . . . . . . . . . . 28

3 Phases of the cardiac cycle . . . . . . . . . . . . . . . 30

4 State Variables of the cardiovascular-pump model . . . . . . . . . . . . . 37

5 Example of a Confusion Matrix for a two-group classifier . . . . . . . . . . . . 69

6 Expected number classified by chance . . . . . . . . . . . . . . 70

7 Data Statistics . . . . . . . . . . . . . . . . . . . . 75

8 MANOVA Results for groups . . . . . . . . . . . . . . . . . . 77

9 DDA result for the two-group classification problem . . . . . . . . . 80

10 Confusion Matrix for the classifier ${ }^{a} \ldots \ldots \ldots$. . . . . . . . 81

11 Confusion Matrix for test set ${ }^{a} \ldots \ldots \ldots \ldots$. . . . . . . 82

12 DDA result for the three-group classification problem . . . . . . . . . . 84

13 Confusion Matrix for training set . . . . . . . . . . . . . . . 86

14 Confusion Matrix for test $\operatorname{set}^{a} \ldots \ldots \ldots \ldots$. . . . . . . 87

15 Parameter changes to evaluate the suction detector f . . . . . . . . . 90

16 Controller Rule-base with five output sets . . . . . . . . . . . . . . . 115

17 Parameter changes to evaluate the suction detector f . . . . . . . . 121

18 Pump states . . . . . . . . . . . . . . . . . . . . . . . . . . 140

19 Comparison between the three suction detectors . . . . . . . . . . . . 141

20 Controller Rule-base for three output sets . . . . . . . . . . . . . . . 155

21 Controller Rule-base for five output sets . . . . . . . . . . . . . . . 156

22 Controller Rule-base for seven output sets . . . . . . . . . . . . . . . 157 


\section{LIST OF FIGURES}

1 Anatomy of the human heart modified from A. Guyton and J. Hall [15] . . 6

2 Block diagram representation of the human circulation . . . . . . . . 7

3 Cardiac cycle indicating systolic and diastolic phases modified from A. Guyton and J. Hall $[15] \ldots \ldots$. . . . . . . . . . . . . . . . . . . . 8

4 Elastance function for a $75 \mathrm{bpm}$ heart rate . . . . . . . . . . . . 9

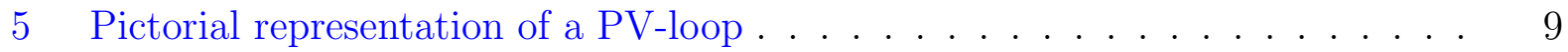

6 Resistance effects in vessels and their electrical equivalent . . . . . . . . . . 11

7 Compliance effect in vessels and its electrical equivalent . . . . . . . . . . . 11

8 Inertance property of a vessel and its electrical equivalent . . . . . . . . . 12

9 Ventricular models proposed by (a) McInnis and (b) Avanzolini . . . . . . . . 13

10 Windkessel models of the afterload system . . . . . . . . . . . . . . . . 14

11 Rule based controller . . . . . . . . . . . . . . . . . . . . . . . 23

12 An illustration of the height defuzzification method . . . . . . . . . . . . . 24

13 Cardiovascular Model . . . . . . . . . . . . . . . . . . . . . . . . . . . . . 26

14 Normalized Elastance (left panel), Elastance $E(t)$ (right panel) $\ldots . . . .29$

15 All integrator block diagram for the cardiovascular model . . . . . . . . . . . 32

16 Simulated hemodynamic waveforms for a normal subject . . . . . . . . . . 33

17 PV-loops used for model validation . . . . . . . . . . . . . . . . 34

18 Input impedance of the afterload system . . . . . . . . . . . . . . . . 34

19 Input impedance spectrum . . . . . . . . . . . . . . . . . . . 35

20 Curve fitting using human clinical data . . . . . . . . . . . . . . 35

21 Cardiovascular-pump Model . . . . . . . . . . . . . . . . . . . 36 
22 All integrator block diagram for the cardiovascular-pump model . . . . . . . . 39

23 Cardiac Output for step speed test . . . . . . . . . . . . . . . 41

24 Pump flow for step speed test; dotted line: $E_{\max }=1.0$; solid line: $E_{\max }=0.541$

25 Pressures for step speed test; dotted line: $E_{\max }=1.0$; solid line: $E_{\max }=0.5 \quad 42$

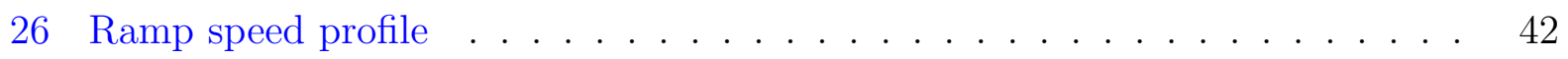

27 Hemodynamic variables for ramp test . . . . . . . . . . . . . 43

28 Simulation Results of the Cardiovascular-model. (a) Pump Speed, (b) Pump Flow from in-vivo test, (c) Pump Inlet Pressure from in-vivo test, (d) Pump Flow from model, and (e) Pump Inlet Pressure from model. . . . . . . . . . . 44

29 Pictorial representation of the suction phenomena: (a) normal, (b) suction . . 45

30 Schematic of the suction detection system . . . . . . . . . . . . . 46

31 Illustration and definition of $R_{I N} \ldots \ldots \ldots$. . . . . . . . . . 47

32 Experimental Nimbus data . . . . . . . . . . . . . . . . . 49

33 Hemodynamic variables for the three pumping states . . . . . . . . . . 49

34 Inflow resistance $R_{I N} \ldots \ldots \ldots \ldots$. . . . . . . . . . . . 50

35 Mean diastolic inflow resistance . . . . . . . . . . . . . . . . 50

36 Spectrum of pump flow . . . . . . . . . . . . . . . . . 52

37 Example of how PSD energy changes as suction occurs; (a) Pump Inlet Pressure, (b) Pump Flow. The bottom panels are expanded segments of Pump Flow with respective spectrums. . . . . . . . . . . . . 54

38 Simulation result of $S I_{1}$ and $S I_{2}$ to in-vivo data; (a) Pump Flow, (b) $S I_{1}$ and $S I_{2}$ as functions of time . . . . . . . . . . . . . . . . 55

39 Example of Maximum and minimum envelopes of (a) $q_{P}(t),(\mathrm{b}) d Q_{P} / d t \ldots 56$

40 Simulation results of time indices to in-vivo data; (a) Pump Flow, (b) $S I_{3}$, (c) $S I_{4}$ and $S I_{5},\left(\right.$ d) $S I_{6}$ and $S I_{7} \ldots \ldots \ldots \ldots \ldots$

41 Spectogram results and Instantaneous frequency of Pump Flow for 3 time windows. A) No Suction, B) Moderate Suction, and C) Severe Suction. In each case, panels from the top are PF, Spectrogram of PF, and the Instantaneous mean frequency of $\mathrm{PF},\langle\omega\rangle_{t}^{s p}$ respectively. . . . . . . . . . . . . 
42 Fisher's idea for discriminant analysis. In (a), $X_{1}$ is used to discriminate between groups 1 and 2 ; in (b), a linear combination of $X_{1}$ and $X_{2}$ does a better result. . . . . . . . . . . . . . . . . . 62

43 Within- and between-group variance estimates . . . . . . . . . . . 64

44 Fisher's idea for three-group discriminant analysis . . . . . . . . . . . . 66

45 Detector design steps . . . . . . . . . . . . . . . . . . . . . 74

46 Box plots of the features per group . . . . . . . . . . . . . . . 76

47 Hit-rate versus number of samples per group . . . . . . . . . . . . . 78

48 Histograms of discriminant scores for training set . . . . . . . . . . . . 81

49 Histograms of discriminant scores for test set . . . . . . . . . . . . . 83

50 Plot of training data in discriminant function space . . . . . . . . . . 85

51 Plot of test data in discriminant function space . . . . . . . . . . . . 87

52 Block diagram of cardiovascular-pump model and suction detection . . . . . 88

53 Test result of parameter changes for the healthy heart $\left(E_{\max }=2.0 \mathrm{mmHg} / \mathrm{ml}\right)$. Panels from the top are PS (Pump Speed), PF (Pump Flow), Discriminant Scores and DA model and Expert classification. . . . . . . . . . . . . . . 91

54 Test result of parameter changes for the healthy heart $\left(E_{\max }=2.0 \mathrm{mmHg} / \mathrm{ml}\right)$. Panels from the top are PS (Pump Speed), PF (Pump Flow), Discriminant Scores and DA model and Expert classification. . . . . . . . . . . . . .

55 Test result of parameter changes for the sick heart $\left(E_{\max }=1.0 \mathrm{mmHg} / \mathrm{ml}\right)$. Panels from the top are PS (Pump Speed), PF (Pump Flow), Discriminant Scores and DA model and Expert classification. . . . . . . . . . . . .

56 Test result of parameter changes for the sick heart $\left(E_{\max }=1.0 \mathrm{mmHg} / \mathrm{ml}\right)$. Panels from the top are PS (Pump Speed), PF (Pump Flow), Discriminant Scores and DA model and Expert classification. . . . . . . . . . . .

57 Test result of parameter changes for the very sick heart $\left(E_{\max }=0.6 \mathrm{mmHg} / \mathrm{ml}\right)$. Panels from the top are PS (Pump Speed), PF (Pump Flow), Discriminant Scores and DA model and Expert classification. . . . . . . . . . . . 
58 Test result of parameter changes for the very sick heart $\left(E_{\max }=0.6 \mathrm{mmHg} / \mathrm{ml}\right)$. Panels from the top are PS (Pump Speed), PF (Pump Flow), Discriminant Scores and DA model and Expert classification. . . . . . . . . . . . 96

59 Detector hit-rate as a function of contractility state . . . . . . . . . . . 97

60 Comparison between raw and noisy simulated pump flow . . . . . . . . . 98

61 Detector hit-rate as a function of SNR . . . . . . . . . . . . . . . 99

62 DA results for data File \# 20. (a) Pump Speed, (b) Pump Flow, (c) Detector output and Expert decision, and (d) Discriminant Scores. Capital letters indicate pump state transitions: $\mathrm{A}, \mathrm{NS} \rightarrow \mathrm{MS} ; \mathrm{B}, \mathrm{D}, \mathrm{F}, \mathrm{H}$ and $\mathrm{J}: \mathrm{MS} \rightarrow \mathrm{SS}$; C, $\mathrm{E}, \mathrm{G}, \mathrm{I}: \mathrm{SS} \rightarrow \mathrm{MS} \ldots \ldots \ldots \ldots$

63 Pump status point in the discriminant score plan. The dark crosses represent the group means. The small numbers at the side of the points represent the time information. Dashed lines connect consecutive time windows. Solid lines represent transitions in pump state, and capital letters indicate crossing boundaries according to changes shown in Figure 62 . . . . . . . . . . . . . . 102

64 Pump speed update . . . . . . . . . . . . . . . . 106

65 Input-output sets examined to derive the rule base. Top panels are input sets definitions and bottom panels are output sets. . . . . . . . . . . . . 108

66 Control surfaces . . . . . . . . . . . . . . . . . . . . 109

67 Simulation results of pump speed for control surfaces. Top panel shows low initial pump speed and bottom panels shows high pump speed. . . . . . . . . 110

68 Operating point on control surfaces. Only the last five points are shown with time information. The last five points are coincidents in (b) . . . . . . . . . . 110

69 Output Membership sets . . . . . . . . . . . . . . . . 111

70 Control surfaces for assymetric sets (a) controller A, (b) controller B . . . . . 112

71 Pump speeds results for asymmetric sets: (a) $\omega_{0}=8 \mathrm{krpm}$, (b) $\omega_{0}=9 \mathrm{krpm}$, (c) $\omega_{0}=13 \mathrm{krpm}$ and $(\mathrm{d}) \omega_{0}=14 \mathrm{krpm} . \ldots \ldots$. . . . . . . . . . 113

72 Controller Membership Sets. (a) Input variables; (b) Output Variables. (NB: Negative Big, NS: Negative Small, ZE: Zero, PS: Positive Small, PB: Positive Big) 114

73 Controller transfer characteristic . . . . . . . . . . . . . 116 
74 Controller Simulation Results . . . . . . . . . . . . . . . . . . . . . . . . 119

75 Aortic and Left Ventricular Pressures . . . . . . . . . . . . . . . 120

76 Block diagram of feedback control . . . . . . . . . . . . . . . . . . . 122

77 Controller results of Preload changes for the healthy heart $\left(E_{\max }=2.0\right) \quad \ldots 124$

78 Controller result of Preload changes for the sick heart $\left(E_{\max }=1.0\right) \ldots 125$

79 Controller result of Preload changes for the very sick heart $\left(E_{\max }=0.6\right)$. Note that controller fails for these test conditions. . . . . . . . . . . . 126

80 Controller result of Preload changes for the very sick heart $\left(E_{\max }=0.6\right)$ with modified membership output sets. . . . . . . . . . . . . . . . . 128

81 Delay on pump speed with modified membership output sets . . . . . . . . 129

82 Hemodynamic variable results for the first scenario (baseline $\rightarrow$ strenuous exercise $\ldots \ldots \ldots \ldots \ldots \ldots \ldots \ldots$

83 Hemodynamic variable results for the second scenario (baseline $\rightarrow$ hypertension) 132

84 RMSE as a function of SNR . . . . . . . . . . . . . . . . . . . . . 134

85 Example of deteriorated performance at $\mathrm{SNR}=10 \mathrm{~dB}$. Signals from the top are pump flow, pump speed, discriminant scores and the model classification result. Note that the suction event at $\mathrm{t}=250$ s was missed by the suction detector, causing the controller to erroneously increase pump speed. . . . . . . . . . . . 135 


\section{PREFACE}

This dissertation is the outcome of four years of research work I have performed as a member of the Physiologic Control team at the University of Pittsburgh. As such, it is not really a sole author work, but has contributions from those who have collaborated for my development as a researcher. In special, professors Robert Boston and James Antaki. Their guidance and encouragement have provided me with knowledge and expertise that will last a lifetime. A very special thanks to Professor M. Simaan for his helpful discussion and comments that contribute to improve some parts of this work.

I also want to thank the others students in our team: Shaohui Chen, Douglas McConahy, Bronwyn Uber and Dorian Arnold for their support. My special gratitude to the Coldebella family, Brenno Coelho and Ismet Sahin whose words of encouragement helped me in many decisive moments.

I would like to express my gratitude to the Alcoa Foundation, the Department of Electrical and Computer Engineering of the University of Pittsburgh, the Universidade Federal do Maranhao (UFMA/Brazil), and the Fundacao de Amparo a Pesquisa e ao Desenvolvimento Cientifico e Tecnologico do Maranhao (FAPEMA/Brazil) for sponsoring my graduate studies.

I would like to dedicate this work to my family: my beloved wife Rosa, my daughter Amanda and my son Joao; to my parents and family in Brazil, whose dedication and love have brought me at this point. Finally, but not least, to God.

"The Lord is my shepherd, I shall not be in want."

Psaml 23

Antonio Ferreira,

Pittsburgh, June $6^{\text {th }} 2007$. 


\subsection{INTRODUCTION}

Cardiovascular Disease is a major health problem in the United States [1]. Standard care entails drug administration to ensure blood perfusion. When the pharmacological treatment fails, heart transplantation has become an accepted method to treat severe cases of the disease. However, the demand exceeds the supply of organ donors, and many patients die while waiting for a transplant.

As the patient's heart condition deteriorates, mechanical support may be the only option to assist the impaired heart. Ventricular assist devices (VADs) have traditionally been used as a "bridge for transplantation", and now are being implanted as "bridge to recovery". In the latter option, the VAD reduces the workload imposed on the heart, which then can recover its contractility power. In all situations it is desirable to provide the patient with a life style as "normal" as possible $[2,3]$.

VADs can be classified as either positive-displacement (pulsatile) or dynamic pumps, depending on how they generate blood flow. Pulsatile VADs mimic the natural heart flow, operating in a beat-like fashion. Dynamic VADs usually deliver continuous flow and do not necessarily create pulsatile pressures and output flows. Rotary VADs are smaller, have high efficiency and do not need valves like their pulsatile counterparts. On the other hand, rotary VADs pose a more demanding control problem [4].

Two constraints should be taken into account regarding the operating settings for the pump speed of rotary devices: first, the rotational speed cannot be too low to avoid regurgitation, i.e, the return of blood from the aorta to the left ventricle through the pump (backflow); second, the rotational speed cannot be too high to avoid suction, i.e, an event that occurs when the rotary pump tries to draw more blood than is available. Suction may cause ventricular collapse to occur, which can cause chest pain and cardiac tissue damage. 
In recent years, several approaches have been proposed to solve the suction detection problem. These approaches are based on empirical observation of certain variables. For example, the spectral energy content of signals, such as pump flow and pump current, change when suction occurs. When the VAD runs at speeds below the region of suction, the pump flow is periodic at the patient's heart rate. Most of the energy power spectrum is concentrated around the fundamental frequency of the signal. When suction occurs, energy shifts to higher harmonics in the signal. A Normalized Second Harmonic index (NSH), defined as the ratio of second harmonic to the first harmonic, reaches a minimum at the onset of ventricular collapse [5]. Similarly, a waveform deformation index (WDI) based on power spectral density (PSD) analysis of the pump current to detect the occurrence of regurgitation and suction was proposed in [6]. The WDI is defined as the ratio of the fundamental component of the PSD to the higher PSD components. However, this ratio is not a unimodal function of pump speed (PS) and additional information is needed to decide between regurgitation and suction.

Pulsatility in the pump flow signal can be used to define a pulsatility index [7]. Even though the pump is a continuous flow device, the impaired ventricle still has pulsatile behavior. As a consequence, hemodynamic signals such as aortic pressure, left ventricular pressure and pump flow exhibit varying degrees of pulsatility during LVAD $^{1}$ support. As pump speed increases and ventricular unloading occurs, the pulsatility of all these signal decreases and reaches a minimum as suction is approached. Based on this concept, a proportional-integral type fuzzy-logic controller was proposed by Choi el al [7].

Another heuristic approach to detect suction was introduced by Antaki et al [8, 9]. The "Diminishing Returns Index" (DRI) is defined as the derivative of the mean pump flow with respect to pump speed $\left(\frac{d Q}{d \omega}\right)$. The DRI is based on the fact that mean pump flow rate decreases as pump speed increases. When venous return is matched by the pump, the rate of change is approximately zero. Thus, an appropriate operating point for the pump lies in the region for which $\frac{d Q}{d \omega}$ is slightly positive.

Suction patterns vary considerably. Therefore, one index may respond more effectively to a certain pattern than others, and the combined response to multiple indices may identify a broader range of patterns than a detector based on only one index. Baloa [3, 10] proposed

\footnotetext{
${ }^{1}$ VADs are usually referred as LVADs because their common application is to support the left ventricle
} 
a data-fusion method to combine these indices. He presents a measure of uncertainty for each index as well as for the combined result.

Volkron et al. [11] present several indices to detect suction. These indices are based on a time-domain analysis of pump flow patterns from VAD-implanted patients. Using a window length of 5 seconds, patterns extracted from the pump flow wave form were compared against snapshots of pump flow previously stored and classified in a data base by human experts, in order to decide whether suction is present. The authors acknowledge the fact that the proposed method can easily increase exponentially the possible combinations in its optimization procedure, if applied to a multiple-beat analysis. This might be an issue for real time applications of the proposed method.

Recently, we described a suction detector based on frequency indices combined with a time-frequency index [12]. The frequency based indices can detect changes in the harmonic and subharmonic energy content of the pump flow signal that occur during a suction event. The time-frequency index can track variations in the standard deviation of the instantaneous frequency of that signal. These two pieces of information are then combined in a weighted decision system to generate a suction alarm.

This work was expanded by supplementing the frequency indices and the time-frequency index with time indices that can detect changes in pump flow pulsatility based on a beat-tobeat analysis of the pump flow and first derivative of pump flow [13]. These indices are then combined using Discriminant Analysis (DA) in order to classify the pump status in one of the following three categories: No Suction (NS), Moderate Suction (MS) or Severe Suction (SS).

Using the discriminant scores as inputs, a rule-based controller was designed [14]. This controller can automatically adjust pump speed, providing cardiac output and pressure perfusion according to the patient's level of activity. This dissertation describes the design and simulation studies performed for both modules of the control system: the suction detection system and the rule-based controller.

This dissertation is organized as follows. Chapter 2 presents some concepts on cardiovascular physiology and analog models of the cardiovascular system. Chapter 3 reviews some definitions regarding fuzzy logic that are used in designing the rule-based controller. The 
cardiovascular-pump model used for simulation studies is developed in Chapter 4, and some simulation results are presented. Chapter 5 describes the discriminant-analysis-based suction detection system, and preliminary results of that system using in-vivo data are presented. Chapter 6 describes the rule-based controller design and presents simulation results of the control system (suction detector and controller). Final thoughts and contributions of this work are discussed in Chapter 7. 


\subsection{CARDIOVASCULAR PHYSIOLOGY}

Some basic concepts regarding cardiovascular physiology are presented in this chapter. These concepts are important to understand the cardiovascular model used, as well as the control objectives for the rotary LVAD. The chapter is organized as follows. The heart muscle and the circulation are described. After that, the cardiac cycle and some properties of the vessels are presented. Finally, lumped parameter models of the vascular bed system are described.

\subsection{HEART AND CIRCULATION}

The heart is a single organ that can be thought of as two separate pumps, one on each side of the heart (left and right; see Figure 1). Each pump contains two chambers: atrium and ventricle. The heart functions as a volume displacement pump. Its main purpose is to propel blood from one section of the circulatory system to another. The left heart (atrium and ventricle) pumps blood into the systemic circulation, while the right heart maintains the pulmonary circulation. There are unidirectional valves connecting these chambers in each side of the heart, and also with the circulation. The mitral valve connects the left atrium (LA) to the left ventricle (LV). Likewise, the tricuspid valve connects the right atrium (RA) to the right ventricle (RV). The aortic valve links the LV and the aorta, and the pulmonary valve links the pulmonary artery to the RV.

Figure 2 illustrates the circulation process in the human body. Starting at the right ventricle, blood is pumped into the pulmonary circulation via the pulmonary artery. The pulmonary artery branches into the left and right halves of the lung. In the lungs, the blood in the capillaries binds oxygen and releases carbon dioxide. The oxygenated blood leaves 


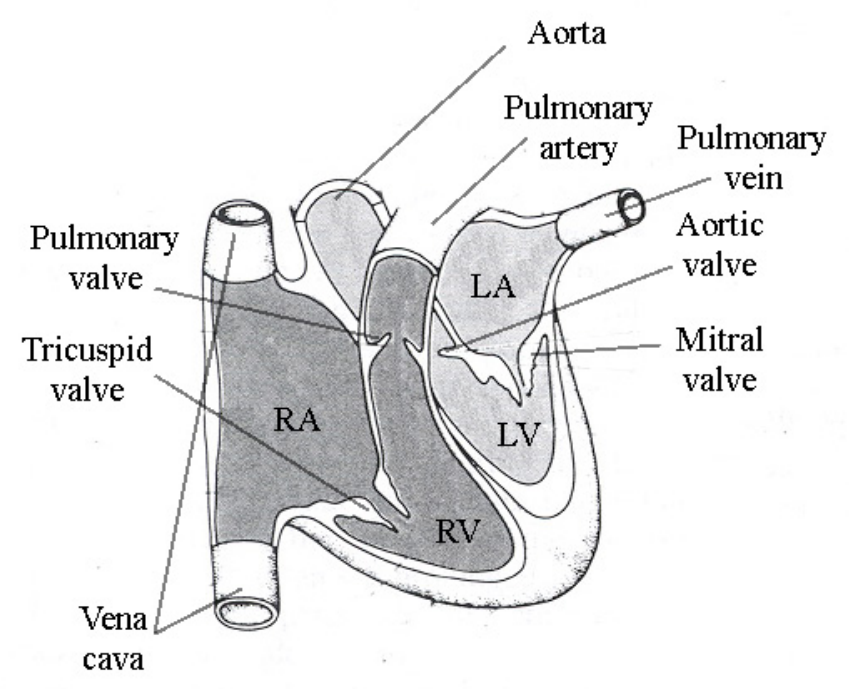

Figure 1: Anatomy of the human heart modified from A. Guyton and J. Hall [15]

the lungs and reaches the left atrium through the pulmonary vein. Blood in the left atrium is pumped into the left ventricle, which pumps it into the aorta. The aorta branches into large arteries, small arteries, and arterioles. These vessels then branch to form the systemic capillaries and it is at this level that the exchange of substances occurs between blood cells and organ cells.

Contiguous to the capillaries are the venules, which are the smallest vessels that transport the low-oxygen blood back to the heart. Blood then travels from the venules to the systemic veins, and eventually the vena cavae, which is connected to the right atrium of the heart, closing the loop. 


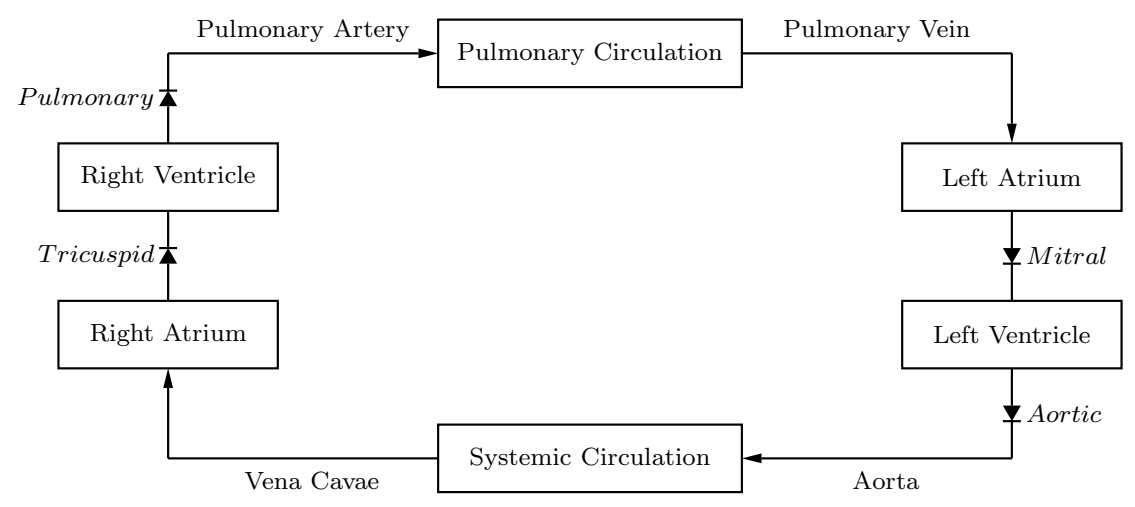

Figure 2: Block diagram representation of the human circulation

\subsection{THE CARDIAC CYCLE}

The cardiac cycle is the period of time between two consecutive heart beats. It consists of two phases: a period during which the heart is contracting - systole, and a period during which the heart is relaxing - diastole. Under normal conditions, the diastolic interval is longer than the systolic. However, when the heart rate increases, the diastolic period is shorter, which implies that the heart fills less.

Figure 3 shows the cardiac cycle from the stand point of the left ventricle. Systole starts at point 1 in Figure 3 with isovolumic contraction. In this phase, the ventricle is a completely sealed chamber. The ventricle starts to contract and the pressure developed closes the mitral valve. However, the left ventricular pressure (LVP) developed during this phase is not sufficient to overcome aortic pressure (AoP). Consequently the aortic valve remains closed and no blood leaves the ventricle. Because there is no change in the volume of blood in the ventricle, this is called an isovolumic phase.

At point 2, left ventricular pressure exceeds aortic pressure and the aortic valve opens, ejecting blood into the aorta. The diastolic interval starts at point 3 , with isovolumic relaxation. LVP drops and the ventricular muscle relaxes with constant volume. When LVP decreases below left atrial pressure (LAP), the mitral valve opens, allowing blood into the ventricle, and a new cycle starts at point 4 . 


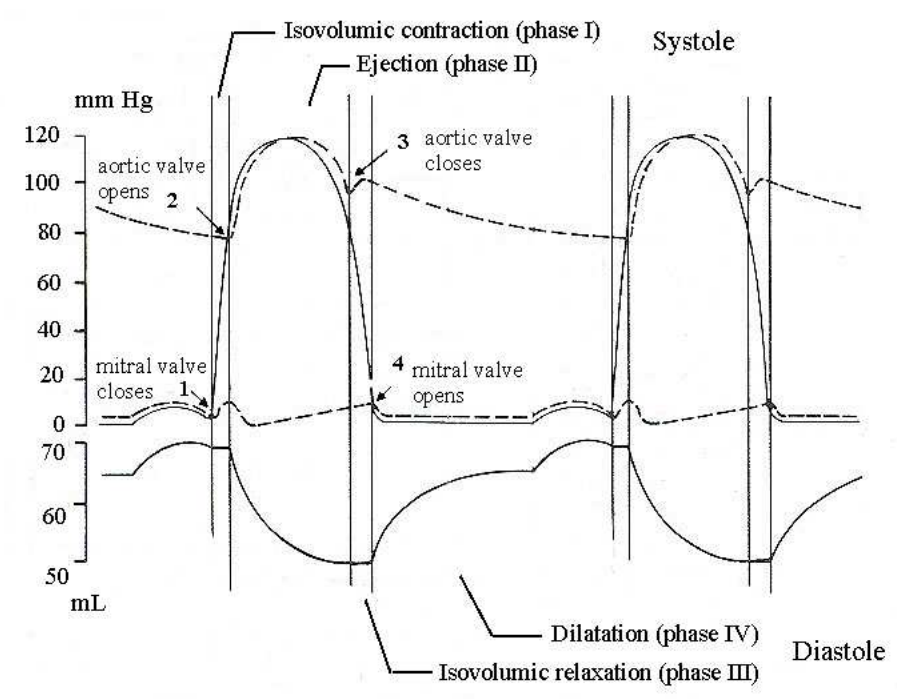

Figure 3: Cardiac cycle indicating systolic and diastolic phases modified from A. Guyton and J. Hall [15]

\subsection{THE PRESSURE-VOLUME RELATIONSHIP}

The time-varying elastance theory was introduced by Suga and Sagawa [16, 17]. Based on experiments with canine hearts, they found that the normalized elastance has the same shape for all loading conditions, contractile states, and heart rates. Moreover, the general curve is characteristic for each species. These features make the time-varying elastance theory suitable for use in models where different loading conditions will be simulated. The elastance is associated with the contractility of the heart and is defined as the ratio of left ventricular pressure LVP to left ventricular volume LVV, i.e.

$$
E(t)=\frac{L V P(t)}{L V V(t)-V_{0}}
$$

where $V_{0}$ is the volume at zero pressure. Figure 4 shows the elastance function for a heart rate of 75 beats per minute (bpm). The heart rate defines the duration of the cardiac cycle, i.e. the period of the elastance function. 


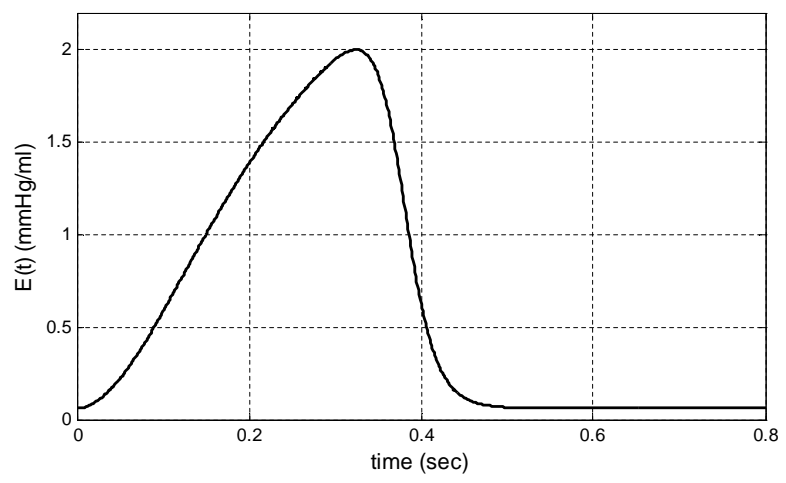

Figure 4: Elastance function for a $75 \mathrm{bpm}$ heart rate

Figure 5 shows a pressure-volume diagram (also known as PV loop). The dashed lines in Figure 5 represent the end-diastolic and the end-systolic pressure volume relations (EDPVR and ESPVR, respectively). The slope of a line connecting $V_{0}$ to a given point in that diagram determines the elastance value for that particular instant in time. . As time proceeds, the points evolve in a counterclockwise orientation in the loop.

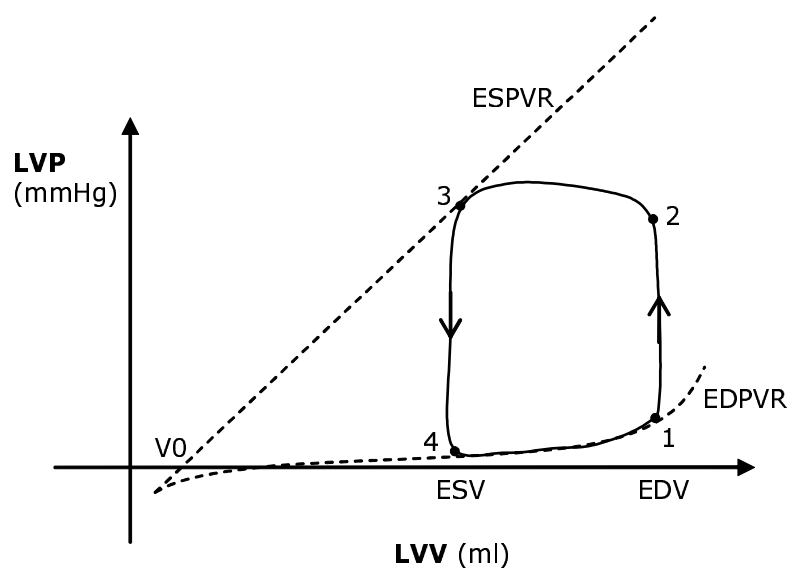

Figure 5: Pictorial representation of a PV-loop

The numbers shown in the PV loop in Figure 5 correspond to time points shown in Figure 1. Thus, number 1 represents the beginning of the isovolumic contraction phase, 2 coincides with ejection, 3 corresponds to isovolumic relaxation, and 4 to the filling phase.

Several important measurements can be derived from the PV loop, such as the maximum and minimum volume values in the cardiac cycle, i.e. the end-diastolic and end-systolic vol- 
umes (EDV and ESV) respectively. Their difference (EDV - ESV) represents the amount of blood ejected during the cardiac cycle, the stroke volume (SV). Another important measurement is stroke work (SW), the amount of energy transferred from the heart to the blood. i.e, the amount of energy required to eject the blood. SW is defined as the area within the PV loop.

\subsection{PROPERTIES OF VESSELS}

Blood vessels are characterized by several properties due to their material constitution, cross sectional area and length. Usually, the research goals dictate the complexity of a model. The properties considered here are fluid resistance, compliance and inertance. Since lumped linear parameters are used in this research, simple electrical analog elements are used to represent those properties. In Chapter 4, the analog elements presented here will be used to construct the cardiovascular-pump model. Therefore, our main interest is to describe such properties in order to model the vessels' behavior in a lumped-parameter model of the cardiovascular system.

Resistance in vessels is a phenomenon due to the frictional forces that oppose motion in a fluid. The resistance of a vessel varies with the viscosity of the blood, the length of the vessel, and its diameter. Resistance is defined by the ratio of the pressure drop along the longitudinal axis of the vessel to the flow [18], i.e,

$$
R=\frac{P(t)}{Q(t)}
$$

where $P(t)$ is the pressure drop along the vessel (measured in $m m H g$ ), $Q(t)$ is the flow through the vessel (measured in $m l / s$ ), and $R$ is hydraulic resistance with units of $\frac{m m H g \times s}{m l}$. An electrical equivalent model is the resistance element shown in Figure 6 where the voltage drop represents the pressure drop of the vessel and current represents the flow.

Compliance is the property of the vessel to expand and contract in response to internal pressure [3]. A distensible tube constructed of a compliant material that can expand and 

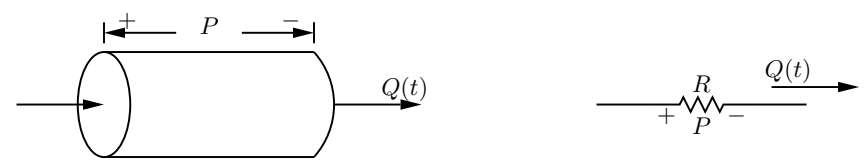

Figure 6: Resistance effects in vessels and their electrical equivalent

contract is shown in Figure 7. Let $P$ be the pressure difference between $p_{1}$ and $p_{2}$ then the relationship between volume, $V$, and the pressure difference, $P$, is

$$
V=C P
$$

where $C$ is the compliance of the tube and is dependent on the physical properties of the tube. The unit for compliance is $\frac{m l}{m m H g}$. Differentiating both sides of 2.2 and substituting flow, $Q$, for the derivative of volume results in:

$$
Q(t)=C \frac{d P}{d t}
$$

where $Q(t)$ is flow , $C$ is the compliance value, and $\frac{d P}{d t}$ is the rate of pressure change. An equivalent electric element for this equation is the capacitor, where the voltage represents pressure and the current represents flow. Figure 7 shows the compliance effect in a vessel and its electrical model.
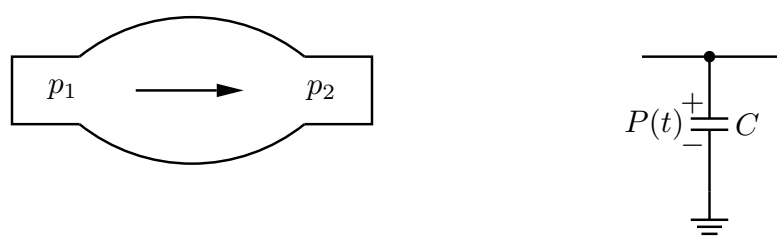

Figure 7: Compliance effect in vessels and its electrical equivalent

Fluid inertia models the effect of mass of fluids. Applying Newton's first law to moving fluids yields an equation in which the pressure drop along a vessel depends on dynamic changes of the flow and the mass characteristic of the fluid known as the inertia. The equation that governs such behavior is given by

$$
P(t)=L \frac{d Q}{d t}
$$


where $P$ is the pressure drop across the vessel, $L$ is the fluid inertia, and $\frac{d Q}{d t}$ is the dynamic change in flow. The last expression shows that hydraulic inertance is analogous to electrical inductance, with units of $\frac{m m H g \times s^{2}}{m l}$. In Figure 8, the voltage drop across the element is shown as $P, L$ is the inductance of the element and $Q(t)$ represents the current that flows through the element.

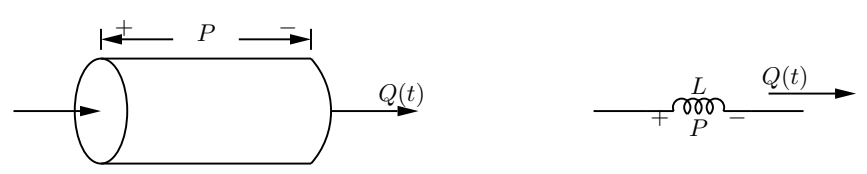

Figure 8: Inertance property of a vessel and its electrical equivalent

\subsection{MODELS OF THE CARDIOVASCULAR SYSTEM}

Mathematical models of the cardiovascular system can be classified as distributed and lumped parameter models. In this research, only lumped parameter models are considered. One advantage in representing the cardiovascular model as a lumped circuit is that Kirchoff's laws for node currents and loop voltages can be applied. In doing so, we can relate current with flow and voltage with pressure. Moreover, components like resistors, capacitors and inductors can be associated with hydraulic resistance, compliance and inertance, respectively.

In this section, research on modeling of the cardiovascular system is reviewed. First, some models of the left ventricle are described, and then afterload (windkessel) models are presented.

\subsubsection{Ventricular models}

The elastance-based ventricle model due to Suga et al. is still one of the most widely accepted for simulation and experimental purposes. Among those researches who used similar models is McInnis et al. [19] who model the ventricle by using the elastance approach, resistance and compliance properties. They approximated the ventricle as a linear system for the 
different phases of the cardiac cycle (systole and diastole), and developed control strategies for pulsatile air-driven assist devices. Figure 9(a) shows McInnis' model.

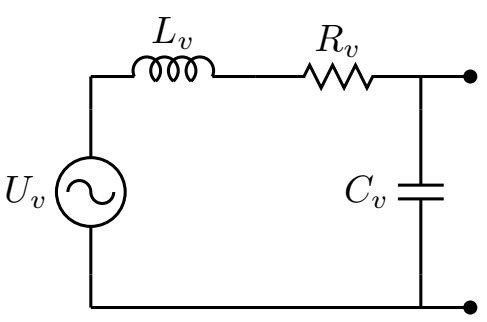

(a)

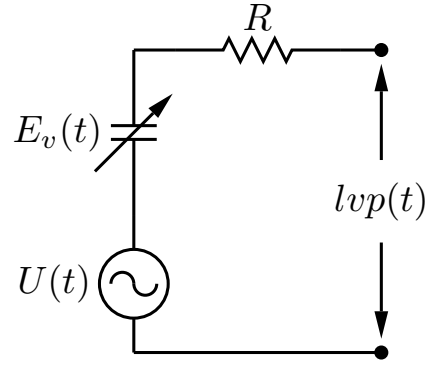

(b)

Figure 9: Ventricular models proposed by (a) McInnis and (b) Avanzolini

A similar modeling paradigm was used by Avanzolini et al. Figure 9(b) shows the electric circuit model of the ventricular muscle. The ventricular dynamics are described by

$$
\operatorname{lvp}(t)=\left\{\begin{array}{lr}
U(t)+E(t)\left(V(t)-V_{o}\right)+R \dot{V}(t) & \text { (during systole) } \\
E(t)\left(V(t)-V_{o}\right) & \text { (during diastole) }
\end{array}\right.
$$

where $\operatorname{lvp}(t)$ is the ventricular pressure, $V(t)$ is the ventricular volume, $V_{o}$ is the intercept of the ESPVR with the volume axis. $U(t)=U_{o} a(t)$ is the isovolumic pressure, when $a(t)=$ $\left(1-\cos \left(2 \pi t / t_{s}\right)\right) / 2$, during systole and $a(t)=0$ during diastole. $E(t)$ is the elastance function, defined as $E(t)=E_{d}+E_{s} a(t)$, and $R$ is myocardial resistance. This model produced pressure and flow waveforms similar to physiological waveforms, and it has been used in modeling of the complete circulatory system $[20,21]$.

\subsubsection{Afterload Models}

The afterload of the left ventricle can be defined as the load the heart has to pump against. Frank [22] introduced the first afterload lumped model shown in Figure 10(a). Frank's model consists of a compliance in parallel with a resistor, known as "windkessel".

A third element was added to the previous model by Westerhof [23], representing the resistance at the proximal aorta, $R_{c}$ - characteristic resistance. (see Figure 10(b)). Westerhof's model, also known as the three element windkessel model, has been extensively used because of the physiological meaning associated with its components. 


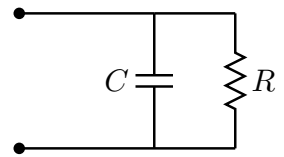

(a)

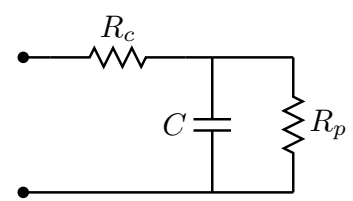

(b)

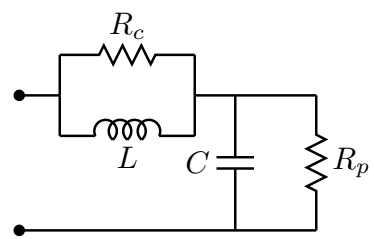

(c)

Figure 10: Windkessel models of the afterload system

Figure 10(c) presents one possible configuration of a four element windkessel model [24]. It presents an inductor $(L)$ either in series or parallel with the characteristic impedance $\left(R_{c}\right)$. This inductor represents the total inertance resulting from the fluid mass in the arterial system. The inertance is suitable for pulsatile models, where an acceleration of the mass fluid is usually required. In chapter 4 , these modeling concepts will be used to construct a complete cardiovascular model of the left heart. The next chapter presents a brief overview of fuzzy logic necessary to design the rule-based controller in chapter 6 . 


\subsection{FUZZY LOGIC CONTROLLER}

In this chapter, a brief overview of fuzzy logic is presented. This theoretical background $[25,27]$ is necessary to the application of a FLC to the problem of controlling a rotary assist device. Mamdani's work extended the use of Fuzzy Logic to control theory. For the control of non-linear, time-varying dynamic systems, conventional control theory might not be the only indicated approach. A Fuzzy Logic Controller (FLC) can be used either as an alternative to control such systems or in addition to conventional control techniques. This chapter is organized as follows: Fuzzy sets are defined and some of their properties and operations are presented. The concepts of fuzzy relation, composition and linguistic variable are subsequently presented. Finally, the design procedure of a FLC is shown.

\subsection{FUZZY SETS}

In classical set theory, let $A$ be a set defined on the universe $U$, then for any element $x$ of $A$, either $x \in A$ or $x \notin A$. There are two basic forms of defining a set: by enumerating its elements, or describing them by a property, say $P$. A third way is by a characteristic function, $\mu_{A}$.

Definition 1. $\mu_{A}: U \rightarrow\{0,1\}$ is a characteristic function of the set $A$ if and only if for all $x$

$$
\mu_{A}(x)= \begin{cases}1, & \text { when } x \in A \\ 0, & \text { when } x \notin A .\end{cases}
$$

In fuzzy set theory, the characteristic function is generalized to a membership function that assigns to every element $u \in U$ a value from the interval $[0,1]$. 
Definition 2. The membership function $\mu_{F}$ of a fuzzy set $F$ is a function $\mu_{F}: U \rightarrow[0,1]$. So, every element $u$ from $U$ has a membership degree $\mu_{F}(u) \in[0,1]$.

Finally, a fuzzy set can be formally defined as follows

Definition 3. A fuzzy set $F$ in a universe of discourse $U$ is completely determined by the set of tuples

$$
F=\left\{\left(u, \mu_{F}(u)\right) \mid u \in U\right\}
$$

We will refer to classical sets here as crisp sets. A consequence of definition 3 is that fuzzy sets are an extension of classical set theory since, for a certain universe, a membership function may act as an indicator function, mapping all elements to either 1 or 0 , as in the classical notion.

An alternative notation for the tuple $\left(u, \mu_{F}(u)\right)$ is $\mu_{F}(u) / u$, where / denotes tuple. Additionally, the "+" sign denotes an enumeration. Based on this, a countable or discrete universe $U$ allows a notation

$$
F=\sum_{u \in U} \mu_{F}(u) / u
$$

whereas when $U$ is uncountable or continuous, we have

$$
F=\int_{U} \mu_{F}(u) / u
$$

Thus, the $\int$ sign denotes an uncountable enumeration, not the usual integral definition.

\subsubsection{Properties of fuzzy sets}

The support of a fuzzy set $A$, denoted by $\mathcal{S}(\mathcal{A})$, is defined as

$$
\mathcal{S}(A)=\left\{u \in U \mid \mu_{A}(u)>0\right\}
$$

i.e., is the crisp set that contains all elements of $A$ with non-zero membership degree.

A fuzzy singleton is a fuzzy set whose support is a single point in $U$, that is

$$
\mu_{A}(x)= \begin{cases}1, & \text { if } x=x^{*} \\ 0, & \text { otherwise }\end{cases}
$$


The height of a fuzzy set $A$ on $U$, denoted by $\operatorname{hgt}(A)$, is defined as

$$
\operatorname{hgt}(A)=\sup _{u \in U} \mu_{A}(u)
$$

A fuzzy set $A$ is called normal if $\operatorname{hgt}(A)=1$.

A fuzzy set $A$ is convex if and only if

$$
\forall x, y \in U, \forall \lambda \in[0,1]: \mu_{A}(\lambda . x+(1-\lambda) . y) \geq \min \left(\mu_{A}(x), \mu_{A}(y)\right)
$$

This definition says that a fuzzy set is convex if and only if its $\alpha$-cuts ${ }^{1}$ are convex in the classical mathematical sense. In this research, only convex fuzzy sets are considered. There are several forms of defining membership functions for fuzzy sets. Usual examples of such functions for control applications are the $\Gamma, L$ and $\Delta$ functions defined as follows.

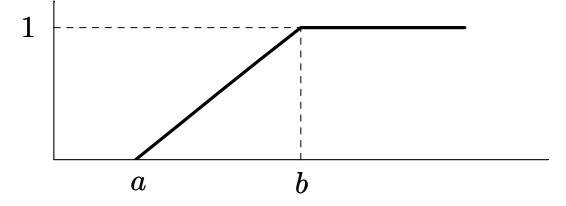

The function $\Gamma: U \rightarrow[0,1]$ is a function with parameters $a, b$ defined as

$$
\Gamma(x, a, b)= \begin{cases}0 & x<a, \\ (x-a) /(b-a) & a \leq x \leq b, \\ 1 & x>b .\end{cases}
$$

The function $L: U \rightarrow[0,1]$ is a function with parame-

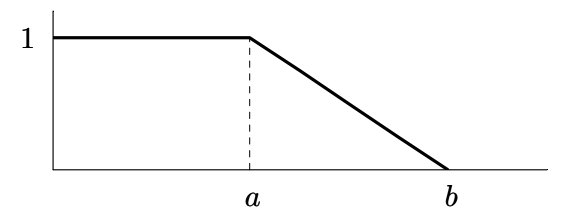
ters $a, b$ defined as

$$
L(x, a, b)= \begin{cases}1 & x<a, \\ (b-x) /(b-a) & a \leq x \leq b, \\ 0 & x>b .\end{cases}
$$

\footnotetext{
${ }^{1}$ An $\alpha$-cut of a fuzzy set $A$ is a crisp set defined as $A_{\alpha}=\left\{x \mid \mu_{A}(x) \leq \alpha\right\}$
} 
1

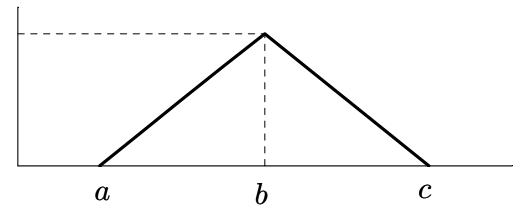

The function $\Lambda: U \rightarrow[0,1]$ is a function with parameters $a, b$ and $c$ defined as

$$
\Lambda(x, a, b, c)= \begin{cases}0 & x<a \text { or } x>c \\ (x-a) /(b-a) & a \leq x \leq b \\ (b-x) /(c-b) & b \leq x \leq c\end{cases}
$$

\subsubsection{Operations on Fuzzy Sets}

The classical set operations union, intersection and complement were extend to fuzzy sets by Zadeh [26] as follows. Let $A$ and $B$ be two fuzzy sets in the universe $U$, then

a) Union: $\forall x \in U: \mu_{A \cup B}=\max \left(\mu_{A}(x), \mu_{B}(x)\right)$

b) Intersection: $\forall x \in U: \mu_{A \cap B}=\min \left(\mu_{A}(x), \mu_{B}(x)\right)$

c) Complement: $\forall x \in U: \mu_{A^{\prime}}=1-\mu_{A}(x)$

More generally, triangular norms, s-norms and t-norms, can be used to define union and intersection operations, respectively:

$$
\begin{aligned}
& s\left[\mu_{A}(x), \mu_{B}(x)\right]=\mu_{A \cup B}(x) \\
& t\left[\mu_{A}(x), \mu_{B}(x)\right]=\mu_{A \cap B}(x)
\end{aligned}
$$

In the same fashion, the complement operation can be more generally defined as

$$
c\left[\mu_{A}(x)\right]=\mu_{\bar{A}}(x)
$$




\subsection{FUZZY RELATION}

In classical set theory, a relation is a set of ordered tuples, e.g., $\left(x_{1}, x_{2}, \cdots, x_{n}\right)$ is a $n$-tuple. In the same fashion, a fuzzy relation is a fuzzy set of tuples, i.e., each tuple has a membership degree in the interval $[0,1]$.

Definition 4. Let $U$ and $V$ be uncountable (continuous) universes, and $\mu_{R}: U \times V \rightarrow[0,1]$, then

$$
R=\int_{U \times V} \mu_{R}(u, v) /(u, v)
$$

is a binary relation on $U \times V$. If $U$ and $V$ are countable (discrete) universes, then

$$
R=\sum_{U \times V} \mu_{R}(u, v) /(u, v)
$$

\subsubsection{Fuzzy If-Then statements}

Let $x$ and $y$ be linguistic variables defined on the universes $U$ and $V$, respectively. Let $A \subseteq U$ and $B \subseteq V$ be fuzzy sets associated with $x$ and $y$, respectively. A fuzzy conditional or a fuzzy if-then production rule is expressed as

$$
\text { IF } x \text { is } A \text { THEN } y \text { is } B
$$

The meaning of this rule is represented as a fuzzy relation defined on $U \times V$. The construction of this fuzzy relation proceeds as follows:

a) the meaning of " $x$ is $A$ ", called the rule antecedent, is represented by a fuzzy set

$$
A=\int_{U} \mu_{A}(x) / x
$$

b) the meaning of " $y$ is $B$ ", called the rule consequent, is represented by a fuzzy set

$$
B=\int_{V} \mu_{B}(y) / y
$$

c) the meaning of the fuzzy conditional is then a fuzzy relation $\mu_{R}$ given as

$$
\forall x \in U, \forall y \in V: \mu_{R}(x, y)=\mu_{A}(x) \star \mu_{B}(y)
$$

where $\star$ can be any fuzzy implication operator. 
In control applications, the most common implication methods used are Mamdani's implications methods. Using the minimum implication method of Mamdani, the fuzzy relation $R$ is defined as $\mu_{R}(x, y)=\min \left(\mu_{A}(x), \mu_{B}(y)\right)$, and Mamdani product implication method is defined as $\mu_{R}(x, y)=\mu_{A}(x) \cdot \mu_{B}(y)$.

\subsubsection{Composition}

Let $A$ be a fuzzy set in $U$ and $R$ a fuzzy relation in $U \times V$. The fuzzy set $B$ in $V$ can be defined by the composition of $A$ and $R$ as

$$
\mu_{B}(y)=\sup _{x \in U}\left[\mu_{A}(x) \star \mu_{R}(x, y)\right]
$$

with $x \in U$ and $y \in V$. The symbol $\star$ is usually replaced with a $t$-norm (intersection) operator, and equation 3.1 is also known as sup-star composition. If the intersection is performed with the min operator and projection with maximum,

$$
\mu_{B}(y)=\max _{x} \min \left(\mu_{A}(x), \mu_{R}(x, y)\right)
$$

then the max - min composition is obtained. If intersection is performed with the product and projection with maximum,

$$
\mu_{B}(y)=\max _{x}\left(\mu_{A}(x) \cdot \mu_{R}(x, y)\right)
$$

which is called the max-dot or max-product composition. 


\subsubsection{Linguistic Variables}

According to Zadeh [28], a linguistic variable is a variable whose values are words or sentences in a natural language. For example, pump speed is a linguistic variable if its values are linguistic rather than numerical, i.e., high, low, very low. A framework usually associated with the notion of a linguistic variable is

$$
\langle\mathcal{X}, \mathcal{L} \mathcal{X}, \mathrm{X}\rangle
$$

where $\mathcal{X}$ denotes the name of the linguistic variable, e.g., pump flow, pump speed, etc. $\mathcal{L} \mathcal{X}$ is the set of linguistic values that $\mathcal{X}$ can take. A linguistic value is a word that usually describes a particular property of X. In the case of pump speed, we may have

$$
\mathcal{L} \mathcal{X}=\{\text { very high, high, low, very low }\}
$$

$\mathcal{L} \mathcal{X}$ is also known as the reference-set or term-set of $\mathcal{X}$. $\mathrm{X}$ is the actual physical domain of over which the linguistic variable $\mathcal{X}$ takes its quantitative (crisp) values. In the case of pump speed it can be the interval $[9,000,14,000] \mathrm{rpm}$.

\subsection{FUZZY CONTROL SYSTEMS}

Following Zadeh's seminal work [28], the theory of fuzzy sets and fuzzy logic has been applied to the control of complex ill-defined processes. Mamdani's fuzzy controller was one of the first proposed applications of fuzzy logic to process control [29]. Based on human knowledge obtained from experts and/or plant operators, this approach encodes such expertise as fuzzy rules of the form

$$
R_{i}: \text { IF }\left(x_{1} \text { is } A_{1}, x_{2} \text { is } A_{2}, \cdots \text {, and } x_{n} \text { is } A_{n}\right) \text { THEN } u \text { is } B
$$

where $\boldsymbol{x}=\left[x_{1}, x_{2}, \ldots, x_{n}\right]$ is the input vector, $A_{i}(i=1, \cdots, n)$ are the membership sets of $\boldsymbol{x}, u$ is the output and $B$ is its output membership set.

Mamdani's controller can be characterized as follows: 
a) the ranges of the input and output variables are either divided into a finite number of real intervals, each one of those being the support of a fuzzy set, or the variables can only take a finite number of real values;

b) there is a set of decision rules that specify a value of the controller output, for given discrete values or intervals of the controller inputs;

c) the rule or set of rules that is fired at a given time determines the actual value of the controller output at that time.

Takagi and Sugeno proposed a new type of fuzzy control system [27]. Their approach is based on a multiple model representation of the plant to be controlled. Each one of these models can be seen as a local model, being valid in a particular operating region. The operating regions are given by the conditional part, similar to the fuzzy rules of Mamdani's controller. However, the consequent part is an analytical expression describing the correspondent local model, rather than another fuzzy set as in Mamdani's approach. For instance,

$$
R_{i}: \quad I F\left(x_{1} \text { is } A_{1}, x_{2} \text { is } A_{2}, \cdots, \text { and } x_{n} \text { is } A_{n}\right) \text { THEN } y_{i}=f\left(x_{i}, p_{i}\right)
$$

where $\boldsymbol{x}=\left[x_{1}, x_{2}, \ldots, x_{n}\right]$ is the input vector, $A_{i}(i=1, \cdots, n)$ are the membership sets of $\boldsymbol{x}$, and $y_{i}$ and $p_{i}$ are the real output variable and parameters of the $i^{\text {th }}$ local model. In this research, only Mamdani's fuzzy control approach will be used.

\subsubsection{Design of a Fuzzy Logic Controller}

The design of a Fuzzy Logic Controller (FLC)) encompasses three main tasks: the design of the membership sets of the input and output variables, the rule base and the defuzzification method. Figure 11 illustrates the main components of a FLC. The FUZZIFICATION interface converts crisp input values $\boldsymbol{x}$ to input membership sets. Two pieces of information are provided by the fuzzification process: the linguistic values to which the input vector $\boldsymbol{x}$ belongs and their certainty levels as measured by the membership functions. 
The RULE BASE, also known as Fuzzy Associative Memory - FAM, comprises rules describing how the controller performs. These rules are usually written as IF-THEN rules of the form

$$
\text { IF }\left(x_{1} \text { is } A_{1}, x_{2} \text { is } A_{2}, \cdots \text {, and } x_{n} \text { is } A_{n}\right) \text { THEN } u \text { is } B
$$

where $\boldsymbol{x}=\left[x_{1}, x_{2}, \ldots, x_{n}\right]$ is the input vector, $A_{i}(i=1, \cdots, n)$ are the membership sets of $\boldsymbol{x}, u$ is the output and $B$ is its output membership set.

The INFERENCE ENGINE uses fuzzy reasoning to combine the fired IF-THEN rules in the rule base. It maps fuzzy input sets to fuzzy output sets. In this research, Mamdani's minimum implication method will be used to obtain the fuzzy output from the fuzzy implication rule. The fuzzy rule in equation (3.4) can be translated as

$$
\mu_{R_{i}}(x, y)=\mu_{R_{i}}\left(x_{1}, x_{2}, \ldots, x_{n}, y\right)
$$

where $R_{i}$ is a fuzzy relation (implication), $A=A_{1} \times A_{2} \times \ldots A_{n} \subseteq U, B \subseteq V$. For a given input set $A^{\prime}$ in $U$, the output fuzzy set $B^{\prime}$ in $V$ is expressed as

$$
\mu_{B_{i}^{\prime}}(y)=\sup _{x \in U}\left[\mu_{A^{\prime}}(x) \star \mu_{R_{i}}(x, y)\right]
$$

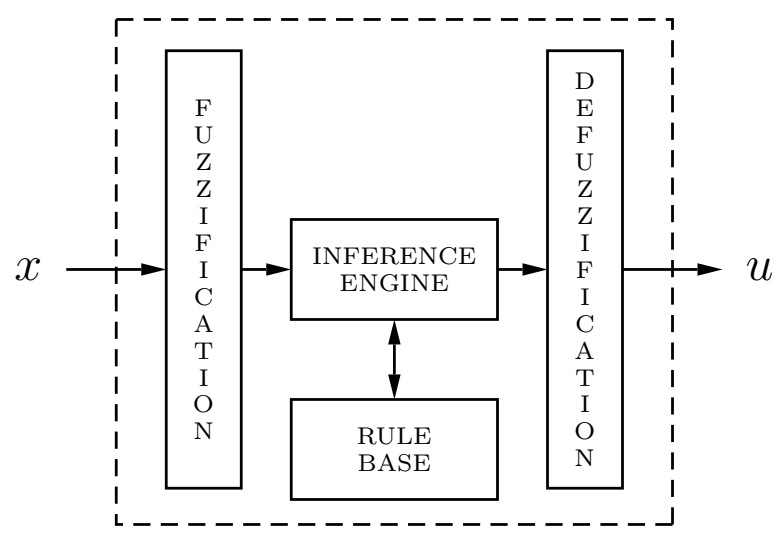

Figure 11: Rule based controller 
The DEFUZZIFICATION interface converts linguistic output sets to crisp values. In this research, the height method [26] is used because it is computationally simple. Figure 12 shows a graphical representation of the height defuzzification method. This method uses the individual center values, $c_{k}$, of the fired membership functions and builds the weighted (with respect to the height $f_{k}$ ) sum of these center values. The height defuzzification method in a system of $m$ rules is given as

$$
u=\frac{\sum_{k=1}^{m} c_{k} \cdot f_{k}}{\sum_{k=1}^{m} f_{k}}
$$

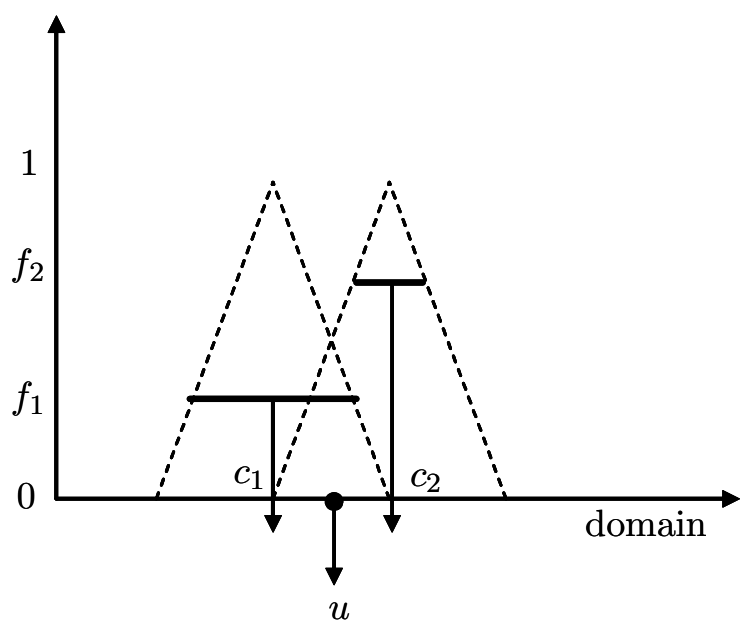

Figure 12: An illustration of the height defuzzification method

In [26] the height method is compared with others defuzzification methods, such as the center of area (also referred to as the center of gravity method) and the center of sums, presenting similar results. 


\subsection{THE CARDIOVASCULAR MODEL}

The human cardiovascular system is a time-varying distributed parameter nonlinear system. Nevertheless, simplified mathematical models of the cardiovascular system have been developed for many years for a variety of purposes. These include estimation and study of cardiovascular parameters difficult to measure in practical situations [21, 30, 31] and analysis and development of new medical products [32].

Recently, mathematical models of the human circulation have been developed also for studying its interaction with assist devices. Bai et al [33] presented a cardiovascular system model that includes a simulation of a cardiac assist device by external counterpulsation. It includes the left and right heart and the pulmonary circulation. De Lazzari et al [34] studied the interaction between a pneumatic left ventricle assist device (LVAD) and the cardiovascular system, by using energy variables, such as external work, oxygen consumption and cardiac mechanical efficiency for both fixed and variable heart rates. Computer models have been useful for simulating the interaction between the human cardiovascular system and assist devices, prior to in vitro and in vivo experiments [35, 36]. However, control strategies derived from such complex models have not been implemented yet due to many state variables that are not observable in practice. For the same reason, on-line identification of cardiovascular parameters remains a difficult task.

Breitenstein [18] proposed a univentricular model of the cardiovascular system, which was developed using a minimal number of parameters to make system identification as simple as possible. In this model, left ventricular compliance was modeled as a time-varying capacitor. Preload and pulmonary circulation were represented by a compliance, and a four element windkessel model was used as afterload. Yu [37] used that same model to develop an extended

Kalman filter estimator for the identification of systemic circulation model parameters during 
cardiac ejection and cardiac filling. Ferreira [38] has validated that model using human data. In addition, hemodynamic variables were simulated for different afterload conditions, and for the case of linearly increasing (ramp) pump speed, a test usually performed during in-vivo studies [39].

This chapter describes an extended version of that model. The addition of a capacitor representing aortic compliance made it possible to describe the dynamics in the model using only one set of differential equations. The chapter is organized as follows: the cardiovascular model is initially described and validated. Then, the cardiovascular-pump model is presented, and its open-loop response is analyzed for the cases of step and ramp input speed.

\subsection{THE CARDIOVASCULAR MODEL}

The cardiovascular model used in this research is shown in Figure 13. Preload and pulmonary circulation are represented by a single compliance, $C_{R}$, and afterload by a five-element windkessel model. Unlike the four element windkessel model shown in Chapter 2, the inductor is placed in series with the characteristic impedance. This was done to enhance the fitting capabilities of the model. Table 1 lists the state variables employed, and Table 2 provides the system parameters and their associated values [37].

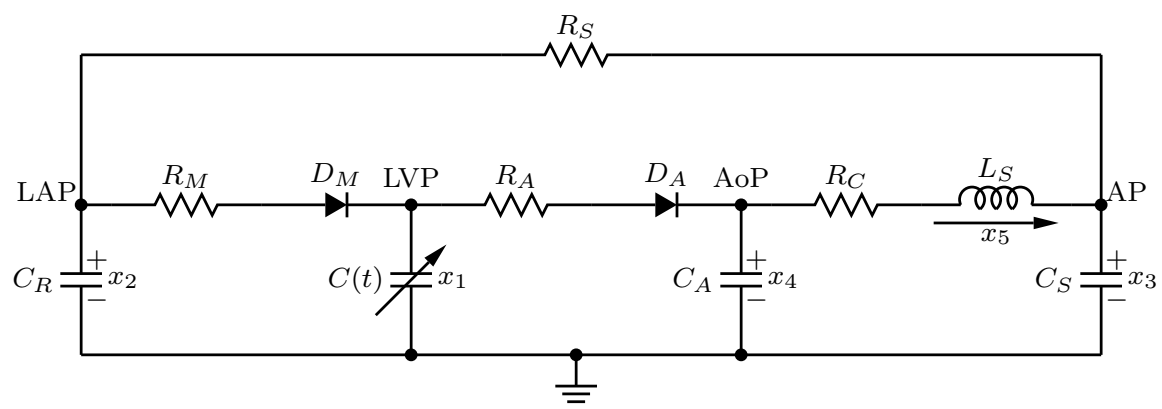

Figure 13: Cardiovascular Model 
Table 1: State Variables of the cardiovascular model

\begin{tabular}{c|l|l}
\hline Variables & Name & Physiological Meaning (unit) \\
\hline$x_{1}$ & LVP & Left Ventricular Pressure $(\mathrm{mmHg})$ \\
\hline$x_{2}$ & $L A P$ & Left Atrial Pressure $(\mathrm{mmHg})$ \\
\hline$x_{3}$ & $A P$ & Arterial Pressure $(\mathrm{mmHg})$ \\
\hline$x_{4}$ & $A o P$ & Aortic Pressure $(\mathrm{mmHg})$ \\
\hline$x_{5}$ & $Q_{T}$ & Total flow $(\mathrm{ml} / \mathrm{sec})$ \\
\hline
\end{tabular}

\subsubsection{Modeling the ventricle}

In our lumped parameter circuit, the left ventricle is described as a time-varying capacitor. One way to model its behavior is by means of the elastance function, which is the reciprocal of the compliance. The elastance determines the change in pressure for a given change in volume within a chamber and was defined following Suga and Sagawa as [16, 17]

$$
E(t)=\frac{L V P(t)}{L V V(t)-V_{0}}
$$

where $E(t)$ is the time varying elastance $(\mathrm{mmHg} / \mathrm{ml}), L V P(t)=x_{1}(t)$ is the left ventricular

pressure $(\mathrm{mmHg}), L V V(t)$ is the left ventricular volume $(\mathrm{ml})$ and $V_{0}$ is a reference volume $(\mathrm{ml})$, the theoretical volume in the ventricle at zero pressure.

Several mathematical approximations have been used to implement the elastance function. In this work, we use the so called "double hill" function [40], $E_{n}\left(t_{n}\right)$ (see eq. 4.2), because it has only one mathematical expression for both the systolic and the diastolic time intervals in the cardiac cycle.

$$
E_{n}\left(t_{n}\right)=1.55\left[\frac{\left(\frac{t_{n}}{0.7}\right)^{1.9}}{1+\left(\frac{t_{n}}{0.7}\right)^{1.9}}\right]\left[\frac{1}{1+\left(\frac{t_{n}}{1.17}\right)^{21.9}}\right]
$$

Additionally, the period of the double hill is directly related to the heart rate. The scaled elastance function is defined as

$$
E(t)=\left(E_{\text {max }}-E_{\text {min }}\right) \cdot E_{n}\left(t_{n}\right)+E_{\text {min }}
$$


Table 2: Model Parameters

\begin{tabular}{lcl}
\hline Parameter & Value & Physiological Meaning \\
\hline \hline \multicolumn{2}{l}{ Resistances } & (mmHg.s $/ \mathrm{ml})$ \\
$R_{S}$ & 1.00000 & Systemic Vascular Resistance \\
$R_{M}$ & 0.00500 & Mitral valve resistance \\
$R_{A}$ & 0.00100 & Aortic valve resistance \\
$R_{C}$ & 0.03980 & Characteristic resistance \\
\hline Compliances $(\mathrm{ml} / \mathrm{mmHg})$ \\
$C_{(t)} \quad$ variable & Left ventricular compliance \\
$C_{R}$ & 4.40 & Left Atrial compliance \\
$C_{S}$ & 1.33 & Systemic compliance \\
$C_{A}$ & 0.08 & Aortic compliance \\
\hline Inertance $\left(\mathrm{mmHg} . \mathrm{s}^{2} / \mathrm{ml}\right)$ \\
$L_{S}$ & 0.0005 & Inertance of blood in Aorta \\
\hline Valves & & \\
$D_{M}$ & & Mitral valve \\
$D_{A}$ & & Aortic valve \\
\hline
\end{tabular}

In the above expression, $E_{n}\left(t_{n}\right)$ is the normalized elastance, $t_{n}=\frac{t}{T_{\max }}, T_{\max }=0.2+$ $0.15 T_{C}$ and $T_{C}$ is the cardiac cycle interval, i.e, $T_{C}=60 / H R$, where $H R$ is the heart-rate. Notice that $E(t)$ is a re-scaled version of $E_{n}\left(t_{n}\right)$, as shown in Figure 14. The constants $E_{\max }$ and $E_{\text {min }}$ are related to ESPVR and EDPVR, respectively.

To derive the state equation for the time-varying capacitor, one can start by writing $V(t)=C(t) \cdot P(t)^{1}$, which relates volume $V(t)$ and pressure $P(t) . C(t)$ represents the timevarying capacitance. Differentiating that relationship with respect to time, yields equation

\footnotetext{
${ }^{1}$ Hydraulic variables, volume, pressure, and flow were used instead of their electrical counterparts charge, voltage, and current respectively.
} 
(4.4) as the flow in the time-varying capacitor. In the model, left ventricular compliance is represented by a time-varying capacitance as shown in equation (4.5)

$$
\begin{gathered}
Q(t)=C(t) \frac{d P(t)}{d t}+\frac{d C(t)}{d t} P(t) \\
C(t)=\frac{1}{E(t)}
\end{gathered}
$$
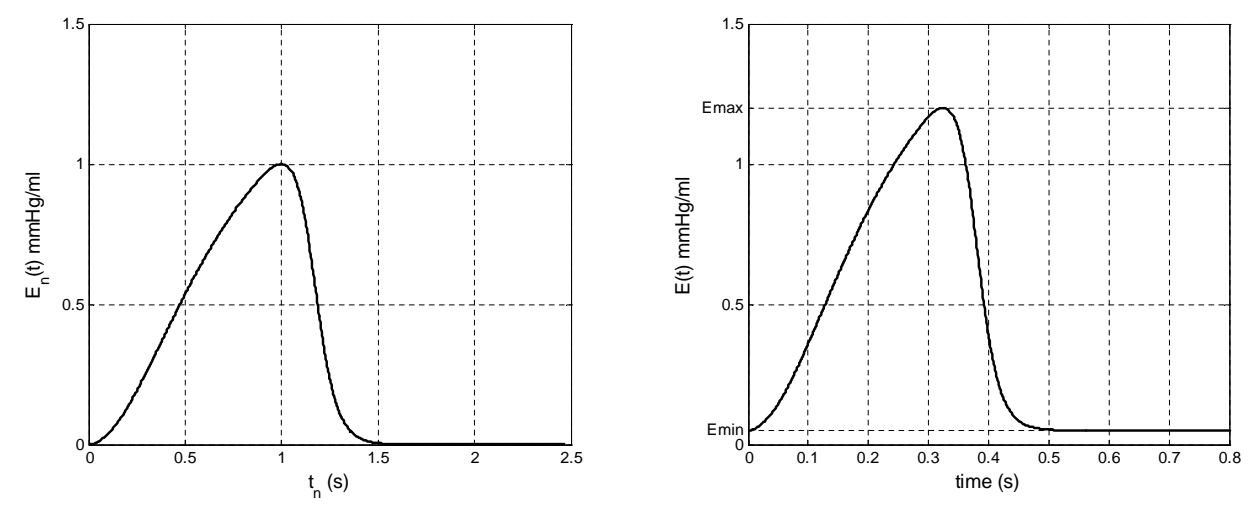

Figure 14: Normalized Elastance (left panel), Elastance $E(t)$ (right panel)

\subsubsection{Modeling the valves}

Since the circuit model of Figure 13 includes two diodes (switches representing the valves in the left side of the heart) four phases will occur, over four different time intervals, as illustrated in Table 3. Since the Isovolumic phase happens twice, this implies that we have three sets of differential equations to describe the model. However, by appropriately modeling the diodes as nonlinear elements, it is possible to write only one set of state equations that describes the behavior of the system for the four phases. Using the standard model for an ideal diode in series with a resistor, we can express the currents through the two diodes $i_{M}$ and $i_{A}$, using the expressions: 


$$
\begin{aligned}
& i_{M}=\frac{1}{R_{M}} \boldsymbol{r}\left(x_{2}-x_{1}\right)= \begin{cases}\frac{x_{2}-x_{1}}{R_{M}}, & \text { if }\left(x_{2}-x_{1}\right) \geq 0 \\
0, & \text { otherwise; }\end{cases} \\
& i_{A}=\frac{1}{R_{A}} \boldsymbol{r}\left(x_{1}-x_{4}\right)= \begin{cases}\frac{x_{1}-x_{4}}{R_{A}}, & \text { if }\left(x_{1}-x_{4}\right) \geq 0 \\
0, & \text { otherwise }\end{cases}
\end{aligned}
$$

where $\boldsymbol{r}(x)$ is the ramp function

$$
\boldsymbol{r}(x)= \begin{cases}x, & \text { if } x \geq 0 \\ 0, & \text { if } x<0\end{cases}
$$

Thus, the diodes are modeled as the cascade of two blocks: one is a ramp function, and another is a gain, representing the conductance of the diode, that modifies the slope of the ramp.

Table 3: Phases of the cardiac cycle

\begin{tabular}{c|c|c|c}
\hline \multirow{2}{*}{ Modes } & \multicolumn{2}{|c|}{ Valves } & \multirow{2}{*}{ Phases } \\
\cline { 2 - 3 } & $D_{M}$ & $D_{A}$ & \\
\hline 1 & closed & closed & Isovolumic contraction \\
\hline 2 & closed & open & Ejection \\
\hline 1 & closed & closed & Isovolumic relaxation \\
\hline 3 & open & closed & Filling \\
\hline- & open & open & Not feasible \\
\hline
\end{tabular}




\subsubsection{State Equations}

Using basic circuit theory (KVL, KCL, etc.), the state equations for the nonlinear, timevarying cardiovascular circuit model (see Figure 13) were derived as follows:

$$
\begin{aligned}
\dot{\boldsymbol{x}} & =f(t, x) \\
& =\boldsymbol{A}_{c}(t) \boldsymbol{x}+\boldsymbol{R}_{c}(t) \boldsymbol{r}(x)
\end{aligned}
$$

where $\boldsymbol{A}_{c}(t)$ and $\boldsymbol{R}_{c}(t)$ are $(5 \times 5)$ and $(5 \times 2)$ time-varying matrices respectively, and $\boldsymbol{r}(x)$ is a $(2 \times 1)$ vector that models the nonlinear behavior of the diodes. These matrices are

$$
\boldsymbol{A}_{c}(t)=\left[\begin{array}{ccccc}
\frac{-\dot{C}(t)}{C(t)} & 0 & 0 & 0 & 0 \\
0 & \frac{-1}{R_{S} C_{R}} & \frac{1}{R_{S} C_{R}} & 0 & 0 \\
0 & \frac{1}{R_{S} C_{S}} & \frac{-1}{R_{S} C_{S}} & \frac{1}{C_{S}} & 0 \\
0 & 0 & 0 & 0 & \frac{-1}{C_{A}} \\
0 & 0 & \frac{-1}{L_{S}} & \frac{1}{L_{S}} & \frac{-R_{C}}{L_{S}}
\end{array}\right], \quad \boldsymbol{R}_{c}(t)=\left[\begin{array}{cc}
\frac{1}{C(t)} & \frac{-1}{C(t)} \\
\frac{-1}{C_{R}} & 0 \\
0 & 0 \\
0 & \frac{1}{C_{A}} \\
0 & 0
\end{array}\right], \quad \boldsymbol{r}(x)=\left[\begin{array}{c}
\frac{r\left(x_{2}-x_{1}\right)}{R_{M}} \\
\frac{r\left(x_{1}-x_{4}\right)}{R_{A}}
\end{array}\right]
$$

Notice that the model described above is autonomous. It is driven mainly through the time-varying elastance function. Figure 15 shows an all-integrator block diagram of system (4.6). For comparison purposes and completeness, a set of equations for each phase was derived as well, and they are shown in Appendix A.

\subsection{MODEL VALIDATION}

In order to assess the capability of the proposed model to simulate left ventricular hemodynamics, tests were performed by implementing the model in MATLAB ${ }^{2}$. Simulations were done for both nominal steady state conditions and in response to perturbations of preload and afterload.

\footnotetext{
${ }^{2}$ The Math Works Inc., Natick, MA
} 


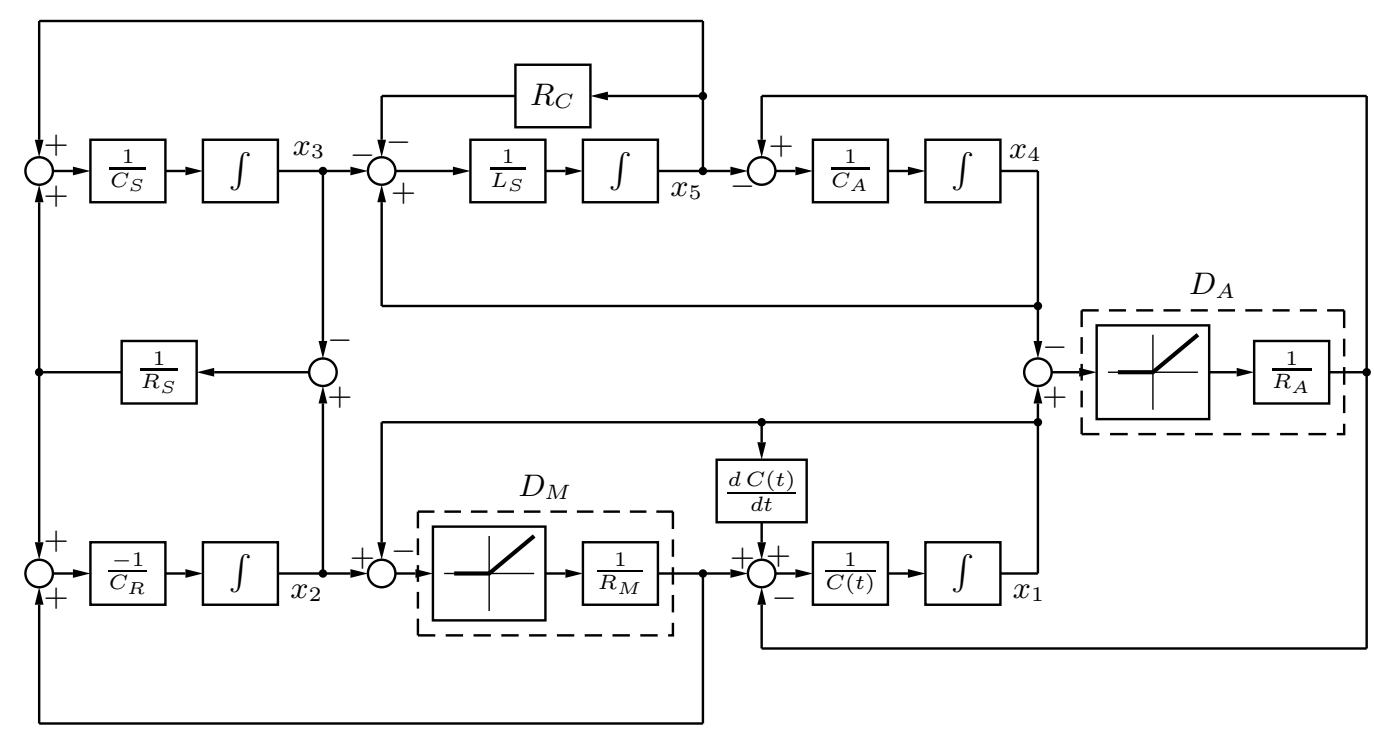

Figure 15: All integrator block diagram for the cardiovascular model

Figure 16 shows the simulation waveforms for an adult with heart rate of 75 beats per minute. The values for the elastance function, $E_{\max }=2.0, E_{\min }=0.05, H R=75 \mathrm{bpm}$ and $V_{0}=10 \mathrm{ml}$, were adapted from [41]. As a result, systolic and diastolic pressure were 117 and $77 \mathrm{mmHg}$, mean aortic pressure (MAP) was $99 \mathrm{mmHg}$, cardiac output (CO) was 5.21 l/min and stroke volume (SV) was $69.5 \mathrm{ml} /$ beat. These are consistent with hemodynamic data in normal subjects, as described in [15].

Another validation of the model is by varying preload and afterload conditions, while keeping left ventricle parameters $\left(E_{\max }, E_{\min }, V_{0}\right)$ constant. The model should produce an approximately linear relationship between end-systolic pressure and left ventricle volume, despite changes in preload and afterload. That linear relationship is known as the end systolic pressure volume relationship - ESPVR [42].

A total of four preload and four afterload conditions were simulated. In these tests, we set $E_{\max }=2.0 \mathrm{mmHg} / \mathrm{ml}, E_{\min }=0.05 \mathrm{mmHg} / \mathrm{ml}$ and $V_{0}=10 \mathrm{ml}$, as before. The resulting pressure and volume of the ventricle are depicted in the form of pv-loops. The pv-loops in Figure 17(a), represent the result of changing afterload conditions by selecting different values of systemic vascular resistance $\left(R_{S}\right)$, while keeping end diastolic volume (EDV) constant. The pv-loops in Figure 17(b) depict the result of altering preload conditions 

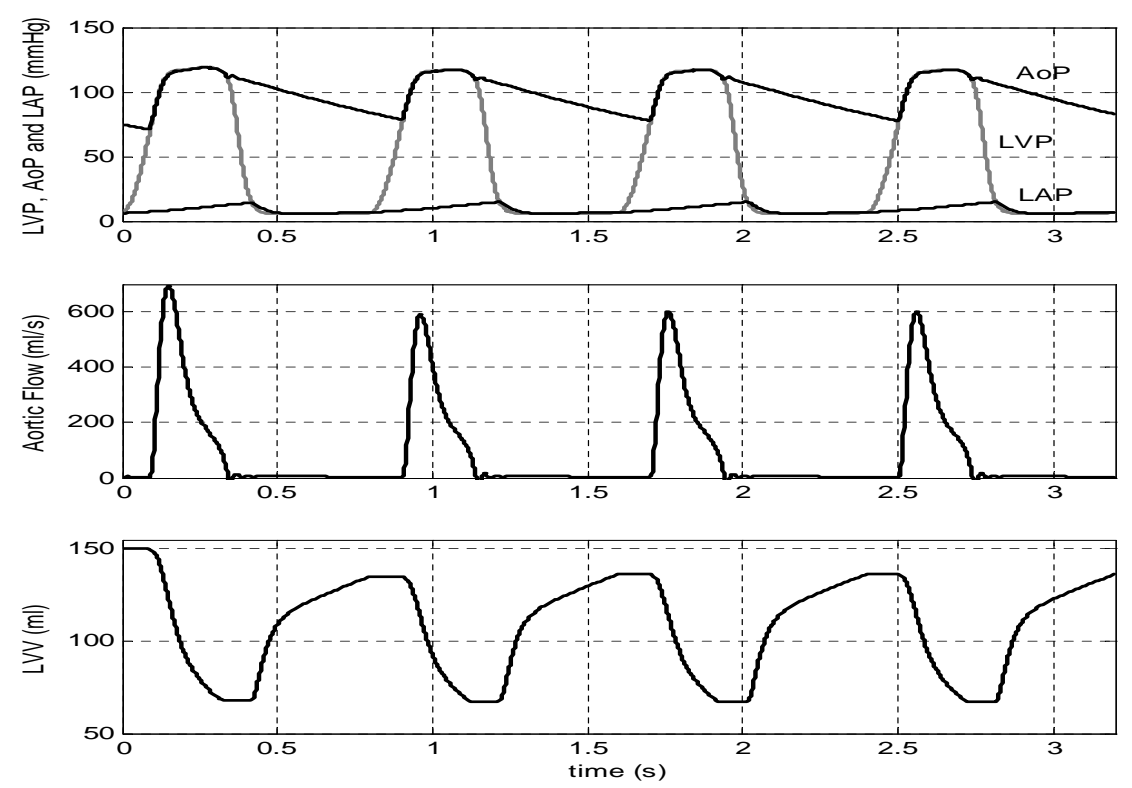

Figure 16: Simulated hemodynamic waveforms for a normal subject

by changing the mitral valve resistance $\left(R_{M}\right)$. The slope of the ESPVR $\left(E_{\max }\right)$ for the loading data in Figure $17(\mathrm{a})$ was $1.98 \mathrm{mmHg} / \mathrm{ml}$ and $V_{0}$ (volume at zero pressure) was $10.91 \mathrm{ml}$. The linear relationship between pressure and flow is evident for the ESPVR, since the correlation coefficient between those two variables was 1.0. As for preload changes (Figure 17(b)), the slope of ESPVR was $1.915 \mathrm{mmHg} / \mathrm{ml}, V_{0}$ was $8.84 \mathrm{ml}$, and the correlation coefficient was 0.99. These results show that the cardiovascular model can mimic the left ventricle behavior.

\subsubsection{Input Impedance}

The input impedance $Z_{\text {in }}$ is defined as the impedance seen by the left ventricle. It can be written as the modulus of the ratio of aortic pressure to aortic flow,

$$
\left|Z_{i n}(j \omega)\right|=\left|\frac{A o P(j \omega)}{Q_{A}(j \omega)}\right|
$$

The input impedance spectrum dictates the adequacy of the afterload model in representing the human vascular bed system. To verify this adequacy, the input impedance of the cardiovascular model determined as $Z_{i n}=Z_{1}+\left(Z_{2} \| Z_{3}\right)$, where $Z_{1}=R_{C}+j L_{S} \omega, Z_{2}=\frac{1}{j C_{S} \omega}$, 


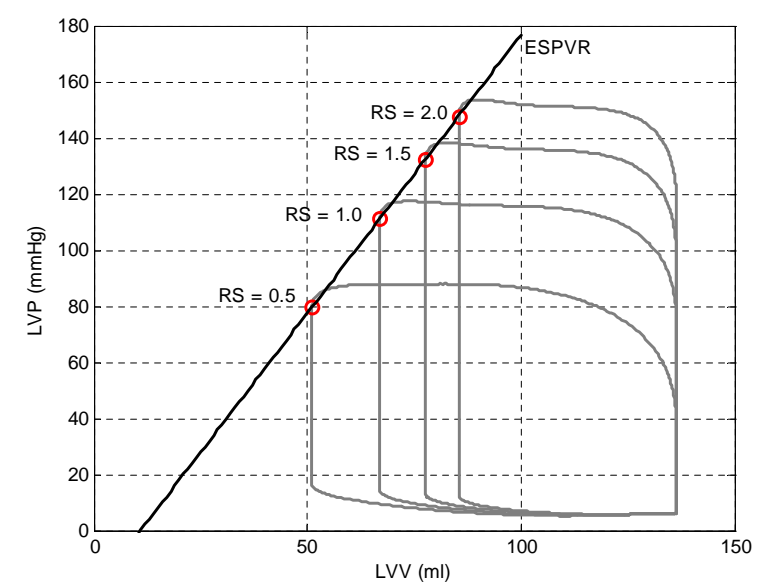

(a)

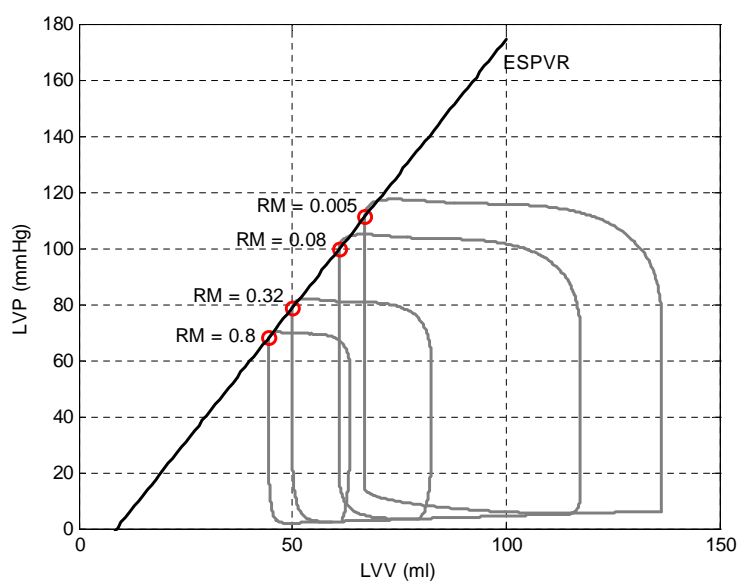

(b)

Figure 17: PV-loops used for model validation

and $Z_{3}=R_{S}+\frac{1}{j C_{R} \omega}$ (see Figure 18).

$$
\begin{aligned}
Z_{i n} & =Z_{1}+\left(Z_{2} \| Z_{3}\right) \\
& =R_{C}+j L_{S} \omega+\frac{1+j C_{R} R_{S} \omega}{j\left(C_{S}+C_{R}\right) \omega-C_{S} C_{R} R_{S} \omega^{2}} \\
& =\frac{-j L_{S} C_{R} C_{S} R_{S} \omega^{3}-\left[L_{S}\left(C_{S}+C_{R}\right)+C_{R} C_{S} R_{S} R_{C}\right] \omega^{2}+j\left[C_{R} R_{S}+R_{C}\left(C_{S}+C_{R}\right)\right] \omega+1}{j\left(C_{S}+C_{R}\right) \omega-C_{S} C_{R} R_{S} \omega^{2}}
\end{aligned}
$$

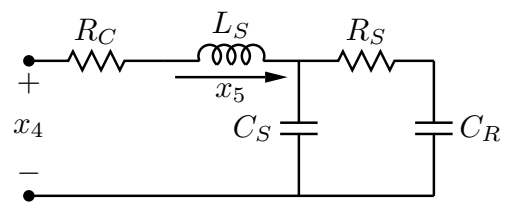

(a)

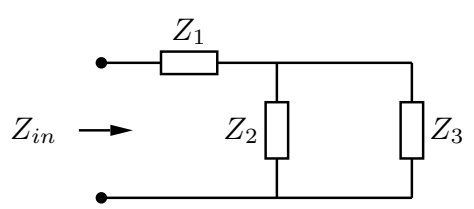

(b)

Figure 18: Input impedance of the afterload system

Figure 19 shows the input impedance $Z_{\text {in }}$ obtained from the cardiovascular model. Notice that spectrum is consistent with the spectrum obtained by Nichols et al [43] using human experimental data. 


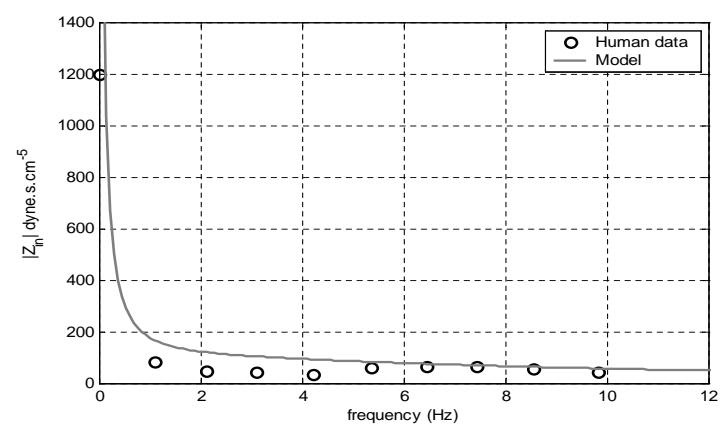

Figure 19: Input impedance spectrum

\subsubsection{Fitting Human Data}

Figure 20 shows a comparison of model simulation and human clinical data. Some model parameters were changed in this case. For instance, the value of $V_{0}$ was set at $12 \mathrm{ml}$, and $E_{\text {max }}=1.5$ was calculated from the pv-loop plot of the data. The value of $R_{S}$ was set to 0.5 in order to match the patient's hemodynamic waveform amplitudes. The other parameters are the same as in Table 2. For the LVP fitting test, a mean error of $4.4 \%$ was found. For the pv-loop test, the stroke work (area of the pv-loop, SW) of the patient, 10,700 $\mathrm{mmHg}$.ml was compared with that generated by the model, $10,500 \mathrm{mmHg} . \mathrm{ml}$, which represents a difference of $1.85 \%$.
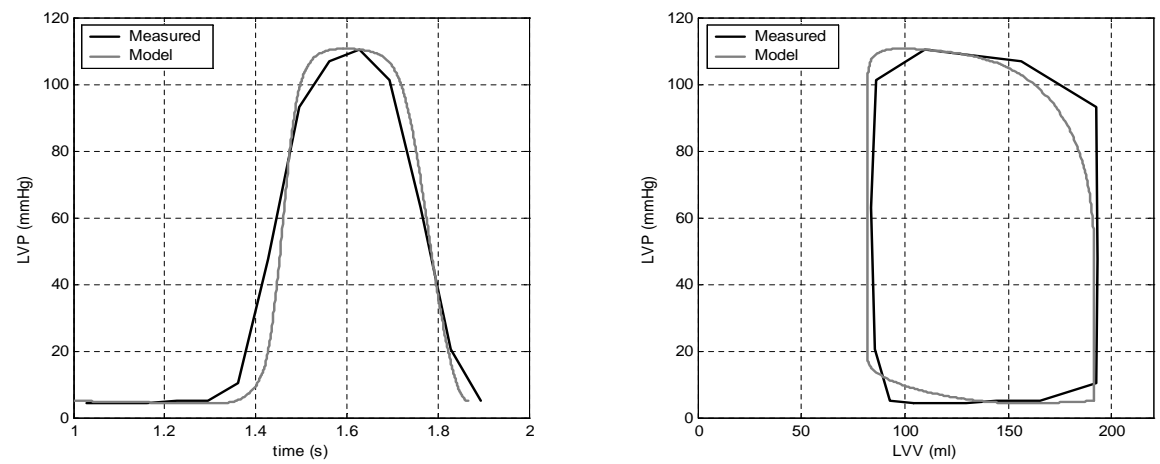

Figure 20: Curve fitting using human clinical data 


\subsection{THE COUPLED CARDIOVASCULAR-PUMP MODEL}

A model of a left ventricular assist device ${ }^{3}[45]$ was connected to the circulatory model shown in Figure 13, which assumes left ventricular cannulation. The addition of the LVAD circuit to the network adds one state variable, flow through the pump, and four passive parameters related to the cannulae. The resulting cardiovascular-pump model is shown in Figure 21.

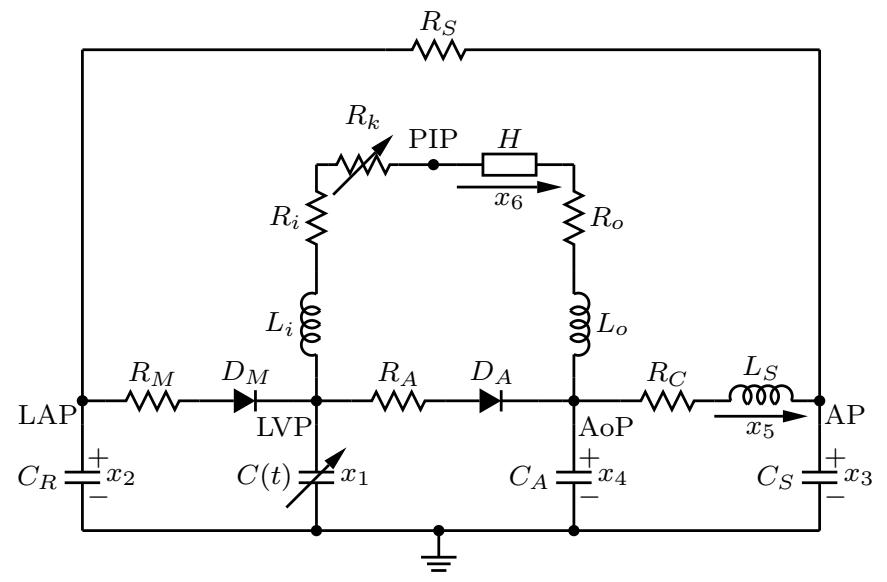

Figure 21: Cardiovascular-pump Model

Table 4 shows the state vector definition for the cardiovascular-pump model. The values of inlet and outlet resistance of the cannulae are $R_{i}=R_{o}=0.0677 \mathrm{mmHg} . \mathrm{s} / \mathrm{ml}$, respectively. The inlet and outlet inertance are $L_{i}=L_{o}=0.0127 \mathrm{mmHg} . \mathrm{s}^{2} / \mathrm{ml}$. The model equation developed for the Nimbus LVAD [39] relates the pressure difference across the pump, $H$, as a function of pump flow and pump speed

$$
H=\beta_{0} Q_{P}+\beta_{1} \frac{d Q_{P}}{d t}+\beta_{2} \omega^{2}
$$

where $\beta_{0}=-0.1707, \beta_{1}=-0.02177$ and $\beta_{2}=0.0000903$ are the pump model parameters. The resulting model is a forced system, where the primary control variable is the pump speed.

\footnotetext{
${ }^{3}$ Nimbus Inc, Rancho Cordova, CA
} 
Table 4: State Variables of the cardiovascular-pump model

\begin{tabular}{c|l|l}
\hline Variables & Name & Physiological Meaning (unit) \\
\hline$x_{1}$ & LVP & Left Ventricular Pressure $(\mathrm{mmHg})$ \\
\hline$x_{2}$ & $L A P$ & Left Atrial Pressure $(\mathrm{mmHg})$ \\
\hline$x_{3}$ & $A P$ & Arterial Pressure $(\mathrm{mmHg})$ \\
\hline$x_{4}$ & AoP & Aortic Pressure $(\mathrm{mmHg})$ \\
\hline$x_{5}$ & $Q_{T}$ & Total flow $(\mathrm{ml} / \mathrm{sec})$ \\
\hline$x_{6}$ & $Q_{P}$ & Pump flow $(\mathrm{ml} / \mathrm{sec})$ \\
\hline
\end{tabular}

\subsubsection{Suction element model}

Schima et al. [46] developed the first suction element model. It is an empirical model that can simulate suction patterns observed in pressure and flow hemodynamic waveforms of in-vivo animal studies. Originally developed for atrial cannulation, that model represents a pressure dependent-resistance, which is zero for left atrial pressures higher than a given threshold level (typically -5 to $0 \mathrm{mmHg}$ ) and increases linearly for pressures below this level at a rate of $-3.5 \mathrm{mmHg} \times \mathrm{s} / \mathrm{ml} \times 1 / \mathrm{mmHg}$.

Choi [39] used a modified version of Schima's model in his research. In this case, the resistor element varies with left ventricular pressure $\left(x_{1}\right)$, instead of left atrial pressure. The threshold, which prevents LVP from going negative, was $P_{t h}=1 \mathrm{mmHg}$. Choi's suction model was adopted in this research. In Figure 21 resistor $R_{k}$ is used to simulate suction, and its mathematical description is given as

$$
R_{k}=\left\{\begin{array}{lc}
0 & \text { if } \quad x_{1}>P_{t h} \\
-3.5 x_{1}+3.5 P_{t h} & \text { otherwise }
\end{array}\right.
$$




\subsection{STATE EQUATIONS}

The state equations for the combined cardiovascular-pump sixth order model can be written as

$$
\begin{aligned}
\dot{\boldsymbol{x}} & =f(t, x, u) \\
& =\boldsymbol{A}(t) \boldsymbol{x}+\boldsymbol{R}(t) \boldsymbol{r}(x)+b u(t)
\end{aligned}
$$

where $\boldsymbol{A}(t)$ and $\boldsymbol{R}(t)$ are $(6 \times 6)$ and $(6 \times 2)$ time-varying matrices and $b$ is a $(6 \times 1)$ constant matrix, respectively, given by the expressions:

$$
\boldsymbol{A}(t)=\left[\begin{array}{cccccc}
\frac{-\dot{C}(t)}{C(t)} & 0 & 0 & 0 & 0 & \frac{-1}{C(t)} \\
0 & \frac{-1}{R_{S} C_{R}} & \frac{1}{R_{S} C_{R}} & 0 & 0 & 0 \\
0 & \frac{1}{R_{S} C_{S}} & \frac{-1}{R_{S} C_{S}} & \frac{1}{C_{S}} & 0 & 0 \\
0 & 0 & 0 & 0 & \frac{-1}{C_{A}} & \frac{1}{C_{A}} \\
0 & 0 & \frac{-1}{L_{S}} & \frac{1}{L_{S}} & \frac{-R_{C}}{L_{S}} & 0 \\
\frac{1}{L^{*}} & 0 & 0 & \frac{-1}{L^{*}} & 0 & \frac{-R^{*}}{L^{*}}
\end{array}\right], \quad \boldsymbol{R}(t)=\left[\begin{array}{ccc}
\frac{1}{C(t)} & \frac{-1}{C(t)} \\
\frac{-1}{C_{R}} & 0 \\
0 & 0 \\
0 & \frac{1}{C_{A}} \\
0 & 0 \\
0 & 0
\end{array}\right], \quad b=\left[\begin{array}{c}
0 \\
0 \\
0 \\
0 \\
0 \\
\frac{-\beta_{2}}{L^{*}}
\end{array}\right]
$$

In the above expressions, $L^{*}$ and $R^{*}$ are defined as

$$
\begin{aligned}
& L^{*}=L_{i}+L_{o}+\beta_{1} \\
& R^{*}=R_{i}+R_{o}+R_{k}+\beta_{0}
\end{aligned}
$$

and $\boldsymbol{r}(x)$ is a $(2 \times 1)$ vector, given in 4.6, which models the nonlinear behavior of the diodes. The control variable in equation $(4.9)$ is $u(t)=\omega^{2}(t)$, where $\omega(t)$ is the rotational speed of the pump. Figure 22 shows the all integrator block diagram for the cardiovascular-pump model. 


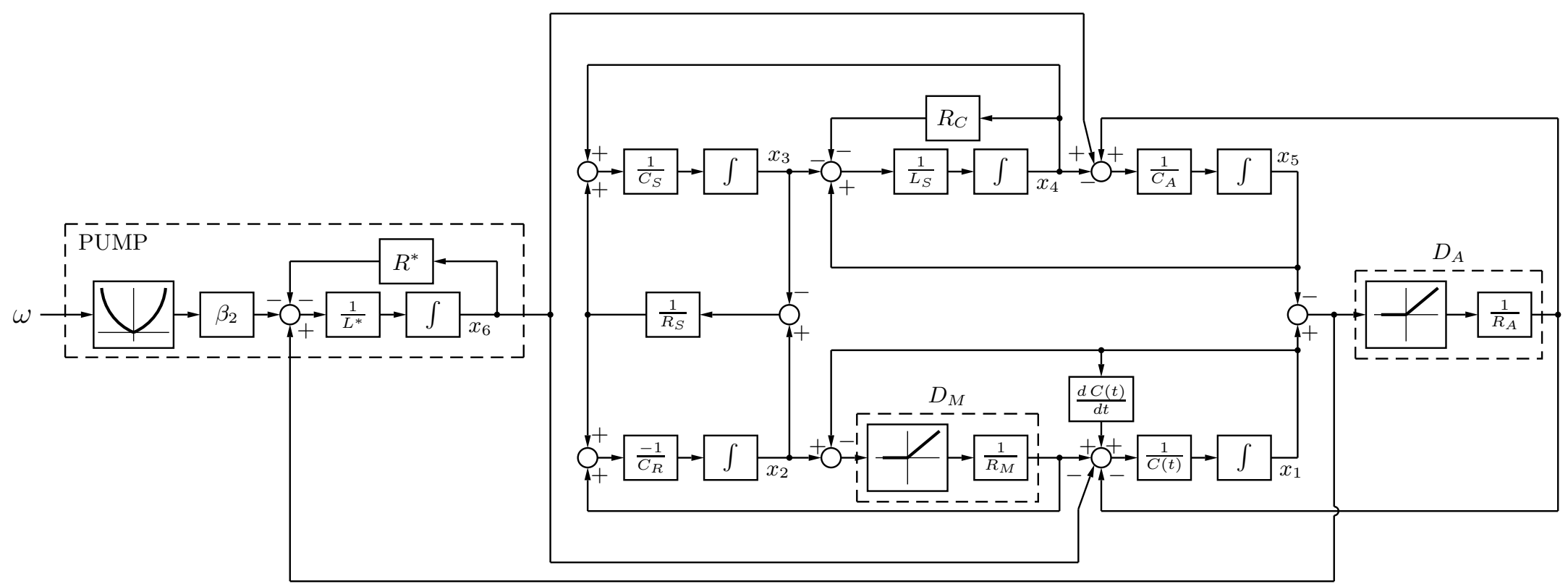

Figure 22: All integrator block diagram for the cardiovascular-pump model 


\subsection{OPEN LOOP RESPONSE}

The open loop response of the cardiovascular-pump model shown in Figure 22 was analyzed for two speed profiles: step and ramp. These profiles are commonly used during in-vivo experiments with LVADs. Since the pump is intended for sick patients, reduced $E_{\max }$ values were used in the simulation.

Because the pump provides continuous flow, cardiac output no longer can be calculated as $C O=S V * H R$. Rather, it is necessary to integrate both total flow $\left(x_{5}\right)$ and pump flow $\left(x_{6}\right)$ during one cardiac cycle $\left(t_{c}\right)$ to correctly determine the combined output of the pump and the heart. Hereafter, the term cardiac output will refer to the total flow that enters the systemic circulation, i.e.,

$$
\begin{aligned}
C O & =\text { flow through the LVAD }+ \text { flow pumped by the heart } \\
& =C O_{p}+C O_{h} \\
\int_{t}^{t+t_{c}} x_{5}(\xi) d \xi & =\int_{t}^{t+t_{c}} x_{6}(\xi) d \xi+C O_{h}
\end{aligned}
$$

To compute the cardiac contribution, one needs only to subtract $C O_{p}$ from $C O$, i.e, $C O_{h}=C O-C O_{p} . C O_{h}$ can be zero. Indeed, depending on the contractility ("strength") of the sick heart and on the pump speed, all cardiac output may be provided by the LVAD.

\subsubsection{Step response}

Two step speeds were used in this test: $9 \mathrm{krpm}$ and $12 \mathrm{krpm}$. Moreover, two different values of $E_{\max }$ were used to simulate a moderately sick heart $\left(E_{\max }=1.0\right)$ and a severely sick heart $\left(E_{\max }=0.5\right)$. Figure 23 shows the resulting cardiac output of the test. As pump speed increases, pump flow becomes less and less pulsatile, as shown in Figure 24. Since $E_{\max }$ was reduced, the LVAD provides $100 \%$ of the cardiac output, which implies that the aortic valve remains closed for all two step speeds.

The effect of pump speed on LAP, LVP, and AoP is shown in Figure 25. As pump speed increases, the ventricle is unloaded and so LVP decreases for both contractility conditions. LAP is about the same when the speeds were $9 \mathrm{krpm}$ and $12 \mathrm{krpm}$. AoP increases with pump speed, with decreasing pulsatility. 


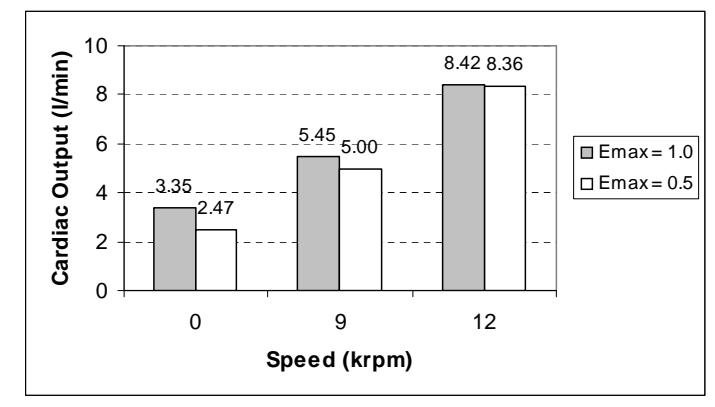

Figure 23: Cardiac Output for step speed test
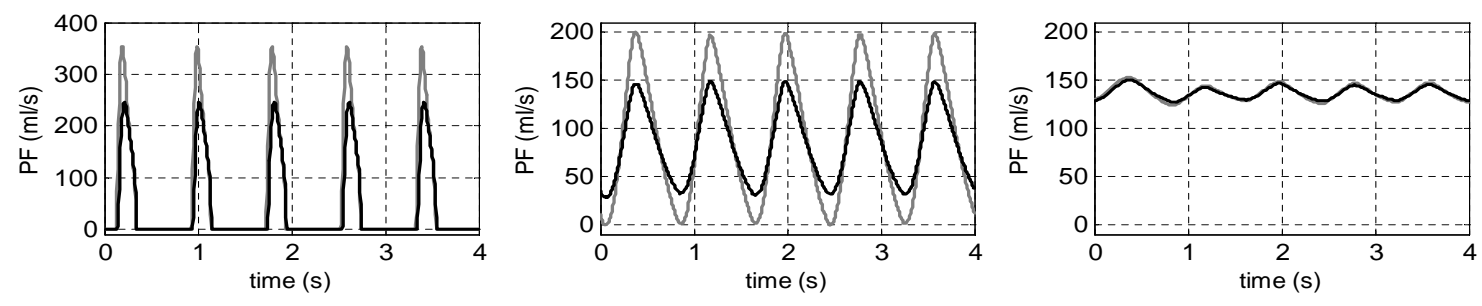

(a) $\omega=0 \mathrm{krpm}$

(b) $\omega=9 \mathrm{krpm}$

(c) $\omega=10 \mathrm{krpm}$

Figure 24: Pump flow for step speed test; dotted line: $E_{\max }=1.0$; solid line: $E_{\max }=0.5$

\subsubsection{Ramp response}

Figure 26 shows the pump speed profile used in this test. As the pump speed is increased, the amplitude of pump flow oscillation gradually decreases, while net flow increases. Beyond the point of maximum flow, the waveform exhibits sudden negative spikes, indicative of severe suction. Notice that suction occurs around $t=60 \mathrm{~s}$ for both contractility conditions. However, for the sick heart $\left(E_{\max }=1.0\right)$ there is actually aortic flow for $0 \leq t \leq 10 \mathrm{~s}$, because $Q_{T} \geq Q_{P}$ during this time interval, as shown in Figure 27(a), bottom panel.

During ejection, there are two paths for flow out of the ventricle, either through the aortic valve or through the pump. However, the aortic valve does not open if the left ventricular pressure is lower than the aortic pressure. This is usually the case, since the pump decreases the internal pressure in the ventricle. Whether the aortic valve will open or not depends on the pump speed and on how "strong" (the contractility state) the heart is [39]. In both 

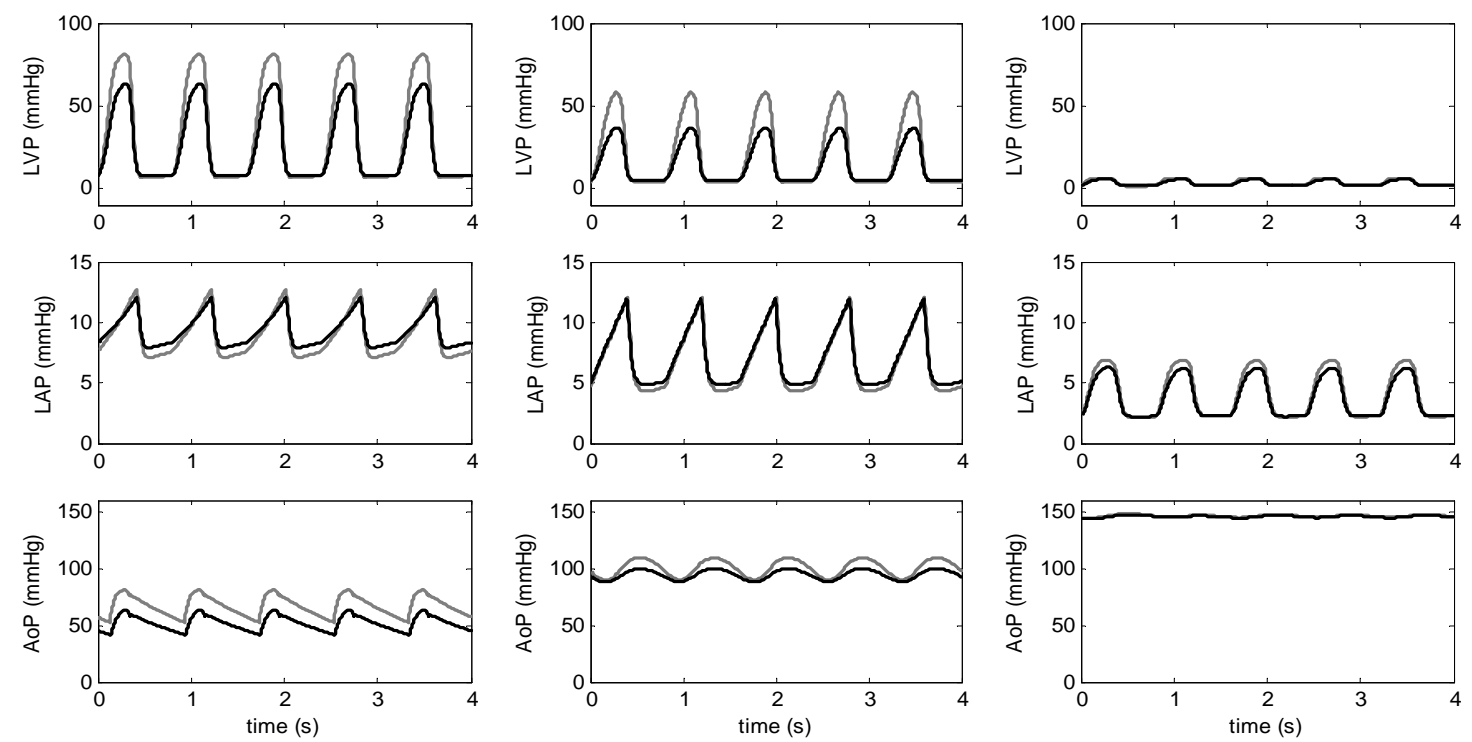

(a) $\omega=0 \mathrm{krpm}$

(b) $\omega=9 \mathrm{krpm}$

(c) $\omega=12 \mathrm{krpm}$

Figure 25: Pressures for step speed test; dotted line: $E_{\max }=1.0$; solid line: $E_{\max }=0.5$

cases, the mitral valve works as expected (opening and closing), allowing the ventricle to fill.

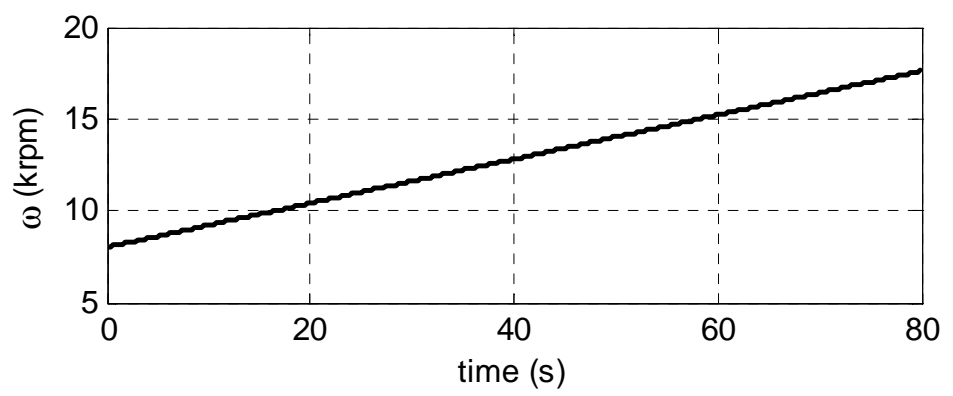

Figure 26: Ramp speed profile

\subsubsection{Comparison with experimental data}

The output of the model presented in Section 4.3 was compared with in-vivo data. These data were recorded in experiments performed on calves at the University of Pittsburgh. The simulation test was done using the same pump speed profile used in the actual in-vivo test. Model parameters were then manually adjusted to match pump flow. We set $E_{\max }=1.0$, $E_{\text {min }}=0.05, R_{S}=1.0$, and the threshold of the suction element was $P_{t h}=2.3$. Figure 28 

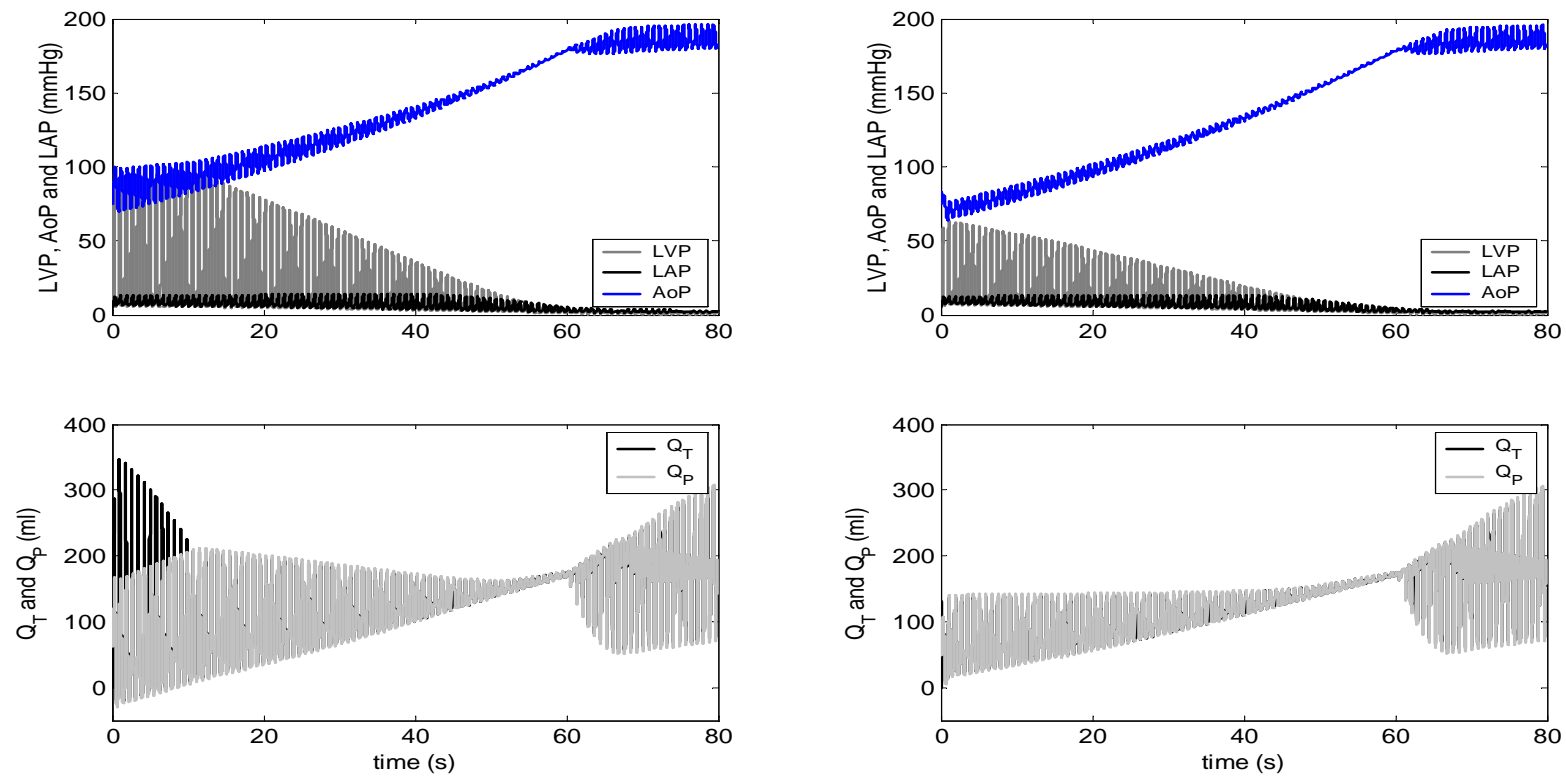

(a) $E_{\max }=1.0$

(b) $E_{\text {max }}=0.5$

Figure 27: Hemodynamic variables for ramp test

shows the simulation results. Panels (b) and (c) are pump flow (PF) and pump inlet pressure (PIP) from the in-vivo experiment, and panels (d) and (e) are the respective variables for the model.

The analysis of results for this kind of test simulation is usually done in a "qualitative" fashion [46]. We expect to see large negative spikes in pump inlet pressure when severe suction occurs, and reduced pump flow pulsatility as this condition is approached.

Pump inlet pressure presents large negative spikes when severe suction is occurring $(125<$ $t<155$ ). The model was able to reproduce these phenomena as expected (compare panels (c) and (e) in Figure 28). Pump flow model pulsatility is much less than that observed in the in-vivo data, as severe suction is approached $(90<t<125)$, possibly due to the threshold used for the suction resistor. However, from a qualitative stand point, the model can approximate suction patterns well enough to justify its use in this research study. 


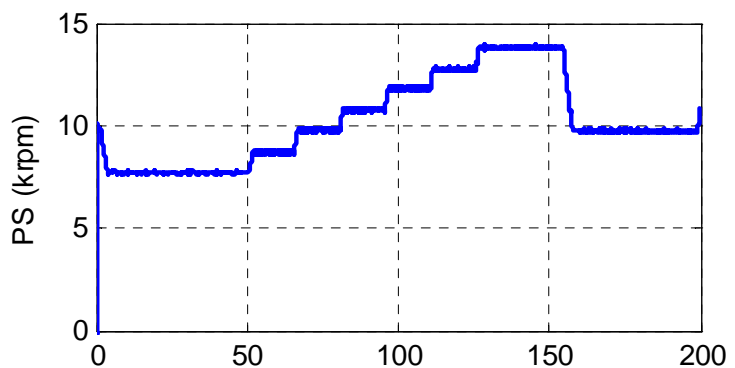

(a)
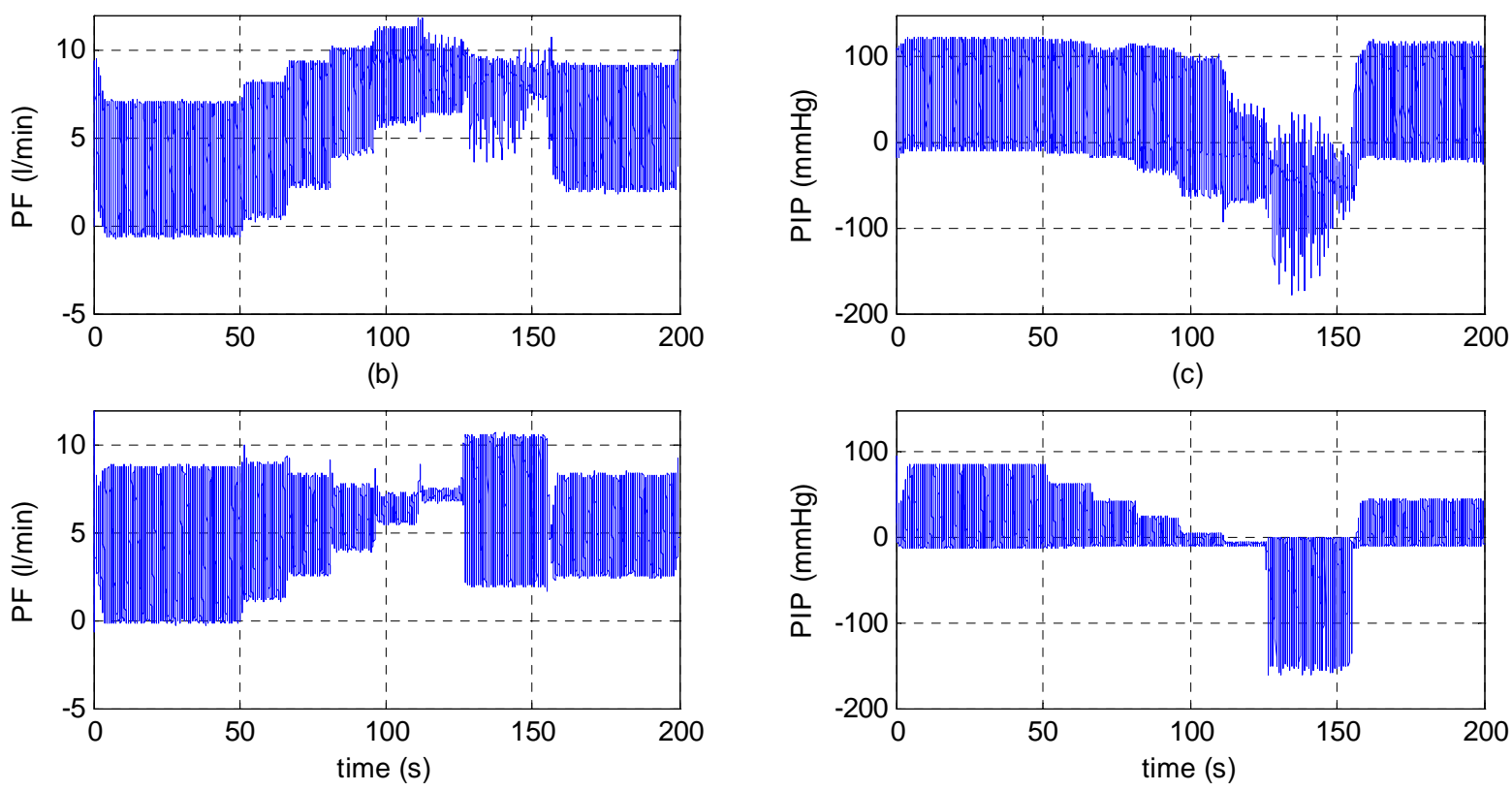

(d)

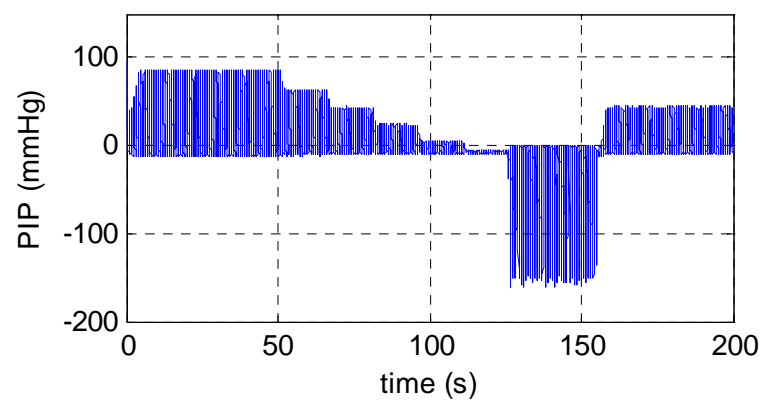

(e)

Figure 28: Simulation Results of the Cardiovascular-model. (a) Pump Speed, (b) Pump Flow from in-vivo test, (c) Pump Inlet Pressure from in-vivo test, (d) Pump Flow from model, and (e) Pump Inlet Pressure from model. 


\subsection{SUCTION DETECTION PROBLEM IN ROTARY BLOOD PUMPS}

Suction can be defined as the anatomic collapse of the ventricle. It can be due to overpumping or to contact between the cannula ${ }^{1}$ tip and the left ventricular wall (endocardium) [47]. As described earlier, suction detection is a very important problem in the control of LVADs.

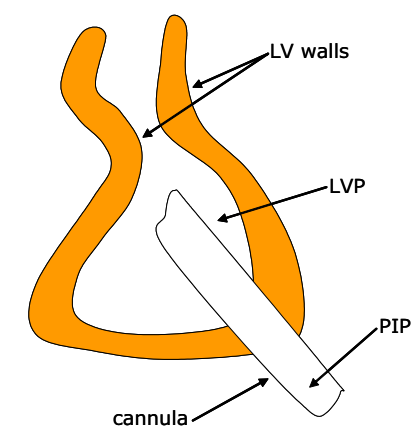

(a)

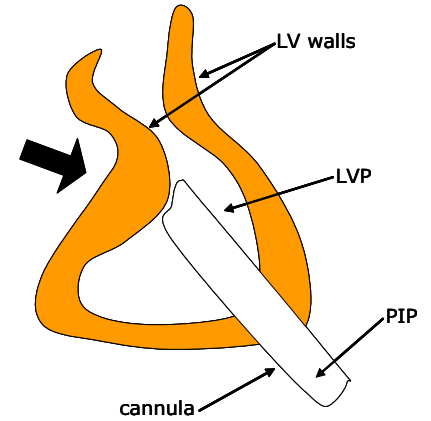

(b)

Figure 29: Pictorial representation of the suction phenomena: (a) normal, (b) suction

A new suction detection system for rotary blood pumps used in Left Ventricular Assist Devices is presented in this chapter. This system can correctly classify pump flow patterns, based on a discriminant analysis (DA) model that combines several indices derived from the pump flow signal to make a decision about the pump status. The indices considered in this approach are frequency, time, and time-frequency-domain indices [12, 13].

Figure 30 shows the proposed suction detection system. It is composed of three modules: a pre-processing module, a module that extracts features from the pump flow signal, and a decision system. The main task performed by the pre-processing module is to filter the pump flow signal, eliminating high frequency noise. This module also estimates the fundamental

\footnotetext{
${ }^{1}$ The cannula is a plastic rigid tube that connects the ventricle to the inlet of the rotary pump.
} 
frequency of pump flow. The extract-features module calculates the indices that will be used by the decision system. Finally, the decision system identifies the pump status.

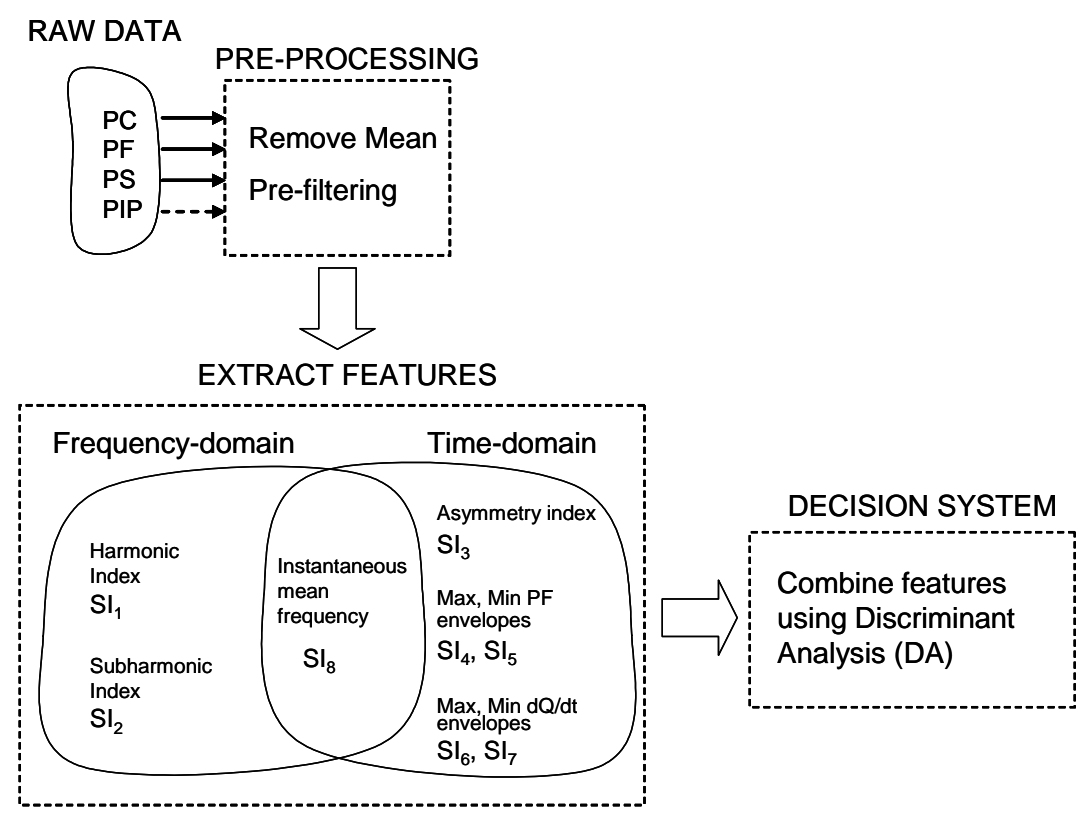

Figure 30: Schematic of the suction detection system

The chapter is organized as follows: Section 5.1 describes the indices; Section 5.2 explains the discriminant analysis method. Experimental results are presented in Section 5.3 for both two- and three-group classification problems. Section 5.4 presents simulation studies of the suction detector coupled to the cardiovascular-pump model shown in the previous chapter. Since the canonical scores may be used as control signals to set up the pump speed of the LVAD, Section 5.5 discusses how the discriminant scores behave as functions of time.

\subsection{FEATURE EXTRACTION OF PUMP FLOW}

Suction could be easily identified if reliable pressure transducers could be placed inside the left ventricle to measure left ventricular pressure (LVP) and at the head of the pump to measure pump inlet pressure (PIP). However, such an approach cannot be implemented in real time for long-term use with current technology because of problems with reliability of current pressure sensors. Due to that lack of information, most suction detection approaches 
are heuristic and depend on extraction of features from other signals that are available, such as pump flow, pump current and pump speed. Indeed, that is the main purpose of the module labeled EXTRACT FEATURES in Figure 30: to obtain features from the pump flow that can determine the pump status. To define the pump status, one can reason in terms of the resistance of the inflow track, $R_{I N}$, defined as shown in Figure 31

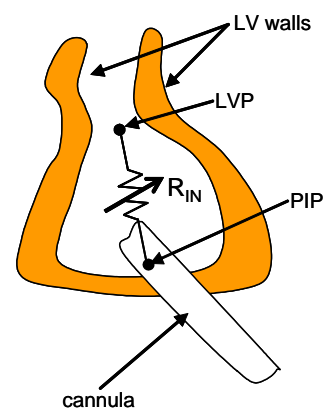

$$
R_{I N}=\frac{\Delta P}{Q_{P}}
$$

Figure 31: Illustration and definition of $R_{I N}$

where $\Delta P=L V P-P I P$ and $Q_{P}$ is the pump flow. Thus, $R_{I N}$ is a time-varying, nonlinear resistor that depends not only on the pressure difference $\Delta P$ and pump flow $Q_{P}$, but also on the position of the cannula inside the patient's heart, on pump speed, and on the contractility state of the heart. Hence, $R_{I N}$ is not constant over time, as is shown in Figure 32 , bottom.

In this research, the pump status can be one of the following: no suction (NS), moderate suction (MS), and severe suction (SS). To characterize these states, consider the experimental data shown in Figure 32. These data come from an in-vivo experiment performed on a calf, implanted with the Nimbus ${ }^{2}$ pump. Briefly, Figure 32 shows plots of four variables: pump speed (PS), left ventricular pressure and pump inlet pressure (LVP, PIP), pump flow (PF) and inflow resistance $\left(R_{I N}\right)$. Time windows $\mathrm{A}, \mathrm{B}, \mathrm{C}$ are used to illustrate how these variables change according to the three pump status. Figure 33 presents a zoomed version of time windows A, B and C, showing the ECG signal (top) and LVP and PIP (bottom). Values of diastolic $\Delta P$ were included for completeness. To define the three pump states, consider the three time windows labeled A, B, C.

\footnotetext{
${ }^{2}$ actual HeartMate II LVAD
} 
A) No Suction (NS): This state is shown in time window A. Pump Inlet Pressure (PIP) the pressure at the pump head - is positive and its difference from LVP is small (see Figure 33, A). Also, Pump Flow is relatively sinusoidal. In this particular example, $R_{I N}$ becomes negative because of the recoil of the ventricle immediately after the ejection phase. This fact may indicate an underpumping condition, in the sense that pump flow can be increased by increasing pump speed without inducing suction.

B) Moderate Suction (MS): Time window B shows this case. Some degree of suction is observed, possibly due to intermitent contact between the cannula tip and the left ventricular wall. $\Delta P$ (see Figure 33, B) and mean pump flow increase as pump speed increases. The overall net effect is a decrease in $R_{I N}$. As a consequence, the flow drawn by the pump tends to match the flow coming back to the heart (venous return);

C) Severe Suction (SS): In this case PIP presents negative spikes and PF no longer has a sinusoidal form synchronized with the patient's heart rate, as shown in time window C. The ventricle is completely unloaded and cannot support the negative pressures $(\Delta P \geq$ $15 \mathrm{mmHg}$, see Figure $33 \mathrm{C}$ ) imposed by the pump. In this case, LVP is nearly zero and $\mathrm{PIP} \leq 15$. Since cardiac tissue damage may occur, this condition should be avoided. $R_{I N}$ increases because pump flow is reduced due to the obstruction of the inflow track. Yet, pump flow does not go zero. There are two reasons to explain this phenomena. First, it is difficult to completely obstruct the cannula tip, due to its geometric construction and diameter. Second, even if this could happen, the cannula has small orifices in its side walls, which ensure that some flow is still provided by the pump.

Considering the same time windows previously defined, let us examine the behavior of $R_{I N}$ as a function of pump flow. Figure 34 shows the ECG signal (top panels) and inflow resistance (bottom) for each time window. From these plots, we notice that $R_{I N}$ defines a family of curves, due to the cardiac cycle repetition. The dark circle points are values of $R_{I N}$ during the diastolic portion of the cycle. During that phase, the heart is less active and these points are approximately clustered. Figure 35 shows the values of inflow resistance averaged over the diastolic periods for each time window. This result shows that mean inflow resistance increases as pump state changes from NS to SS. Using the inflow resistance as a parameter, the general relationship shown in equation 5.1 can be derived, which means 
that, physiologically, pump states NS and MS present some similarities, but they are quite different from pump state SS.

$$
\left.\left(R_{I N}\right)\right|_{M S} \leq\left.\left(R_{I N}\right)\right|_{N S}<<\left.\left(R_{I N}\right)\right|_{S S}
$$
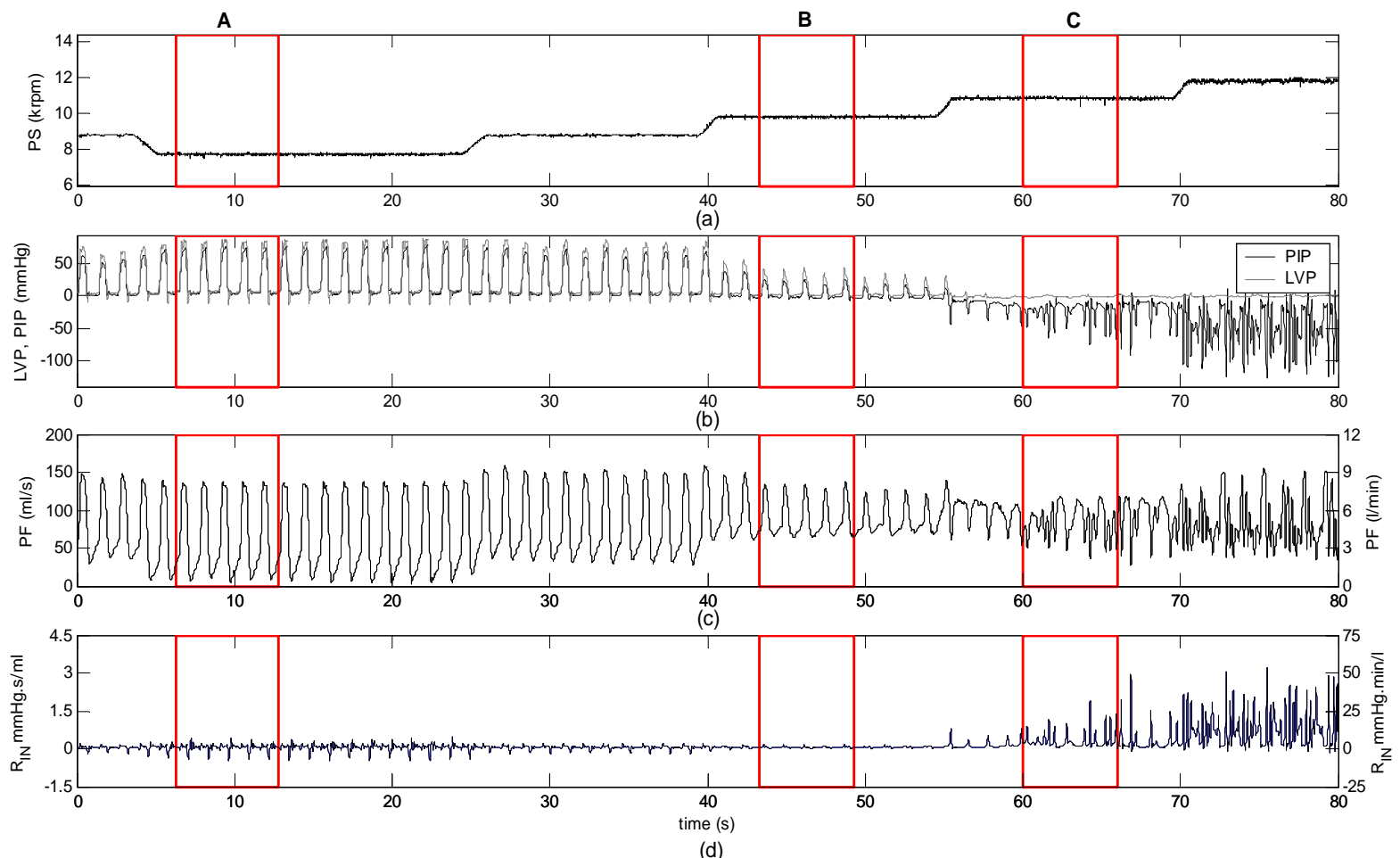

Figure 32: Experimental Nimbus data
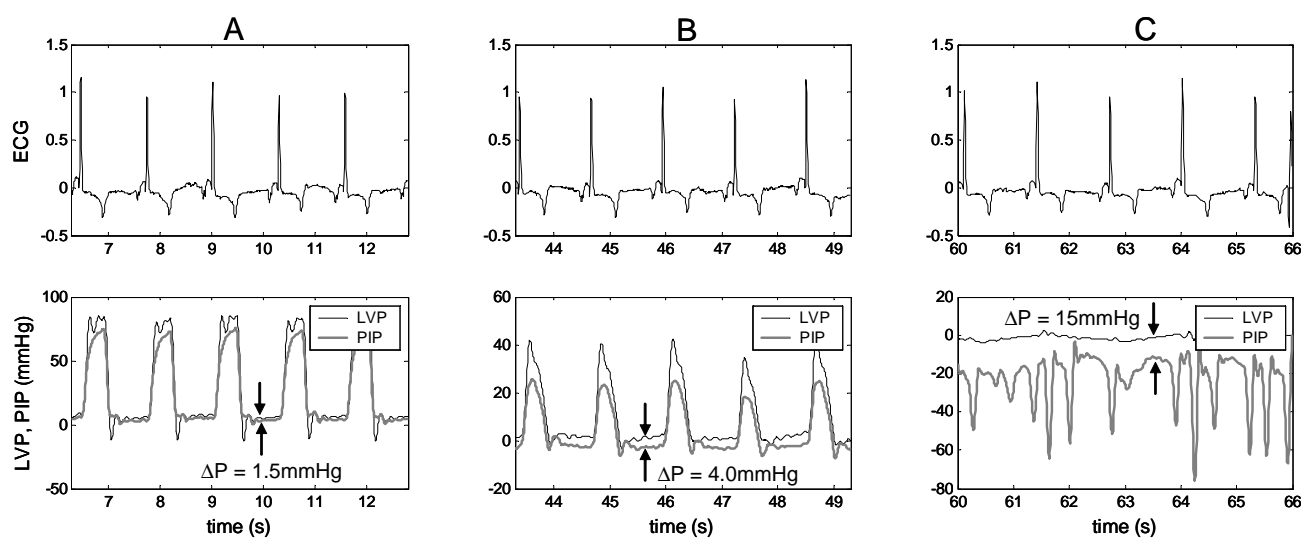

Figure 33: Hemodynamic variables for the three pumping states 

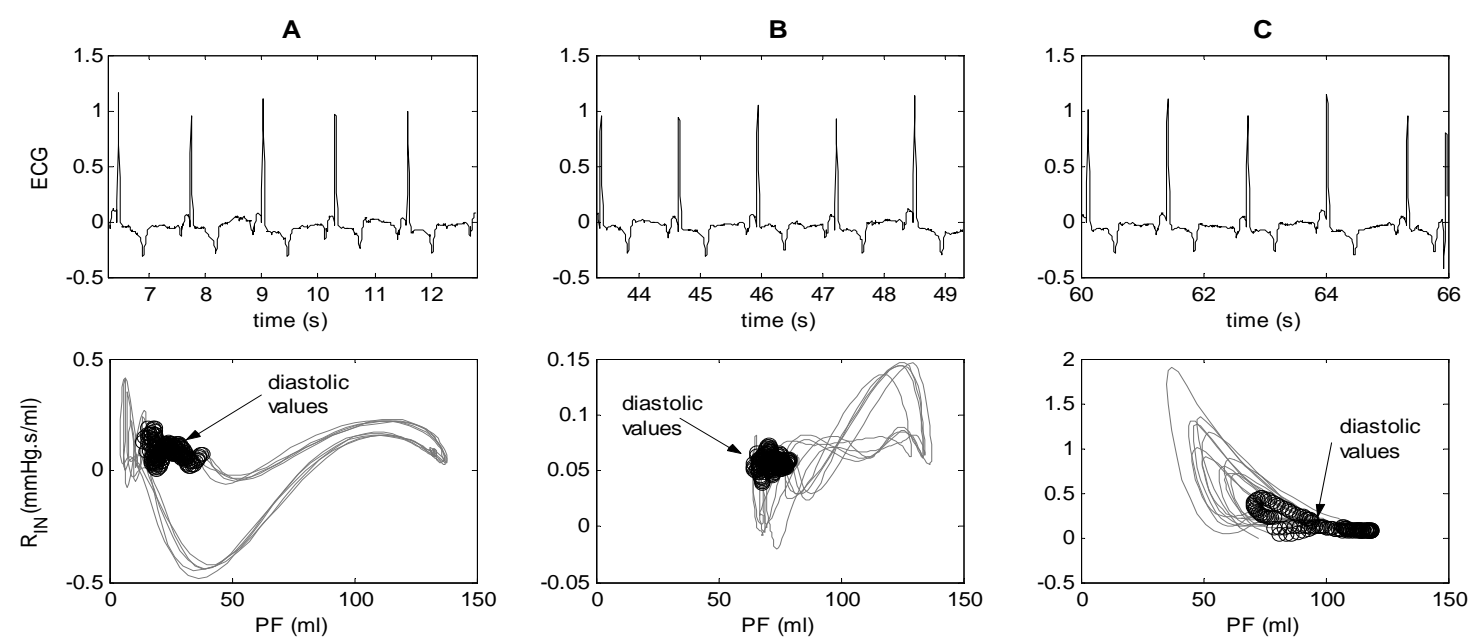

Figure 34: Inflow resistance $R_{I N}$

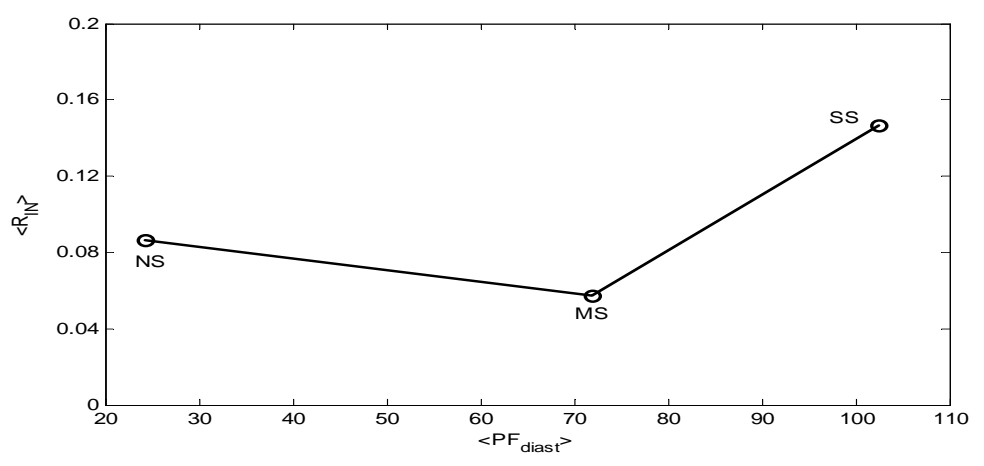

Figure 35: Mean diastolic inflow resistance

\subsubsection{The window length issue}

Most suction detection systems reported in the literature extract features from a given number of samples of pump flow or other available signal. For instance, Vollkron et al. [11] use a 5 seconds long window. Karantonis et al. [66] use a 6 seconds long window and Morello [49] uses a 2 seconds long window. Due to the necessity of buffering the signal prior to extracting the features, a certain delay will be inherent to such systems. No "ideal" length or technique to select a window length has been reported in the literature.

The shorter the window, the faster information about the pump status will be available. However, very short windows may not provide meaningful features. For instance, frequency based features usually are defined based on the signal's frequency spectrum. A short window may give poor resolution in frequency, compromising the quality of such indices. Conversely, 
a very long window would be prohibitive if real time operation of the system is intended. In this research, the window duration is based on a certain number of heart beats, rather than a fixed number of seconds.

Considering the frequency range of the human heart, a 2 seconds long window may not be suitable for a heart rate of $50 \mathrm{bpm}$, since less than 2 beats are available for feature extraction. A five heart beats long window is used in this research. This length was selected to assure that enough samples are available to calculate the indices, and also to minimize the delay present in this system. The advantage of using a window based on a certain number of beats is that the features will be calculated based on the same number of beats. Since this window duration depends on the heart rate, it is possible to have different time durations, as oppose to the case of constant time duration in seconds.

In the following sections, the frequency, time and time-frequency indices (features) derived from pump flow are described. The frequency domain indices $S I_{1}$ and $S I_{2}$ are related to the variation in energy of the harmonic and subharmonic frequency bands of the pump flow spectrum respectively. Time features encompass five indices. $S I_{3}$ is an asymmetry index; $S I_{4}$ and $S I_{5}$ measure the amplitude variation in the maximum and minimum envelopes of the pump flow signal, respectively. $S I_{6}$ and $S I_{7}$ are the maximum and minimum amplitude of the maximum and minimum envelopes of the first derivative of pump flow. Thus, indices $S I_{4}-S I_{6}$ capture pulsatility in the pump flow signal. All time indices are normalized with respect to the peak-to-peak amplitude of pump flow in order to minimize the effect of amplitude variation of the pump flow signal on the time indices. The time-frequency index, $S I_{8}$ detects variations in the standard deviation of instantaneous mean frequency of pump flow.

\subsubsection{Frequency based suction indices}

Let $Q_{P}(\omega)$ be the Fourier transform of the pump flow signal, $q_{P}(t)$, which is assumed to be approximately periodic, and $\omega_{0}$ be its fundamental frequency. Consider the frequencies $\omega_{1}=\omega_{0}-\omega_{c}$ and $\omega_{2}=\omega_{0}+\omega_{c}$, where $2 \omega_{c}$ defines an interval centered at $\omega_{0}$. Figure 36 illustrates the fundamental energy component in the magnitude spectrum of pump flow, for 
$\omega_{0}=1.0 \mathrm{~Hz}$ and $\omega_{c}=0.5 \mathrm{~Hz}$. The Harmonic index $S I_{1}$ is defined as the ratio of the total energy in the fundamental component frequency band to the total energy in the harmonic components frequency band i.e,

$$
S I_{1}=\frac{\int_{\omega_{1}}^{\omega_{2}}\left|Q_{P}(\omega)\right| d \omega}{\int_{\omega_{2}}^{\infty}\left|Q_{P}(\omega)\right| d \omega}
$$

The Subharmonic index $S I_{2}$ is defined as the ratio of the signal's subharmonic energy to the fundamental energy, i.e,

$$
S I_{2}=\frac{\int_{0}^{\omega_{1}}\left|Q_{P}(\omega)\right| d \omega}{\int_{\omega_{1}}^{\omega_{2}}\left|Q_{P}(\omega)\right| d \omega}
$$

As will be shown later, when suction occurs, energy shifts from the fundamental frequency band to the harmonic and subharmonic bands in the pump flow spectrum. Since $S I_{1}$ is directly proportional to the amount of energy in the fundamental frequency band, and $S I_{2}$ varies inversely with that quantity, we expect $S I_{1}$ to decrease and $S I_{2}$ to increase as suction occurs.

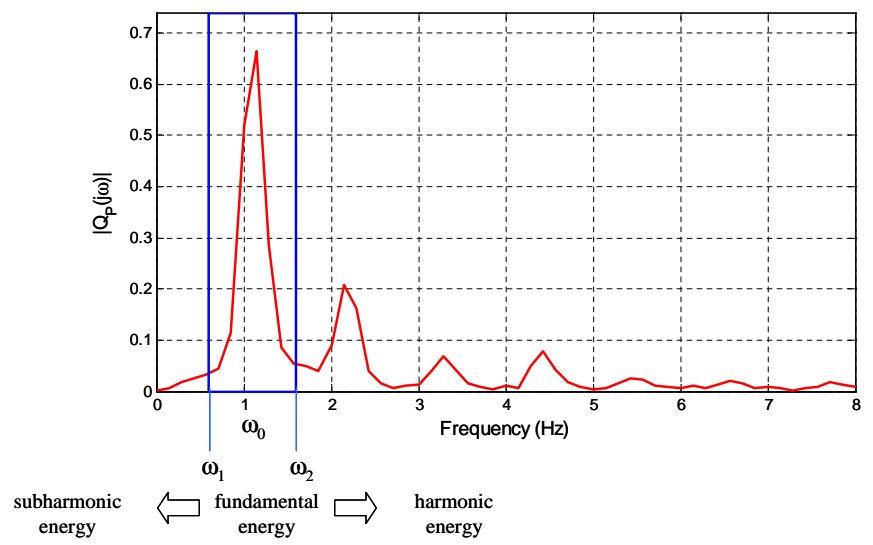

Figure 36: Spectrum of pump flow

The fundamental frequency $\omega_{0}$ is estimated from the pump flow frequency spectrum. Before the harmonic and subharmonic indices are calculated, we remove the mean and then low pass filter the pump flow signal $q_{P}(t)$ to eliminate high frequency noise. This operation makes the signal band limited to a frequency range from 0 to $10 \mathrm{~Hz}$.

Figure 37 shows an example of the pump flow wave form of an in-vivo test performed in a calf. The Levacor ${ }^{3}$ LVAD was used in this in-vivo study. (This data record will be used

\footnotetext{
${ }^{3}$ WorldHeart, Inc., formerly MedQuest, Inc., Salt Lake City, UT
} 
to illustrate the behavior of all indices.) Under desired conditions, i.e, not in a speed range that would cause suction, pump inlet pressure is positive (see Figure 37, (a)). In addition, pump flow (see Figure 37, (b)) is periodic, as depicted in the time window A. This implies that we should expect most of the energy in the PSD of the signal to be concentrated around the fundamental frequency band, $\left[\omega_{1}, \omega_{2}\right]$. Therefore, in that scenario, we have $S I_{1}>S I_{2}$.

As we approach suction (see Figure 37, time window B), the energy of the fundamental decreases and the energy of both the harmonic and subharmonic bands increase. This implies $S I_{1}$ starts to decrease and $S I_{2}$ starts to increase. When a suction event actually occurs, the energy in the fundamental component reaches its smallest value and again the energy of both the subharmonic and harmonic bands increase. The increased energy in the subharmonic band may be due to disturbances in the respiratory system caused by suction. As a consequence, $S I_{1}<S I_{2}$ (see Figure 37, time window C) when suction is occurring.

Figure 38 shows results of applying the harmonic and subharmonic indices to the in-vivo data as in Figure 37. In this case, the indices have identified correctly the occurrence of severe suction in pump flow for $127<t<152$ (see Figure 38, (a)). Using pump inlet pressure (PIP) as a reference, this time interval coincides with that for which there are negative spikes in PIP (see Figure 37, (a)). 

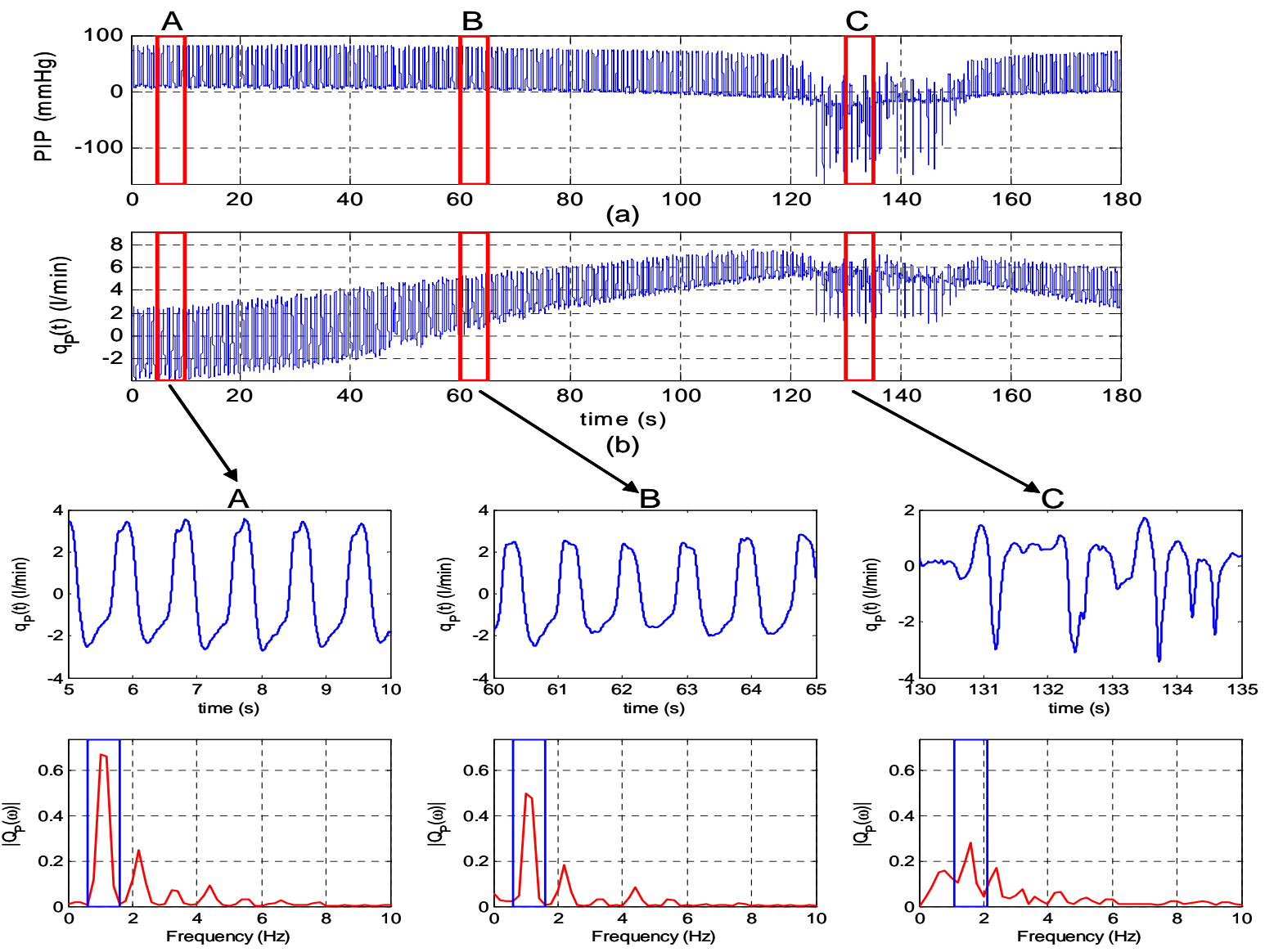

Figure 37: Example of how PSD energy changes as suction occurs; (a) Pump Inlet Pressure, (b) Pump Flow. The bottom panels are expanded segments of Pump Flow with respective spectrums. 


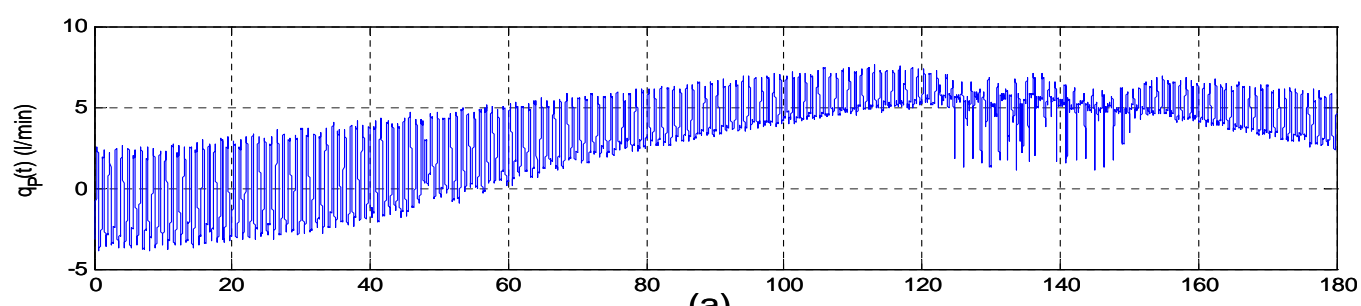

(a)

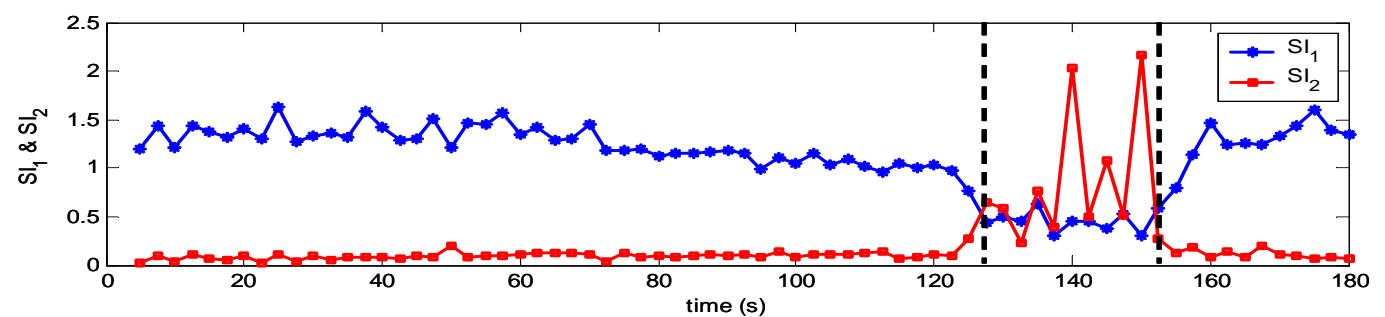

(b)

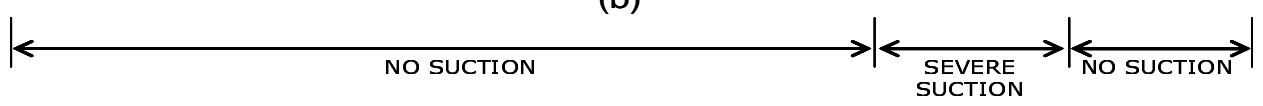

Figure 38: Simulation result of $S I_{1}$ and $S I_{2}$ to in-vivo data; (a) Pump Flow, (b) $S I_{1}$ and $S I_{2}$ as functions of time 


\subsubsection{Time based indices}

The time index $\mathrm{SI}_{3}$ measures how "symmetric" the pump flow wave form is. It is defined based on maximum and minimum envelopes of pump flow, and mean pump flow. These quantities are defined as follows. Let $M_{i}$ be the supremum of pump flow, $q_{P}(t)$, in the $\mathrm{i}^{\text {th }}$ heart beat and $m_{i}$ be the infimum. Now, consider the sequences $\left\{M_{i}\right\}_{i=1}^{N_{1}}$ and $\left\{m_{i}\right\}_{i=1}^{N_{2}}$ of all supremums and infimums of $q_{P}(t)$ in a given time window ( $\Delta t=5$ heart-beats duration), respectively. Now, assume that for $i=i^{\prime}$ sequence $\left\{M_{i}\right\}_{i=1}^{N_{1}}$ has a maximum, and that for $i=i^{\prime \prime}$ sequence $\left\{m_{i}\right\}_{i=1}^{N_{2}}$ has a minimum. We define $Q_{\max }$ and $Q_{\min }$ as:

$$
Q_{\max }=\max \left\{M_{i}\right\}_{i=1, i \neq i^{\prime}}^{N_{1}}, \quad Q_{\min }=\left\{m_{i}\right\}_{i=1, i \neq i^{\prime \prime}}^{N_{2}}
$$

The exclusion of the maximum from sequence $\left\{M_{i}\right\}$ and the minimum from $\left\{m_{i}\right\}$ is to eliminate outliers when computing the time based indices. Defining $\Delta Q_{\max }$ and $\Delta Q_{\min }$ as the amplitudes of both sequences respectively, we have:

$$
\Delta Q_{\max }=Q_{\max }-\min \left\{M_{i}\right\}, \quad \Delta Q_{\min }=\max \left\{m_{i}\right\}-Q_{\min }
$$

and $\Delta Q=Q_{\max }-Q_{\min }$ is the peak-to-peak amplitude of pump flow. Figure 39(a) shows an example of a time window with its maximum and minimum envelopes of pump flow.

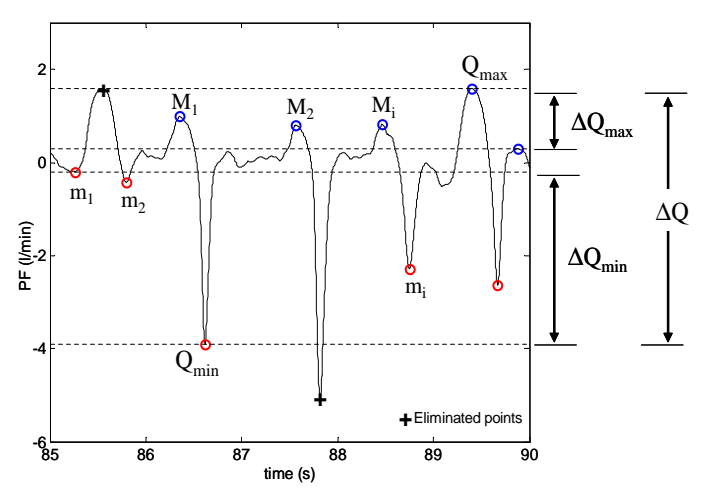

(a)

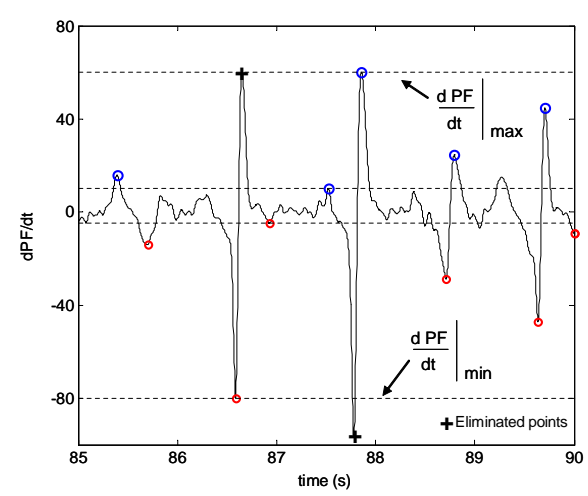

(b)

Figure 39: Example of Maximum and minimum envelopes of (a) $q_{P}(t)$, (b) $d Q_{P} / d t$

The time index $\mathrm{SI}_{3}$ is defined as

$$
S I_{3}=\frac{\bar{Q}-Q_{m i n}}{\Delta Q}
$$


where $\bar{Q}$ is the mean of pump flow. $S I_{3}$ measures how "symmetric" the time series of pump flow is. If suction is not occurring, the pump flow signal is approximately symmetric, and so $S I_{3} \approx 0.5$. When severe suction occurs, pump flow presents negative spikes, i.e. $Q_{\min }$ decreases and, as a consequence, $S I_{3}$ increases. Figure $40(\mathrm{~b})$ shows that $S I_{3} \approx 0.4$ for $t<125 \mathrm{~s}$. When severe suction occurs at $125<t<152, S I_{3}$ increases.

Time indices $S I_{4}$ and $S I_{5}$ measure the amplitude variation in the maximum and minimum envelopes of the pump flow signal. These indices are defined as

$$
S I_{4}=\frac{\Delta Q_{\max }}{\Delta Q}, \quad S I_{5}=\frac{\Delta Q_{\min }}{\Delta Q}
$$

When suction is absent, the amplitude of both indices $S I_{4}$ and $S I_{5}$ is small because pump flow is approximately periodic. This can be seen in Figure 40(c) for $t<125 \mathrm{~s}$. When severe suction occurs at $125<t<152$, both indices increase due to the spikes in the pump flow signal.
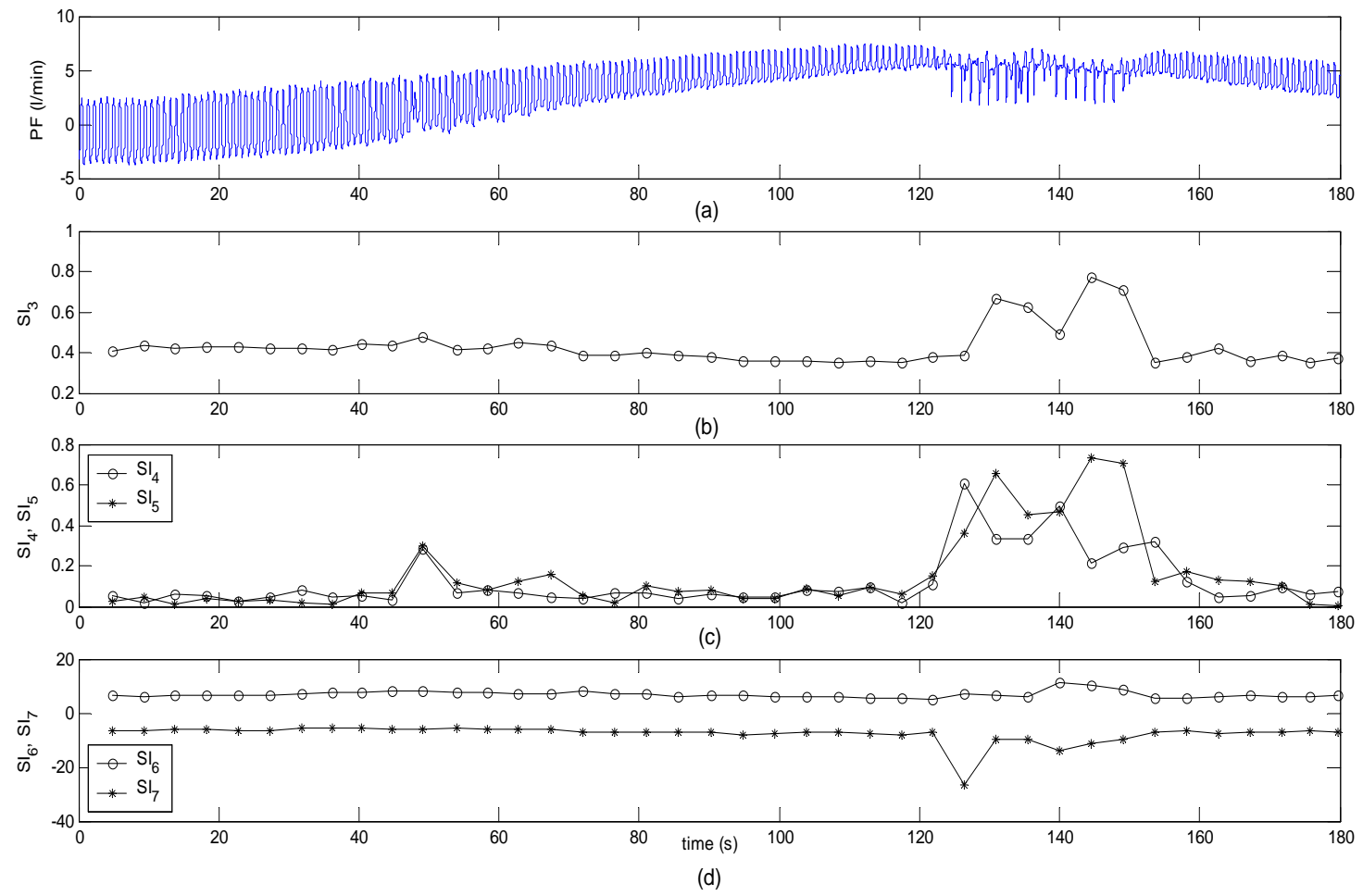

Figure 40: Simulation results of time indices to in-vivo data; (a) Pump Flow, (b) $S I_{3}$, (c) $S I_{4}$ and $S I_{5}$, (d) $S I_{6}$ and $S I_{7}$ 
To calculate $S I_{6}$ and $S I_{7}$, we first differentiate pump flow with respect to time. As was done with the pump flow signal, the maximum of $\left\{M_{i}\right\}$ and the minimum of $\left\{m_{i}\right\}$ for the derivative signal are discarded (see Figure 39(b)), to avoid outliers caused by spurious noise. Finally, $S I_{6}$ and $S I_{7}$ are calculated as

$$
S I_{6}=\frac{\left.\left(\frac{d Q}{d t}\right)\right|_{\max }}{\Delta Q}, \quad S I_{7}=\frac{\left.\left(\frac{d Q}{d t}\right)\right|_{\min }}{\Delta Q}
$$

where $\left.\left(\frac{d Q}{d t}\right)\right|_{\text {max }}=\max \left\{M_{i}\right\}$ and $\left.\left(\frac{d Q}{d t}\right)\right|_{\min }=\min \left\{m_{i}\right\}$. Figure $40(\mathrm{~d})$ shows that $S I_{6}$ increases slightly during suction, whereas $S I_{7}$ decreases at the beginning of suction. All time indices were normalized with respect to $\Delta Q$ to minimize their dependence on pump flow amplitude. Mathematically this means that these indices are not affected by scale factors used when acquiring the pump flow signal. Physiologically, normalization attempts to minimize effects due to changes in overall flow values.

\subsubsection{Time-frequency based index}

The time-frequency algorithm to detect suction events is based on the standard deviation of instantaneous mean frequency of pump flow, defined as

$$
S I_{8}=\sqrt{\operatorname{var}\left(\langle\omega\rangle_{t}^{s p}\right)}
$$

In this formulation, the instantaneous mean frequency is defined as the average frequency at a given time [50], i.e,

$$
\langle\omega\rangle_{t}^{s p}=\frac{\int \omega P_{s p}(\omega, t) d \omega}{\int P_{s p}(\omega, t) d \omega}
$$

where $P_{s p}(\omega, t)$ is the spectrogram, the squared magnitude of the Short-time Fourier Transform (STFT) defined as

$$
P_{s p}(\omega, t)=\left|\int q_{P}(\tau) h^{*}(\tau-t) e^{-j \omega \tau} d \tau\right|^{2}
$$

In equation (5.11), $h(t)$ can be interpreted as a window that selects a local section of the signal $q_{P}(t)$ for Fourier analysis. Two extreme cases can occur, depending on the choice of the window. If $h(t)$ is a very long window, a high resolution spectrogram is obtained, 
but time resolution is reduced; conversely, if $h(t)$ is a very short window, $P_{s p}$ gives a low resolution spectrogram.

As for the previous frequency indices, we assume that under desired circumstances pump flow is approximately periodic, and its fundamental frequency $\omega_{0}$ is the patient's cardiac frequency. If the window $h(t)$ is selected such that the spectrogram of pump flow presents "good" resolution in frequency, we expect to see a line parallel to the time axis in the time $\times$ frequency plane. In other words, the spectrogram of pump flow is similar to that of a pure tone signal. This implies that the average frequency at a given time $\langle\omega\rangle_{t}^{s p} \approx \omega_{0}$.

Figure 41 shows the pump flow signal (PF) (top) and three time windows, A, B and C, representing the pump status as defined in Section 5.1. For each of these windows, PF, the spectrogram of PF and the instantaneous mean frequency of PF are shown. The standard deviation of instantaneous mean frequency of pump flow is "small" when the patient is not experiencing suction $\left(S I_{8}=0.0170\right.$ in time window A) and increases as suction occurs, e.g. $S I_{8}=0.0245$ for time window B and $S I_{8}=0.3298$ in time window C. 


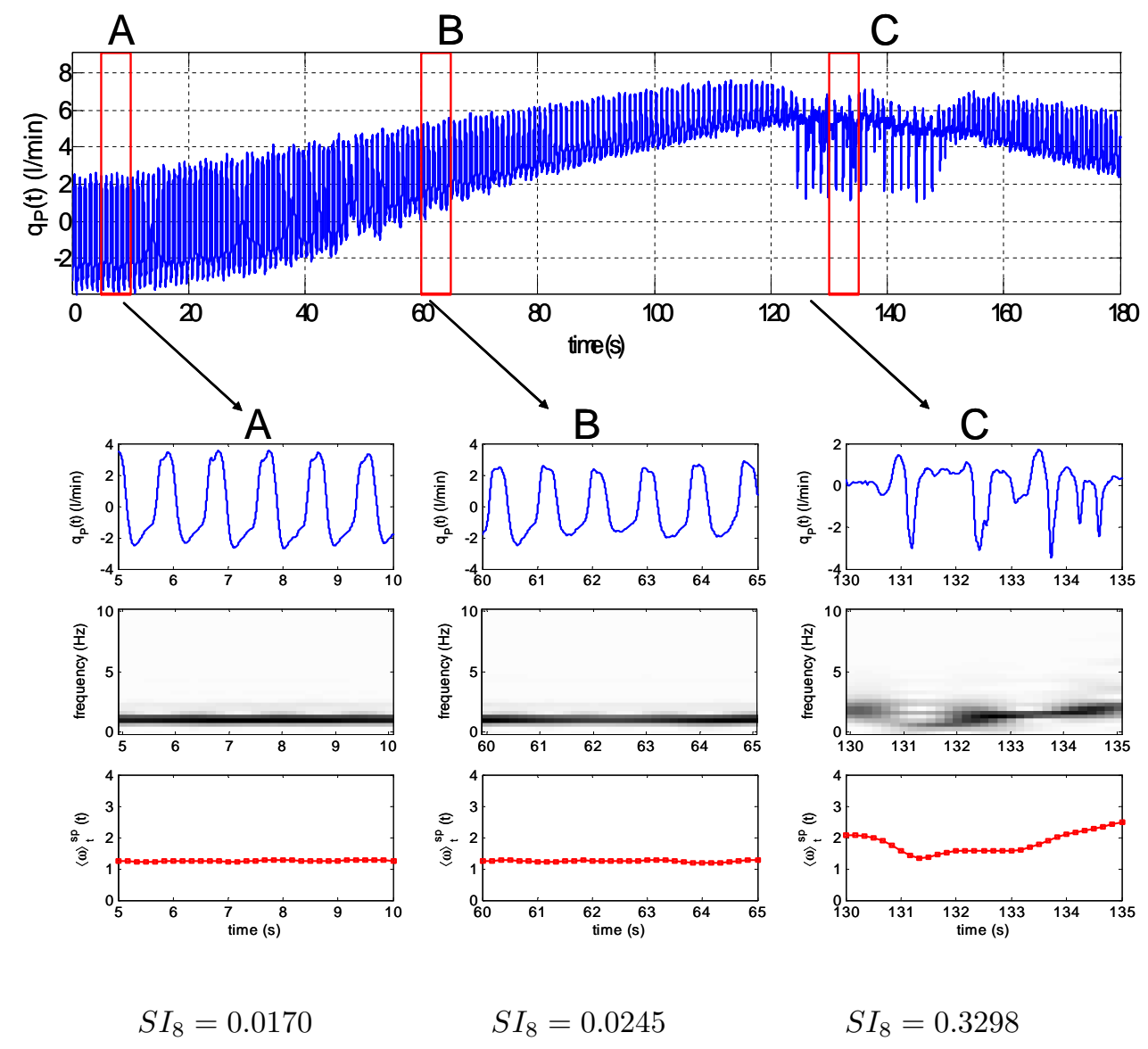

Figure 41: Spectogram results and Instantaneous frequency of Pump Flow for 3 time windows. A) No Suction, B) Moderate Suction, and C) Severe Suction. In each case, panels from the top are PF, Spectrogram of PF, and the Instantaneous mean frequency of PF, $\langle\omega\rangle_{t}^{s p}$ respectively. 


\subsection{THE DECISION SYSTEM}

The purpose of the DECISION SYSTEM module in Figure 30 is to combine the several features described in the previous section $\left(S I_{1}-S I_{8}\right)$, in order to classify a new sample ${ }^{4}$. To do so, a learning system needs to be derived. Several methods in statistical pattern recognition have been proposed to design learning systems, such as discriminant analysis, neural networks and, more recently, support vector machines. Among these, discriminant analysis is the most widely used method, for its well known mathematical foundation, robustness and relatively simple implementation. These reasons motivated us to use Discriminant Analysis (DA) to design a linear classifier.

Discriminant analysis is a dependent method like analysis of variance (ANOVA). However, in this case, the dependent variables are categorical in nature. The major objective of discriminant analysis is to achieve the clearest possible separation or discrimination between or among groups $[51,52]$. In this respect, the two-group discriminant problem is a special case of multiple regression: the independent variables are used to account for as much of the variation as possible in the dependent variable. When there are more than two groups, multiple discriminant analysis (MDA), a special case of canonical correlation, is used to explain the differences between groups.

\subsubsection{The Discriminant Analysis Method}

Consider a given matrix of $n$ observations of a $p$-variate pattern, $X_{n \times p}$. Also, consider that these observations are classified in $g$ groups. Each row vector $\boldsymbol{x}$ of the matrix $X$ denotes an observation.

The discriminant analysis method based on Fisher's approach consists of finding a linear combination of the independent variables (predictors) $\boldsymbol{x}^{T} \boldsymbol{a}$ that would produce "maximally different" discriminant scores ${ }^{5}$ across groups. Figure 42 illustrates this idea for the two-group classification problem.

\footnotetext{
${ }^{4} \mathrm{~A}$ sample is a vector whose components are the indices, i.e., $x=\left[S I_{1}, S I_{2}, \cdots, S I_{8}\right]$, calculated for each time window.

${ }^{5}$ These are the projections of the predictors in the space spanned by the discriminant functions.
} 


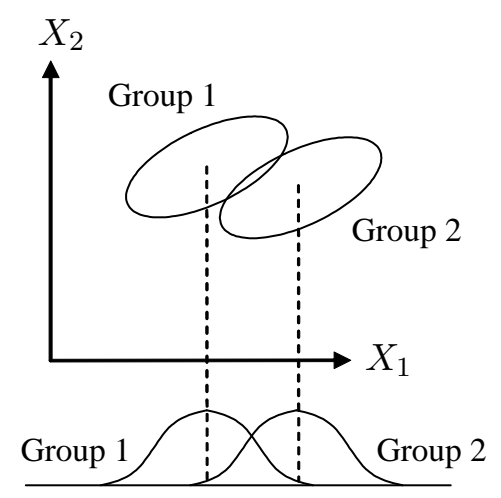

(a)

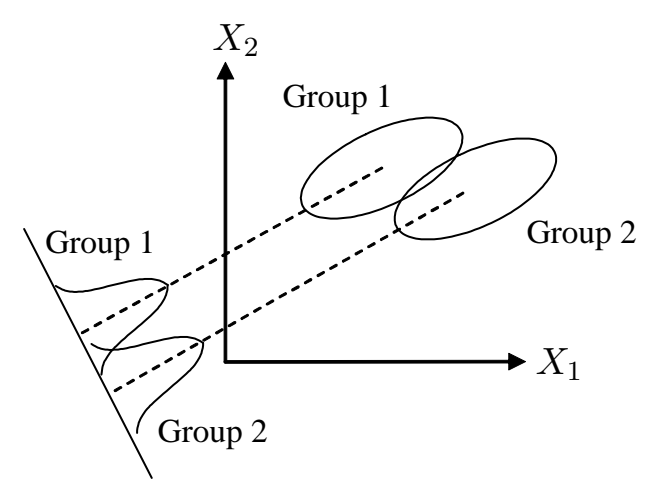

(b)

Figure 42: Fisher's idea for discriminant analysis. In (a), $X_{1}$ is used to discriminate between groups 1 and 2; in (b), a linear combination of $X_{1}$ and $X_{2}$ does a better result.

Formally, the general classification problem in DA can be stated as an optimization problem as

$$
\begin{array}{ll}
\max J(\boldsymbol{\alpha})= & \frac{\boldsymbol{\alpha}^{T} \boldsymbol{B} \boldsymbol{\alpha}}{\boldsymbol{\alpha}^{T} \boldsymbol{W} \boldsymbol{\alpha}} \\
\text { subject to } & \boldsymbol{\alpha}^{T} \boldsymbol{W} \boldsymbol{\alpha}=I
\end{array}
$$

where $\boldsymbol{B}$ and $\boldsymbol{W}$ are the between-group and within-group scatter matrices respectively.

Indeed, the problem defined in (5.12) is an eigenvalue problem, and the optimal $\alpha$ consists of the generalized eigenvectors that correspond to the largest eigenvalues in

$$
\left(\boldsymbol{W}^{-1} \boldsymbol{B}-\lambda \boldsymbol{I}\right) \alpha=0
$$

Note that the columns of the rectangular matrix $\boldsymbol{\alpha}$ define the coefficients of the linear combination of the predictors (features), for which the criteria $J(\alpha)$ has a maximum.

Fisher's objective was to choose $\boldsymbol{a}$ to maximize the ratio of the between-group variance to the within-group variance. These terms come from ANOVA and are defined as follows. Let $\boldsymbol{y}=\left(y_{i j}\right)$ be a variable whose values are observations of a certain experiment from different treatment groups, i.e., $y_{i j}$ represents the $i^{\text {th }}$ observation in treatment group $j$. Let $m_{j}$ denote the mean of $\boldsymbol{y}$ in group $j, \boldsymbol{m}=\left(m_{j}\right)$. Now, let $G_{n \times g}$ be the incidence matrix (i.e, $g_{i j}=1$ if 
and only if observation $i$ belongs to group $j)$. Note that $G^{T} G=\operatorname{diag}\left(n_{j}\right)$, where $n_{j}$ is the number of observations for group $j$. Then the within-group variance is defined as

$$
\begin{aligned}
W_{y} & =\frac{1}{g} \sum_{j} \frac{\sum_{i}\left(y_{i j}-m_{j}\right)^{2}}{n_{j}-1} \\
& =\frac{\|\boldsymbol{y}-G \boldsymbol{m}\|}{n-g}
\end{aligned}
$$

and the between-group variance is

$$
\begin{aligned}
B_{y} & =\frac{\sum_{j}\left(m_{j}-\bar{y}\right)^{2}}{g-1} \\
& =\frac{\|G \boldsymbol{m}-\bar{y} \mathbf{1}\|}{g-1}
\end{aligned}
$$

Equations 5.13 and 5.14 represent two different ways to estimate the variance in the observations. The basic idea in ANOVA is to make an inference about differences in group means by comparing these two different estimates of the variance. When there are no differences among group means, the two equations produce essentially the same result. The within-group variance is the average of the within-group sample variances. It captures the variability of the observation $y_{i j}$ around the sample mean $\left(m_{j}\right)$ for each group $j$. It is also possible to estimate the variance by looking across groups, i.e., by looking at the variance of the group means $\left(m_{j}\right)$ around the overall mean $\bar{y}$. Figure 43 illustrates these definitions for the variance estimates.

The "total sum of squares", $S_{T}$, is the sum squared deviations of the observations around the overall mean. $S_{T}$ can be defined as a "partitioning" of variance: the between-group sum of squares plus the within-group sum of squares, i.e.,

$$
S_{T}=\frac{(g-1) B_{y}+(n-g) W_{y}}{n-1}
$$

These definitions can be extended for the multivariate case by defining $M_{g \times p}$ as the matrix of group means and

$$
\begin{aligned}
W & =\frac{(X-G M)^{T}(X-G M)}{n-g} \\
B & =\frac{(G M-\mathbf{1} \overline{\boldsymbol{x}})^{T}(G M-\mathbf{1} \overline{\boldsymbol{x}})}{g-1}
\end{aligned}
$$




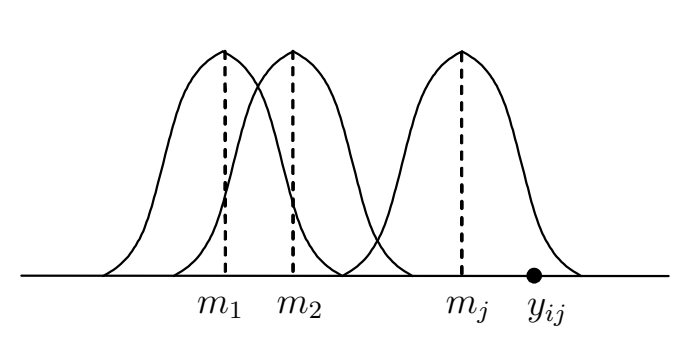

The within-group variance is an average of all group variances

$$
W_{y}=\frac{1}{g} \sum_{j} \frac{\sum_{i}\left(y_{i j}-m_{j}\right)^{2}}{n_{j}-1}
$$

(a)

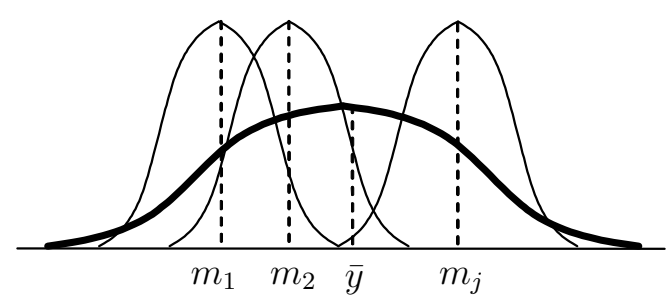

The between-group variance measures the variability of the group means around the overall mean

$$
B_{y}=\frac{\sum_{j}\left(m_{j}-\bar{y}\right)^{2}}{g-1}
$$

(b)

Figure 43: Within- and between-group variance estimates

Therefore, the linear combination $\boldsymbol{x}^{\boldsymbol{T}} \boldsymbol{a}$ has variances $a^{T} W a$ and $a^{T} B a$, and total variance

$$
a^{T} S_{T} a=\boldsymbol{a}^{T} \frac{(g-1) B+(n-g) W}{n-1} \boldsymbol{a}
$$

The classical approach to find $\boldsymbol{a}$ is to seek a rescaling of the variables $\boldsymbol{x}^{T} S$ such that their within-group covariance matrix is the identity matrix $I$ and then perform an eigendecomposition of $B$ expressed on these variables. To accomplish this, one can first rescale all variables to unit variance and then use singular value decomposition (SVD) $X=U \Lambda V^{T}$. Consider now that we are working with the rescaled variables. As stated before, the matrix $G^{T} G$ is diagonal, containing the numbers $n_{j}$ of observations on each group. Let $T=\operatorname{diag}\left(\sqrt{n / n_{i}}\right)$ so that

$$
T G^{T} G T=n I
$$

The group means are given by the $g \times p$ matrix $M=\left(G^{T} G\right)^{-1} G^{T} X$. Since $X$ has been centered, the column sums of $M$ (weighted by group size) are zero, hence $M$ has rank 
$r \leq \min (p, g-1)$.

Consider the SVD of $T^{-1} M=U \Lambda V^{T}$. Assume that neither $p$ nor $g$ is large. We will assume $U$ is $g \times r, \Lambda$ is $r \times r$ and $V$ is $p \times r$. From the SVD, we have

$$
\begin{aligned}
(g-1) B & =(G M)^{T}(G M) \\
& =\left(G T U \Lambda V^{T}\right)^{T} \cdot\left(G T U \Lambda V^{T}\right) \\
& =V \Lambda^{T} U^{T} \cdot\left(T G^{T} G T\right) U \Lambda V^{T} \\
& =V \Lambda^{T} U^{T} \cdot(n I) U \Lambda V^{T} \\
& =n V \Lambda^{2} V^{T} \\
& \\
(n-g) W & =(X-G M)^{T}(X-G M) \\
& \left.=X^{T}-M^{T} G^{T}\right)\left(X-G M^{T} X-X^{T} G M-M^{T} G^{T} X+M^{T} G^{T} G M\right. \\
& =X^{T} X-M^{T} G^{T} G M-M^{T} G^{T} G M+M^{T} G^{T} G M \\
& =X^{T} X-(G M)^{T} G M \\
& =X^{T} X-(g-1) B \\
& =n I-n V \Lambda^{2} V^{T} \\
& =n V\left[I-\Lambda^{2}\right] V^{T}
\end{aligned}
$$

where we have used the identity $M=\left(G^{T} G\right)^{-1} G^{T} X \Rightarrow G^{T} G M=G^{T} X \Rightarrow X^{T} G=$ $M^{T} G^{T} G$ in the fourth line in the derivation of $W$.

The original problem reduces to finding a linear combination $\boldsymbol{x}^{\boldsymbol{T}} \boldsymbol{a}$ of the rescaled variables which maximizes the ratio

$$
\frac{\boldsymbol{a}^{T} V \Lambda^{2} V^{T} \boldsymbol{a}}{\boldsymbol{a}^{T} V\left[I-\Lambda^{2}\right] V^{T} \boldsymbol{a}}
$$

Let $\boldsymbol{b}=V^{T} \boldsymbol{a}$. The above ratio is $\sum \lambda_{i}^{2} b_{i}^{2} / \sum\left(1-\lambda_{i}^{2}\right) b_{i}^{2}$, which is maximized by making only $b_{1}$ non-zero. Thus on the original variables, $\boldsymbol{a}$ is proportional to the first column of $S V$. 
The linear combination found by this process is called the first linear discriminant or the first canonical variate. Subsequent columns of $S V$ give further linear discriminants which are orthogonal to the previous ones and maximally separate the group means. Figure 44 illustrates this idea for two independent variables and three groups.

The group means differ only in the first $r$ variables. The quantity $(n-g) \lambda_{i}^{2} /(g-1)\left(1-\lambda_{i}^{2}\right)$ measures the ratios of between- to within-group variances on the $i^{\text {th }}$ canonical variate.

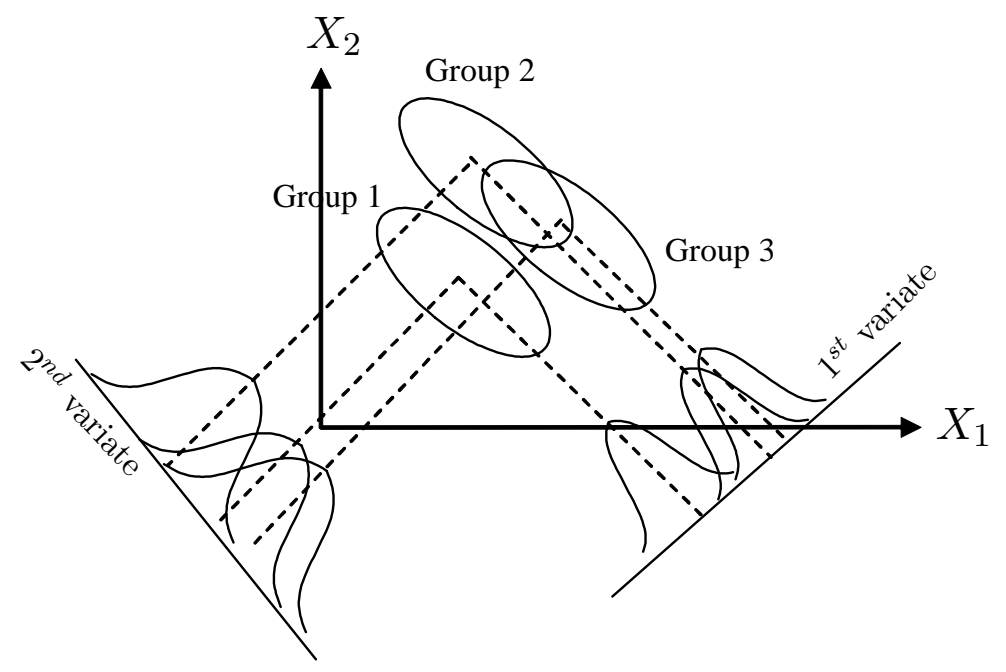

Figure 44: Fisher's idea for three-group discriminant analysis

\subsubsection{Classification rule}

The classification rule used here is based on the maximum likelihood principle that states: Assign sample $u$ to group $g$ if the likelihood of the observation vector $X_{u}$ is greater for group $g$ than for any other group [53]. There are several ways of applying the maximum likelihood principle. The approach taken here is to consider the posterior probability, $P\left(g \mid X_{u}\right)$, which is the most commonly used approach. It is called "posterior" in the sense that this is the probability of group membership conditioned on knowing $X_{u}$, that is after $p$ values of $X$ are obtained. Thus, according to the maximum likelihood principle, it is reasonable to assign sample $u$ to the group for which $P\left(g^{\prime} \mid X_{u}\right), g^{\prime}=1, \ldots, g$, is maximum. Using Bayes rule, and assuming there are $g$ groups, the posterior probability is given by

$$
P\left(g^{\prime} \mid X_{u}\right)=\frac{\pi_{g} \cdot P\left(X_{u} \mid g^{\prime}\right)}{\sum_{g^{\prime}=1}^{g} \pi_{g} \cdot P\left(X_{u} \mid g^{\prime}\right)}
$$


where $\pi_{g^{\prime}}$ denotes the probability that a randomly selected sample belongs to group $g^{\prime}$. That is, $\pi_{g^{\prime}}$ is the prior probability of membership in group $g^{\prime}$, "prior" in the sense that this is a probability of group membership before $X_{u}$ is known.

Assume that the groups are multivariate normal distributed,

$$
\hat{f}\left(g \mid X_{u}\right)=\frac{1}{\sqrt{(2 \pi)^{p}\left|\Sigma_{g}\right|}} \exp \left[-\frac{1}{2}\left(X-\bar{X}_{g}\right)^{T} \Sigma_{g}^{-1}\left(X-\bar{X}_{g}\right)\right]
$$

where $\bar{X}_{g}$ is the $p \times 1$ vector of means for group $g$, and $\Sigma_{g}$ is its $p \times p$ covariance matrix. The quadratic form in (5.21), $\Delta_{u g}^{2}=\left(X_{u}-\bar{X}_{g}\right)^{T} \Sigma_{g}^{-1}\left(X_{u}-\bar{X}_{g}\right)$ is the Mahalanobis distance between sample $u$ and the centroid for group $g$.

Working with estimators, $q_{g}$, of the true prior probabilities, $\pi_{g}$, and assuming that the $k$ group covariance matrices are equal, that is

$$
\Sigma_{1}=\Sigma_{2}=\cdots=\Sigma_{k}=\Sigma
$$

and using Mahalanobis distance, (5.20) can be written as follows in the multivariate normal case

$$
P\left(g \mid X_{u}\right)=\frac{q_{g} \cdot \exp \left(\frac{-1}{2} \Delta_{u g}^{2}\right)}{\sum_{g^{\prime}=1}^{g} q_{g^{\prime}} \cdot \exp \left(\frac{-1}{2} \Delta_{u g^{\prime}}^{2}\right)}
$$

Maximizing $P\left(g \mid X_{u}\right)$ in (5.23) is equivalent to maximizing $q_{g} \cdot \exp \left(\frac{-1}{2} \Delta_{u g}^{2}\right)$. This, in turn, is equivalent to maximizing the natural logarithm of the product:

$$
\ln q_{g}-\frac{1}{2} \Delta_{u g}^{2}=\ln q_{g}-\frac{1}{2}\left(X_{u}-\bar{X}_{g}\right)^{T} \Sigma_{g}^{-1}\left(X_{u}-\bar{X}_{g}\right)
$$

For classification purposes, the term $-\frac{1}{2} X_{u}^{T} \Sigma^{-1} X_{u}$ can be ignored because, for a given sample $u$, this term is common for all $g$. Thus, maximizing (5.24) is equivalent to maximizing

$$
L_{u g}=\left[\bar{X}_{g}^{T} \Sigma^{-1}\right] X_{u}-\frac{1}{2} \bar{X}_{g}^{T} \Sigma^{-1} \bar{X}_{g}+\ln q_{g}
$$

The maximum likelihood rule for the p-variate normal, equal covariance matrices case may be expressed as: assign unit $u$ to group $g$ if $L_{u g}>L_{u g^{\prime}}$ for $g \neq g^{\prime}$, where $L_{u g}$ is as defined in (5.25).

The expression of $L_{u g}$ is linear in $X_{u}$ and hence is called a linear classification function (LCF). In this research, a new sample will be classified in one of the three groups NS, MS or SS, according to a linear classification rule. 


\subsubsection{Misclassification Cost}

When a classifier is used to make a prediction about class membership of a new sample, some types of error can be more costly than others. For instance, false negative detection of severe suction may carry heavier consequences for patients, than false positive detection of severe suction. One way of penalizing such wrong decisions is by incorporating a cost matrix. Following [54], the expected cost of misclassification $(E C M)$ is

$$
E C M=\sum_{i=1}^{g} q_{i}\left(\sum_{\substack{k=1 \\ k \neq i}} P(k \mid i) C(k \mid i)\right)
$$

where $q_{i}$ is the prior probability of class $i, i=1, \ldots, g ; P(k \mid i)$, for $k \neq i$, is the probability of classifying a sample to class $k$ when it actually belongs to class $i$ and $C(k \mid i)$ is the cost of such misclassification with $C(k \mid i)=0$ when $k=i$.

The prediction rule that minimizes the $E C M$ is to assign a sample vector $X_{u}$ to class $i$ where

$$
\sum_{\substack{i=1 \\ i \neq k}} q_{i} P\left(X_{u} \mid i\right) \cdot C(k \mid i)
$$

is a minimum.

For instance, for $i=3$, assign $u$ to population 1 if

$$
\left[q_{2} P\left(X_{u} \mid 2\right) \cdot C(1 \mid 2)+q_{3} P\left(X_{u} \mid 3\right) \cdot C(1 \mid 3)\right]
$$

is smaller than

$$
\left[q_{1} P\left(X_{u} \mid 1\right) \cdot C(2 \mid 1)+q_{3} P\left(X_{u} \mid 3\right) \cdot C(2 \mid 3)\right]
$$

and

$$
\left[q_{1} P\left(X_{u} \mid 1\right) \cdot C(3 \mid 1)+q_{2} P\left(X_{u} \mid 2\right) \cdot C(3 \mid 2)\right]
$$




\subsubsection{Methods}

According to [53], discriminant analysis can be used in two different aspects: predictive discriminant analysis and descriptive discriminant analysis.

In Predictive Discriminant Analysis (PDA), there are a group of predictors or explanatory variables along with one criterion variable, which usually is categorical and indicates group membership. The goal of PDA is to predict membership of an object (i.e. a sample) in one of the criteria groups. With this regard, the predictors are viewed as inputs and the categorical variable as the outcome. The main question addressed in PDA is how accurately group membership can be predicted. Techniques in PDA are closely related with multiple linear regression, in which a linear combination of predictors is used. However, in PDA there are as many linear combinations as the number of groups.

Because PDA is concerned with classification accuracy (hit-rate), it is also important to know whether its hit-rate is better than that obtained by chance. Usually, a confusion matrix is used to present a PDA result. Each column of the confusion matrix represents the instances in an estimated group, while each row represents the instances in an actual group. A confusion matrix easily permits to see if the classifier has mislabeled some instances. Table 5 shows an example of a confusion matrix for a two-group classifier.

Table 5: Example of a Confusion Matrix for a two-group classifier

\begin{tabular}{cc|cc} 
& & \multicolumn{2}{|c}{ PREDICTED } \\
& & Negative & Positive \\
\hline \multirow{2}{*}{ ACTUAL } & Negative & $a$ & $b$ \\
& Positive & $c$ & $d$ \\
\hline
\end{tabular}

From the confusion matrix, several important quantities can be defined. For instance:
a) The accuaracy (hit-rate) $(A C)$ is the proportion of the total number of predictions that were correctly classified

$$
A C=\frac{a+d}{a+b+c+d}
$$
b) The true positive rate $(T P)$ or Sensitivity is the pro- portion of positive cases that were correctly identified$$
T P=\frac{d}{c+d}
$$ 
c) The false positive rate $(F P)$ is the proportion of nega- $\quad F P=\frac{b}{a+b}$ tives cases that were incorrectly classified as positive

d) The true negative rate $(T N)$ or Specificity is the pro- $\quad T N=\frac{a}{a+b}$ portion of correctly classified negatives cases

e) The false negative rate $(F N)$ is the proportion of posi- $\quad F N=\frac{c}{c+d}$ tives cases that were incorrectly classified as negative

To verify how much improvement in classification accuracy is due to the discriminant model, we compare its performance with the proportional chance criteria. This decision rule classifies observations based on the relative frequency with which each group appears in the confusion matrix [53]. Table 6 illustrates the expected result by chance in the two-group problem case. Let $p$ denote the proportion of the entire sample consisting of group 1 observations. Then the proportional chance decision rule is as follows:

Assign observation to group 1 with probability $=p$

Assign observation to group 2 with probability $=(1-p)$

Table 6: Expected number classified by chance

\begin{tabular}{cc|ccc} 
& & \multicolumn{3}{|c}{ PREDICTED } \\
& & Group 1 & Group 2 & Total \\
\hline & Group 1 & $n p^{2}$ & $n p(1-p)$ & $n p$ \\
ACTUAL & Group 2 & $n p(1-p)$ & $n(1-p)^{2}$ & $n(1-p)$ \\
& Total & $n p$ & $n(1-p)$ & $n$ \\
\hline
\end{tabular}

The expected number of hits in the proportional chance criteria is $h=n p^{2}+n(1-p)^{2}$. In addition, the standard deviation of the proportional chance criteria is $\sigma_{\text {cpro }}=\sqrt{n h(1-h)}$ [53]. Using $\sigma_{\text {cpro }}$, it is possible to perform a $t$-test to compare the classification performances of the discriminant model versus the proportional chance criteria. 
In Descriptive Discriminant Analysis (DDA) the categorical variable plays the role of explanatory variable and the predictors are the outcome variables. In other words, in DDA the model is reversed as compared with the PDA approach and one is concerned with group separability rather than prediction accuracy when performing DDA. Techniques in DDA are closely related to MANOVA. Indeed, linear combinations of outcome variables are commonly used in DDA to interpret, in some cases, the resulting variable combinations that are associated with group differences.

Another difference between PDA and DDA is that in the former there are as many linear classification functions (LCFs) as there are groups. In DDA, the number of linear discriminant functions (LDFs) coincides with the rank of the matrix $\boldsymbol{W}^{-1} \boldsymbol{B}, r=\min (p, g-1)$. To interpret the results from DDA, information from these LDFs are commonly used, specifically, the standardized discriminant function coefficients and discriminant loadings. Some authors [52] believe that the standardized discriminant function coefficients may carry information about group separability. However, most researchers look at the discriminant loadings that underlie group differences [53]. The discriminant loadings are correlations between the predictors and the discriminant scores.

As stated earlier, $r=\min (p, g-1)$ LDFs may be extracted. The question is then how many of them should be considered to interpret group differences. One way to answer this question is by means of a proportion-of-variance approach. The $j^{\text {th }}$ eigenvalue, $\lambda_{j}$, of $\boldsymbol{W}^{-1} \boldsymbol{B}$ represents the ratio of the between-group to within-group variability with respect to scores in the $j^{\text {th }} \mathrm{LDF}$. That is, $\lambda_{j}$ reflects a proportion of variance in a $p$-variable system accounted for by the $j^{\text {th }}$ LDF. In addition, multiple discriminant analysis (MDA) can be seen as a special case of canonical correlation. In fact, the eigenvalues from MDA are related to the eigenvalues from canonical correlation (CC) as [52]

$$
\lambda_{j}^{M D A}=\frac{\lambda_{j}^{C C}}{1-\lambda_{j}^{C C}}
$$

Thus, it is possible to test the significance of the model using the following version of Wilks' $\Lambda$ statistic:

$$
\Lambda=\sum_{j} \frac{1}{1+\lambda_{j}^{M D A}}
$$


The Wilks' $\Lambda$ is used to test the significance of a discriminant function. A significant $\Lambda$ means that the null hypothesis that the groups have the same mean on the discriminant function scores can be rejected. The smaller the lambda, $\Lambda$, the greater the group means differences. A Bartlett's chi-square test statistic for $\Lambda$ is

$$
V=\left[(n-1)-\frac{p+g}{2}\right] \sum_{j} \ln \left(1+\lambda_{j}^{M D A}\right)
$$

with $p(g-1)$ degrees of freedom, where $p$ is the number predictors, and $g$ is the number of groups.

To present the DDA results a table is usually used, containing the following information: the eigenvalues of the $\boldsymbol{W}^{-1} \boldsymbol{B}$ matrix, the explained variance attributed to each eigenvalue, the standardized discriminant function coefficients, the discriminant loadings and the group means on the discriminant functions.

Finally, a plot of the samples in the LDF space (usually only the first two dimensions are considered) can be used to determine the number of LDFs to retain for interpretation of group separation.

\subsection{EXPERIMENTAL RESULTS}

This section describes how experimental data were used to design and test the suction detection system. These data were analyzed off-line. The pump flow signal and other hemodynamic variables were recorded in an in-vivo study performed on a calf. This study was conducted in association with LaunchPoint, LLC (Goleta, CA) and WorldHeart, Inc. The WorldHeart LVAD was used in this experiment and data were sampled at a rate of $500 \mathrm{~Hz}$. The detection system and the discriminant analysis of the data were implemented using MATLAB ${ }^{6}$. The in-vivo study had two main goals: to test several control approaches, and to assess suction indices performance. Suction was induced either by overpumping or by clamping the vena cava. The first test method consists of an increase in pump speed, using a ramp profile. Following the test protocol, the speed ramp was applied before any drug

\footnotetext{
${ }^{6}$ The MathWorks Inc., Natick, MA
} 
administration to the animal, and data recorded in this condition were used as base-line. The second test method, vena cava occlusion, causes less blood to return to the animal's heart. Consequently, less blood is available for the pump to draw, and suction occurs. In this particular in-vivo experiment, administration of Norepinephrine (a vaso constrictor) was used to change systemic vascular resistance (SVR). The researchers wanted to know how SVR changes would affect pump flow. In addition, at the end of the experiment, Esmolol (a cardioselective drug that affects heart contractility) was administrated to the animal to verify how changes in the contractility of the heart impacts pump flow and the occurrence of suction.

\subsubsection{Data description}

A total of 35 data files were recorded. The data were classified by a human expert into three groups, according to the pump status previously defined in Section 5.1: No Suction (NS), Moderate Suction (MS), and Severe Suction (SS). This classification procedure was based on the analysis of pump flow (PF), pump speed (PS), left ventricular pressure (LVP) and pump inlet pressure (PIP), using a window 5 heart beats long. Each one of these windows was classified by the expert, resulting in a data base with 1,197 samples of the pump flow signal. Figure 45 illustrates the steps taken in our approach. Table 7 shows the data statistics, means and standard deviations of each feature variable for each type of suction. Most of the samples 647 (54\%) belongs to group NS, whereas groups MS and SS present 429 (36\%) and $121(10 \%)$, respectively, of the data.

Looking at the group means for each feature, we noticed that these means are not equal across groups. This fact is even more evident by looking at the box-plots for each feature, shown in Figure 46. These plots also reveal that some indices have skewed distributions. Having normal distributed features is desirable, but not essential for applying the DA method [53].

The best way to test the hypothesis of equal group means is by performing a multivariate analysis of variance, MANOVA. A one way MANOVA was performed using the $\mathrm{SAS}^{7}$ package.

\footnotetext{
${ }^{7}$ SAS Institute Inc., Cary, NC, USA
} 
Table 8 presents these results. They show that the difference between groups is significant at $p<0.0001$, based on a Wilks's $\Lambda=0.157$. The MANOVA result also shows the partial correlation matrix associated with the Within group covariance matrix. In our data base, none of the indices are very strongly correlated; the strongest correlation $(r=0.57)$ is between $S I_{3}$ and $S I_{6}$.

The Mahalanobis distance between group centroids shows that NS samples are more "similar" to MS, since the Mahalanobis distance between their means is only 3.17, whereas the distances from these two group means to the SS group centroid are 13.61 and 10.47, respectively. This result is in agreement with our previous analysis of inflow resistance, presented in section 5.1. We had concluded that pump states NS and MS present similar physiological characteristics.

Since we have $p=8$ predictors and $g=3$ groups, we should expect for the group means to lie in a two dimensional space (not necessarily aligned), $\min (p, g-1)=2$. This is actually the case, and the MANOVA result (Table 8, IV) confirms this hypothesis.

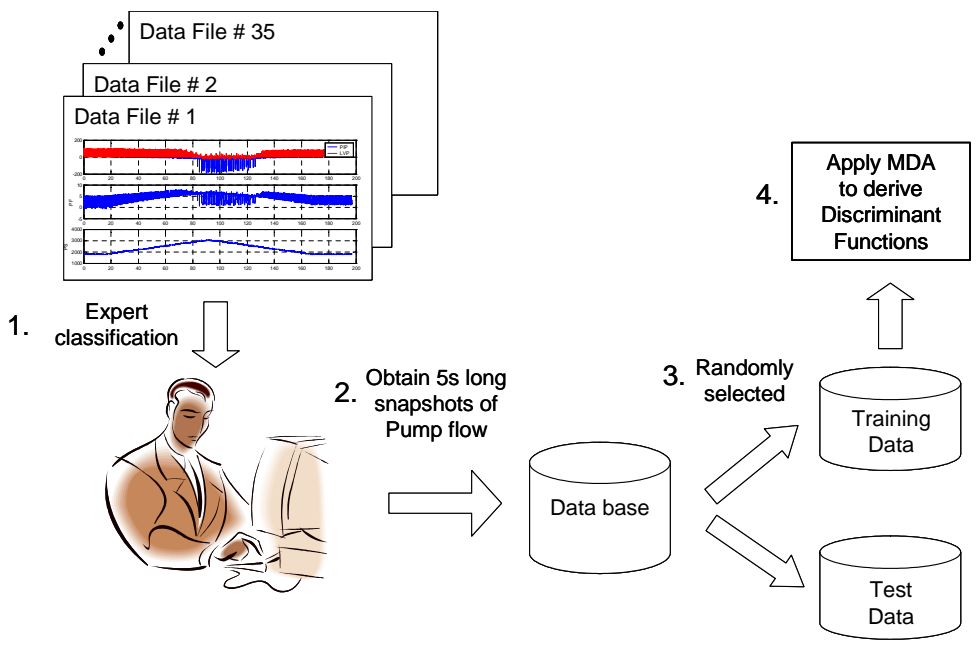

Figure 45: Detector design steps 
Table 7: Data Statistics

\begin{tabular}{|c|c|c|c|c|c|c|c|c|}
\hline \multirow[b]{2}{*}{ Feature } & \multicolumn{2}{|c|}{$\begin{array}{c}\text { NS } \\
n_{1}=647 \\
(54 \%)\end{array}$} & \multicolumn{2}{|c|}{$\begin{array}{c}\text { MS } \\
\left(n_{2}=429\right) \\
(36 \%)\end{array}$} & \multicolumn{2}{|c|}{$\begin{array}{c}\mathbf{S S} \\
\left(n_{3}=121\right) \\
(10 \%)\end{array}$} & \multicolumn{2}{|c|}{$\begin{array}{c}\text { Overall } \\
(N=1197) \\
(100 \%)\end{array}$} \\
\hline & Mean & S.D. & Mean & S.D. & Mean & S.D. & Mean & S.D. \\
\hline$S I_{1}$ & 1.747 & 0.453 & 1.239 & 0.265 & 0.655 & 0.299 & 1.455 & 0.522 \\
\hline$S I_{2}$ & 0.074 & 0.053 & 0.145 & 0.108 & 0.658 & 0.561 & 0.158 & 0.258 \\
\hline$S_{3}$ & 0.413 & 0.047 & 0.361 & 0.040 & 0.610 & 0.145 & 0.414 & 0.094 \\
\hline$S I_{4}$ & 0.050 & 0.035 & 0.130 & 0.078 & 0.193 & 0.095 & 0.093 & 0.079 \\
\hline$S I_{5}$ & 0.052 & 0.035 & 0.069 & 0.049 & 0.375 & 0.224 & 0.091 & 0.126 \\
\hline$S I_{6}$ & 7.133 & 2.029 & 5.057 & 0.640 & 6.152 & 2.226 & 6.290 & 1.949 \\
\hline$S I_{7}$ & -7.774 & 0.795 & -7.966 & 0.787 & -9.835 & 2.473 & -8.051 & 1.242 \\
\hline$S I_{8}$ & 0.018 & 0.016 & 0.037 & 0.033 & 0.178 & 0.112 & 0.041 & 0.063 \\
\hline
\end{tabular}



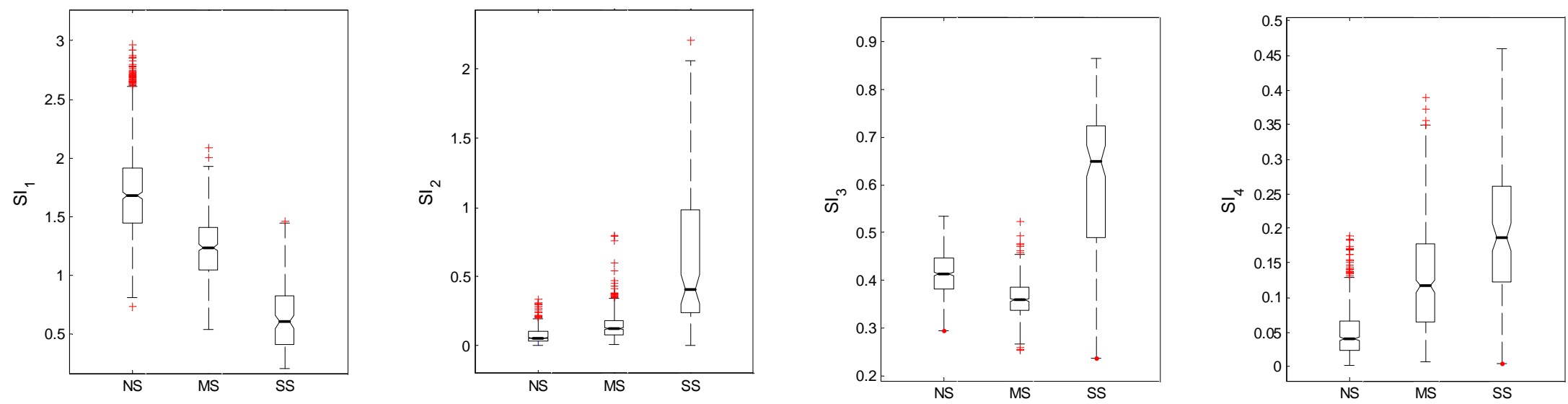

ว้
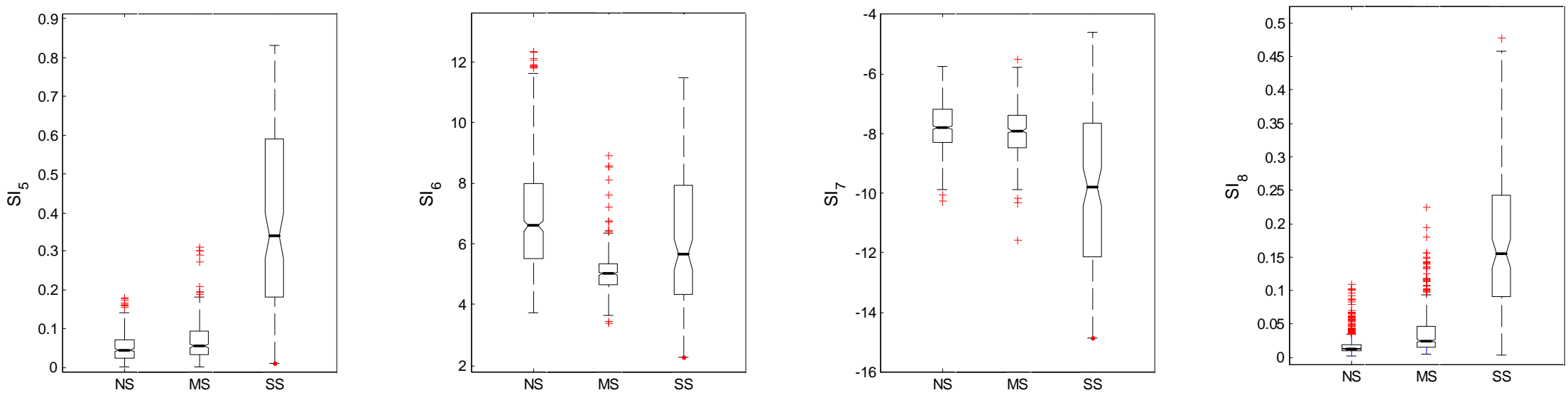

Figure 46: Box plots of the features per group 
Table 8: MANOVA Results for groups

I. Multivariate Statistics:

\begin{tabular}{lrcrrc}
\hline Statistic & Value & F & Num df & Den df & Pr $>$ F \\
\hline Wilks's $\Lambda$ & 0.157 & 225.70 & 16 & 2374 & $<0.0001$ \\
Pillai's Trace & 1.129 & 192.58 & 16 & 2376 & $<0.0001$ \\
Hotelling-Lawley Trace & 3.535 & 262.05 & 16 & 1938.8 & $<0.0001$ \\
Roy's Greatest Root & 2.908 & 431.89 & 8 & 1188 & $<0.0001$ \\
\hline
\end{tabular}

II. Correlation Coefficients from the Within group covariance matrix

\begin{tabular}{lrccccccc}
\hline & $S I_{1}$ & $S I_{2}$ & $S I_{3}$ & $S I_{4}$ & $S I_{5}$ & $S I_{6}$ & $S I_{7}$ & $S I_{8}$ \\
$S I_{1}$ & 1.00 & & & & & & & \\
$S I_{2}$ & -0.16 & 1.00 & & & & & & \\
$S I_{3}$ & 0.26 & 0.28 & 1.00 & & & & & \\
$S I_{4}$ & -0.24 & 0.22 & -0.21 & 1.00 & & & & \\
$S I_{5}$ & -0.19 & 0.30 & 0.50 & 0.16 & 1.00 & & & \\
$S I_{6}$ & 0.56 & 0.16 & 0.57 & -0.11 & 0.01 & 1.00 & & \\
$S I_{7}$ & 0.14 & -0.25 & -0.35 & 0.22 & -0.14 & -0.21 & 1.00 & \\
$S I_{8}$ & -0.24 & 0.32 & 0.36 & 0.21 & 0.46 & 0.07 & -0.26 & 1.00 \\
\hline
\end{tabular}

III. Pairwise Mahalanobis distance between group means

\begin{tabular}{rrrr}
\hline & NS & MS & SS \\
NS & 0 & & \\
MS & 3.17 & 0 & \\
SS & 13.61 & 10.47 & 0 \\
\hline
\end{tabular}

IV. Dimensionality test of group means

\begin{tabular}{cc}
\hline Space dimension & $\mathrm{P}$ value \\
2 & $<0.0001$ \\
\hline
\end{tabular}




\subsubsection{Minimum number of samples}

A "rule of thumb" for the minimum sample size in discriminant analysis is that the smallest group be comprised of at least $3 * p$ samples, where $p$ is the number of predictors [53]. In our case, the number of samples in each group was equal, so that, in the training phase, all groups were equally likely. This compensates for the reduced number of severe suction samples in the data base. Since we have 8 predictors (indices), a minimum of 24 samples for each one of the groups would be needed, given a total of $N=72$ samples in the training set. A quick simulation study was performed to determine the number of samples per group, $n$, to use in the test set. Starting with only $n=10$ samples per group (thus, a total training set of 30 samples), we derived the discriminant model and evaluated its hit-rate using the leave-one-out method. The number of samples was then increased by 10 until 50 samples from each group were present in the training set. Figure 47 shows this simulation result.

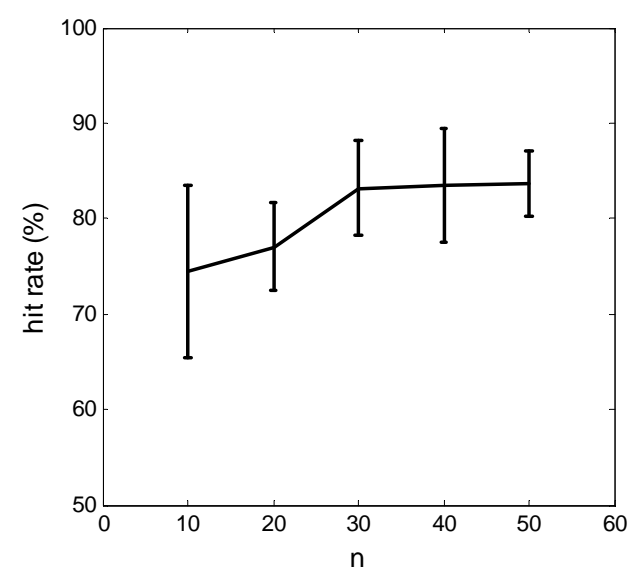

(a) Two-group classification problem

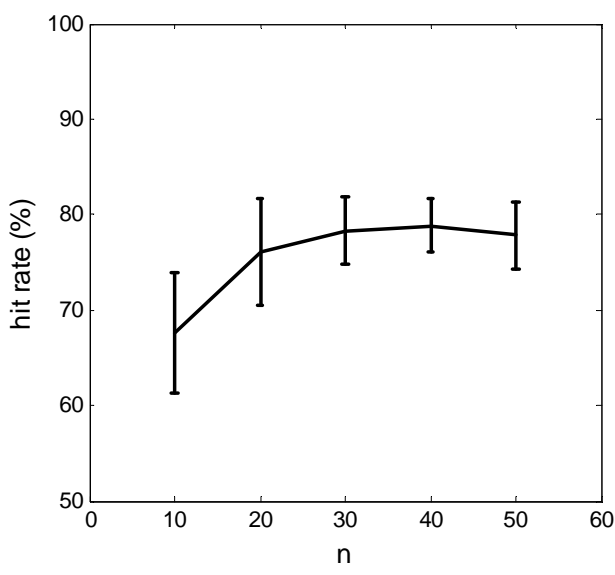

(b) Three-group classification problem

Figure 47: Hit-rate versus number of samples per group

As the number of samples is increased, so does the hit-rate. However, there is no significant improvement in accuracy, for $n \geq 30$, which is a value close to the "rule of thumb" number, 24, given in [53].

In the next section, the methods described in Section 5.2.4 are applied to experimental data. Two classification problems are considered: two and three-group classification. Although our expert had identified three possible pumping states, the use of two states 
(Suction/ No Suction) is a common approach adopted in the literature [65, 66]. The twogroup classification will be studied for completeness purposes. PDA and DDA methods will be used for the training set in both problems (two and three group classification), whereas only PDA will be used in the test set.

\subsubsection{The two-group classification problem}

When only two groups are considered, the classifier has to label a given sample either as No Suction, N or Suction, S. Due to their similarities from a physiologic stand point, group N contains both groups NS and MS, whereas group S is the same as group SS.

To design the linear classifier, 30 samples were randomly drawn from each one of the groups $\mathrm{N}$ and $\mathrm{S}$. This was done to ensure that sufficient information on suction groups was obtained. In this case, our main goal is to classify the remaining 1,127 samples either as No Suction (N) or Suction (S).

5.3.3.1 Descriptive Discriminant Analysis Table 9 presents the DDA results. Since this is a dichotomous problem, only one linear discriminant function is needed. The eigenvalue, $\lambda=5.376$, of the LDF accounts for $100 \%$ of the differences in the group means. Indices $S I_{1}, S I_{3}$ and $S I_{5}$ present the highest correlation values, -0.88, 0.82 and 0.78 respectively, showing that these predictors play a major role in distinguishing between suction and nosuction patterns. This suggests that pump flow samples in the S set present augmented harmonic energy (related to the fundamental) and their time wave form is asymmetric.

\subsubsection{Predictive Discriminant Analysis Table 10 shows the confusion matrix for} the training data. The results are good. Fifty eight samples were correctly classified (i.e, 30 of $30 \mathrm{~N}$ samples were correctly identified as $\mathrm{N}$ and 28 of 30 suction cases were correctly identified as S), giving an overall hit-rate of $96 \%$.

Because the group sizes were equal, the expected number of hits given by the proportional chance criteria is $n / 2$ and the hit-rate is $50 \%$. Since the discriminant approach gave a hit-rate of $96 \%$, it performed better than the proportional chance criteria. The standard 
Table 9: DDA result for the two-group classification problem

\begin{tabular}{|c|c|c|}
\hline Eigenvalue & Difference $\operatorname{Pr}$ & ction Cumulative \\
\hline \multicolumn{2}{|l|}{5.376} & 1.00 \\
\hline Feature & $\begin{array}{c}\text { Standardized } \\
\text { Discriminant } \\
\text { Coefficients }\end{array}$ & $\begin{array}{c}\text { Discriminant } \\
\text { loadings } \\
\text { (correlations) }\end{array}$ \\
\hline$S I_{1}$ & -1.64 & -0.88 \\
\hline$S I_{2}$ & 0.86 & 0.64 \\
\hline$S I_{3}$ & 16.84 & 0.82 \\
\hline$S I_{4}$ & 4.68 & 0.72 \\
\hline$S I_{5}$ & -2.16 & 0.78 \\
\hline$S I_{6}$ & -0.46 & -0.06 \\
\hline$S I_{7}$ & 0.33 & -0.41 \\
\hline$S I_{8}$ & -2.32 & 0.70 \\
\hline
\end{tabular}

\section{Group means on LDF space}

\begin{tabular}{lr}
\hline Group & $\alpha_{1}$ \\
$\mathrm{~N}$ & -2.27 \\
$\mathrm{~S}$ & 2.27 \\
\hline
\end{tabular}

deviation of the proportional chance criteria is $\sigma_{\text {cpro }}=3.87$; and a $t$-statistic to compare the classification performances of the discriminant model versus the proportional chance criteria is $t=\frac{58-30}{3.87}=7.22$ with $\mathrm{df}=59$. Clearly, the result is significant, showing that information about pump flow features improves our ability to discriminate the pump status. Figure 48 shows that the distribution of discriminant scores for $\mathrm{N}$ samples is left-shifted, which implies that most of the discriminant scores are negative, while S samples are more likely to have positive discriminant scores (right-shifted distribution). This fact is in agreement with the 
Table 10: Confusion Matrix for the classifier ${ }^{a}$

\begin{tabular}{cc|ccc} 
& & \multicolumn{3}{|c}{ PREDICTED } \\
& & $\mathrm{N}$ & $\mathrm{S}$ & Total \\
\hline \multirow{2}{*}{ ATUAL } & $\mathrm{N}$ & 30 & 0 & 30 \\
& & & $(100 \%)$ & $(100 \%)$ \\
& $\mathrm{S}$ & 2 & 28 & 30 \\
& & $(7 \%)$ & $(93 \%)$ & $(100 \%)$ \\
\hline
\end{tabular}

${ }^{a} \mathrm{~N}=$ No Suction, $\mathrm{S}=$ Suction

location of the projected group means in the discriminant space: -2.27 for group $\mathrm{N}$ and 2.27 for group S.

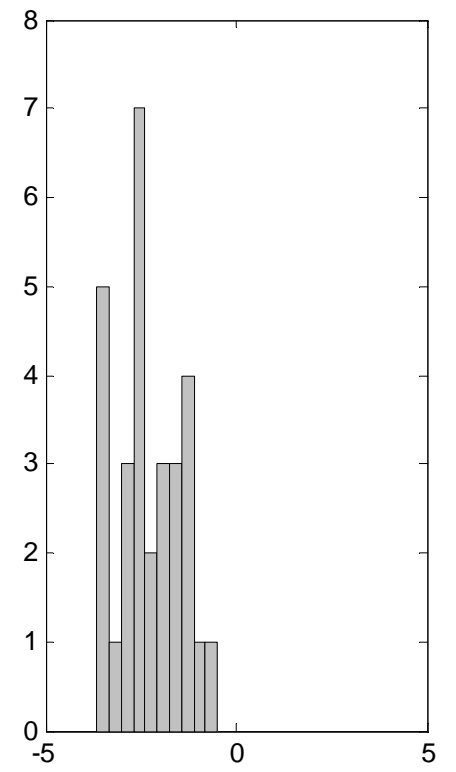

(a) No Suction

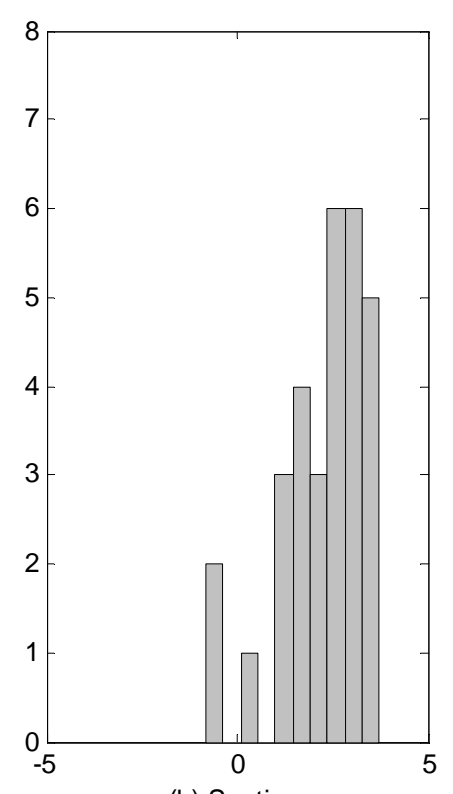

(b) Suction

Figure 48: Histograms of discriminant scores for training set 


\subsubsection{Classifying the test set for the two-group problem}

The discriminant model obtained from the training data set was used to classify the remaining 1,137 samples in the data base. Table 11 shows the confusion matrix in this case. The model was able to identify correctly 1,089 samples, giving a hit-rate of $95 \%$. To verify how the discriminant model compares with the proportional chance criteria, notice that now $p=1046 / 1137=0.92$. Therefore, the expected number of correct classifications by chance is $1137\left[0.92^{2}+(1-0.92)^{2}\right]=969$, which gives a hit-rate of $969 / 1137=85 \%$. The variance of the proportional chance criteria is $\sigma_{\text {cpro }}=11.96$, and $t=\frac{1089-969}{11.96}=10.02(\mathrm{df}=1,136)$, which is significant. Both the false positive (3\%) and false negative (14\%) rates increased compared to those in the training set (see Table 10). This was expected, since hit-rates from training sets tend to be positively biased. Figure 49 shows the distributions of the discriminant scores for both groups $\mathrm{N}$ and $\mathrm{S}$.

Table 11: Confusion Matrix for test set ${ }^{a}$

\begin{tabular}{|c|c|c|c|c|}
\hline & \multicolumn{2}{|c|}{ PREDICTED } & \multirow[b]{2}{*}{ Total } \\
\hline & & $\mathrm{N}$ & S & \\
\hline \multirow{4}{*}{ ATUAL } & $\mathrm{N}$ & 1011 & 35 & 1046 \\
\hline & & $(97 \%)$ & $(3 \%)$ & $(100 \%)$ \\
\hline & \multirow[t]{2}{*}{$\mathrm{S}$} & 13 & 78 & 91 \\
\hline & & $(14 \%)$ & $(86 \%)$ & $(100 \%)$ \\
\hline
\end{tabular}

\subsubsection{The three-group classification problem}

When more than two groups are considered, the discriminant problem becomes a Multiple Discriminant Analysis (MDA) problem. Indeed, at least $(g-1)$ discriminant functions are needed in order to distinguish between $g$ groups, since $g$ is less than the number of predictors. A classifier was designed using a total of 90 samples, 30 from each group. The goal in this 

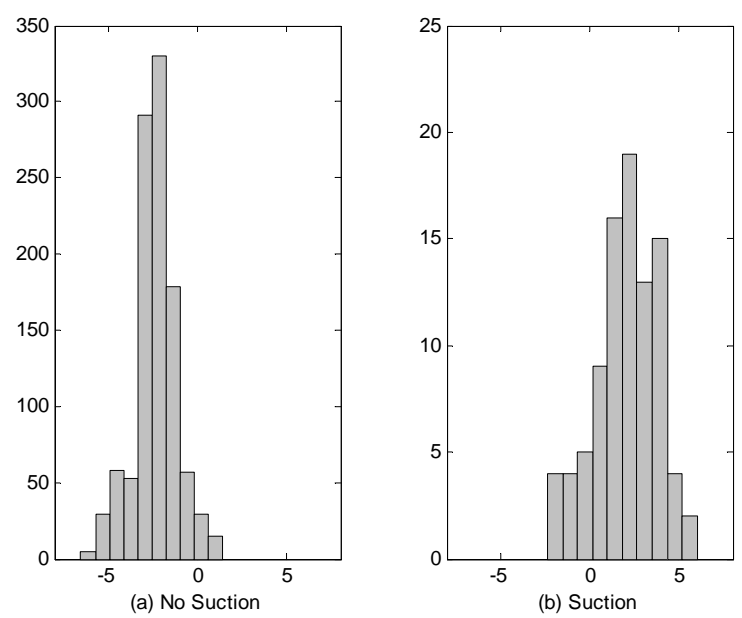

Figure 49: Histograms of discriminant scores for test set

case is to classify the remaining 1,107 samples as No Suction (NS), Moderate Suction (MS) or Severe Suction (SS).

5.3.5.1 Descriptive Discriminant Analysis Table 12 presents these results. The first discriminant function, $\alpha_{1}$, explains most of the differences between the groups in the data, since its proportion was 0.8719 . The second discriminant function, $\alpha_{2}$, accounts for only 0.1281 of the difference. The columns labeled $C_{1}, C_{2}$ show the discriminant loadings. Indices $S I_{3}=0.85, S I_{8}=0.84, S I_{1}=-0.80$ and $S I_{5}=-0.80$ in column $C_{1}$ correlated most strongly with the first discriminant function. As for the second discriminant function, correlations are small as compared with $\alpha_{1}$. Indeed, $S I_{6}$ has correlation $r=-0.71$ with the second discriminant function, $\alpha_{2}$.

Looking at means in the first discriminant, the mean for SS is 2.62; for NS and MS, the means are -1.63 and -0.99 , respectively. In addition, we know that the first discriminant function is mainly correlated with $S I_{3}, S I_{1}$ and $S I_{8}$. This suggests that SS is different from MS and NS because pump flow samples in SS are asymmetric, present augmented harmonic energy (related to the fundamental frequency band) and higher spread of energy in the timefrequency plane. Hence, the first discriminant function separates SS from the other two groups. 
Table 12: DDA result for the three-group classification problem

\begin{tabular}{rrrr}
\multicolumn{4}{l}{ Eigenvalues of $W^{-1} B$} \\
\hline Eigenvalue & Difference & Proportion & Cumulative \\
3.6333 & 3.0994 & 0.8719 & 0.8719 \\
0.5339 & & 0.1281 & 1.0000
\end{tabular}

\begin{tabular}{lccccc}
\hline Feature & \multicolumn{2}{c}{$\begin{array}{c}\text { Standardized } \\
\text { Discriminant } \\
\text { Coefficients }\end{array}$} & & \multicolumn{2}{c}{$\begin{array}{c}\text { Discriminant } \\
\text { loadings } \\
\text { (correlations) }\end{array}$} \\
\cline { 6 - 7 }$S I_{1}$ & -0.96 & 1.59 & & $\mathbf{- 0 . 8 0}$ & 0.45 \\
$S I_{2}$ & 0.79 & 0.07 & & 0.69 & 0.06 \\
$S I_{3}$ & 12.91 & 10.53 & & $\mathbf{0 . 8 5}$ & 0.44 \\
$S I_{4}$ & 3.69 & -4.07 & & 0.67 & -0.43 \\
$S I_{5}$ & -1.98 & -1.35 & & 0.83 & 0.12 \\
$S I_{6}$ & -0.27 & 0.11 & & -0.04 & $\mathbf{- 0 . 7 1}$ \\
$S I_{7}$ & 0.22 & 0.18 & & -0.54 & -0.12 \\
$S I_{8}$ & 4.10 & 1.03 & & $\mathbf{0 . 8 4}$ & 0.07 \\
\hline
\end{tabular}

Group means on LDF space

\begin{tabular}{lrr}
\hline Group & $\alpha_{1}$ & $\alpha_{2}$ \\
NS & -1.63 & 0.80 \\
MS & -0.99 & -0.94 \\
SS & 2.62 & 0.14 \\
\hline
\end{tabular}

The second discriminant score separates group MS from the other two. MS samples have projected mean -0.94 in the second dimension, whereas NS and SS have means 0.80 and 0.14 respectively. Even though that difference in group means in the second discriminant score is not as pronounced as it is in the first one, the second discriminant function plays an important role in separating MS samples from the others. Figure 50 shows a plot of the 
training data in the discriminant space. Using Wilks' $\Lambda$ statistic to test the null hypothesis that there is no difference across NS, MS and SS, we have $n=90, p=8, g=3, \lambda_{1}^{M D A}=3.633$, and $\lambda_{2}^{M D A}=0.533$. Substituting these values into equation (5.28) gives

$$
V=[90-11 / 2][\ln (4.633)+\ln (1.533)]=163.75
$$

With 16 degrees of freedom, this result is significant at $p<0.01$.

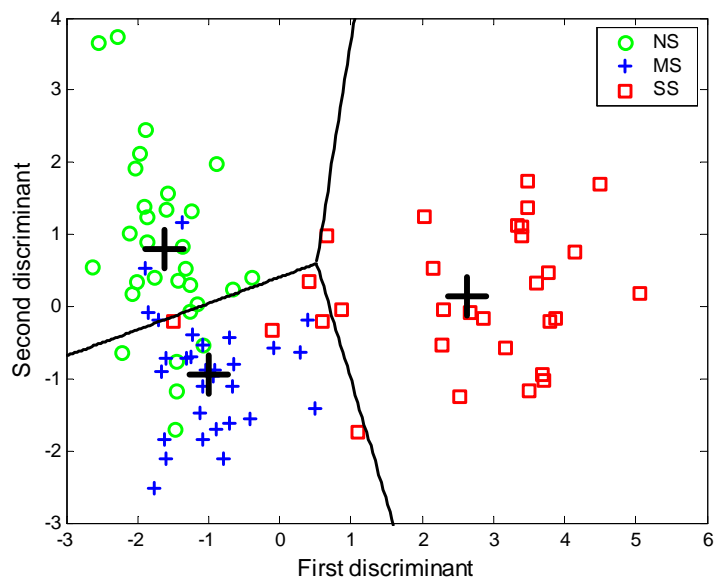

Figure 50: Plot of training data in discriminant function space

5.3.5.2 Predictive Discriminant Analysis Table 13 presents the confusion matrix on the training set.

The discriminant model presents a good result compared to the proportional chance criteria, which is given by $n\left(p_{1}^{2}+p_{2}^{2}+p_{3}^{2}\right)$. In this case, $p_{i}=1 / 3, i=1,2,3$, yielding a chance hit-rate of $33 \%$. This is much less than the $83 \%$ obtained with the discriminant model. In addition, no SS samples were mislabeled as NS. Even though $17 \%$ of SS samples were mislabeled as MS, they still were recognized as suction. Moreover, no NS samples were mislabeled as SS, and misclassification of NS as MS is not as critical as identifying NS as SS. 
Table 13: Confusion Matrix for training set

\begin{tabular}{|c|c|c|c|c|c|}
\hline & \multicolumn{3}{|c|}{ PREDICTED } & \multirow[b]{2}{*}{ Total } \\
\hline & & NS & MS & $\mathrm{SS}$ & \\
\hline \multirow{6}{*}{ ACTUAL } & NS & 24 & 6 & 0 & 30 \\
\hline & & $(80 \%)$ & $(20 \%)$ & $(0 \%)$ & $(100 \%)$ \\
\hline & MS & 4 & 26 & 0 & 30 \\
\hline & & $(13 \%)$ & $(87 \%)$ & $(0 \%)$ & $(100 \%)$ \\
\hline & \multirow[t]{2}{*}{ SS } & 0 & 5 & 25 & 30 \\
\hline & & $(0 \%)$ & $(17 \%)$ & $(83 \%)$ & $(100 \%)$ \\
\hline
\end{tabular}

\subsubsection{Classifying the test set for the three-group problem}

The designed DA model was applied to the test set. In this case, our goal was to classify the remaining samples $(1,107)$ into one of the three groups. Table 14 shows the confusion matrix in this case. It reveals that $81 \%$ of the severe suction cases were correctly classified by the model, and none of them was identified as NS. If we had to rely on chance alone, the hit-rate would have been $45 \%$, because $p_{1}=617 / 1107, p_{2}=399 / 1107$, and $p_{3}=91 / 1107$ in this case.

Even though the overall hit-rate (accuracy) of $81 \%$ is less than that observed in the two-group problem (95\%), the false positive rate in the severe suction group decreases considerably. This implies that our ability to identify severe suction cases was improved in the three-group classification problem. This is in agreement with the expert's opinion, who considers that moderate suction is not as deleterious for the patient's health as is severe suction. Therefore, the multidiscriminant analysis-based classifier will be adopted in this research study. Figure 51 shows a plot of the test data in the discriminant space. Note that SS cases are well separated from the others groups in the first discriminant score. This result is consistent with that obtained for the training set (see Figure 50). 
Table 14: Confusion Matrix for test set ${ }^{a}$

\begin{tabular}{|c|c|c|c|c|c|}
\hline & \multicolumn{3}{|c|}{ PREDICTED } & \multirow[b]{2}{*}{ Total } \\
\hline & & NS & MS & SS & \\
\hline \multirow{6}{*}{ ACTUAL } & NS & 516 & 101 & 0 & 617 \\
\hline & & $(83.6 \%)$ & $(16.4 \%)$ & $(0 \%)$ & $(100 \%)$ \\
\hline & MS & 79 & 310 & 10 & 399 \\
\hline & & $(19.8 \%)$ & $(77.6 \%)$ & $(2.6 \%)$ & $(100 \%)$ \\
\hline & \multirow[t]{2}{*}{ SS } & 0 & 17 & 74 & 91 \\
\hline & & $(0 \%)$ & $(18.6 \%)$ & $(81.4 \%)$ & $(100 \%)$ \\
\hline
\end{tabular}

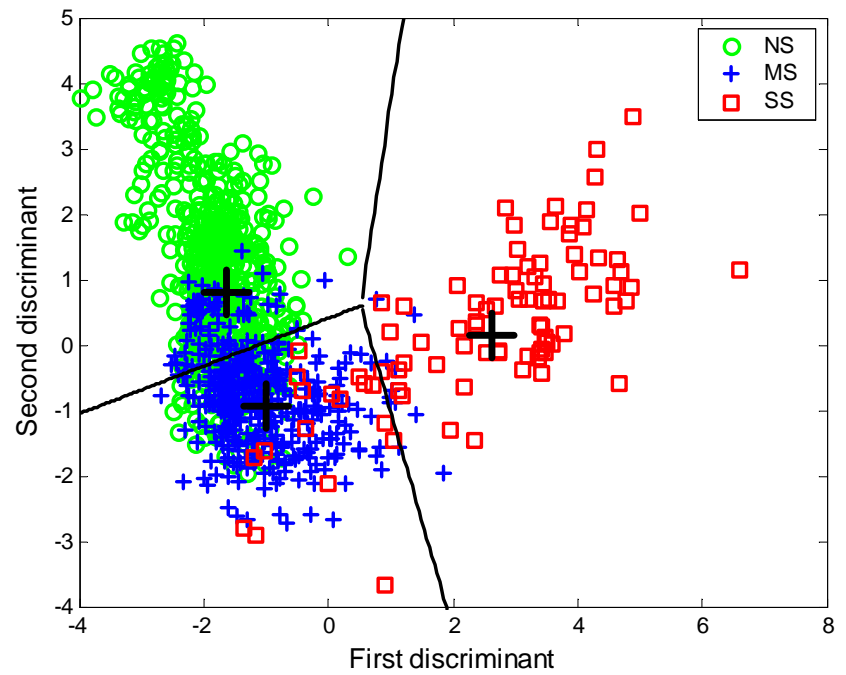

Figure 51: Plot of test data in discriminant function space 


\subsection{SIMULATION STUDIES}

Simulation studies were carried out to evaluate the suction detection performance in response to physiological parameter changes and robustness to noise. To this end, the cardiovascularpump model described in Section 4.3 was used to generate the input for the suction detector, i.e., the pump flow signal. Figure 52 illustrates a block diagram interconnection between the cardiovascular-pump model and the suction detector where $\omega$ is a pre-defined pump speed

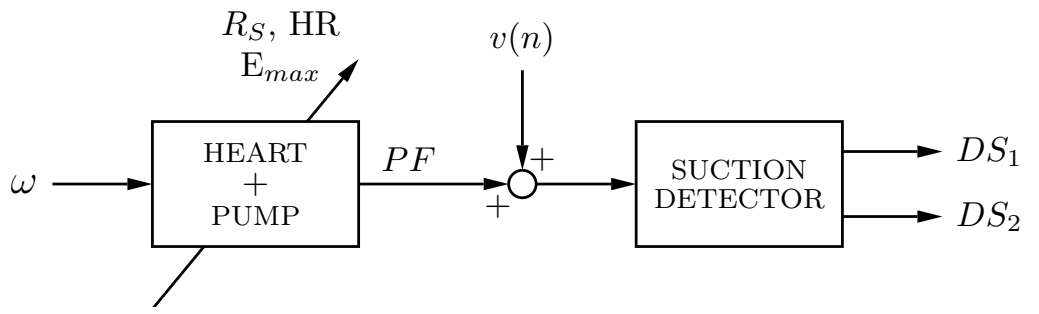

Figure 52: Block diagram of cardiovascular-pump model and suction detection

profile, $R_{S}$ represents the patient's systemic vascular resistance (SVR) and was used to change preload; $E_{\max }$ is an elastance parameter (see equation 4.3) used to change contractility. $P F$ is pump flow and $D S_{1}$ and $D S_{2}$ are the two discriminant scores. Signal $v(n)$ is a white gaussian noise added to the pump flow signal. It represents noise measurements from a flow sensor. The pump speed profiles used were ramp and stair step functions. These are speed profiles commonly used in in-vivo experiments. Physiological parameter changes encompass different contractility $\left(E_{\max }\right)$ and preload $\left(R_{S}\right)$ conditions. Simulations with noisy pump flow were conducted to investigate under what levels of SNR in the pump flow signal the suction detector classification is still accurate. Suction was evaluated based on $\Delta P=L V P-P I P$ as described in Section 5.1.

\subsubsection{Physiologic parameter change}

The physiologic parameters considered in these tests were contractility and preload. Contractility is related to the "pumping strength" of the natural heart. In our model, the following contractility states were evaluated by varying the value of $E_{\max }$ : 
- "normal" heart, $E_{\max }=2.0 \mathrm{mmHg} / \mathrm{ml}$;

- "sick" heart (reduced strenght), $E_{\max }=1.0 \mathrm{mmHg} / \mathrm{ml}$;

- "very sick" heart, $E_{\max }=0.6 \mathrm{mmHg} / \mathrm{ml}$;

Preload changes were used to assess the suction model response to changes in the patient's level of activity. In our cardiovascular-pump model, these changes can be simulated by increasing or decreasing the value of the resistor $R_{S}$. As $R_{S}$ decreases, the flow of blood returning to the left atrium (capacitance $C_{R}$ in our model) increases. Thus, left atrial pressure (LAP) increases, and so does the preload of the heart. The following activity states were considered (Table 15): "baseline" $\left(R_{S}=1.0 \mathrm{mmHg} . \mathrm{s} / \mathrm{ml}, \mathrm{HR}=75 \mathrm{bpm}\right)$, "hypertension" $\left(R_{S}=1.2 \mathrm{mmHg} . \mathrm{s} / \mathrm{ml}, \mathrm{HR}=75 \mathrm{bpm}\right)$, "light exercising" $\left(R_{S}=0.8 \mathrm{mmHg} . \mathrm{s} / \mathrm{ml}, \mathrm{HR}=\right.$ 90bpm), "strenuous exercising" $\left(R_{S}=0.6 \mathrm{mmHg} . \mathrm{s} / \mathrm{ml}, \mathrm{HR}=135 \mathrm{bpm}\right)$. The heart rate values were adopted from [60] and the $0.6 \mathrm{mmHg} . \mathrm{s} / \mathrm{ml}$ value for resistor $R_{S}$ is probably not physiologically likely to occur. Moreover, in the real world, a very sick patient would never be submitted to "strenuous exercising". Being able to test this condition through simulations, without jeopardizing the patient's health, illustrates the benefits of the simulation approach.

In addition, the healthy heart with ventricular assistance was included as suggested in [60]. This case actually happens during in-vivo animal studies prior to drug administration (e.g. esmolol) that weakens the ventricle, and in patients when the left ventricle recovers.

The twelve conditions described in Table 15 were simulated using a ramp speed profile. In the first 10 seconds of simulation, pump speed was constant at $\omega_{0}=8.7 \mathrm{krpm}$. Then, pump speed was increased until $14.0 \mathrm{krpm}$ and subsequently decreased to the initial value, $\omega_{0}$. This was done to assure that all case scenarios were tested for both increasing and decreasing pump speeds to match experimental protocols. Figures 53 and 54 show the results for the healthy heart. The first panel is pump speed, which was changed as previously described. The second panel is pump flow. The last two panels show the discriminant scores and the classification result from both the DA model and the expert.

Because preload decreases and heart rate increases with the level of exercise, the peakto-peak amplitude of pump flow decreases. For the cases of low heart rate (baseline and hypertension), the aortic valve is still opening and closing for a certain period of time for the healthy heart. However, as the heart rate increases, the aortic valve does not open, even 
Table 15: Parameter changes to evaluate the suction detector

\begin{tabular}{|c|ll|}
\hline $\begin{array}{c}\text { CONTRACTILITY STATE } \\
\text { (relates to the native } \\
\text { heart pumping strength) }\end{array}$ & \multicolumn{2}{|c|}{$\begin{array}{c}\text { PRELOAD CONDITIONS } \\
\text { (relate to the patient's } \\
\text { level of activity) }\end{array}$} \\
\hline \hline \multirow{2}{*}{ "normal heart" } & hypertension & $R_{S}=1.2, \mathrm{HR}=75$ \\
$E_{\text {max }}=2.0$ & baseline & $R_{S}=1.0, \mathrm{HR}=75$ \\
& light exercise & $R_{S}=0.8, \mathrm{HR}=90$ \\
strenuous exercise & $R_{S}=0.6, \mathrm{HR}=135$ \\
\hline "sick heart" & hypertension & $R_{S}=1.2, \mathrm{HR}=75$ \\
$E_{\text {max }}=1.0$ & baseline & $R_{S}=1.0, \mathrm{HR}=75$ \\
& light exercise & $R_{S}=0.8, \mathrm{HR}=90$ \\
& strenuous exercise & $R_{S}=0.6, \mathrm{HR}=135$ \\
\hline "very sick heart" & hypertension & $R_{S}=1.2, \mathrm{HR}=75$ \\
$E_{\text {max }}=0.6$ & baseline & $R_{S}=1.0, \mathrm{HR}=75$ \\
& light exercise & $R_{S}=0.8, \mathrm{HR}=90$ \\
& strenuous exercise & $R_{S}=0.6, \mathrm{HR}=135$ \\
\hline
\end{tabular}

at low speeds, because the systolic period decreases when heart rate increases.

Figures 55 through 58 show the simulation results for the sick and very sick hearts, respectively. Major differences are only in the pump flow signal. Due to the reduced contractility, peak-to-peak amplitude of pump flow decreases as $E_{\max }$ decreases. With regards to the discriminant scores, they behave in a similar fashion for all three contractility conditions, i.e., $D S_{1}$ is negative and $D S_{2}$ is positive when suction is absent, both scores are positive when severe suction occurs, and a change in signal takes place when moderate suction is happening, i.e., $D S_{1}$ is positive and $D S_{2}$ is negative. These are shown in Figures 53-58, third panel. 

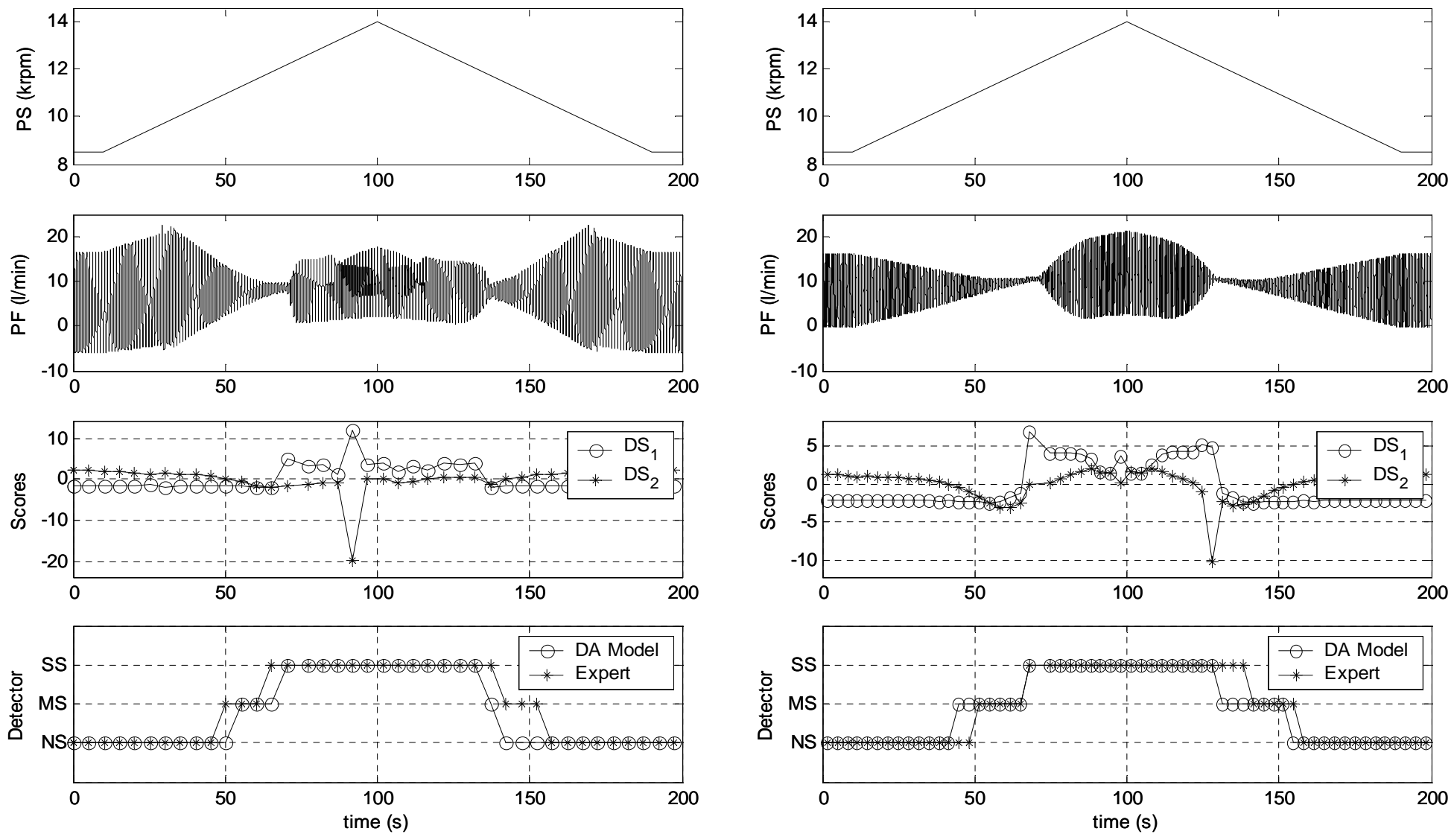

(a) baseline, $R_{S}=1.0 \mathrm{mmHg} . \mathrm{s} / \mathrm{ml}, \mathrm{HR}=75 \mathrm{bpm}$

(b) light exercise, $R_{S}=0.8 \mathrm{mmHg} . \mathrm{s} / \mathrm{ml}, \mathrm{HR}=90 \mathrm{bpm}$

Figure 53: Test result of parameter changes for the healthy heart $\left(E_{\max }=2.0 \mathrm{mmHg} / \mathrm{ml}\right)$. Panels from the top are PS (Pump Speed), PF (Pump Flow), Discriminant Scores and DA model and Expert classification. 

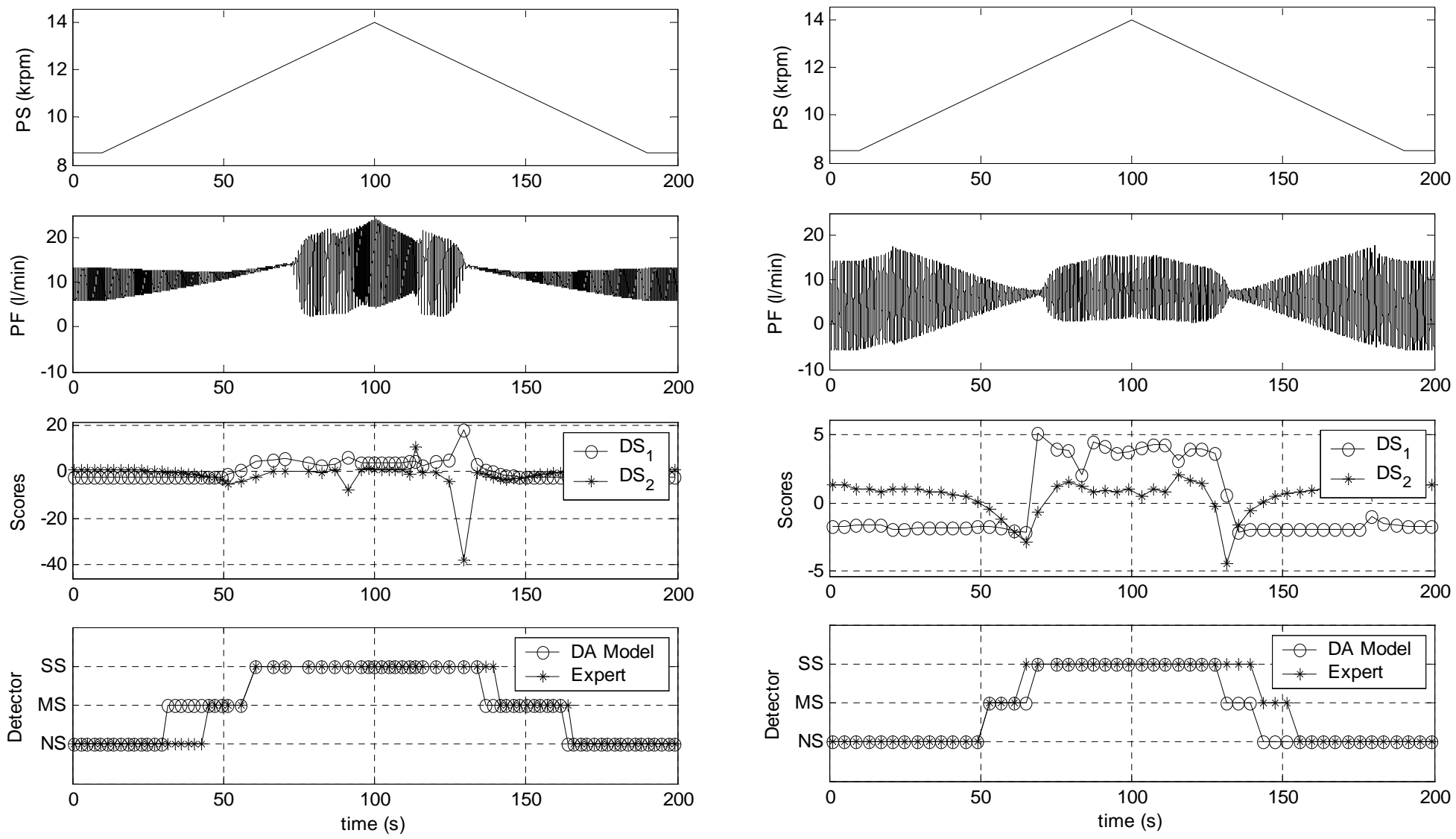

(a) heavy exercise, $R_{S}=0.6 \mathrm{mmHg} . \mathrm{s} / \mathrm{ml}, \mathrm{HR}=135 \mathrm{bpm}$

(b) hypertension, $R_{S}=1.2 \mathrm{mmHg} . \mathrm{s} / \mathrm{ml}, \mathrm{HR}=75 \mathrm{bpm}$

Figure 54: Test result of parameter changes for the healthy heart $\left(E_{\max }=2.0 \mathrm{mmHg} / \mathrm{ml}\right)$. Panels from the top are PS (Pump Speed), PF (Pump Flow), Discriminant Scores and DA model and Expert classification. 

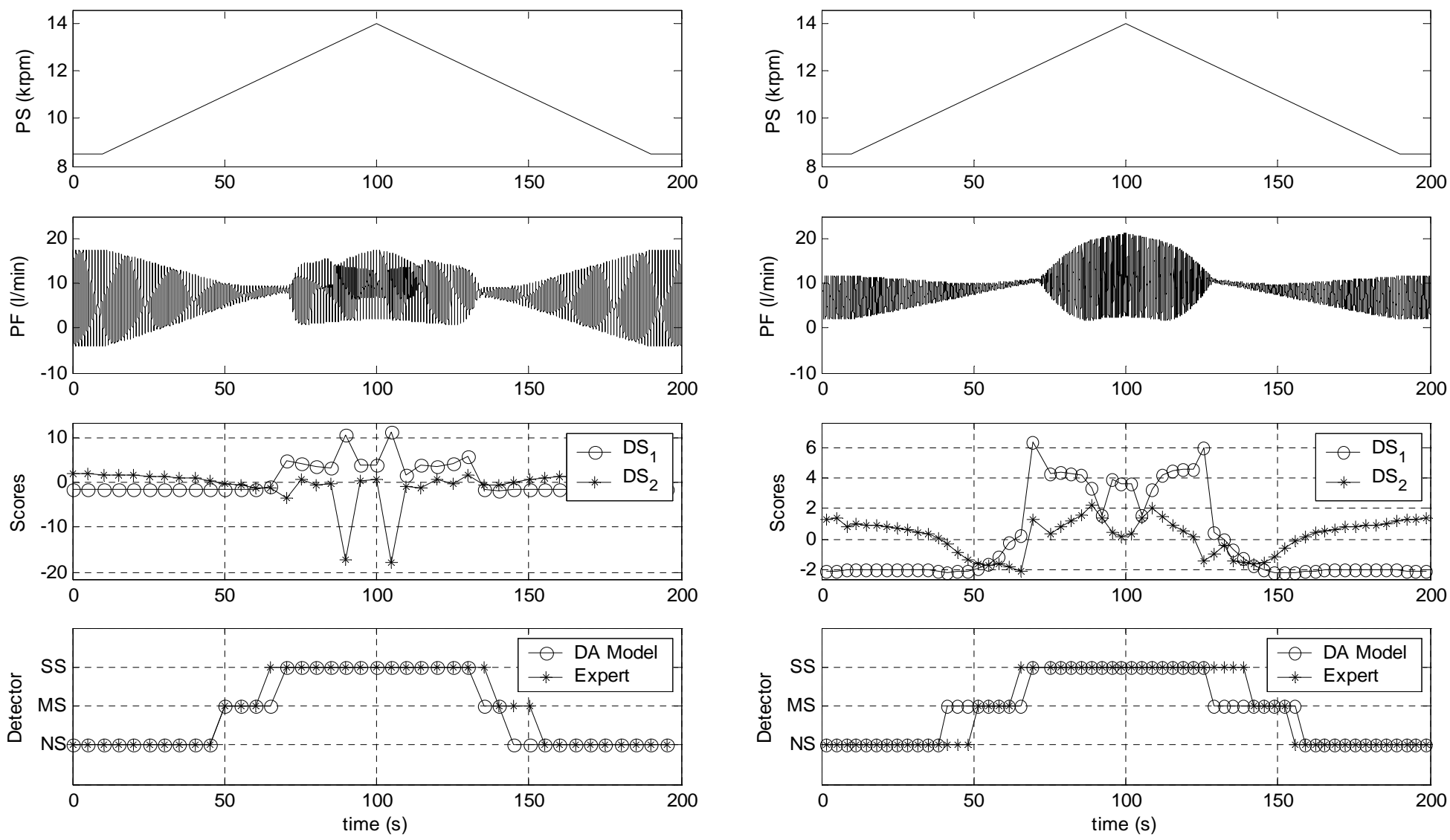

(a) baseline, $R_{S}=1.0 \mathrm{mmHg} . \mathrm{s} / \mathrm{ml}, \mathrm{HR}=75 \mathrm{bpm}$

(b) light exercise, $R_{S}=0.8 \mathrm{mmHg} . \mathrm{s} / \mathrm{ml}, \mathrm{HR}=90 \mathrm{bpm}$

Figure 55: Test result of parameter changes for the sick heart $\left(E_{\max }=1.0 \mathrm{mmHg} / \mathrm{ml}\right)$. Panels from the top are PS (Pump Speed), PF (Pump Flow), Discriminant Scores and DA model and Expert classification. 

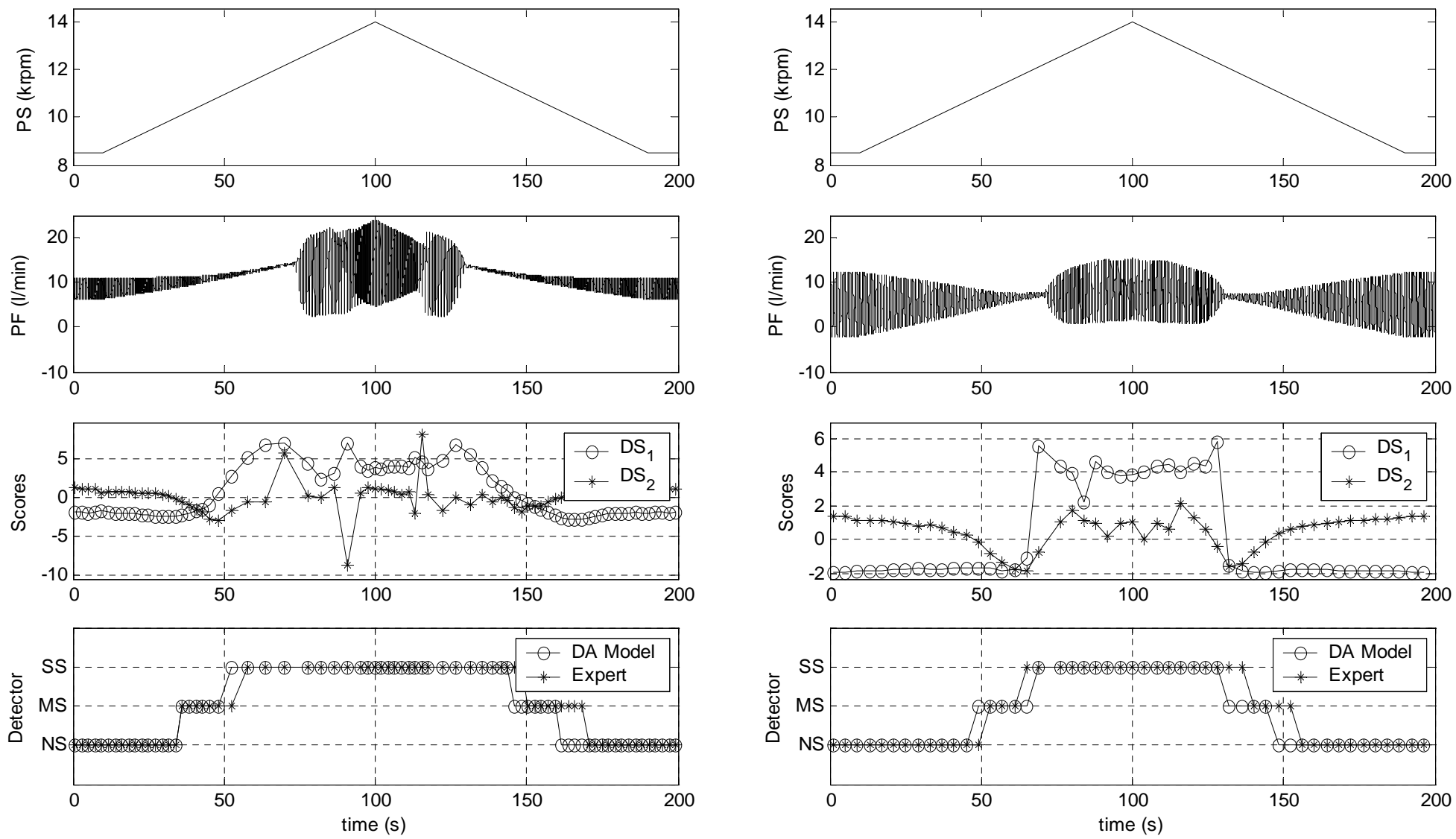

(a) heavy exercise, $R_{S}=0.6 \mathrm{mmHg} . \mathrm{s} / \mathrm{ml}, \mathrm{HR}=135 \mathrm{bpm}$

(b) hypertension, $R_{S}=1.2 \mathrm{mmHg} . \mathrm{s} / \mathrm{ml}, \mathrm{HR}=75 \mathrm{bpm}$

Figure 56: Test result of parameter changes for the sick heart $\left(E_{\max }=1.0 \mathrm{mmHg} / \mathrm{ml}\right)$. Panels from the top are PS (Pump Speed), PF (Pump Flow), Discriminant Scores and DA model and Expert classification. 

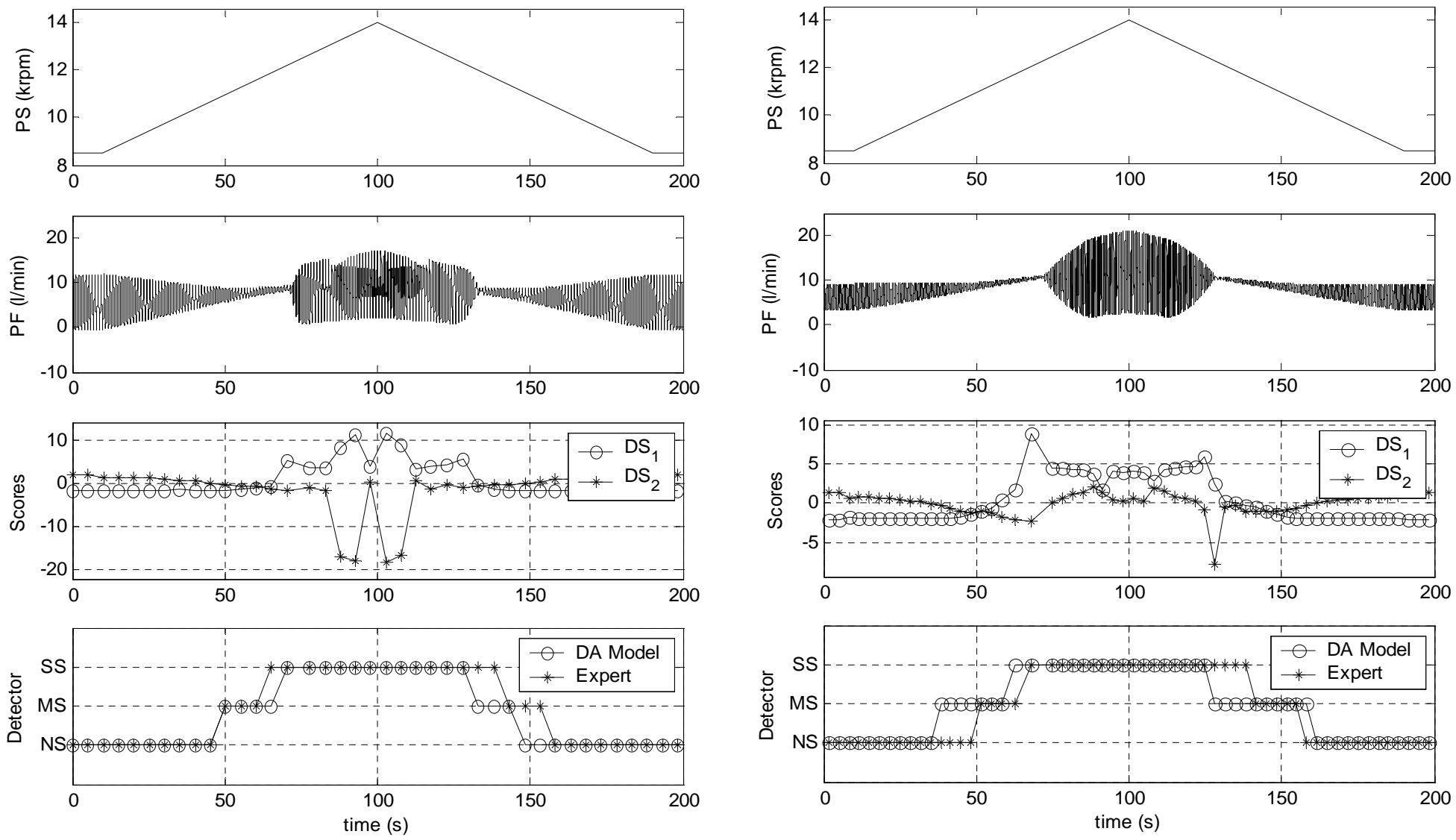

(a) baseline, $R_{S}=1.0 \mathrm{mmHg} . \mathrm{s} / \mathrm{ml}, \mathrm{HR}=75 \mathrm{bpm}$

(b) light exercise, $R_{S}=0.8 \mathrm{mmHg} . \mathrm{s} / \mathrm{ml}, \mathrm{HR}=90 \mathrm{bpm}$

Figure 57: Test result of parameter changes for the very sick heart $\left(E_{\max }=0.6 \mathrm{mmHg} / \mathrm{ml}\right)$. Panels from the top are PS (Pump Speed), PF (Pump Flow), Discriminant Scores and DA model and Expert classification. 

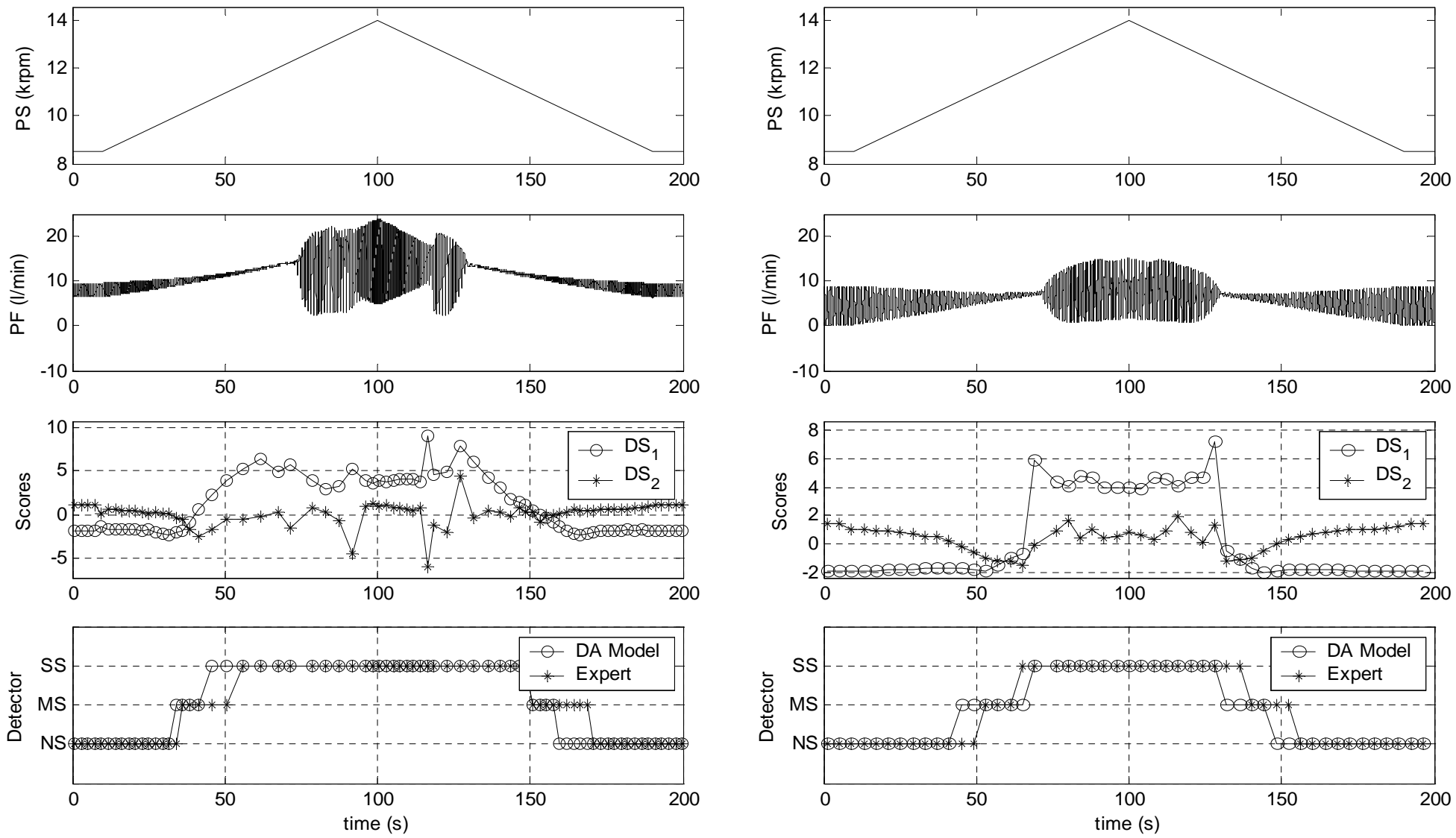

(a) heavy exercise, $R_{S}=0.6 \mathrm{mmHg} . \mathrm{s} / \mathrm{ml}, \mathrm{HR}=135 \mathrm{bpm}$

(b) hypertension, $R_{S}=1.2 \mathrm{mmHg} . \mathrm{s} / \mathrm{ml}, \mathrm{HR}=75 \mathrm{bpm}$

Figure 58: Test result of parameter changes for the very sick heart $\left(E_{\max }=0.6 \mathrm{mmHg} / \mathrm{ml}\right)$. Panels from the top are PS (Pump Speed), PF (Pump Flow), Discriminant Scores and DA model and Expert classification. 
To evaluate how these changes impact the suction detector performance, the hit-rate of the detector was calculated for each test condition. Figure 59 shows that there are not major differences within and across the contractility states considered. Indeed, for all contractility conditions, the hit-rate of the detector lies in the interval $[82 \%, 90 \%]$. The confusion matrices of each test condition are shown in Appendix C.

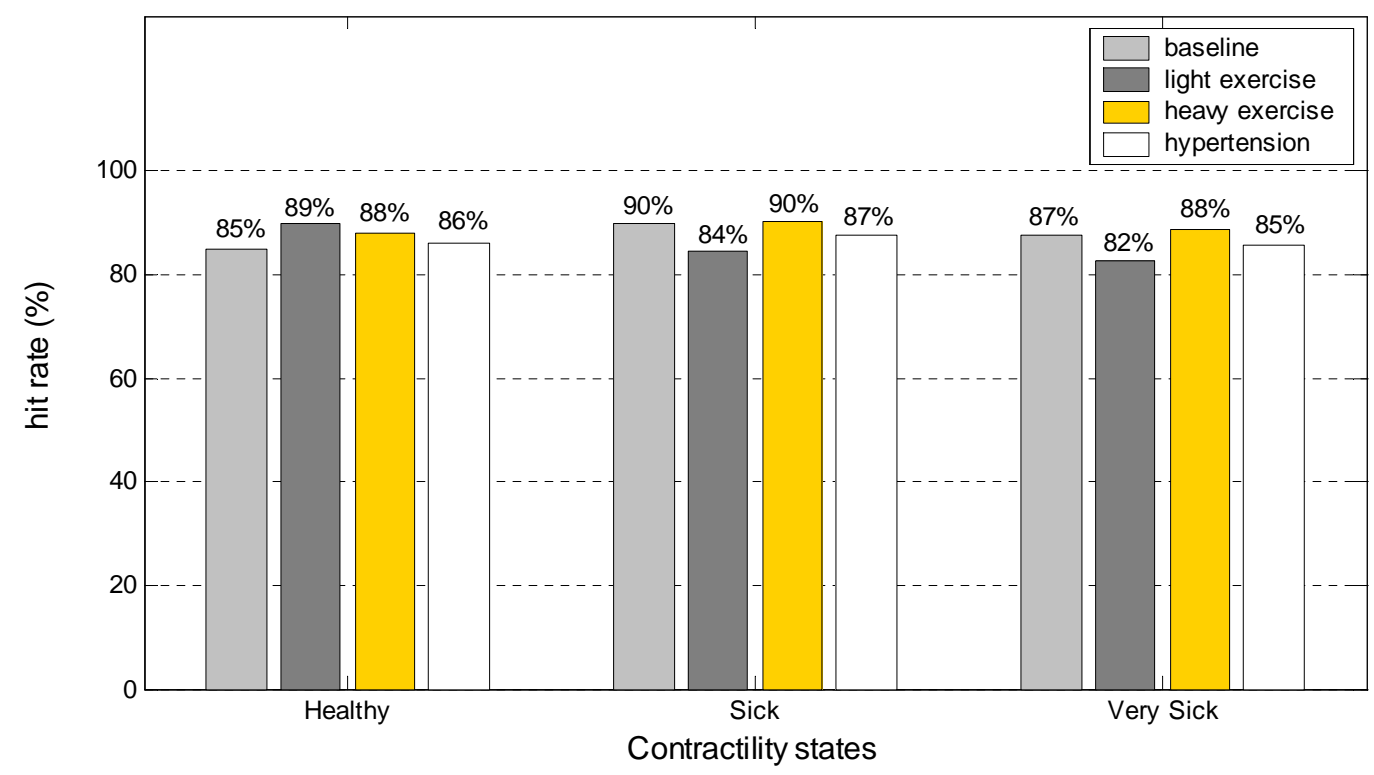

Figure 59: Detector hit-rate as a function of contractility state

\subsubsection{Robustness to Noise}

In order to assess the detector performance when a noisy pump flow signal is presented to its input, a simulation study with white gaussian noise (signal $v(n)$ in Figure 52) added to the pump flow signal was conducted. The strategy used to evaluate how noise impacts the hit-rate of the detector was to increase the SNR level and measure the hit-rate to determine under which noise conditions the detector's hit-rate is not better than the proportional chance criteria. As in the previous test, three contractility states were considered, i.e., healthy heart, sick heart and very sick heart.

To provide a sense of how comparable with in-vivo experimental data these simulated noisy pump flows are, Figure 60 shows three panels of pump flow. Panel (a) presents in- 
vivo data, and panels (b) and (c) show pump flow signals from simulations with $20 \mathrm{~dB}$ and $10 \mathrm{~dB}$ levels of SNR respectively. Comparing them, we can noticed that the in-vivo data is reasonably "clean", and that much less favorable conditions were considered in simulation.

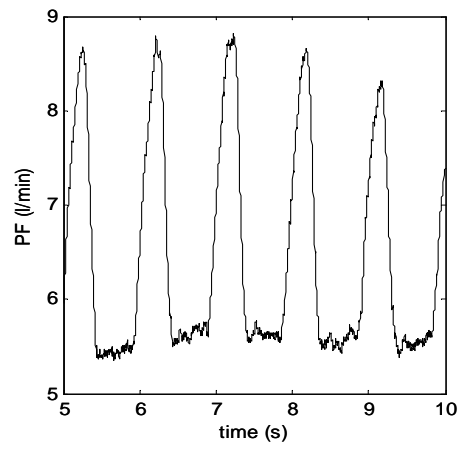

(a) raw in-vivo PF

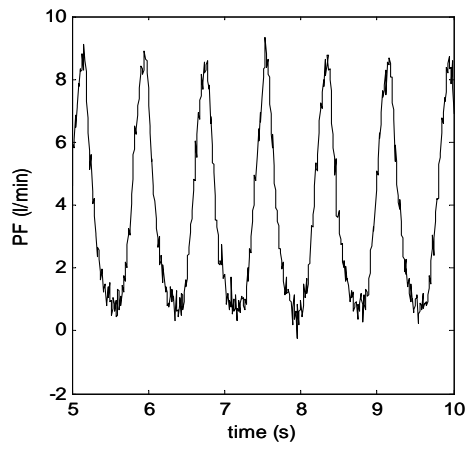

(b) simulated PF, SNR $=20 \mathrm{~dB}$

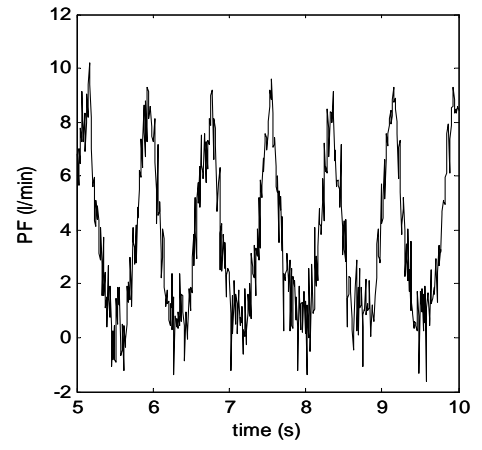

(c) simulated PF, SNR $=10 \mathrm{~dB}$

Figure 60: Comparison between raw and noisy simulated pump flow

Figure 61 summarizes the simulation results. The horizontal line at the $37 \%$ accuracy level corresponds to the proportional chance criteria in this case. Noise was added to the pump flow signal, according to the following SNR values: 100, 80, 60, 40, 20, 10, 5 and $2 \mathrm{~dB}$. The 100dB level, which corresponds to no noise added, was included for comparison purposes. There are no pronounced differences in accuracy from SNR levels 80 to 20dB. At $\mathrm{SNR}=20 \mathrm{~dB}$, the detector performances of the sick (solid line with square marks, $E_{\max }=$ $1.0 \mathrm{mmHg} / \mathrm{ml}$ ) and very sick (solid line with triangle marks, $E_{\max }=0.6 \mathrm{mmHg}$ ) hearts were about the same, presenting a hit-rate of $87 \%$, as compared with the healthy heart (solid line with circle marks, $E_{\max }=2.0 \mathrm{mmHg} / \mathrm{ml}$ ), whose hit-rate was $85 \%$. However, as more noise is added to the pump flow signal, the hit-rates of the healthy and sick heart decrease in a similar fashion and for the very sick heart the detector's performance deteriorates more rapidly when $\mathrm{SNR} \leq 10 \mathrm{~dB}$. At the $2 \mathrm{~dB}$ level, the detector can do no better than the chance criteria for the sick and very sick hearts. 


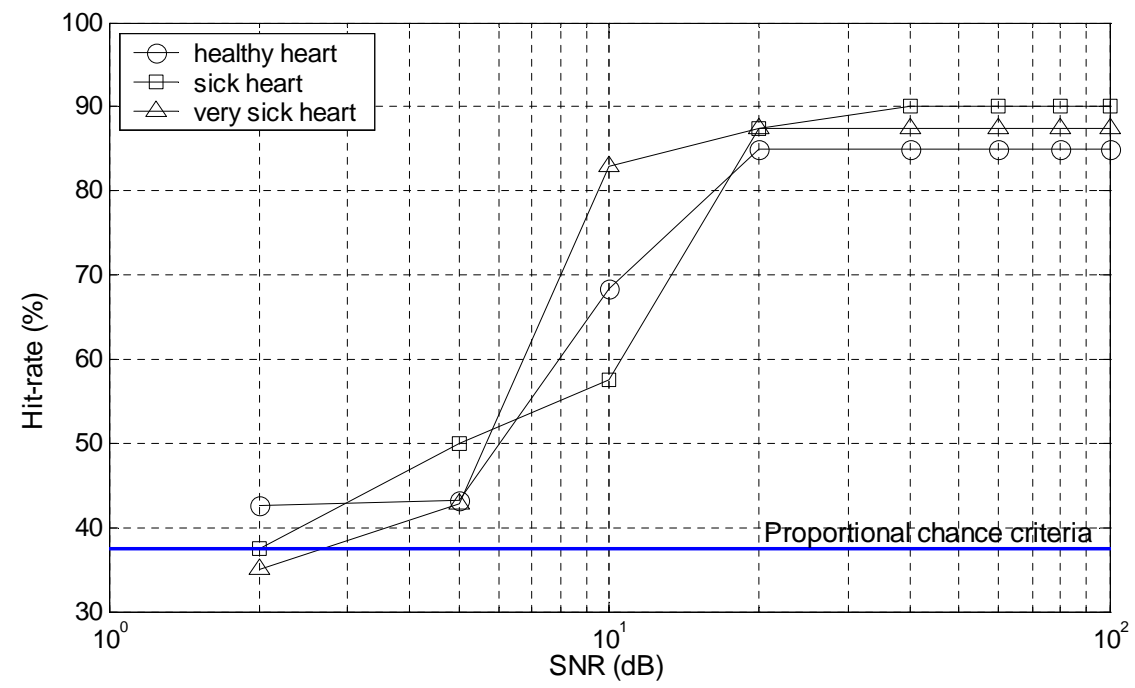

Figure 61: Detector hit-rate as a function of SNR

\subsection{HOW DO THE DISCRIMINANT SCORES BEHAVE OVER TIME?}

In this section we examine how the discriminant scores behave in time. Our goal is to determine whether the discriminant scores can be used as control signals to drive a physiological controller for the LVAD. In other words, can we use the discriminant scores to adjust the set point of pump speed, so that suction is avoided? To accomplish this, the discriminant scores should be "smooth" functions of time and "monotonic" functions of speed. By smooth, we mean that these signals should be "relatively continuous", without presenting abrupt changes in amplitude over time. Monotonicity here means that the operating point of the pump in the discriminant scores plane should only move from one region to another if pump speed has been increased or decreased. Otherwise, the operating point should stay in one of the three regions (NS, MS, or SS). That is, we do not want the point to "jump" randomly in the discriminant scores plane, but to move systematically through it, as pump speed changes.

Recall that pump flow samples were randomly drawn from a data base to design the classifier. Because of this, time information was not considered in the design process. Indeed, 
every sample in the data base is a 5 heart-beats long window of pump flow, and those windows come from 35 data files recorded during animal experiments. The DA model does not know from which particular file a sample comes or the location in time of a particular window within its original file. We can investigate how the discriminant scores behave over time by applying the designed DA model to the data files, instead of to the samples in the test set. Consider the results of applying the DA model to the data File \# 20. It is a 350-second segment of recorded hemoynamic variables, with several episodes of severe suction caused by overpumping (high pump speed).

As shown in Figure 62 (a), pump speed is increased from 1, 900 to $2950 \mathrm{rpm}$, in the first $85 \mathrm{~s}$ of simulation. Pump speed started low, with no suction, but at $60 \mathrm{~s}$, the pump status went from normal (NS) to moderate suction (MS) according to the expert (see Figure 62 (c), dark line). The vertical lines labeled by capital letters at the top of the first panel indicate transitions from one pump state to another, according to the expert classification. These letters will be used to identify pump state transitions on Figure 63. There is one transition from NS to MS indicated by A, at $\omega=2,500 \mathrm{rpm}$. Transitions from MS to SS occurred at B, D, F, H and J. In all these transitions, we had $\omega \geq 2,900 \mathrm{rpm}$. Transitions from SS to MS occurred at C, E, G, and I when pump speed was decreased to $\omega \leq 2,800 \mathrm{rpm}$. Notice that the suction detector identified correctly all severe suction episodes except the one at $\mathrm{H}$ $(t=240 \mathrm{~s})$. (Compare the expert and DA model output in Figure $62(\mathrm{c}))$.

Figure 62 (d) shows how the discriminant scores vary with time for this paricular test. The first discriminant score $\left(D S_{1}\right)$ is negative when either NS or MS patterns are occurring. If $D S_{1}$ and $D S_{2}$ are positive, severe suction is occurring. Figure 63 reveals better what happens with pump status in the $D S_{1} \times D S_{2}$ plane, as time goes by. The discriminant scores plane can be divided into three regions according to the aforementioned groups. Since the group means in that space are "well" separated (indicated by the dark crosses), lines can be determined to separate the three groups from one another. Note that unless pump speed changes, the operating point tends to stay within a local region (this is indicated by the dashed lines connecting consecutive time windows). Indeed, to consider the discriminant scores as potential candidates for a control application, we do not want for them to change drastically, making the operating point to "jump" from one region to another frequently. 
The behavior of the discriminant scores shown for the data File \# 20 is typical, since the discriminant scores from others data records behave in a similar fashion.
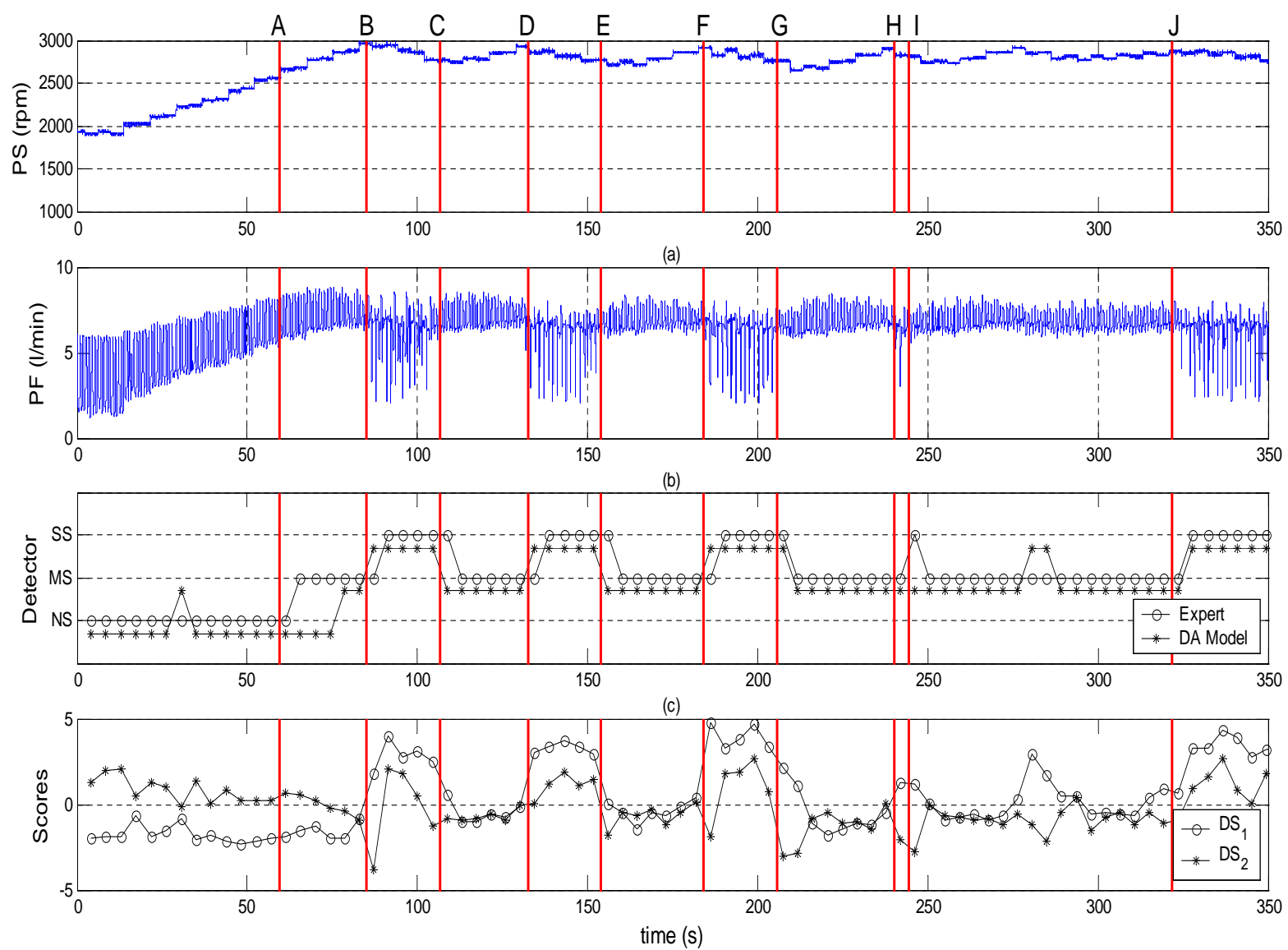

Figure 62: DA results for data File \# 20. (a) Pump Speed, (b) Pump Flow, (c) Detector output and Expert decision, and (d) Discriminant Scores. Capital letters indicate pump state transitions: A, NS $\rightarrow$ MS; B, D, F, H and J: MS $\rightarrow$ SS; C, E, G, I: SS $\rightarrow$ MS 


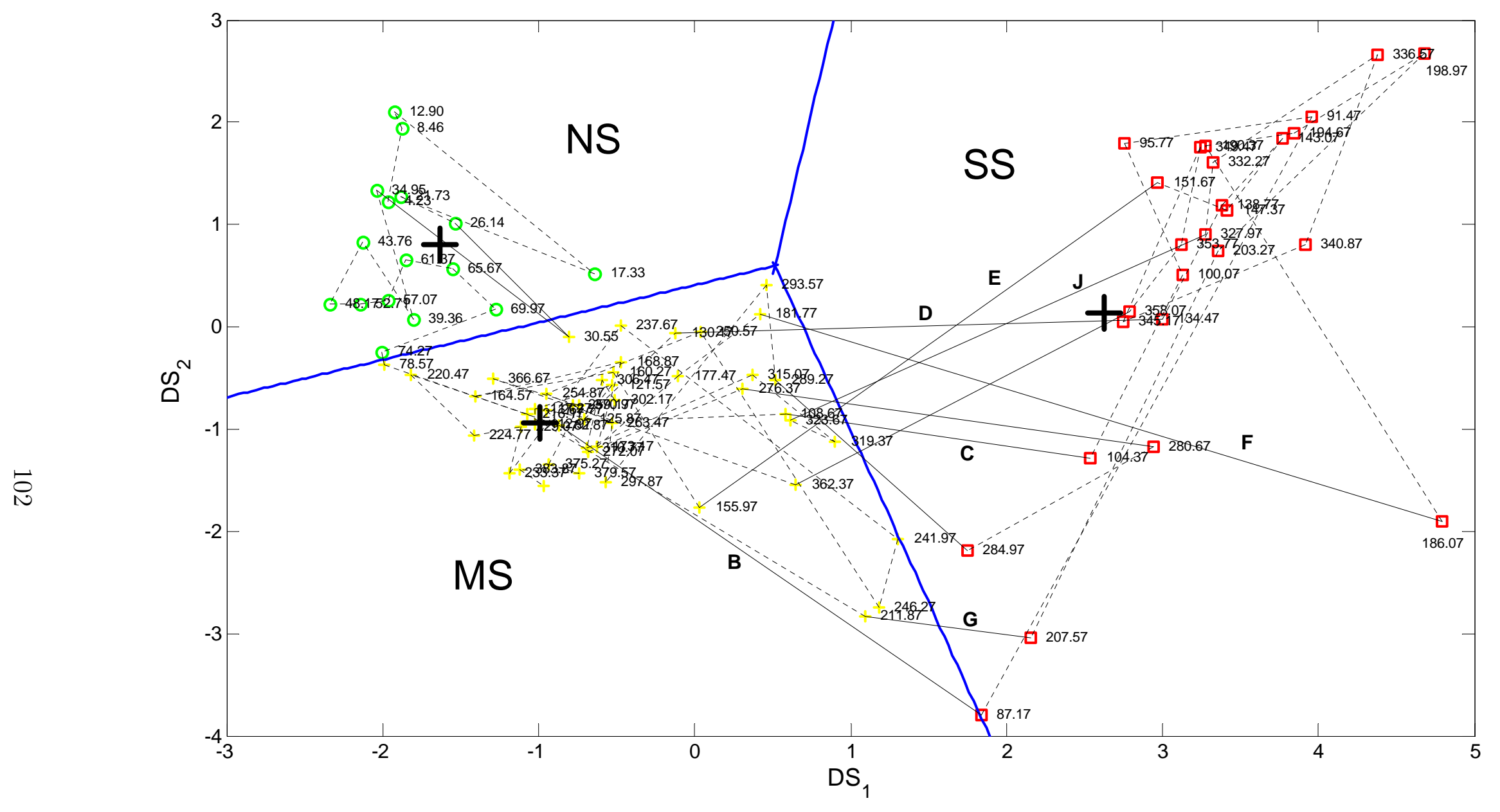

Figure 63: Pump status point in the discriminant score plan. The dark crosses represent the group means. The small numbers at the side of the points represent the time information. Dashed lines connect consecutive time windows. Solid lines represent transitions in pump state, and capital letters indicate crossing boundaries according to changes shown in Figure 62. 


\subsection{A RULE-BASED CONTROLLER FOR ROTARY BLOOD PUMPS}

The control problem for rotary ventricular assist devices is to set pump speed such that cardiac output (pump flow) and pressure perfusion are within acceptable physiological ranges. Since blood flow demand varies according to the patient's level of activity, an adaptive controller is desirable to adjust pump speed.

When operated in a open-loop fashion, the desired operating point of the rotary VAD may be identified by a clinician or technical personnel. This control strategy severely compromises the patient's quality of life because adjustments in pump speed cannot be made when technical personnel are absent, regardless of physiologic changes of the patient.

Due to the lack of reliable pressure and flow sensors for long term implants, observability of state variables is an issue for controlling rotary VADs. This problem compelled researchers to investigate alternative approaches to estimate these variables. For instance, estimation of pump flow and pressure difference variables from pump motor current and speed was suggested by Schima et al [46]. Yu et al [37] proposed a Kalman filter to estimate systemic vascular bed parameters for real time control applications of artificial hearts.

A heart rate based controller for rotary VADs was introduced by Golding at al. [58]. They demonstrated that flow and demand are linearly dependent on heart rate. Thus, using heart-rate as a control signal, they determined the appropriate pump speed that provides the required blood flow. Even though this approach seems appealing, it needs a precise mapping between heart-rate and flow, which may be difficult to obtain in clinical applications. If the

patient's heart rate is not stable (e.g., arrhythmia), harmful control signals may be generated. Moreover, this approach does not guarantee the absence of suction events.

Giridharan et al $[59,60]$ developed a pressure based controller. Their approach was to adapt pump flow according to the physiological status of the patient (i.e, rest, exercising, 
walking) by maintaining a constant average pressure difference $(\Delta P)$ between the left ventri-

cle and the aorta. However, keeping $\Delta P$ constant does not assure adequate cardiac output and suction detectors are needed for this approach as well.

Controllers that set pump speed to avoid suction are generally based on some index or feature extracted from a signal (e.g., pump speed, pump current or pump flow) which indicate whether or not suction is present. Choi et al [7] proposed a proportional-integral type fuzzy logic controller to adjust pump speed based on a pulsatility index, derived from the pump flow signal. However, suction may occur if the reference pulsatility value is not properly set.

A possible control approach to avoid entering the suction region is to embed suction detectors in a control supervisor structure, as suggested in [4]. That approach takes into account all criteria of interest to clinicians, i.e, adapting cardiac output according to the patient's level of activity, sustainable pressure perfusion, and preload pressure below a maximum of $15 \mathrm{mmHg}$ to avoid pulmonary edema. However, such an approach requires extensive information regarding the pump status as well as real time estimation of the hemodynamic parameters of the patient.

In this chapter, a new control system for rotary VADs is presented. The approach taken here is to use the discriminant scores signals from the suction detector as inputs to a rulebased controller. The controller uses fuzzy logic to automatically adjust pump speed so as to avoid suction, while keeping cardiac output and perfusion pressure within physiologic ranges. This chapter is organized as follows. Section 6.1 justifies the application of fuzzy logic to this particular control problem. Section 6.2 presents the controller design details, and Section 6.3 presents the simulation results to characterize the performance of the controller.

\subsection{WHY A RULE-BASED CONTROLLER ?}

Besides the observability problem previously mentioned, two others reasons motivated us to pursue a rule-based controller. First, the control problem of rotary VADs is ill posed [39]. Indeed, generally speaking, control systems are designed to regulate the control variable to a 
given reference value or "set point". In our case, the body's demand for blood would be that reference. However, such demand is not easily determined. It depends on preload, afterload, contractility state of the native heart and level of activity of the patient. Even psychological factors could influence blood flow requirements. Hence, it would be difficult to implement a control strategy for rotary VADs based on traditional control approaches, such as dynamic programming, optimal control, or the maximum principle. Fuzzy logic has been successfully applied to complex systems that show this level of parameter uncertainty [26, 27].

Secondly, the expert's classification of suction status is linguistic in nature. Indeed, when talking about pump flow patterns, experts are certain about no suction and severe suction (which are two extreme conditions) to a high degree. However, intermediate patterns are usually described as "most probably suction", or even "not decidable" [11]. For these patterns, the expert's certainty about classification is not as high as it is for severe suction patterns or no suction. Since the discriminant scores from the suction detector are "well behaved" signals in time and behave in a "monotonic" fashion with respect to speed, it seems reasonable to use them to identify an operating point for pump speed. Fuzzy logic provides a method to combine the two scores and to translate them to the linguistic concept of suction and non-suction provided by the expert.

The idea of having a classifier "driving" a controller was proposed by $\mathrm{Fu}[61,62]$. He advocated the use of a supervisor to estimate the states (or unknown information) of a plant and feed that estimated information to a controller. He pointed out that similar control situations may be grouped to form a class of control situations. Since these different classes of control situations have to be identified, a pattern classifier is required when designing this type of learning control system. The suction detector plays this role in our approach, since it can identify the pump status based on a classification of the pump flow patterns. Moreover, a reference signal is not provided for the reasons previously discussed. The detector provides such a reference, based on the discriminant scores information. 


\subsection{RULE-BASED CONTROLLER DESIGN}

A rule-based controller for rotary VADs was designed, following Mamdani's approach [29]. As stated in Section 3.3.1, the design task of such a controller encompasses three main tasks: the design of a rule-base, the design of the membership sets of the input and output variables and the choice of a defuzzification method. In this application, the discriminant scores from the DA model are the input variables. These scores indicate the pump status as either NS, MS or SS. The output variable, $\delta_{\omega}$, is a percent change in pump speed on the interval $\left[\tau_{1}, \tau_{2}\right]$, where $\tau_{1}$ and $\tau_{2}$ are design parameters that determine the range of the output variable. Pump speed is updated according to

$$
w_{k+1}=w_{k}\left(1+\delta_{\omega}\right)
$$

where $\delta_{\omega}$ is the output of the rule-based controller in the $k^{\text {th }}$ time window. In our application, a time window is 5 heart-beats long. The initial pump speed, $\omega_{0}$, is a value given by the clinician. This value is usually estimated by a cardiac exam, which estimates the flow of blood returning to the patient's heart (venous return).

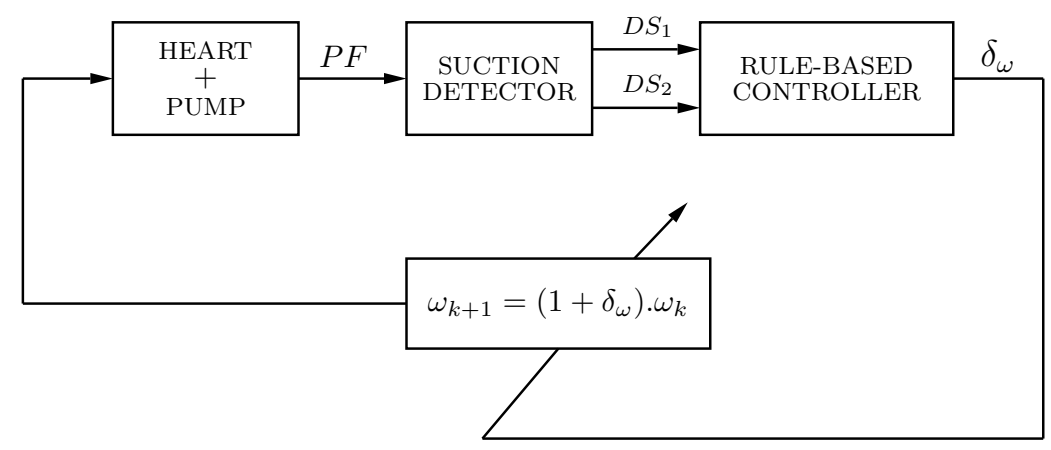

Figure 64: Pump speed update 


\subsubsection{Rule base design}

The number of input fuzzy sets defined on the input variables domain dictates the number of rules in the rule-base. To simplify the design task, assume that the number of fuzzy sets defined on both input variables is equal to $N$. This means that $N^{2}$ rules are needed in the rule-base. An immediate consequence of having many sets defined in the input variables domain is that more rules are needed for the rule base, making the expert's design task more demanding.

The rules are usually organized in a bank of rules, the FAM bank. This bank associates input variables, $D S_{1}$ and $D S_{2}$ with the output variable, $\delta_{\omega}$. The rule base is a mapping between input and output of the controller. It can also be seen as a nonlinear function $u=\Phi\left(D S_{1}, D S_{2}\right)$ that defines its transfer characteristic. A plot of $\Phi$ is usually referred as a "control surface" [26]. It is a mesh plot of the output $\delta_{\omega}$ as a function of the input variables, the discriminant scores, $D S_{1}$ and $D S_{2}$. The output values were calculated based on the defuzzification method presented in Section 3.3.1 and given as

$$
\delta_{\omega}=\frac{\sum_{k=1}^{m} c_{k} \cdot f_{k}}{\sum_{k=1}^{m} f_{k}}
$$

where $m$ is the number of fired rules, $c_{k}$ are the center of the membership functions corresponding to these rules and $f_{k}$ are their heights. The decision about which rule based should be adopted was made by examining the corresponding simulation results.

The number of input and output membership sets commonly used when designing fuzzy controller varies from 3 to 9 in most applications encountered in the literature [26, 27]. Parsimony should be used when defining these numbers because they determine the number of rules needed in the rule base. The number of membership sets of a variable should also reflect its granularity. The granularity of fuzzy sets reflects how "specific" they are [27]. For example, very narrow intervals are definitely more specific than broader ones. In the limit, if a single fuzzy set covers the entire variable range, then its granularity level attains a minimum.

In this approach, to design the rule base, three combinations of input-output sets were examined, as shown in Figure 65: (a) five input sets and three output sets; (b) five sets 
for all variables (input and output) and (c) seven sets for all variables (input and output). Combinations in which three membership sets were used for the input variables were not considered because only three membership sets cannot encode the linguistic information in the discriminant scores range. Using more than seven membership sets represents a higher granularity level than is expected to be necessary for this particular application. In the following test, $\delta_{\omega}$ was defined on the interval $[-0.05,0.05]$, i.e., $\tau_{1}=-0.05$ and $\tau_{2}=0.05$.
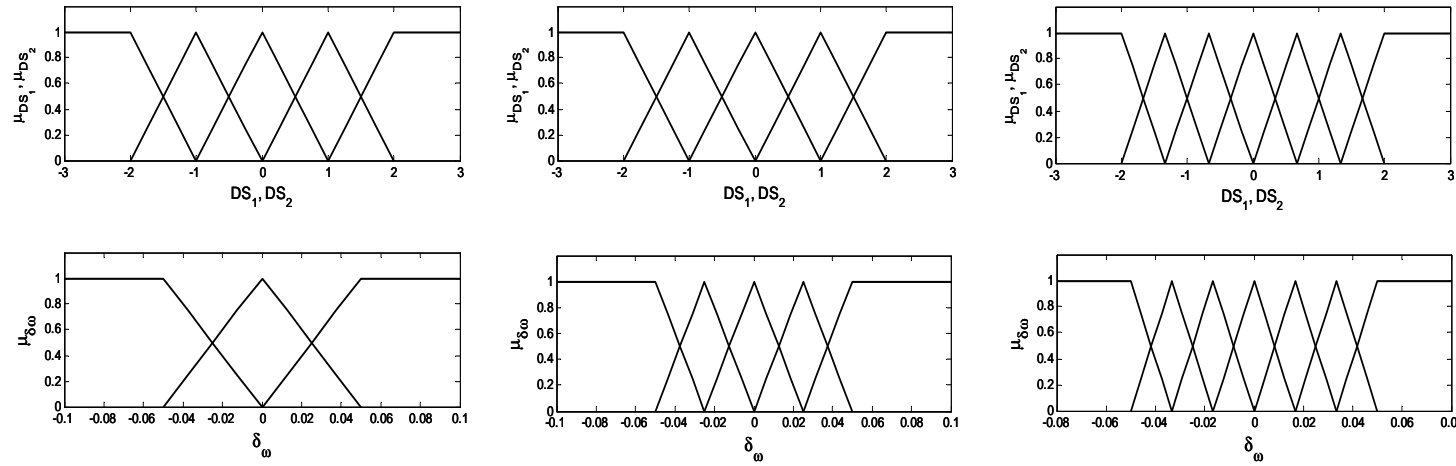

(a) three output sets

(b) five output sets

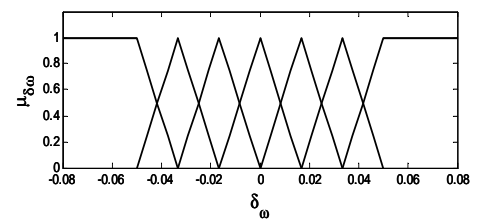

(c) seven output sets

Figure 65: Input-output sets examined to derive the rule base. Top panels are input sets definitions and bottom panels are output sets.

The control surfaces corresponding to each input-output scenario are shown in Figure 66 and their corresponding FAM banks in Appendix D. The criterion used to evaluate the control surfaces was based on the steady state response of the controller. Simulations were performed with both low $\left(\omega_{0}=8.5 \mathrm{krpm}\right)$ and high $\left(\omega_{0}=13.0 \mathrm{krpm}\right)$ initial pump speed. Figure 67 shows these results. Pump signals A, B and C were obtained using the control surfaces shown in Figure 66(a, b and c), respectively. When the initial speed is low, steady state at $\omega_{s s}=11 \mathrm{krpm}$ is reached in about $90 \mathrm{~s}$ for control surfaces (b) and (c). However, when using control surface (a), the pump speed signal oscillates around $\omega=11 \mathrm{krpm}$ value. Likewise, when $\omega_{0}$ was set high, control surface (a) makes the pump speed signal oscillate, whereas (b) and (c) reached steady state around $\mathrm{t}=160 \mathrm{~s}$.

Because pump speed was oscillatory when the rule base corresponding to control surface (a) was used, no further consideration was given to that rule base in the design process. 


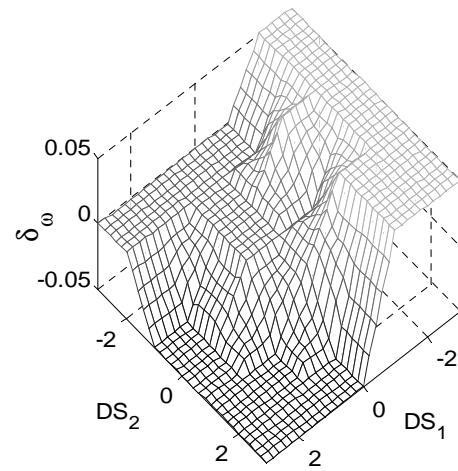

(a) three output sets

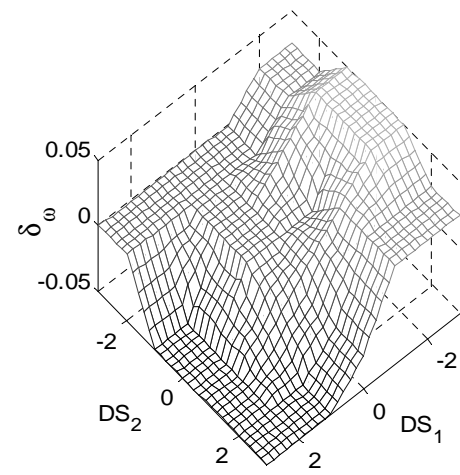

(b) five output sets

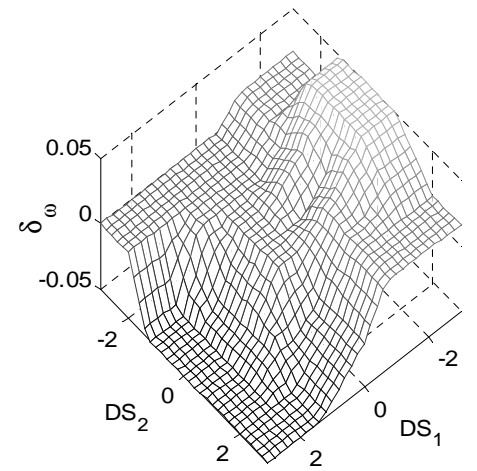

(c) seven output sets

Figure 66: Control surfaces

Control surface (c) updates pump speed in a very similar fashion of control surface (b). Moreover, the rule base corresponding to control surface (b) is more "appealing" from the rule base design standpoint, because it requires fewer rules, $5^{2}=25$, whereas control surface (c) needs a rule base with $7^{2}=49$ rules to be defined. As pointed out earlier, experts usually have difficulty in designing so many rules. Therefore, control surface (b) will be adopted in the final controller design.

The pump speed signal obtained using control surface (a) presented oscillatory behavior because the operating point of the system does not stay in a region where $\delta_{\omega}$ is zero. For instance, consider Figure 68 (a). The last five operating points (black crosses) in the simulation are shown on control surface (a) with the corresponding time information. Note that the point jumps from one site to another in the control surface, without settling down at $\delta_{\omega}=0$. In contrast, Figure 68(b) shows that when control surface (b) was used, the operating point moved smoothly, settling in a region where $\delta_{\omega}=0$. 

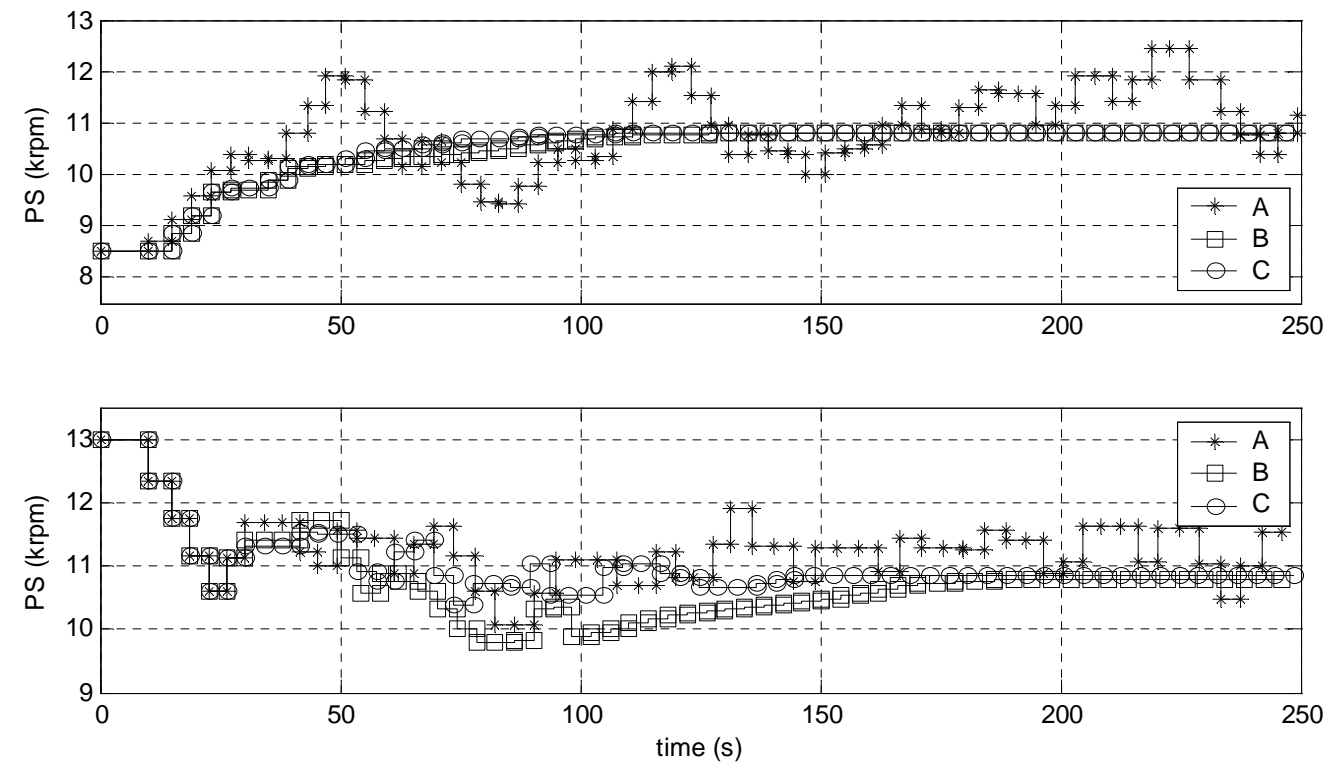

Figure 67: Simulation results of pump speed for control surfaces. Top panel shows low initial pump speed and bottom panels shows high pump speed.

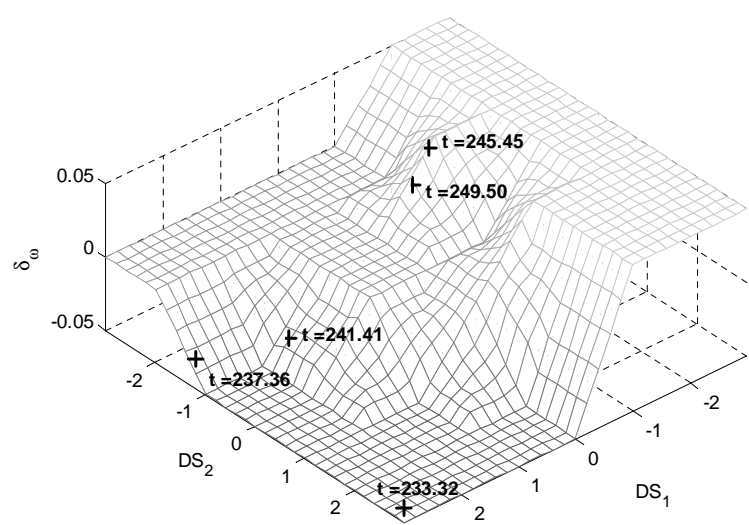

(a) using three output sets

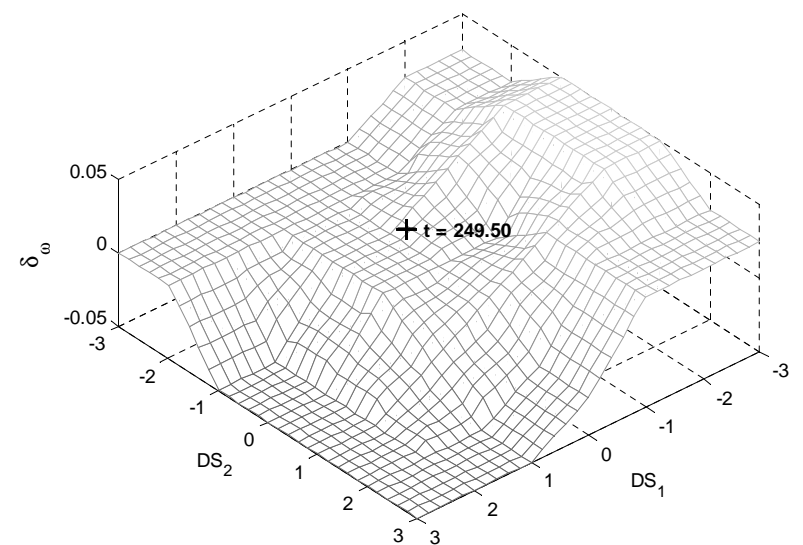

(b) using five output sets

Figure 68: Operating point on control surfaces. Only the last five points are shown with time information. The last five points are coincidents in (b). 


\subsubsection{Effects of Asymmetry in output sets}

Another important design issue is related to the transient response of the controller. Indeed, since severe suction is a deleterious condition for the patient, it is important to know how fast the controller can decrease pump speed, so as to drive the pump out of severe suction quickly. And, conversely, can the controller speed up the pump when a low flow condition is detected? One way to speed up the controller's response is by changing the design parameters $\left[\tau_{1}, \tau_{2}\right]$, which determine the range of the output variable, $\delta_{\omega}$. In this section, two different definitions for the output sets are considered: in the first case, we use $\tau_{1}=-0.05$ and $\tau_{2}=0.1$; in the second, $\tau_{1}=-0.1$ and $\tau_{2}=0.05$. These two case scenarios will be compared with the previous (symmetric) membership output set definitions shown in Figure 65(b), for which $\tau_{1}=-0.05$ and $\tau_{2}=0.05$. Both the support and the symmetry of the sets was changed to keep the crosspoint of adjacent membership sets at $\mu=0.5$ membership value, as shown in Figure 69. Controllers with the following goals will be compared:

\begin{tabular}{ccl} 
Controller & Output sets & Goal \\
\hline A & Figure 69(a) & Increases speed quickly when flow is low \\
B & Figure 69(b) & Decreases speed quickly when severe suction is detected \\
C & Figure 69(c) & Previous definition; "smooth" speed transitions \\
\hline
\end{tabular}
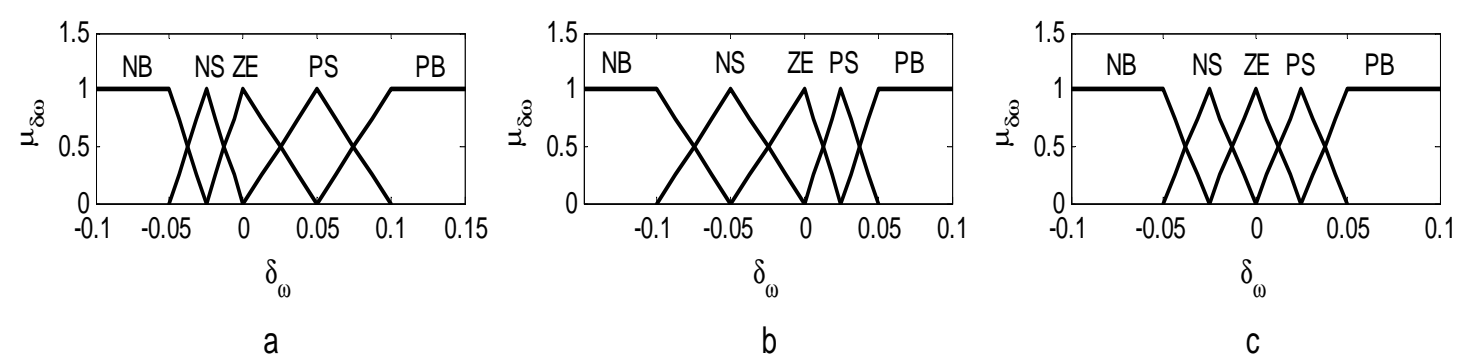

Figure 69: Output Membership sets

From the transfer characteristic standpoint, these new definitions of the $\delta_{\omega}$ membership sets change the shape of the control surface. Indeed, Figure 70 shows that for controller A, a $10 \%$ increase in pump speed can occur, depending on the antecedents of the fired rules. Controller B can produce a $-10 \%$ decrease in pump speed. 


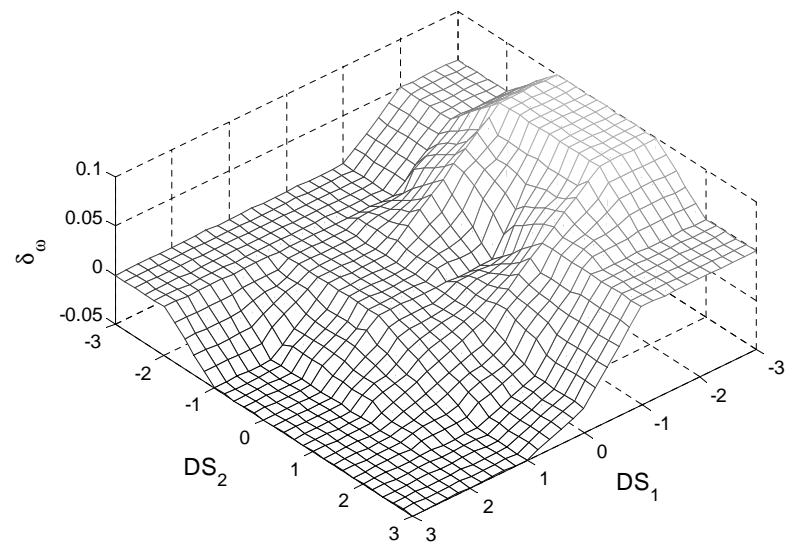

(a)

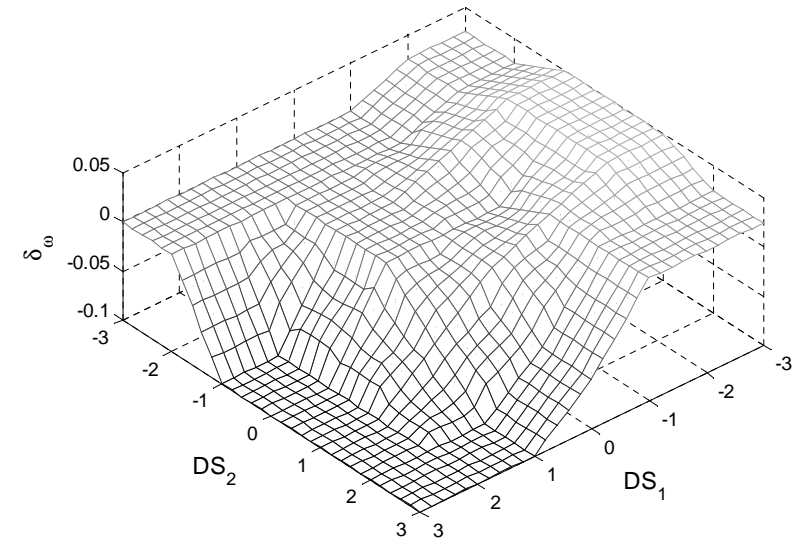

(b)

Figure 70: Control surfaces for assymetric sets (a) controller A, (b) controller B

To verify how the asymmetry in the $\delta_{\omega}$ sets affects the controller, four different initial pump speed conditions were simulated: two low initial speeds, 8 and $9 \mathrm{krpm}$, and two high initial speeds, 13 and $14 \mathrm{krpm}$. Since we are only interested in the pump speed signal, physiologic variables were not considered in this analysis. Figure 71(a) shows simulation results for $\omega_{0}=8 \mathrm{krpm}$. In this case, for controller A (solid line with circles), pump speed is increased faster at the beginning of the simulation, reaching $\omega=12 \mathrm{krpm}$ at $t=45 \mathrm{~s}$, but the transient also lasts longer. Steady state was reached only at about $t=180 \mathrm{~s}$. Pump speed for Controllers B (solid line with triangles) and C (solid black line) were similar, reaching steady state sooner, without oscillations.

Figure 71(b) shows simulation results for $\omega_{0}=9 \mathrm{krpm}$. In this case, for controller A pump speed presents oscillatory behavior, and for controllers B and C the speeds were equal. The conclusion is that a faster response is obtained when the sets of $\delta_{\omega}$ are asymmetric, allowing an increase of at most $10 \%$ in pump speed, but at the price of a more "ringing" in pump speed signal.

The simulation results for higher speeds are shown in Figures $71(\mathrm{c}, \mathrm{d})$. When $\omega_{0}=$ $13 \mathrm{krpm}$ (top panel), controller B (solid line, triangle marks) decreased pump speed faster than the other two controllers, as expected. However, pump speed was as low as $8.5 \mathrm{krpm}$, at $t=25 \mathrm{~s}$. The asymmetric definition of the output sets in controller A (solid line with circle 
marks) was responsible for the oscillatory behavior in the pump speed signal. The pump speed signal for controllers B and C (solid dark line) are quite similar when steady state is reached at $t=170$ s as shown in Figures $71(\mathrm{c}, \mathrm{d})$.

Comparing both test conditions, pump speed was less oscillatory when the initial speed was set low. However, controller B decreased pump speed more than needed. The problem with a much reduced speed is the possibility of causing sudden drops in pressure, which may cause the patient to faint. Thus, while controller $\mathrm{C}$, which has symmetric $\delta_{\omega}$ sets is more sluggish in response than the other two, it seems more suitable to handle both low and high initial pump speed conditions.
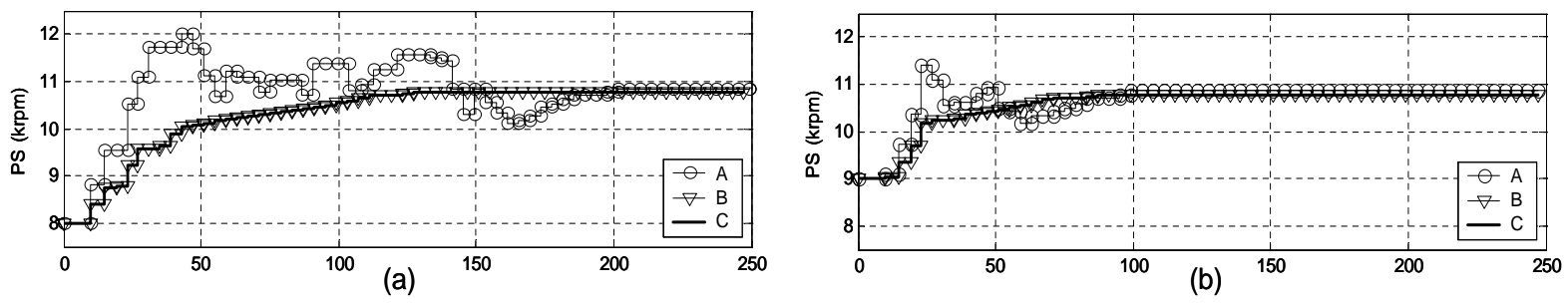

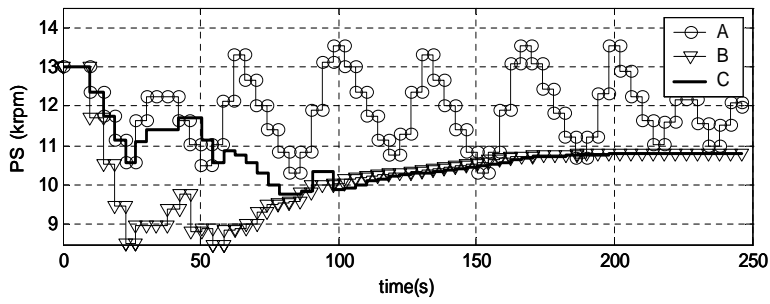

(c)

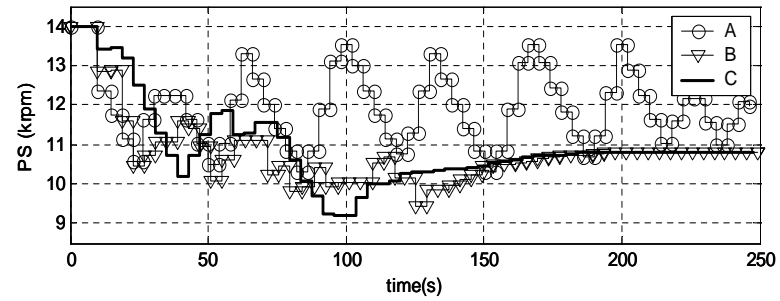

(d)

Figure 71: Pump speeds results for asymmetric sets: (a) $\omega_{0}=8 \mathrm{krpm}$, (b) $\omega_{0}=9 \mathrm{krpm}$, (c) $\omega_{0}=13 \mathrm{krpm}$ and $(\mathrm{d}) \omega_{0}=14 \mathrm{krpm}$.

\subsubsection{Final design}

In this section, the final design of the rule-based controller is presented. Based on the analysis shown in the previous sections, five membership sets were used for all variables (input and output) and the rule base included 25 rules.

6.2.3.1 Membership sets The input membership sets are defined on the range spanned by the discriminant scores $D S_{1}$ and $D S_{2}$. Three triangular membership functions were used 
for both input variables in the range $[-2,2]$, with one cross-point between consecutive sets, at the $\mu=0.5$ level. In addition, a $L(D S,-2,-1)$ function was used to deal with score values less than -2 , and a $\Gamma(D S, 1,2)$ function was used for score values greater than 2 (See Section 3.1 for the definitions of the $L$ and $\Gamma$ functions). Figure 72(a) shows the input membership sets and their respective linguistic values. The output membership sets, shown in Figure 72 (b) were defined in a similar fashion.

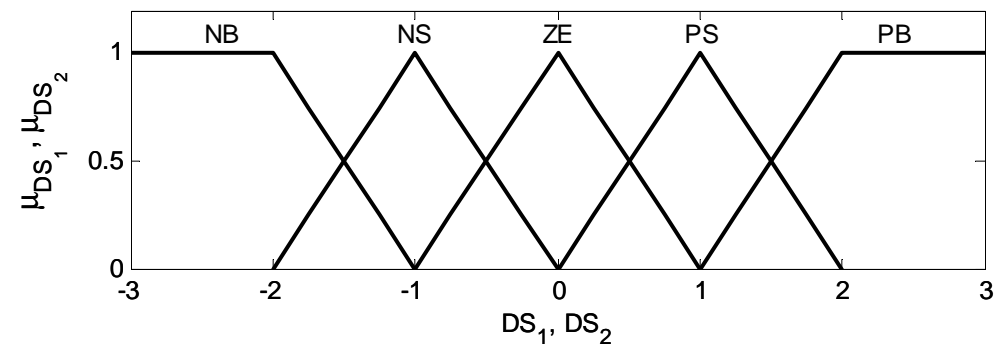

(a)

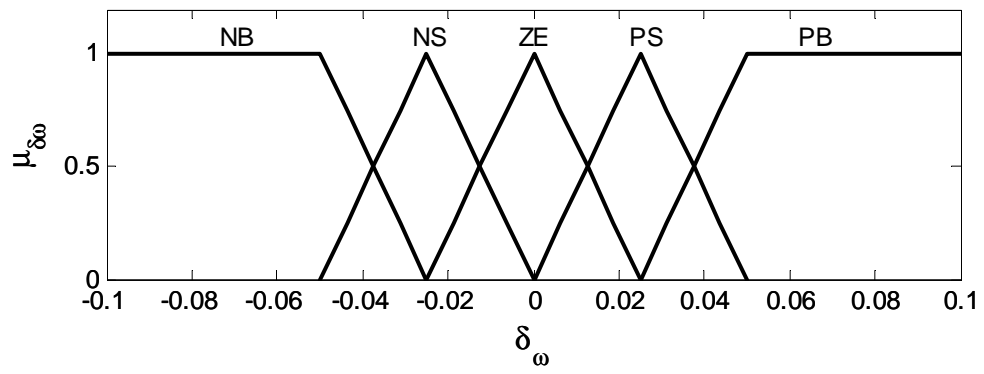

(b)

Figure 72: Controller Membership Sets. (a) Input variables; (b) Output Variables. (NB: Negative Big, NS: Negative Small, ZE: Zero, PS: Positive Small, PB: Positive Big)

6.2.3.2 Rule base The number of input fuzzy sets used dictates the number of rules in the rule-base. Since we have two input variables with 5 sets each, $5^{2}=25$ rules are needed in the rule-base. Table 16 shows the FAM bank of the controller. Considering, for instance, the first cell in that table, the following IF-THEN rule determines the output in this case:

\section{IF $D S_{1}$ is NB and $D S_{2}$ is NB THEN $\delta_{\omega}$ is $\mathrm{PS}$}

The concept behind Table 16 is to set the pump speed in such way that if severe suction (SS) is approached, pump speed should be decreased. If the suction detector indicates 
moderate suction (MS), pump speed is kept as it is. If no suction (NS) is detected, pump speed should be increased. In other words, the controller will try to keep the pump operating point in the MS region of the discriminant scores space. The parameters $\tau_{1}$ and $\tau_{2}$ should be chosen so as to avoid high amplitude variations in pump speed. A small $\delta_{\omega}$ allows "smooth" transitions in pump speed. Unless for the simulations tests shown in Section 6.3.2.1, the parameters $\tau_{1}$ and $\tau_{2}$ assume the values -0.05 and 0.05 , respectively.

Table 16: Controller Rule-base with five output sets

\begin{tabular}{|c|c|c|c|c|c|c|}
\hline & \multicolumn{5}{|c|}{$D S_{2}$} \\
\hline & & NB & NS & $\mathrm{ZE}$ & PS & $\mathrm{PB}$ \\
\hline \multirow{5}{*}{$D S_{1}$} & NB & PS & $\mathrm{PB}$ & $\mathrm{PB}$ & $\mathrm{PB}$ & PS \\
\hline & NS & $\mathrm{ZE}$ & PS & $\mathrm{ZE}$ & PS & PS \\
\hline & $\mathrm{ZE}$ & $\mathrm{ZE}$ & $\mathrm{ZE}$ & $\mathrm{ZE}$ & $\mathrm{ZE}$ & NS \\
\hline & PS & $\mathrm{ZE}$ & $\mathrm{ZE}$ & $\mathrm{ZE}$ & $\mathrm{NS}$ & NB \\
\hline & $\mathrm{PB}$ & $\mathrm{ZE}$ & $\mathrm{NB}$ & $\mathrm{NB}$ & $\mathrm{NB}$ & $\mathrm{NB}$ \\
\hline
\end{tabular}

Figure 73(a) shows the control surface of the controller. To better understand how the discriminant scores determine $\delta_{\omega}$, consider Figure $73(\mathrm{~b})$. The memberships sets of $D S_{1}$ are drawn at the right of the rule base and those of $D S_{2}$ at the top. Since the suction detector admits three possible pump states, i.e., NS (No Suction), MS (Moderate Suction) and SS (Severe Suction), the rules in the rule-base can also be divided into three groups. This is the meaning of the color code used to represent these groups: light grey cells are related to pump state NS, white cells represent MS and dark grey are related to state SS.

Because pump state NS indicates absence of suction, this means that pump speed can be increased. That is the reason why the output membership sets of light grey cells are all either PS (Positive Small) or PB (Positive Big). For instance, if $D S_{1}<-1.5$ (or if $D S_{1}$ is NB) and $D S_{2}=0$ (or if $D S_{2}$ is ZE), pump speed will be increased because $\delta_{\omega}=0.05$.

Likewise, when pump state SS is detected, pump speed should be decreased. Therefore, output membership sets of dark grey cells are all either NS (Negative Small) or NB (Negative Big). For example, if $D S_{1}>1.5$ (or if $D S_{1}$ is $\mathrm{PB}$ ) and $D S_{2}>1.5$ (or if $D S_{2}$ is $\mathrm{PB}$ ), pump speed will be decreased because $\delta_{\omega}=-0.05$. 
The white cells represent the MS pump state. In this case, $\delta_{\omega}=0$ because output membership sets are all ZE (Zero). This is the region to which the controller should drive the operating point of the pump in order to provide as much flow as possible to the patient, without inducing suction.

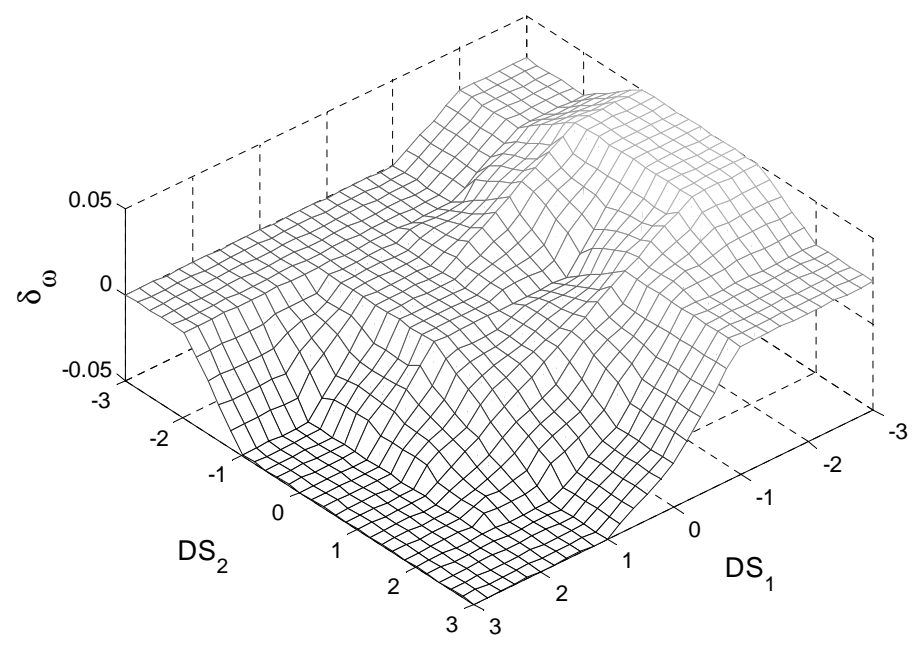

(a) control surface

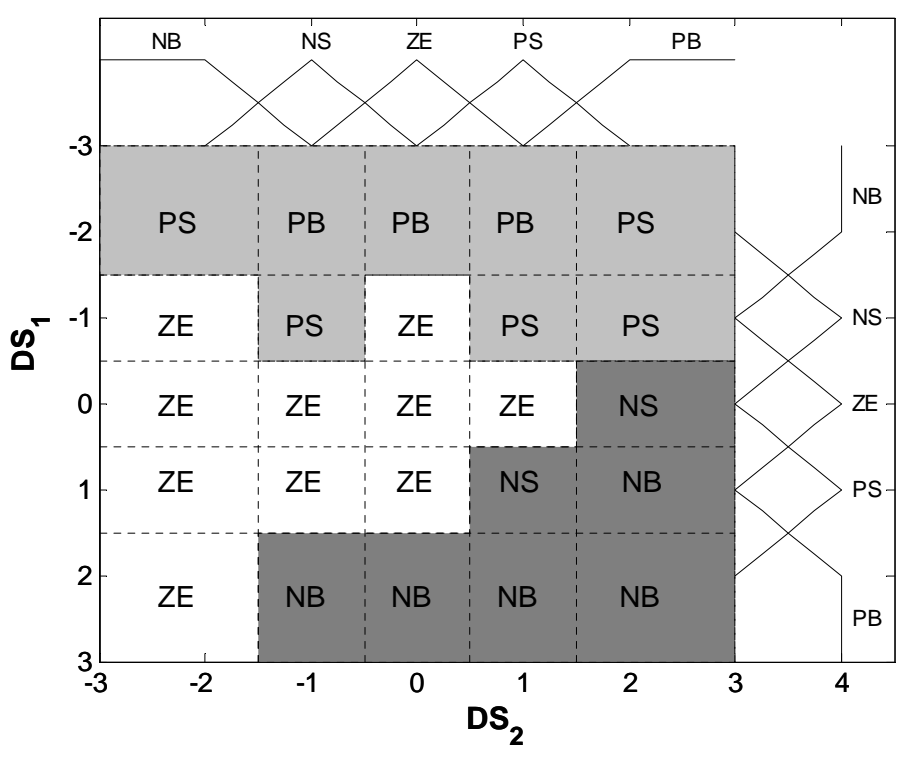

(b) partition of the input space

Figure 73: Controller transfer characteristic 


\subsection{SIMULATION STUDIES}

Simulation studies were carried out to evaluate rule-based controller performance to initial pump speed, $\omega_{0}$, physiologic parameter changes and robustness to noise. These studies are important to obtain a quantitative characterization of the interaction between the cardiovascular system, assist device and the suction detector. Since the availability for measurement and varying parameters of these various elements are limited in animal experiments, and even more so in patients, simulations are the main method of analysis and evaluation of such parameter changes [55].

To test the control system (suction detector + rule-based controller), the lumped parameter cardiovascular pump model shown in Figure 21 was used. In all of the simulations, the controller is not active during the first 10 seconds of simulation.

\subsubsection{Initial pump speed}

These tests had two goals: first, initial pump speed, $\omega_{0}$, was set low to verify if the controller could appropriately increase pump speed. Second, $\omega_{0}$ was set high to see if the controller could drive the pump out of the severe suction range. This second test can not be performed in humans, because it is dangerous for the patient's heart. In-vivo animal studies are expensive, and this kind of test can usually be done prior to terminating the animal.

Figure 74 shows two simulation results of the controller. In case (a), the initial pump speed was $\omega_{0}=8.0 \mathrm{krpm}$. As shown in the first panel of Figure 74(a), after the first 10 seconds, the controller takes action, gradually increasing pump speed. At $t=125 \mathrm{~s}$, pump speed reaches steady state at $\omega_{s s}=10.8 \mathrm{krpm}$. The second panel shows the pump flow signal. Notice that pump flow peak-to-peak amplitude decreases as pump speed increases. The last two panels show the discriminant scores and the detector classification output, respectively. The steady value of $D S_{1}$ is zero, and $D S_{2}$ is about -0.5 . These values correspond to the $Z E$ membership set in the rule base.

In Figure 74(b), the initial pump speed was $\omega_{0}=12.0 \mathrm{krpm}$. In this case, the controller

decreased pump speed, driving the pump out of the severe suction region. However, it took 
more time to reach steady state because pump speed was decreased to $\omega=10.6 \mathrm{krpm}$, around $t=25 \mathrm{~s}$ and increased to $\omega=11.5 \mathrm{krpm}$ at $t=42 \mathrm{~s}$. After that, the controller was able to decrease pump speed again and steady state was reached at $t=175 \mathrm{~s}$ and $\omega_{s s}=10.8 \mathrm{krpm}$. This behavior of the controller is due to the fact that the initial speed was set so high that moderate suction was only identified by the suction detector at $t=60 \mathrm{~s}$, as shown in the last panel of Figure 74(b). Such a high initial pump speed would not usually be encountered in human patients. 

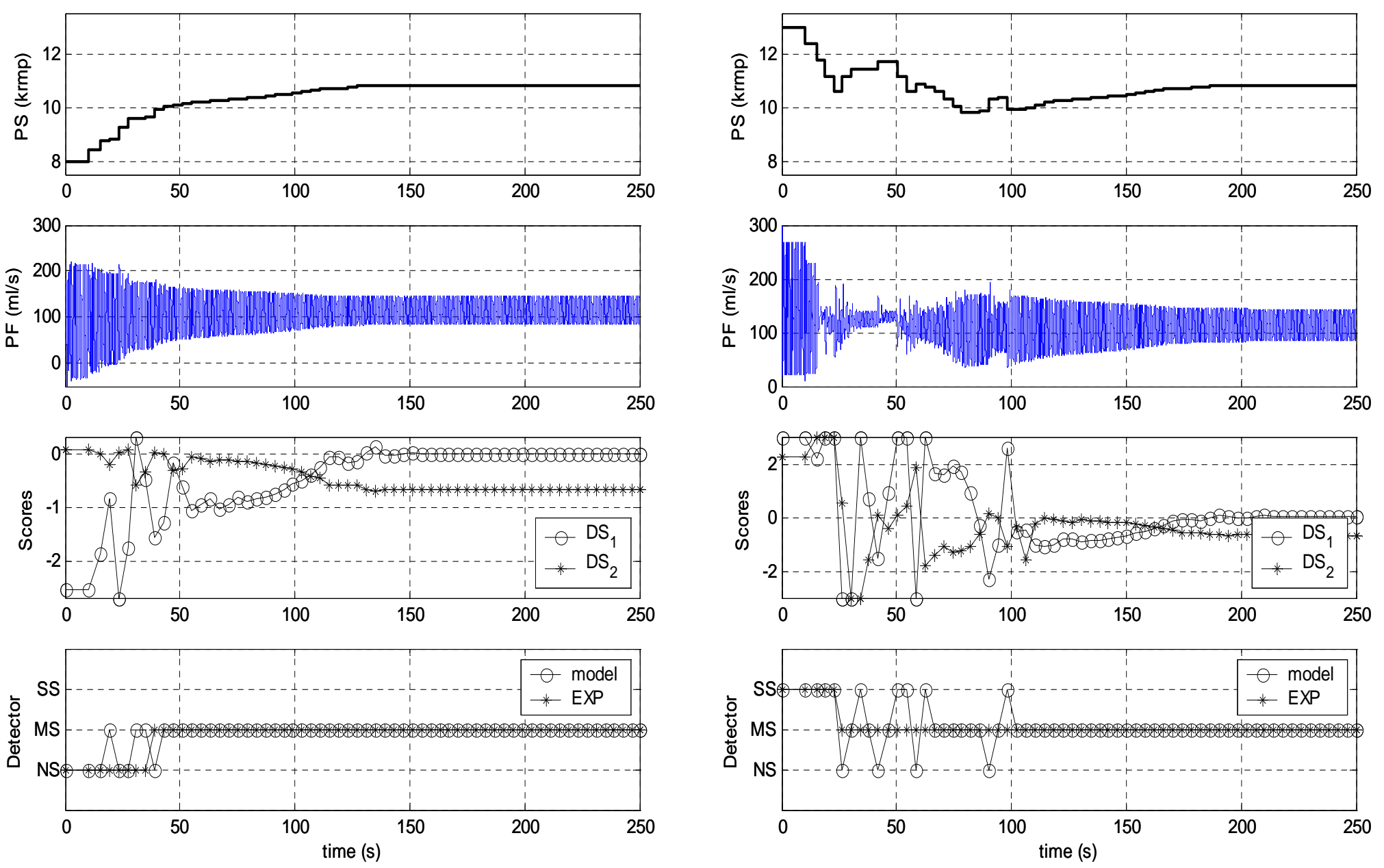

(a) Low initial speed

(b) High initial speed

Figure 74: Controller Simulation Results 
When steady state was reached in the two previous simulations, cardiac outputs were $6.7 \mathrm{l} / \mathrm{min}$ in case (a) and $6.8 \mathrm{l} / \mathrm{min}$ in case (b). These values are within the acceptable physiologic range for a normal adult person. However, besides flow, pressure perfusion has to be considered. Figure 75(a) shows that as pump speed increases, mean aortic pressure (MAP) also increases from $80 \mathrm{mmHg}$ to $122 \mathrm{mmHg}$. As for left ventricular pressure LVP, the opposite occurs, i.e., LVP decreases since the ventricle has been unloaded. When the initial speed was high, MAP was $150 \mathrm{mmHg}$. The controller reduced pump speed, and MAP reached its minimum at $105 \mathrm{mmHg}$ for $75 \leq t \leq 90$, as shown in Figure 75 (b). Finally, at the steady state condition, MAP was $122 \mathrm{mmHg}$. These results are reasonably good, even though cardiac output and MAP may be a little high because the controller tries to provide as much cardiac output as possible.

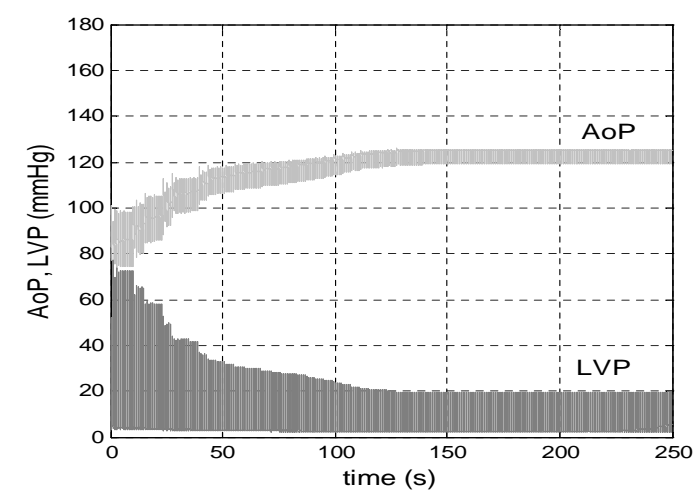

(a) Low initial speed

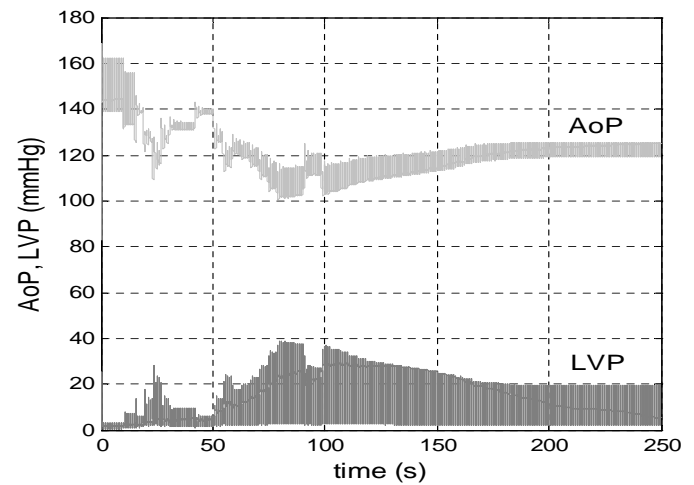

(b) High initial speed

Figure 75: Aortic and Left Ventricular Pressures

\subsubsection{Physiologic parameter change}

This section presents simulation results of the controller to physiologic parameter changes. Protocols similar to those used to test the suction detector were employed. The difference is that here the pump speed profile is not specified a priori as a ramp, but rather the rule-based controller closes the feedback loop, automatically adjusting pump speed. Table 17 shows the parameter changes studied. These parameter changes encompass preload $\left(R_{S}\right)$, heart-rate 
(HR) and contractility state $\left(E_{\max }\right)$. Resistor $R_{S}$ can have one of the following values, according to the physiologic condition to be simulated: baseline, $R_{S}=1.0 \mathrm{mmHg} . \mathrm{s} / \mathrm{ml}$; light exercising, $R_{S}=0.8 \mathrm{mmHg} . \mathrm{s} / \mathrm{ml}$; strenuous exercising, $R_{S}=0.6 \mathrm{mmHg} . \mathrm{s} / \mathrm{ml}$; and hypertension, $R_{S}=1.2 \mathrm{mmHg} . \mathrm{s} / \mathrm{ml}$.

Two case scenarios will be studied in the following section. In one, $R_{S}$ goes from baseline to light exercising and then to strenuous exercising. In the second scenario, $R_{S}$ starts at the baseline level and changes to the hypertension level. In both scenarios, step transitions from one level to another were used for $R_{S}$ and heart rate, according to Table 17. Figure 76 shows a block diagram of the entire plant, cardiovascular-pump model and the control system.

Table 17: Parameter changes to evaluate the suction detector

\begin{tabular}{|c|ll|}
\hline $\begin{array}{c}\text { CONTRACTILITY STATE } \\
\text { (relates to the native } \\
\text { heart pumping strength) }\end{array}$ & \multicolumn{1}{|c|}{$\begin{array}{c}\text { PRELOAD CONDITIONS } \\
\text { (relate to the patient's } \\
\text { level of activity) }\end{array}$} \\
\hline \hline \multirow{2}{*}{ "normal heart" } & hypertension & $R_{S}=1.2, \mathrm{HR}=75$ \\
$E_{\max }=2.0$ & baseline & $R_{S}=1.0, \mathrm{HR}=75$ \\
& light exercise & $R_{S}=0.8, \mathrm{HR}=90$ \\
& strenuous exercise & $R_{S}=0.6, \mathrm{HR}=135$ \\
\hline "sick heart" & hypertension & $R_{S}=1.2, \mathrm{HR}=75$ \\
$E_{\max }=1.0$ & baseline & $R_{S}=1.0, \mathrm{HR}=75$ \\
& light exercise & $R_{S}=0.8, \mathrm{HR}=90$ \\
& strenuous exercise & $R_{S}=0.6, \mathrm{HR}=135$ \\
\hline "very sick heart" & hypertension & $R_{S}=1.2, \mathrm{HR}=75$ \\
$E_{\max }=0.6$ & baseline & $R_{S}=1.0, \mathrm{HR}=75$ \\
& light exercise & $R_{S}=0.8, \mathrm{HR}=90$ \\
& strenuous exercise & $R_{S}=0.6, \mathrm{HR}=135$ \\
\hline
\end{tabular}

Figure 77(a) shows simulation results for the healthy heart as preload changes from baseline to heavy exercise. The initial pump speed was set to $8.0 \mathrm{krpm}$ in the first $10 \mathrm{~s}$ of simulation. After that, the feedback loop was closed and the controller updated pump 


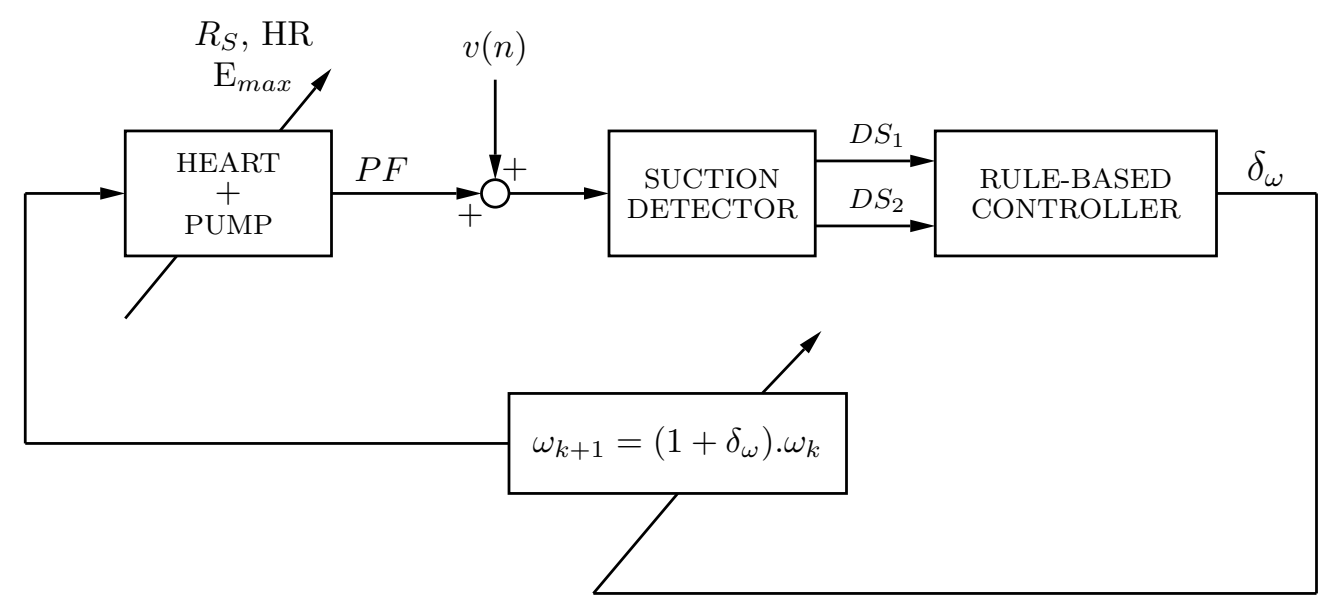

Figure 76: Block diagram of feedback control

speed. When $R_{S}$ changed from baseline to exercise, as shown in Figure 77(a) top panel, the controller increased pump speed (PS) as shown in the second panel. At $t=130$ s pump speed reached steady state at $10.9 \mathrm{krpm}$. After that, pump speed was constant regardless of the preload changes at $t=200 \mathrm{~s}$ (baseline $\rightarrow$ light exercise) and $t=400$ s (light exercise $\rightarrow$ heavy exercise). The last two panels show pump flow and pressures (AoP and LVP), respectively. Since this is a healthy heart, in the first 45s of simulation the aortic valve opened and closed because pump speed was low. After that, the aortic valve remained closed, and the VAD did all the work. When the healthy heart went from baseline to hypertension, as shown in Figure $77(\mathrm{~b})$, the controller reached steady state at $t=125 \mathrm{~s}$ and $\omega_{s s}=10.9 \mathrm{krpm}$. At $t=200 \mathrm{~s}$, preload conditions change from baseline to hypertension and pump speed stayed at about the same value.

Simulation results for the sick heart when preload changes from baseline to heavy exercise are shown in Figure 78(a). The second panel shows that pump speed increased until $10.8 \mathrm{krpm}$, prior to the transition from baseline to exercise. Minor oscillations where observed due to the preload changes. For instance, at $t=200 \mathrm{~s}$, pump speed decreased a little from $10.8 \mathrm{krpm}$ to $10.6 \mathrm{krpm}$ and at $t=400 \mathrm{~s}$ pump speed increased from $10.6 \mathrm{krpm}$ to $11.1 \mathrm{krpm}$. In both transitions the controller was able to reach steady state fairly quickly. 
In contrast to the healthy heart, the aortic valve remained closed throughout the simulation. This can be seen in the fourth panel of Figures 78(a), which shows that AoP is always greater than LVP. Moreover, the pulsatility in pump flow and pressures AoP and LVP decreases as preload changes from baseline to heavy exercise. Figure 78(b) shows the hypertension test of the sick heart. The controller increased pump speed from $10.8 \mathrm{krpm}$ to $11.0 \mathrm{krpm}$ when preload conditions change from baseline to hypertension. Because heart rate was kept constant, pump flow maximum amplitude is less than in the case of baseline to heavy exercise, and AoP amplitude is greater.

Figure 79 shows simulation results for the very-sick heart. In this case, oscillations in PS are much more pronounced than in the two previous cases. The controller was more sensitive to preload changes in both scenarios, exercising and hypertension. For instance, Figure 79(a) shows that after $t=200$ s, there were six overshoots in pump speed. When pump speed exceeded $12 \mathrm{krpm}$, severe suction occurred at $t=263,300,360,428,490 \mathrm{~s}$. This oscillatory behavior is due to the fact that the operating point kept oscillating between the NS and SS states, making the controller increase and decrease pump speed, without reaching a steady condition at the MS region in the input space. As a consequence, severe suction occurred at $t=428,490 \mathrm{~s}$. This same oscillatory behavior occurred when preload changed from baseline to hypertension as shown in Figure 79(b). One suction event occurred at $t=340$ s. Like the sick-heart, the aortic valve did not open.

Operating the pump at the moderate suction region seems reasonable for the healthy and sick hearts. No suction episodes occurred in these cases and the controller provided a steady pump speed signal. However, when the heart is very sick a more "conservative" pump speed should be used, since suction is more likely to occur due to the reduced flow. 

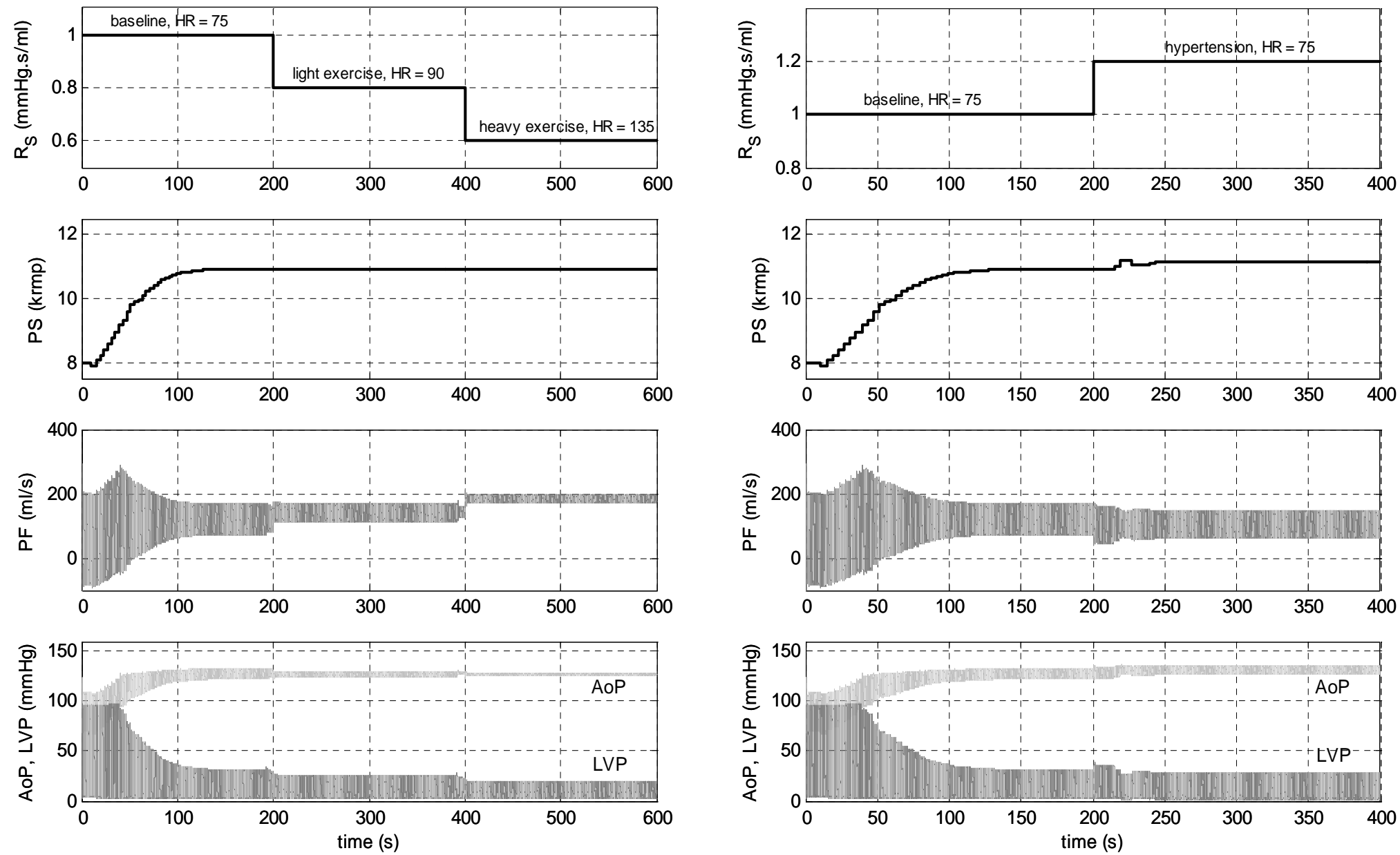

(a) baseline to exercising

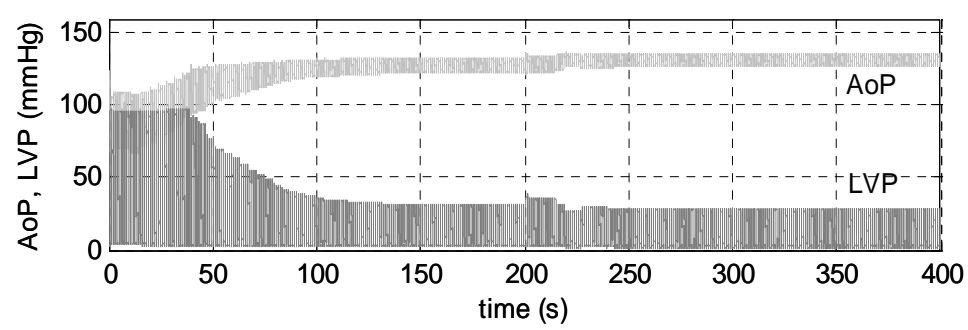

(b) baseline to hypertension

Figure 77: Controller results of Preload changes for the healthy heart $\left(E_{\max }=2.0\right)$ 

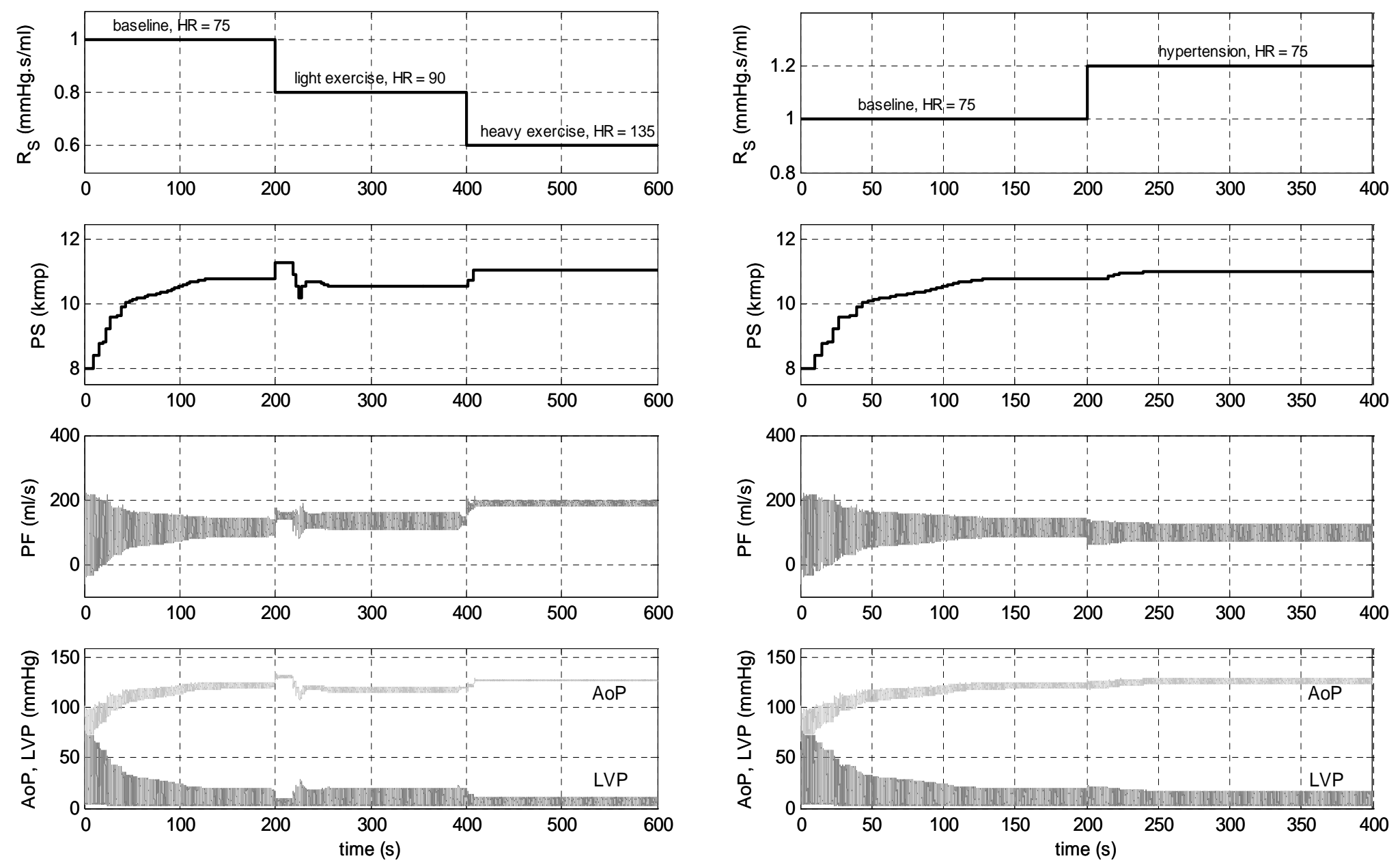

(a) baseline to exercising

(b) baseline to hypertension

Figure 78: Controller result of Preload changes for the sick heart $\left(E_{\max }=1.0\right)$ 

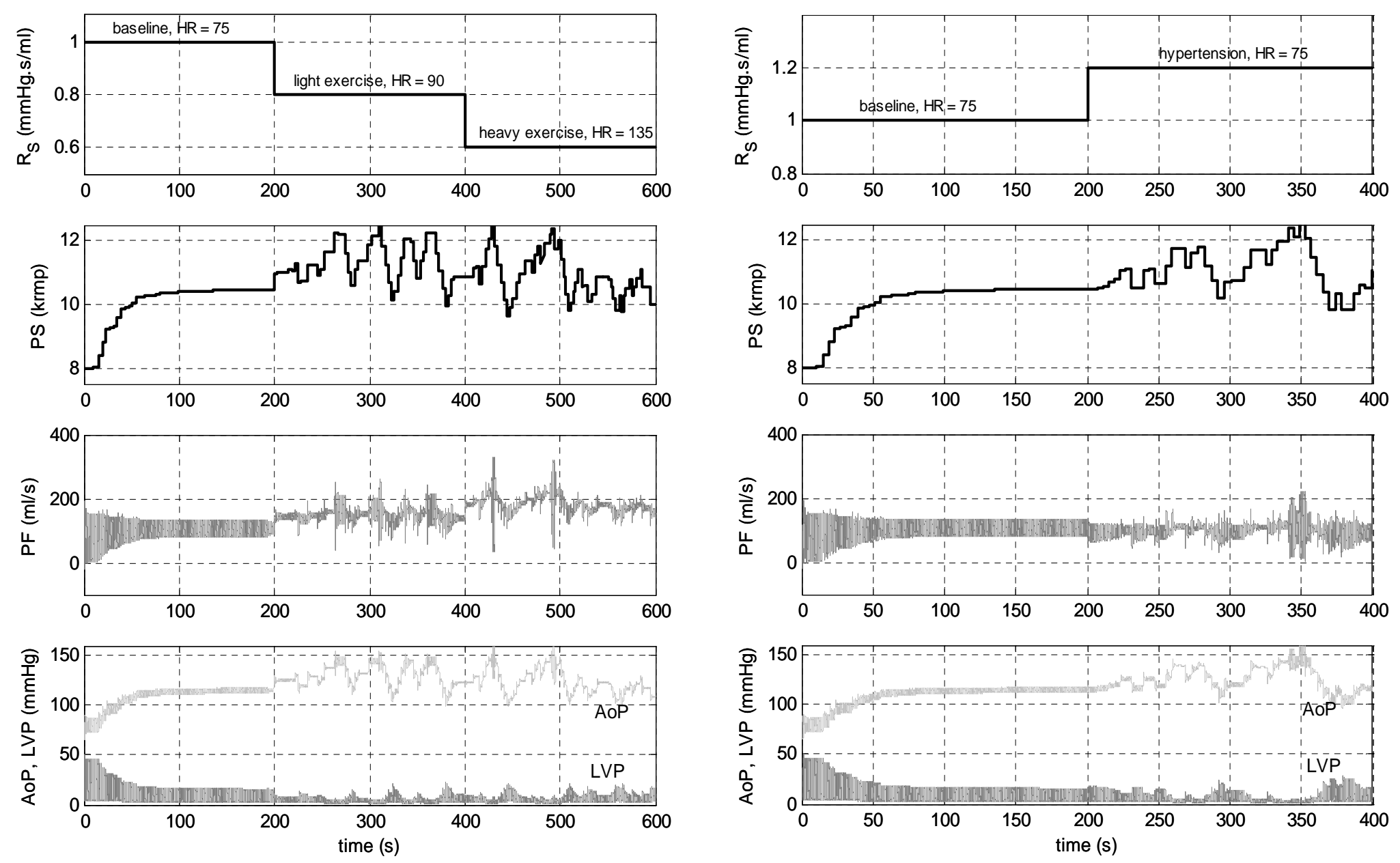

(a) baseline to exercising

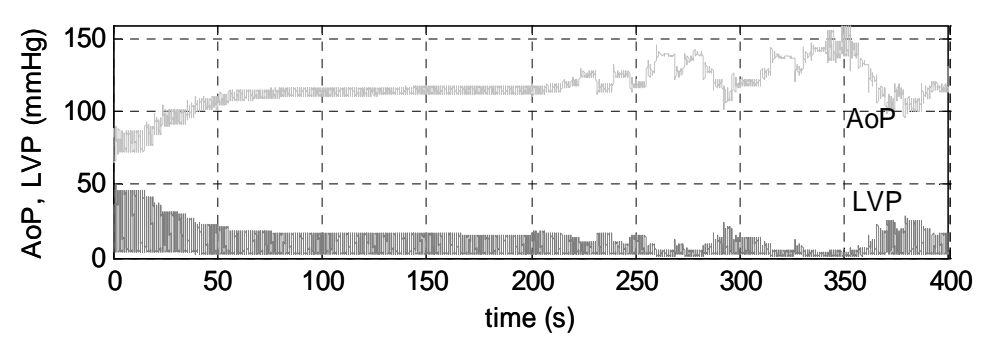

(b) baseline to hypertension

Figure 79: Controller result of Preload changes for the very sick heart $\left(E_{\max }=0.6\right)$. Note that controller fails for these test conditions. 
6.3.2.1 Tuning the controller to very sick patients As shown in Figure 79, the pump speed was oscillatory when the heart was very sick. To tune the controller to very sick patients, the designing parameters $\tau_{1}$ and $\tau_{2}$ can be modified, changing the range of the output variable, $\delta_{\omega}$. In the previous simulations, $\delta_{\omega}$ was defined in the interval $[-0.05,0.05]$.

Let $\tau_{1}=-0.02$ and $\tau_{2}=0.02$, i.e., $\delta_{\omega}$ is defined on the interval $[-0.02,0.02]$. That is, pump speed can at most be increased or decreased by $2 \%$ of its current value. Figure 80 shows the simulation results in this case. When preload conditions change from baseline to light exercise $(\mathrm{t}=200 \mathrm{~s}$, in Figure 80(a)) the controller increases pump speed from $10.4 \mathrm{krpm}$ to $10.7 \mathrm{krpm}$. The transition from light exercise to heavy exercise presented some oscillations, but the controller reached steady state at $\mathrm{t}=565 \mathrm{~s}$. No oscillations occurred for the hypertension test, as shown in Figure 80(b). These results demonstrate how the controller can be tuned to very sick patients by changing the range of the membership output sets, while keeping the sets symmetric.

A direct consequence of having pump speed being incremented or decremented by a small percent amount is an increased delay time in the controller's response. For instance, consider an initial pump speed of $\omega_{0}=8.0 \mathrm{krpm}$, when the heart is sick $\left(E_{\max }=1.0\right)$. Figure 81 shows that when $\delta_{\omega}$ is defined on the interval $[-0.05,0.05]$, the controller reaches steady state at $t=150 \mathrm{~s}$. When the range of $\delta_{\omega}$ is reduced to [-0.02, 0.02], steady state is achieved at $t=290$, which means a delay of 140 s. 

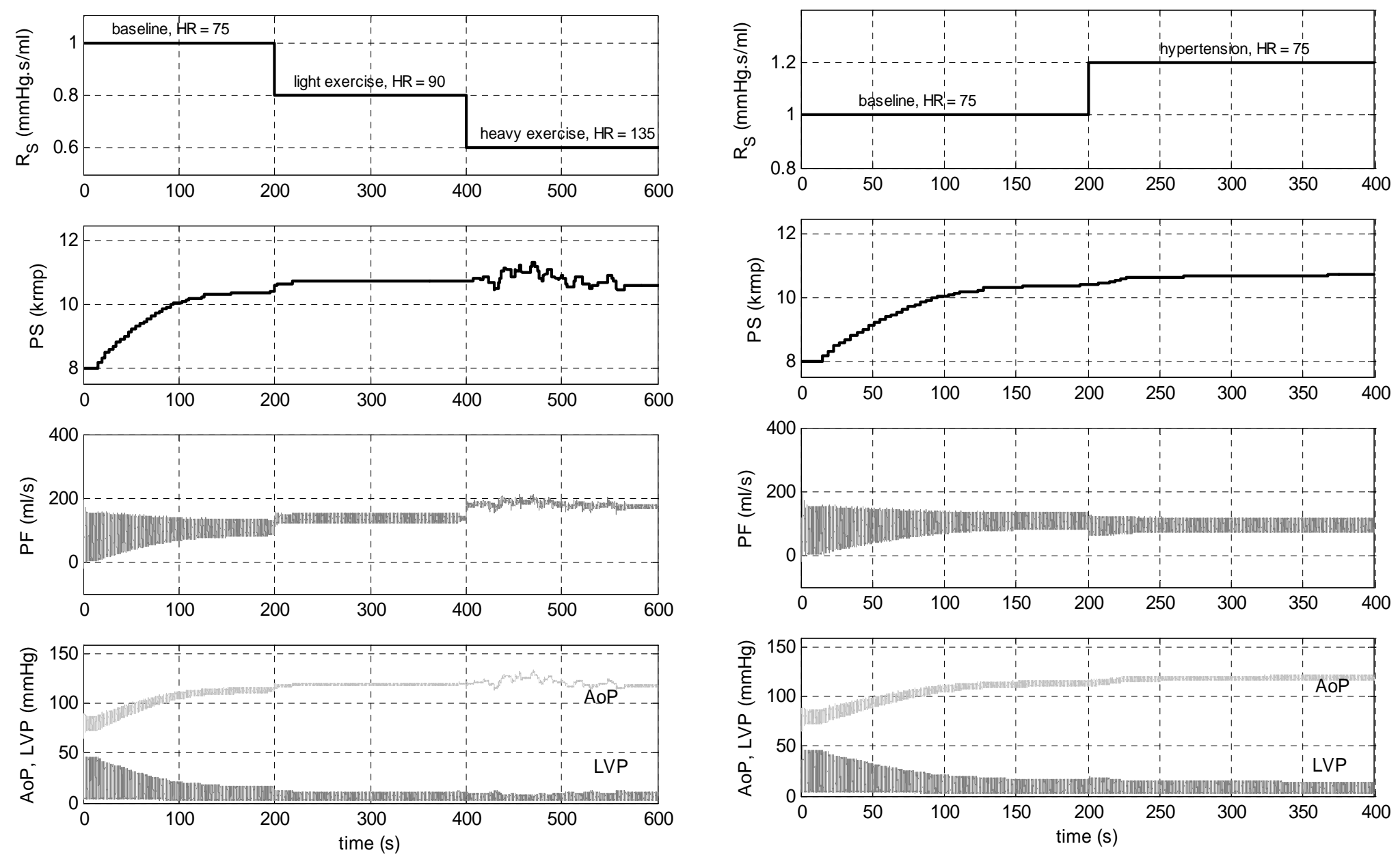

(a) baseline to exercising

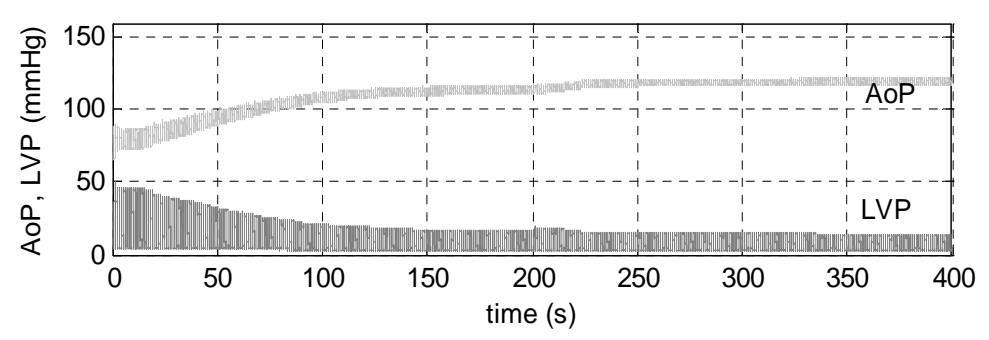

(b) baseline to hypertension

Figure 80: Controller result of Preload changes for the very sick heart $\left(E_{\text {max }}=0.6\right)$ with modified membership output sets. 


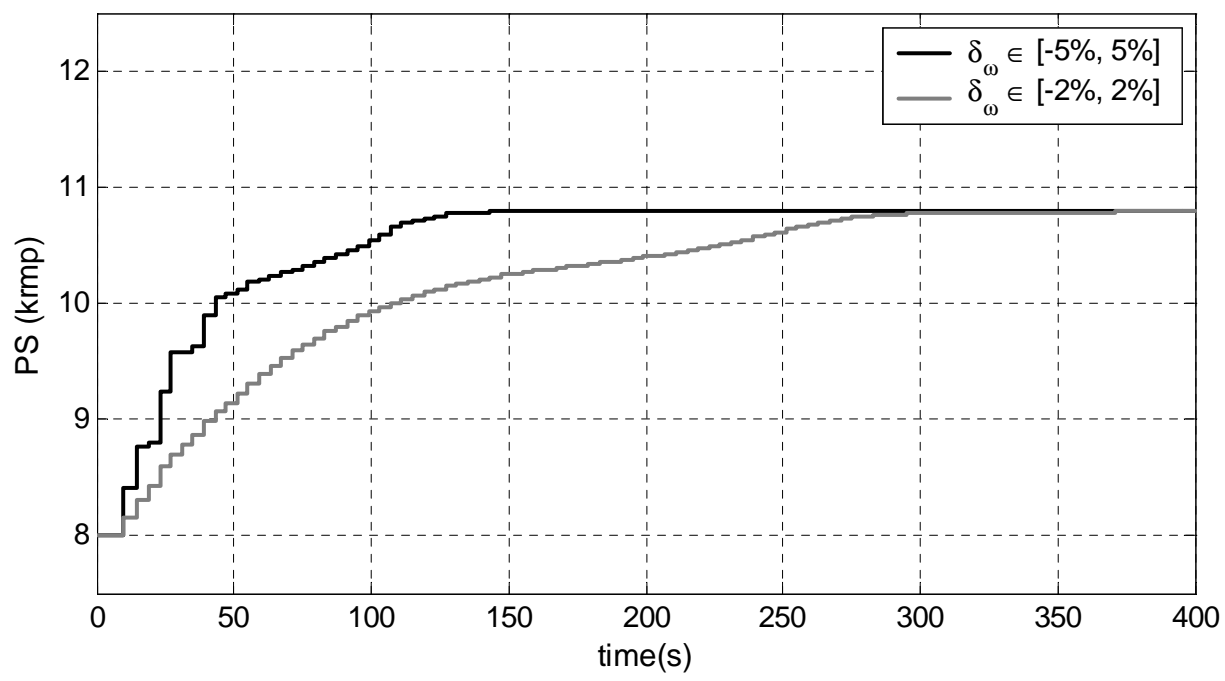

Figure 81: Delay on pump speed with modified membership output sets

\subsubsection{Hemodynamic Analysis}

In this section, the results presented in the previous section will be analyzed from a physiologic standpoint. To do so, cardiac output (CO) and mean arterial pressure (MAP) were calculated for all test conditions. Values of these two variables with (pump on) and without (pump off) ventricular assistance were calculated for comparison purposes. CO and MAP values without ventricular assistance were calculated using the cardiovascular model described in Section 4.1, under the same preload conditions shown in Table 17. The goal is to verify whether the controller has improved these hemodynamic values for the sick and very sick patients as compared with those from the healthy patient without ventricular assistance. Therefore, the hemodynamic values of the healthy heart without ventricular assistance (pump off) will be used as reference values for comparison purposes. Results for the healthy heart with ventricular assistance were included for completeness as suggested in [60].

Figure 82(a) shows the results for CO for the first simulation scenario, when preload changes from baseline to heavy exercise. The results were organized according to the activity 
states previously described. In the baseline condition $\left(R_{S}=1.0 \mathrm{mmHg} . \mathrm{s} / \mathrm{ml}, \mathrm{HR}=75 \mathrm{bpm}\right)$, the reference value for $\mathrm{CO}$ is $4.8 \mathrm{l} / \mathrm{min}$ (healthy heart without VAD). The controller was able to increase $\mathrm{CO}$ to $6.7 \mathrm{l} / \mathrm{min}$ for the sick heart, which represents a $39 \%$ increase. For the very-sick heart, a $\mathrm{CO}$ of $6.2 \mathrm{l} / \mathrm{min}$ was provided which represents a $29 \%$ increase.

In the light exercise level $\left(R_{S}=0.8 \mathrm{mmHg} . \mathrm{s} / \mathrm{ml}, \mathrm{HR}=90 \mathrm{bpm}\right)$, the reference value for $\mathrm{CO}$ is $5.9 \mathrm{l} / \mathrm{min}$. In this case, $\mathrm{CO}$ values of the sick $(7.8 \mathrm{l} / \mathrm{min})$ and very sick $(7.5 \mathrm{l} / \mathrm{min})$ hearts were increased by $32 \%$ and $27 \%$, respectively. Finally, for heavy exercise $\left(R_{S}=0.6\right.$ mmHg.s $/ \mathrm{ml}, \mathrm{HR}=135 \mathrm{bpm}$ ), the $\mathrm{CO}$ reference value is $8.0 \mathrm{l} / \mathrm{min}$. The controller provided $11.0 \mathrm{l} / \mathrm{min}$ and $9.3 \mathrm{l} / \mathrm{min}$ for the sick and very sick hearts.

Figure 82(b) compares MAP values for preload tests when SVR varies from baseline to heavy exercise. We assume again the values of the unassisted (pump off) healthy heart as reference values in each of the three physiologic states. In the baseline condition, the reference was $93 \mathrm{mmHg}$. The controller generated $122 \mathrm{mmHg}$ and $113 \mathrm{mmHg}$ for the sick and very-sick hearts, respectively. In both cases, these values were more than $20 \%$ higher than the reference value. When the hypothetic patients were exercising, the healthy person had a reference MAP of $93 \mathrm{mmHg}$, while the two patients presented MAP of $118 \mathrm{mmHg}$. Finally, when the exercise level increased to heavy, the reference MAP of the healthy person was $95 \mathrm{mmHg}$, while the sick and very-sick patients had MAP values of $127 \mathrm{mmHg}$ and $119 \mathrm{mmHg}$, respectively.

Consider now what happens when our hypothetic patients (healthy, sick, and very-sick) are hypertensive. In this case, only $R_{S}$ (SVR) changed from baseline to hypertensive level. The heart rate was constant at $75 \mathrm{bpm}$. Figure $83(\mathrm{a})$ shows that the reference value of CO was $4.8 \mathrm{l} / \mathrm{min}$ (unassisted healthy heart) in the baseline condition. The controller provided flows of $6.7 \mathrm{l} / \mathrm{min}$ and $6.2 \mathrm{l} / \mathrm{min}$ for the sick and very-sick hearts, respectively. When SVR was increased, the reference CO decreased to $4.2 \mathrm{l} / \mathrm{min}$ because heart rate was constant. The controller was able to generate $5.9 \mathrm{l} / \mathrm{min}$ and $5.1 \mathrm{l} / \mathrm{min}$ for the sick and very sick patients, respectively. The reference value of MAP shown in Figure 83(b) was 97mmHg for the hypertension state. MAP values of $126 \mathrm{mmHg}$ and $118 \mathrm{mmHg}$ were registered for the sick and very-sick hearts, respectively. 


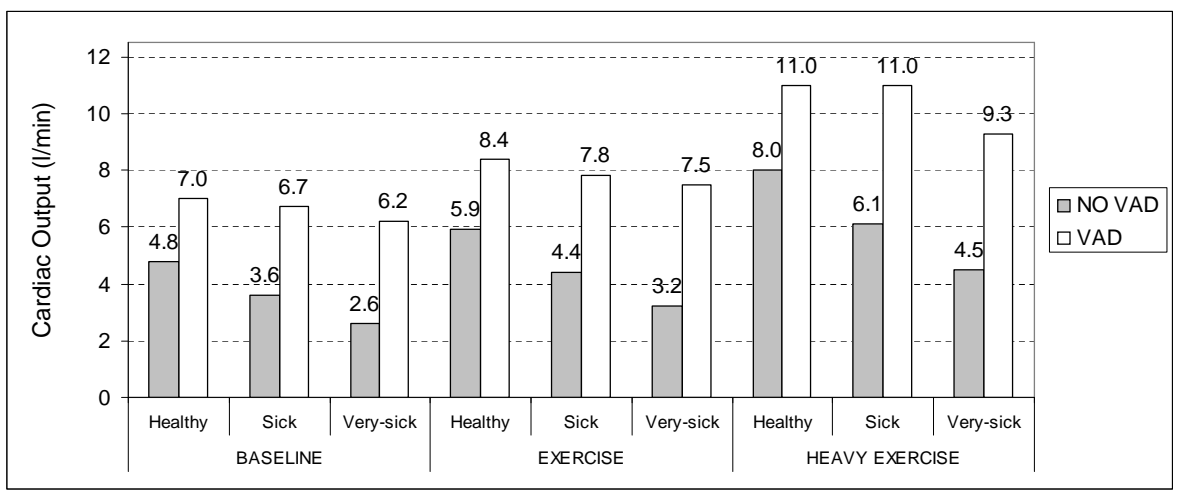

(a) Cardiac Output

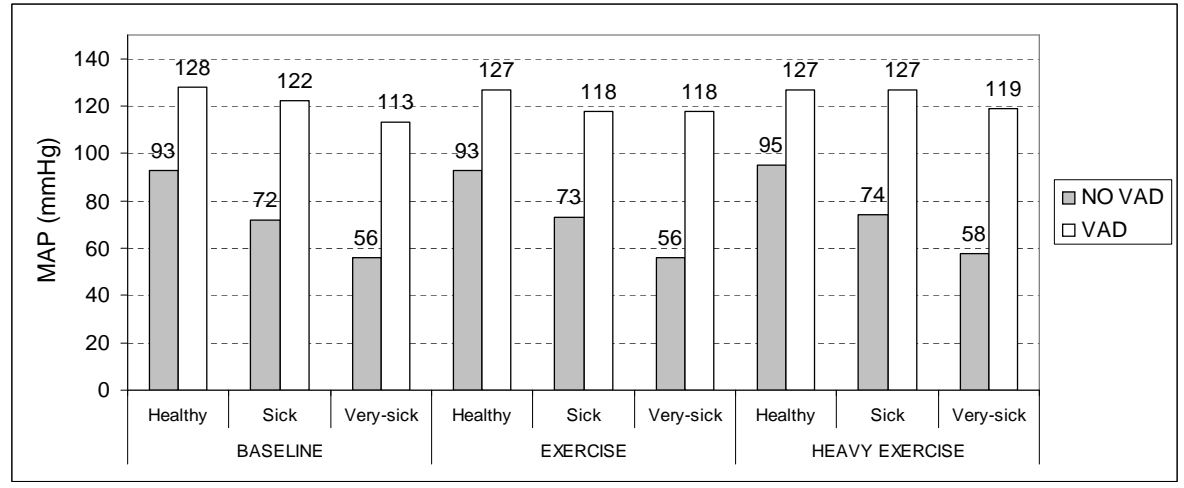

(b) Mean Arterial Pressure

Figure 82: Hemodynamic variable results for the first scenario (baseline $\rightarrow$ strenuous exercise)

The overall conclusion is that the controller is able to bring the hemodynamic parameters $\mathrm{CO}$ and MAP of the sick and very-sick hearts significantly greater than those observed in a healthy person without VAD assistance. These results also imply that the proposed controller can automatically adjust flow and pressure according to the patient's physiologic state. However, the mean arterial pressure values observed on the patients were higher than the reference values, as a result of the approach of providing as much cardiac output as possible. Moreover, the control system uses only flow information as input to drive the pump. No pressure information is taken into account in the control strategy proposed. 


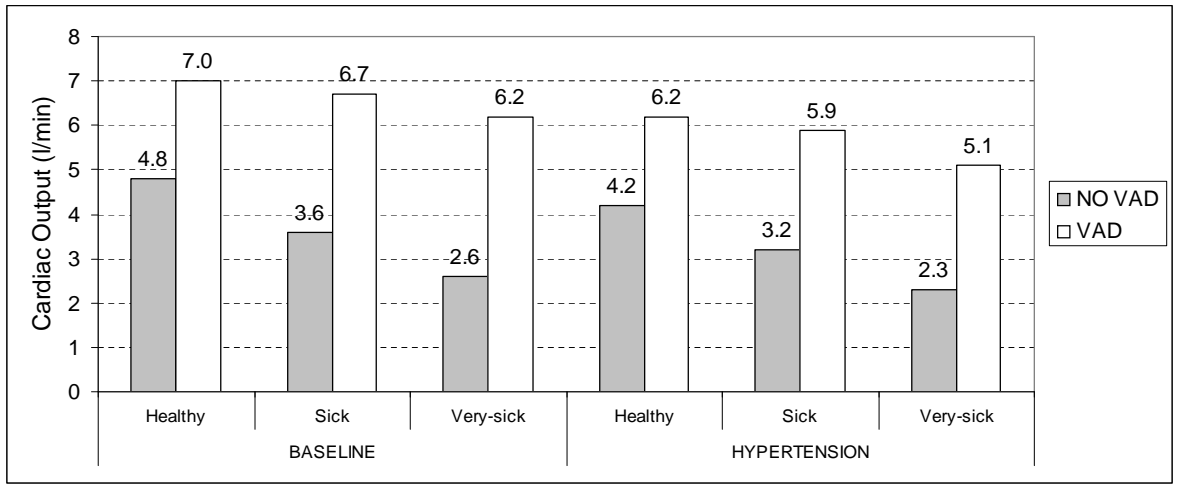

(a) Cardiac Output

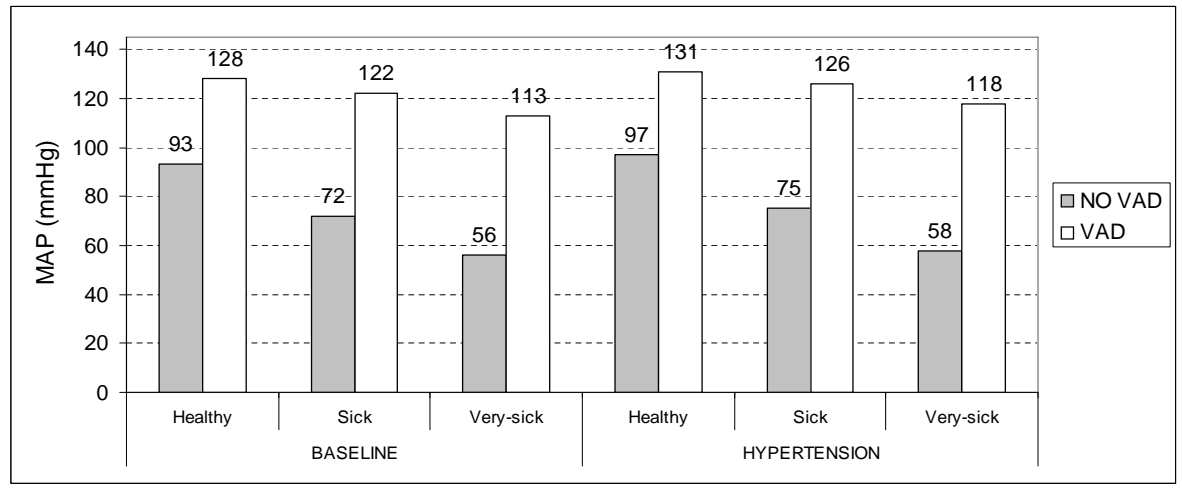

(b) Mean Arterial Pressure

Figure 83: Hemodynamic variable results for the second scenario (baseline $\rightarrow$ hypertension)

\subsubsection{Robustness to Noise}

In order to assess the controller performance when a noisy pump flow signal is presented to the suction detector input, a simulation study similar to the one shown in Section 5.4.2 was conducted. However, in this case pump speed is not defined as a ramp, but rather was adjusted by the controller. The goal here was to determine for what levels of SNR the controller is still capable of driving pump speed to an acceptable range. Tests were performed for the same three levels of contractility state as in Section 5.4.2: healthy heart $\left(E_{\max }=2.0\right)$, sick heart $\left(E_{\max }=1.0\right)$ and very sick heart $\left(E_{\max }=0.6\right)$. For each one of these contractility states, noise was added to the pump flow signal, according to the following SNR values: 
80, 60, 40, 20, 10, 5, and 2dB. Pump speed obtained without noise added to pump flow $(v(n)=0$ in Figure 76) was considered as a reference for comparison purposes. Then, the RMSE values between these reference speeds and those simulated with noise added to pump flow were calculated.

Figure 84(a) shows these errors plotted as a function of SNR when preload conditions change from baseline to strenuous exercise. For the very sick heart, the errors were calculated only for the baseline condition, since pump speed oscillated when in the exercise, strenuous exercise and hypertension preload conditions. The RMSE values for SNR higher than or equal to $20 \mathrm{~dB}$ were small, indicating that the controller's performance is not affected by these levels of SNR. At the 10bB level, these errors were 471.2, 784.3, 704.2 for the healthy, sick and very sick heart respectively. As more noise is added to the pump flow signal the RMSE keeps increasing, indicating a deterioration in the controller performance.

Figure 84(b) shows RMSE values when preload changes from baseline to hypertension. As in the previous case, SNR values higher than $20 \mathrm{~dB}$ do not compromise the performance of the controller. In addition, the healthy heart seems to be less susceptible to noise when SNR $\geq 10 \mathrm{~dB}$. However, for SNR values less than 10dB, the RMSE for the healthy heart increased more than that observed for the sick heart. For instance, at $5 \mathrm{~dB}$ the errors were 1,466.3 and 602.3 for the healthy and sick hearts respectively.

RMSE values increase as more noise is added to pump flow in all cases because the suction detector misses severe suction events due to the high noise level. This, in turn, impacts the controller's output since the discriminant scores assume values either in the MS or NS region on the control surface. Thus, pump speed will be increased, when, in reality, it should be decreased. For instance, consider Figure 85, which presents one of the simulation results for the sick heart $\mathrm{SNR}=10 \mathrm{~dB}$. Panels from the top are pump flow, pump speed, discriminant scores and the model classification result. Because pump flow was disrupted by the noise, the controller kept increasing pump speed. When $t=250 \mathrm{~s}$, the discriminant scores indicate NS, when, actually, pump flow is in the onset of severe suction. Thus, the controller increased pump speed causing the suction episode shown at $t=255 \mathrm{~s}$. Therefore, at high SNR levels severe suction events can occur. 
In conclusion, the controller can satisfactorily handle SNR levels higher than or equal to $20 \mathrm{~dB}$. This is a good result since SNR levels usually observed in flow sensors used in practice are higher than this threshold level.

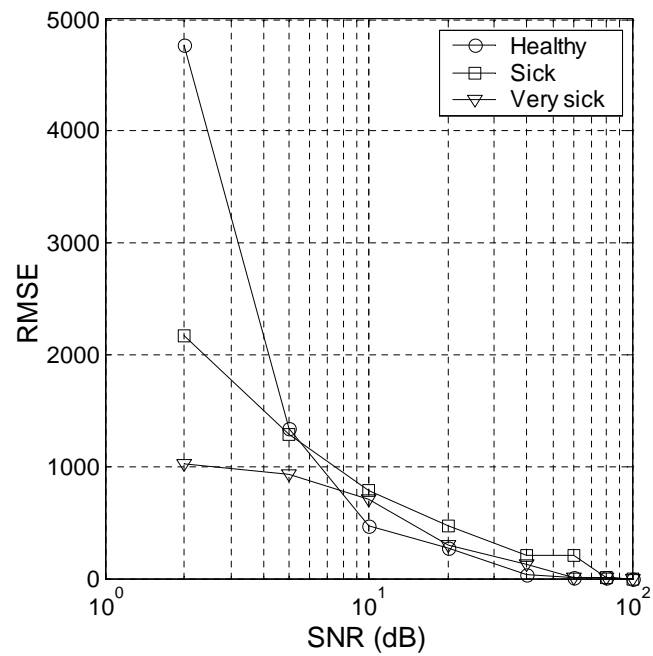

(a) scenario one, baseline to strenuous exercise

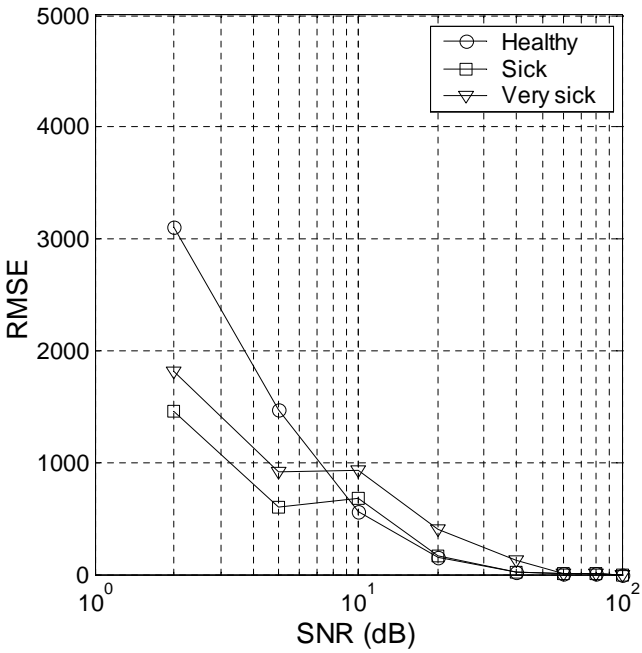

(b) scenario two, baseline to hypertension

Figure 84: RMSE as a function of SNR 

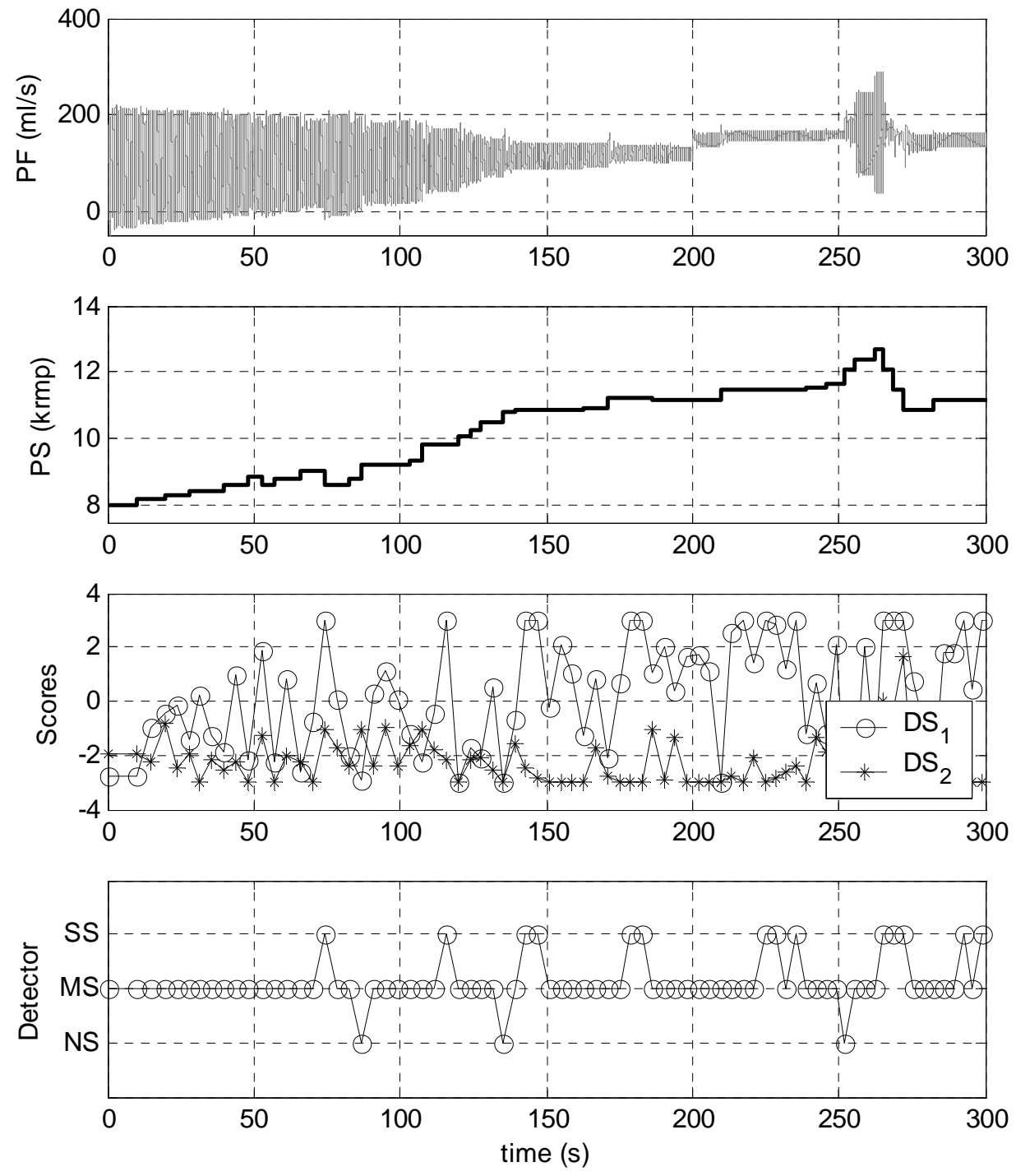

Figure 85: Example of deteriorated performance at $\mathrm{SNR}=10 \mathrm{~dB}$. Signals from the top are pump flow, pump speed, discriminant scores and the model classification result. Note that the suction event at $\mathrm{t}=250$ s was missed by the suction detector, causing the controller to erroneously increase pump speed. 


\subsection{DISCUSSION AND CONCLUSION}

A control system for rotary VADs must be safe and adaptable. Safety means that severe suction will be avoided, protecting the heart muscle. Adaptability means that the control system will automatically adjust pump speed, according to the patient's level of activity. In this work, a rule-based control system for rotary VADS was developed. This system is a combination of two main subsystems: a suction detector and a rule-based controller.

The suction detector can correctly classify pump flow patterns, based on a discriminant analysis (DA) model that combines several indices derived from the pump flow signal, to make a decision about the pump status. In this research, the pump status can be one of the following: No Suction (NS), Moderate Suction (MS) and Severe Suction (SS). The rule-based controller uses fuzzy logic to update pump speed, using information from the suction detector

- the discriminant scores, $D S_{1}$ and $D S_{2}$. The controller was designed following Mamdani's approach, which encompasses the design of the membership sets, the rule-base design and the choice of the defuzzifiation method. The transfer characteristic $u=\Phi\left(D S_{1}, D S_{2}\right)$ of the controller, also known as control surface, was used in the design phase to test several alternatives. The controller's goal is to adjust pump speed such that the operating point of the pump lies in the MS region of the discriminant scores plan.

The performance of the control system was assessed in simulations. Tests included effects of physiologic parameter changes - preload and contractility - and robustness to noise. This chapter discusses some issues regarding the cardiovascular model, the suction detection system and rule-based controller. The contributions of this work to the field and future improvements are presented in the last section. 


\subsection{CARDIOVASCULAR-PUMP MODEL}

The cardiovascular model used in this research is a uni-ventricular model. More specifically it models the left heart. A recurrent question regarding the use of cardiovascular models in this type of study is related to the order of the chosen model. Is it better to use a reduced order model or a bi-ventricular model of the cardiovascular system?

Complete models of the circulatory system allow simulation studies regarding the interaction of the low pressure circulation (right heart and lungs) and the implanted device. However, the number of parameters of the model increases and their estimation would require many invasive sensors (pressure and flow).

The cardiovascular model used in this work is of low order, when compared with complete cardiovascular models $[35,36]$. This feature along with its capabilities to reproduce hemodynamic wave forms makes it suitable for developing new control strategies for rotary blood pumps. However, the inclusion of a baroreflex in the model would better represent the interactions between the cardiovascular model and the pump. For instance, experimental data in [64] reveals a relatively constant mean aortic pressure with increasing pump speed. This effect was partially obtained in the simulations by step changes performed on systemic vascular resistance (resistor $R_{S}$ ) and heart rate (HR) in the model during the simulations. With a baroreflex control of arterial pressure added to the model, these changes would be done automatically.

\subsection{SUCTION DETECTION SYSTEM}

With regards to the design process of the suction detection system, some issues are worth

more attention, including the meaning of the prior probabilities and how to obtain severe suction patterns for training. 


\subsubsection{Meaning of the prior probabilities}

As stated in chapter 5, priors here have the meaning of relative frequency of a given group in the set. However, saying that severe suction may have the same probability of occurrence as no suction is not true. Indeed, during in-vivo trials suction is purposely caused so as to perform some physiologic test, previously defined in the experiment protocol. This probability would be even smaller with human patients, since clinicians tend to be very conservative when adjusting pump speed to protect the patient's heart.

The question then is what the priors should be, assuming that this detection system is going to be used in a patient. The answer to this question depends on several factors, such as contractility of the native heart, type of canulla, canulla position in the heart, etc. However, it seems reasonable to use equal priors to classify new pump flow samples. If more emphasis is to be given to events from a certain group, the misclassification costs can be changed to reflect the clinician's decision on that matter.

The issue of obtaining severe suction patterns is also very important. Of course, nobody would increase pump speed up to the severe suction range in a human patient. Indeed, as far as this author knows, no severe suction events in humans have been reported in the literature. The development of suction detectors for rotary blood pumps depends primarily on in-vitro (mock circulatory system) and in-vivo animal experiments. This situation persists because of the difficulty in having human data available to design suction detectors. As reported in [67], there is a difference in the behavior of the native heart when comparing animal and human subjects. The animals usually have relatively healthy cardiac function while human implanted patients are suffering from left ventricular failure. Therefore, animal hemodynamic variables and pump signals (current, speed, etc) are expected to be different from those observed in humans.

This fact does not invalidate the suction detector proposed in this research. If human data were available, the detector could have been developed using the same approach. Based on the experimental results presented in Section 5.3, the detector can be used with in vivo animal experiments. To use the detector in human clinical trials it is necessary to derive discriminant functions based on human data. 


\subsubsection{Comparison with current technology}

The accuracy of the suction detection system presented in this research was primarily compared with the proportional chance criteria. This is the common test performed when dealing with discriminant analysis. It is expected that a DA classifier should perform better than chance.

It is also important to compare the proposed model with current suction detectors recently described in the literature. In this regard, there are two particular suction detectors of interest for comparison purposes: the suction detection proposed by the Vienna group and the one developed at the University of New South Wales (NSW), Australia.

The Vienna group's $[11,65]$ suction detector is based on several time-based indices extracted from the pump flow waveform. Using a window length of 5 seconds, patterns extracted from the pump flow waveform are compared against snapshots of pump flow previously stored and classified in a data base by human experts, in order to decide whether suction is present. The authors acknowledge that the proposed method can easily increase exponentially the possible combinations in its optimization procedure, if applied to a multiple-beat analysis. This might be an issue for real time applications of the proposed method. This system has been tested using 1000 records of approximately 100 patients. Each record contains 5s of pump flow, current and arterial pressure.

The NSW group $[66,67]$ proposed a non-invasive suction detection system that extracts several indices from the pump speed signal, using a 6 seconds long time window. Using a binary decision tree algorithm (CART), predictions of new membership cases are made, presenting high accuracy rate, in the two-group classification problem, i.e., when one needs to decide between suction and no suction. This system has been validated using ex vivo porcine experiments.

Because the pump states are different among these approaches, as shown in Table 18, a comparison between those approaches can only be made for the two-group classification problem.

The meaning of the pump states proposed by the researchers from Vienna is self-explanatory. To reduce their original 5 groups to only two groups, they define flow patterns Types 
Table 18: Pump states

\begin{tabular}{lll}
\hline Vienna's group & NSW group & Pitt/CMU group \\
\hline Type 1: Certainly no suction & PR: Regurgitant flow & NS: No Suction \\
Type 2: Most probably no suction & VE: Ventricular ejection & MS: Moderate Suction \\
Type 3: Undecided & ANO: Nonopening aortic valve & SS: Severe Suction \\
Type 4: Most probably suction & PVC-I: Partial collapse internitent & \\
Type 5: Suction & PVC-C: Partial collapse continuous & \\
\hline
\end{tabular}

1 and 2 as No Suction (NS) and patterns Types 4 and 5 as Suction (S).

The researchers from NSW say that the PR (Regurgitant flow) state occurs when pump speed is so low that negative (regurgitant) pump flow occurs during diastole. VE (ventricular ejection) is typified by ventricular ejection in systole, i.e., the aortic valve still opens and closes as expected. State ANO occurs when the aortic valve remains closed and maximum LVP is less than AoP. At this state, ventricular contractions may cease if pump speed is increased and pump hemodynamics is largely influenced by the respiratory system. In State PVC-I the influence of the respiration on the cardiac behavior often causes partial collapse of the ventricle to occur intermittently, that is, not every heart beat but over a fraction of the respiratory cycle. State PVC-C is exhibited when a suction event occurs every cardiac cycle. The NSW group combines pumping states VE and ANO to form the No Suction (NS) state; states PVC-I and PVC-C are combined to form the Suction (S) state. Table 19 shows a comparison between these suction detectors. The parameters used to compare those strategies are input variable used, sensitivity, specificity, invasive sensor, patient independence (a calibration task based on each patient's condition should not be required).

It is not clear whether the groups from NSW and Vienna have divided their data bases into two sets (training and test) as was done in this research. Accuracy results that take into account training samples tend to be positively biased. Therefore, such results should be carefully analyzed to avoid optimistic conclusions about the detector classification precision. 
One advantage that the NSW group has over the others is the fact that their approach is non-invasive. This means that they actually do not need implanted sensors to detect suction. Both the Vienna and NSW groups intend to develop a physiologic controller in which their suction detectors will be part of a feedback control loop, but such control systems have not been reported yet.

Based on the comparison shown in Table 19, the Pitt/CMU approach has results comparable to the other two groups in the two group classification problem.

Table 19: Comparison between the three suction detectors

\begin{tabular}{|c|c|c|c|c|c|}
\cline { 2 - 6 } \multicolumn{1}{c|}{} & $\begin{array}{c}\text { Input } \\
\text { Variable }\end{array}$ & $\begin{array}{c}\text { Sensitivity } \\
(\%)\end{array}$ & $\begin{array}{c}\text { Specificity } \\
(\%)\end{array}$ & $\begin{array}{c}\text { Invasive } \\
\text { sensor }\end{array}$ & $\begin{array}{c}\text { Patient } \\
\text { Independence }\end{array}$ \\
\hline $\begin{array}{c}\text { Vienna's } \\
\text { group }\end{array}$ & $\begin{array}{c}\text { Pump } \\
\text { flow }\end{array}$ & 99 & 100 & yes & no \\
\hline NSW & Pump & 99.5 & 100 & no & yes \\
group & speed & 93 & 100 & yes & yes \\
\hline $\begin{array}{c}\text { Pitt/CMU } \\
\text { Training }\end{array}$ & $\begin{array}{c}\text { Pump } \\
\text { flow }\end{array}$ & 96 & 97 & yes & yes \\
\hline $\begin{array}{c}\text { Pitt/CMU } \\
\text { Test }\end{array}$ & $\begin{array}{c}\text { Pump } \\
\text { flow }\end{array}$ & & & & \\
\hline
\end{tabular}




\subsection{RULE-BASED CONTROLLER}

Fuzzy controllers are usually practically constructed, instead of theoretically designed, using some expert knowledge and computer simulations. They have been applied in many engineering fields, presenting satisfactory results. However, expert knowledge is usually difficult to translate in a mathematical analytic form, which makes the analysis and design of such non-linear controllers a difficult task.

In this research, basic "guidelines" commonly used in the literature were followed. For instance, the number of input variables was two, like in many applications of Mamdani's type fuzzy controllers. Five symmetric triangular membership sets were used both for input and output variables. A rule base with 25 rules was designed and a computationally simple defuzzification method was chosen.

The design of the rule base is perhaps the most important design task, since the rule base dictates the output of the controller. Notice that in the control system shown in Chapter 6, the inputs are not states of the plant (which is the cardiovascular-pump model) but rather, the discriminant scores from the suction detector. This fact actually helped in the rule base design, making it more systematic, because we could associate pump states (NS, MS, SS) with output membership sets (PB or PS; ZE; NB or NS).

The designed controller provides one answer for the problem of updating pump speed in a rotary VAD, but it may not be the definitive answer. There may be other designs that can generate similar or even better results. However, the controller presented here demonstrates the feasibility of this approach to drive the pump in the MS region as planned. Fuzzy logic can provide a mechanism to combine the two discriminant scores so that they can be used in the control scheme. Moreover, the controller can be tuned to a given patient, which is a desirable characteristic.

\subsubsection{Tuning of Controller}

Suction patterns depend not only on pump speed and canulla position. They also depend on the patient. Characteristics such as contractility state, size of the heart, level of activity and 
mood are likely to influence the occurrence of suction. Therefore, any control application should allow tuning of the controller, making the controller patient specific. In our approach, the controller can be tuned by changing the output membership definitions.

Changes in the output sets of the controller were analyzed in Section 6.2.2. As stated there, it is possible to make the controller increase or decrease pump speed faster by changing the output membership set definitions. Thus, in a future hardware implementation of that system, an option in the interface system might be available to allow the clinician to perform such adjustments on the controller settings.

Tuning was necessary because the very sick heart was more sensitive to pump speeds on the MS range. However, the approach of driving the pump in this range in order to provide as much cardiac output as possible has the undesirable effect of increasing mean aortic pressure. Physiologically, a possible application of such an approach would be with patients that need ventricular unloading to promote ventricular recovery. As reported in [68], ventricular unloading and augmentation of aortic flow are a novel therapy to treat congestive heart failure patients. Since the control approach proposed here promotes ventricular unloading, consequently increasing flow through the aorta, patients may benefit from the proposed control system developed in this research.

Regurgitant flow patterns were not considered in the control system designed in this research because preventing suction was the main goal. Since regurgitation usually occurs at low pump speeds, the physiologic consequences of a reduced speed are regurgitation (backflow) and a drop in perfusion pressure. To avoid this problem, a lower speed bound, say $\omega_{\text {min }}$ could be set to prevent backflow. Each new pump speed value, $\omega_{k+1}$, can be compared with that minimum admissible threshold and not be allowed to drop below it.

\subsubsection{Emergency mode}

Like any other engineering design, this control system may fail. Under this circumstance, the control system has to prevent harmful conditions to the patient, avoiding suction and yet providing adequate blood flow into the circulatory system. 
We do not expect for this control system (detector and controller) alone to be responsible for the entire operation of the pump. Indeed, as suggested in $[4,56]$, a supervisory system is needed to assure that system faults are promptly detected, shifting the operating mode to a safe default mode. System faults can include sensor failures, software failure, or uncertainty concerning the reliability of the control actions or functioning of the assist device itself. Thus, the default mode should provide a constant pump speed that is low enough to avoid suction, while sustaining a nominal flow output.

\subsection{CONTRIBUTIONS AND FUTURE WORK}

The main contributions of this work to the field are:

a) A rule-based control system for rotary VADs was developed. This system can automatically adjust pump speed while keeping cardiac output and mean arterial pressure within acceptable physiologic ranges and avoiding suction;

b) The cardiovascular model used here was developed and validated. Its reduced order makes it suitable for developing control strategies for VADs.

c) The time-frequency based index, which is the standard deviation of the instantaneous mean frequency of pump flow, is the first application of a time-frequency technique to the suction detection problem in rotary VADs;

d) This is the first time that a window whose duration depends on a certain number of heart beats has been used in suction detection;

e) The Discriminant Analysis has been applied to the suction detection problem;

f) Membership functions of the rule-based controller output can be changed, allowing fine tuning of the control system;

The proposed control system may be improved by using non-invasive information in the suction detector to eliminate the need for implanted sensors that have reliability issues for

long term implants. This can be done by developing new discriminant functions using pump motor control signals, such as pump current, pump speed or motor voltage. For certain types 
of VADs (e.g. WorldHeart LVAD) the use of the rotor position signal would be beneficial, since this signal is highly correlated with pump inlet pressure. Moreover, estimation of pump flow would be another alternative.

Rotary VADs have also been used as recovery therapy in certain cases. As the pump performs most of the work load, being responsible for cardiac output and perfusion pressure of the circulation, the heart may gradually recover from its illness, improving its contractility state. A control strategy for rotary VADs should take this healing process into account. For instance, if the heart recovers, pump speed should not be kept at high values, close to the onset of severe suction because this represents extreme ventricular unloading. This, in turn, will keep left ventricular pressure always below aortic pressure and, as a consequence, the aortic valve will remain closed. Hence, stiffness of the valve may occur, which is a deleterious condition. Therefore, it is important to have also a monitoring system that can recognize improvements in the contractility state of the heart, so as to decrease pump speed gradually, allowing the heart to eject blood through the aortic valve.

The next step towards the development of a viable control system to be used with human patients is its validation in vivo. In vivo animal experiments are necessary to verify to what extent the results obtained in simulations studies agree with experimental results. Of particular interest are the control system performance when the heart is very weak, its adaptability to different physiologic conditions and robustness to noise.

\subsection{CONCLUSION}

A control system for rotary blood pumps was presented. That system is a combination of two main subsystems: a suction detector and a rule-based controller. The suction detector can correctly classify pump flow patterns, using a discriminant analysis (DA) model that combines several indices derived from the pump flow signal to make a decision about the pump status. The pump status can be one of the following: No Suction (NS), Moderate Suction (MS) and Severe Suction (SS). 
The discriminant scores, which are the output of the suction detector, were used as inputs to a rule-based controller. Based on this information, the controller updates pump speed, providing adequate flow and pressure perfusion to the patient. Both subsystems were tested under different preload conditions (baseline, light exercise, strenuous exercise and hypertension) and contractility states (healthy, sick and very sick) presenting satisfactory results for the healthy and sick hearts. In these two cases, the control system was able to automatically adjust pump speed, providing pump flow according to the patient's level of activity, while sustaining adequate perfusion pressures and avoiding suction. However, mean arterial pressure was high, since the controller provided large cardiac output values. Additionally, no pressure information is considered in the proposed control approach. The very sick heart is more sensitive to speeds on the MS range, which may cause the occurrence of suction. In this case, pump speed should be carefully updated. In Section 6.3.2.1, it was shown that by changing the design parameters $\tau_{1}$ and $\tau_{2}$ in order to decrease the range spanned by the membership output sets, it is possible to drive the operating point to a steady state condition without introducing suction.

The control system performance was not adversely affected by noise until SNR was less than $20 \mathrm{~dB}$, which is a higher noise level than is commonly encountered in flow sensors used clinically for this type of application. For SNR levels less than this value, the controller tends to increase pump speed because the noise disrupts the detector performance, causing it to miss severe suction cases and to increase pump speed. 
APPENDIX A

\section{STATE EQUATIONS FOR THE CARDIOVASCULAR MODEL}

Isovolumic Phases: $\boldsymbol{r}(x)=\left[\begin{array}{l}0 \\ 0\end{array}\right]$

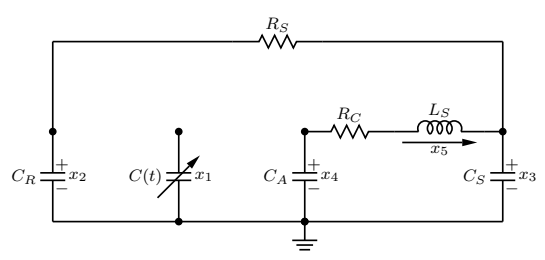

$$
\dot{\boldsymbol{x}}=\left[\begin{array}{ccccc}
\frac{-\dot{C}(t)}{C(t)} & 0 & 0 & 0 & 0 \\
0 & \frac{-1}{R_{S} C_{R}} & \frac{1}{R_{S} C_{R}} & 0 & 0 \\
0 & \frac{1}{R_{S} C_{S}} & \frac{-1}{R_{S} C_{S}} & \frac{1}{C_{S}} & 0 \\
0 & 0 & 0 & 0 & \frac{-1}{C_{A}} \\
0 & 0 & \frac{-1}{L_{S}} & \frac{1}{L_{S}} & \frac{-R_{C}}{L_{S}}
\end{array}\right] x
$$

Ejection Phase: $\boldsymbol{r}(x)=\left[\begin{array}{c}0 \\ \frac{\boldsymbol{r}\left(x_{1}-x_{4}\right)}{R_{A}}\end{array}\right]$

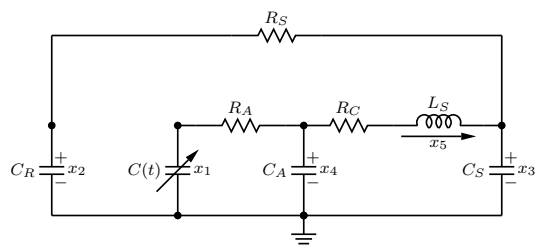

$$
\dot{\boldsymbol{x}}=\left[\begin{array}{ccccc}
\frac{-\dot{C}(t)}{C(t)}-\frac{1}{C(t) R_{A}} & 0 & 0 & \frac{1}{C(t) R_{A}} & 0 \\
0 & \frac{-1}{C_{R} R_{S}} & \frac{1}{C_{R} R_{S}} & 0 & 0 \\
0 & \frac{1}{C_{S} R_{S}} & \frac{-1}{C_{S} R_{S}} & 0 & \frac{1}{C_{S}} \\
\frac{1}{C_{A} R_{A}} & 0 & 0 & \frac{-1}{C_{A} R_{A}} & \frac{-1}{C_{A}} \\
0 & 0 & \frac{-1}{L_{S}} & \frac{1}{L_{S}} & \frac{-R_{C}}{L_{S}}
\end{array}\right] \boldsymbol{x}
$$


Filling Phase: $\boldsymbol{r}(x)=\left[\begin{array}{c}\frac{\boldsymbol{r}\left(x_{2}-x_{1}\right)}{R_{M}} \\ 0\end{array}\right]$

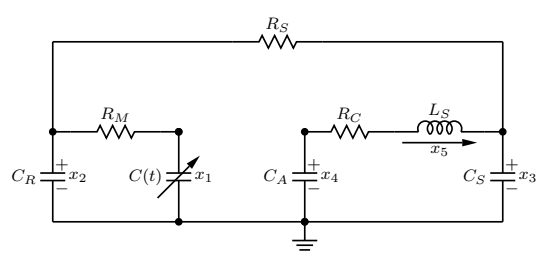

$\dot{\boldsymbol{x}}=\left[\begin{array}{ccccc}\frac{-\dot{C}(t)}{C(t)}-\frac{1}{C(t) R_{M}} & \frac{1}{C(t) R_{M}} & 0 & 0 & 0 \\ \frac{1}{C_{R} R_{M}} & \frac{-1}{C_{R}}\left(\frac{1}{R_{M}}+\frac{1}{R_{S}}\right) & \frac{1}{C_{R} R_{S}} & 0 & 0 \\ 0 & \frac{1}{C_{S} R_{S}} & \frac{-1}{C_{S} R_{S}} & 0 & \frac{1}{C_{S}} \\ 0 & 0 & 0 & 0 & \frac{-1}{C_{A}} \\ 0 & 0 & \frac{-1}{L_{S}} & \frac{1}{L_{S}} & \frac{-R_{C}}{L_{S}}\end{array}\right] \boldsymbol{x}$


APPENDIX B

STATE EQUATIONS FOR THE CARDIOVASCULAR-PUMP MODEL

Isovolumic Phases: $\boldsymbol{r}(x)=\left[\begin{array}{l}0 \\ 0\end{array}\right]$

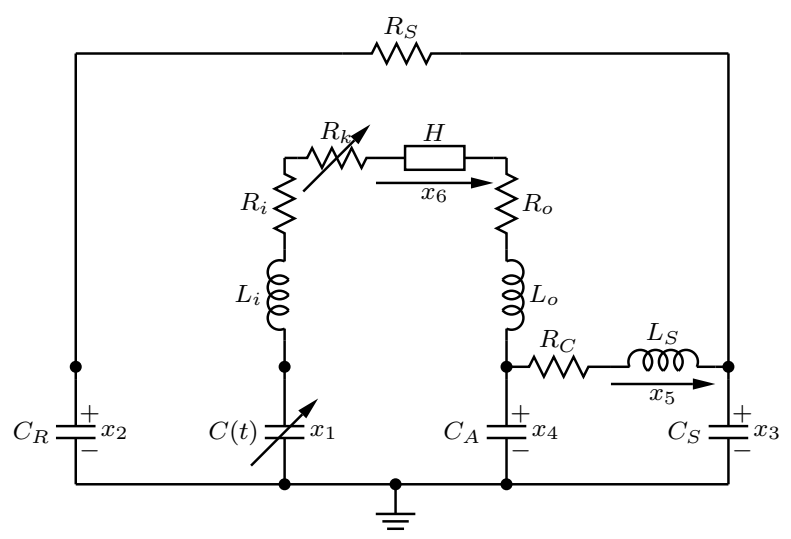

$$
\dot{\boldsymbol{x}}=\left[\begin{array}{cccccc}
\frac{-\dot{C}(t)}{C(t)} & 0 & 0 & 0 & 0 & \frac{-1}{C(t)} \\
0 & \frac{-1}{R_{S} C_{R}} & \frac{1}{R_{S} C_{R}} & 0 & 0 & 0 \\
0 & \frac{1}{R_{S} C_{S}} & \frac{-1}{R_{S} C_{S}} & \frac{1}{C_{S}} & 0 & 0 \\
0 & 0 & 0 & 0 & \frac{-1}{C_{A}} & \frac{1}{C_{A}} \\
0 & 0 & \frac{-1}{L_{S}} & \frac{1}{L_{S}} & \frac{-R_{C}}{L_{S}} & 0 \\
\frac{1}{L^{*}} & 0 & 0 & \frac{-1}{L^{*}} & 0 & \frac{-R^{*}}{L^{*}}
\end{array}\right] \boldsymbol{x}+\left[\begin{array}{c}
0 \\
0 \\
0 \\
0 \\
0 \\
\frac{-\beta_{2}}{L^{*}}
\end{array}\right] u(t)
$$


Ejection Phase: $\boldsymbol{r}(x)=\left[\begin{array}{c}0 \\ \frac{r\left(x_{1}-x_{4}\right)}{R_{A}}\end{array}\right]$

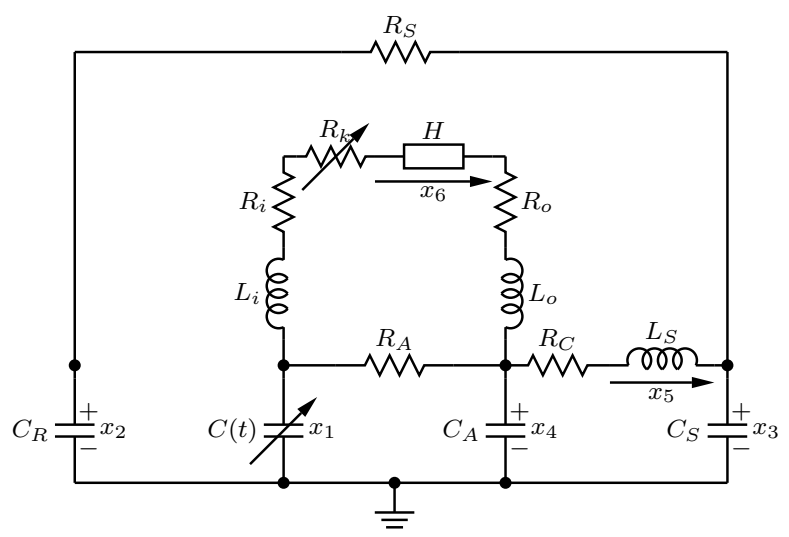

$$
\dot{\boldsymbol{x}}=\left[\begin{array}{cccccc}
\frac{-\dot{C}(t)}{C(t)}-\frac{1}{C(t) R_{A}} & 0 & 0 & \frac{1}{C(t) R_{A}} & 0 & \frac{-1}{C(t)} \\
0 & \frac{-1}{R_{S} C_{R}} & \frac{1}{R_{S} C_{R}} & 0 & 0 & 0 \\
0 & \frac{1}{R_{S} C_{S}} & \frac{-1}{R_{S} C_{S}} & \frac{1}{C_{S}} & 0 & 0 \\
\frac{1}{C_{A} R_{A}} & 0 & 0 & \frac{-1}{C_{A} R_{A}} & \frac{-1}{C_{A}} & \frac{1}{C_{A}} \\
0 & 0 & \frac{-1}{L_{S}} & \frac{1}{L_{S}} & \frac{-R_{C}}{L_{S}} & 0 \\
\frac{1}{L^{*}} & 0 & 0 & \frac{-1}{L^{*}} & 0 & \frac{-R^{*}}{L^{*}}
\end{array}\right] \boldsymbol{x}+\left[\begin{array}{c}
0 \\
0 \\
0 \\
0 \\
0 \\
\frac{-\beta_{2}}{L^{*}}
\end{array}\right] u(t)
$$


Filling Phase: $\boldsymbol{r}(x)=\left[\begin{array}{c}\frac{\boldsymbol{r}\left(x_{2}-x_{1}\right)}{R_{M}} \\ 0\end{array}\right]$

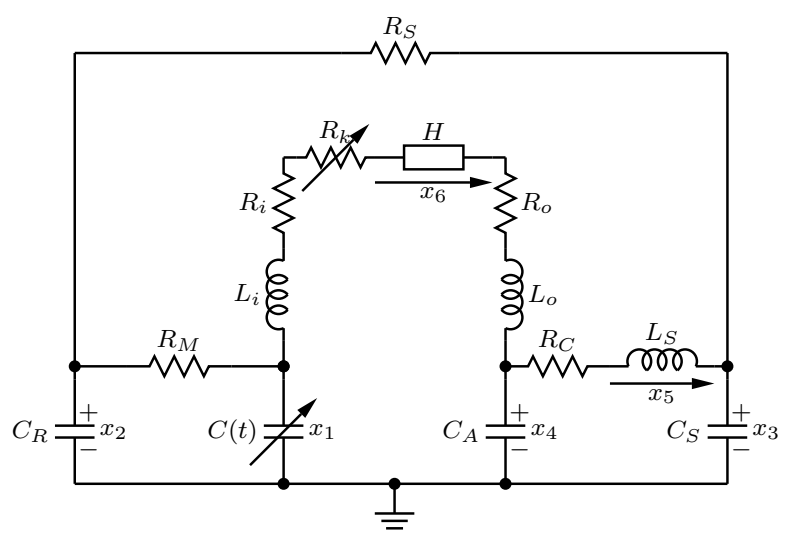

$\dot{\boldsymbol{x}}=\left[\begin{array}{cccccc}\frac{-\dot{C}(t)}{C(t)}-\frac{1}{C(t) R_{M}} & \frac{1}{C(t) R_{M}} & 0 & 0 & 0 & \frac{-1}{C(t)} \\ \frac{1}{C_{R} R_{M}} & \frac{-1}{C_{R}}\left(\frac{1}{R_{M}}+\frac{1}{R_{S}}\right) & \frac{1}{R_{S} C_{R}} & 0 & 0 & 0 \\ 0 & \frac{1}{R_{S} C_{S}} & \frac{-1}{R_{S} C_{S}} & \frac{1}{C_{S}} & 0 & 0 \\ 0 & 0 & 0 & 0 & \frac{-1}{C_{A}} & \frac{1}{C_{A}} \\ 0 & 0 & \frac{-1}{L_{S}} & \frac{1}{L_{S}} & \frac{-R_{C}}{L_{S}} & 0 \\ \frac{1}{L^{*}} & 0 & 0 & \frac{-1}{L^{*}} & 0 & \frac{-R^{*}}{L^{*}}\end{array}\right] \boldsymbol{x}+\left[\begin{array}{c}0 \\ 0 \\ 0 \\ 0 \\ 0 \\ \frac{-\beta_{2}}{L^{*}}\end{array}\right] u(t)$ 
APPENDIX C

\section{CONFUSION MATRICES - SVR TESTS}

\section{Healthy heart}

$R_{S}=1.0, \mathrm{HR}=75 \mathrm{bpm}$

\begin{tabular}{c|cccc} 
& NS & MS & SS & Total \\
\hline NS & 19 & 0 & 0 & 19 \\
MS & 4 & 2 & 0 & 6 \\
SS & 0 & 2 & 13 & 15
\end{tabular}

$R_{S}=0.6, \mathrm{HR}=135 \mathrm{bpm}$

\begin{tabular}{c|cccc} 
& NS & MS & SS & Total \\
\hline NS & 30 & 6 & 0 & 36 \\
MS & 1 & 15 & 0 & 16 \\
SS & 0 & 2 & 21 & 23
\end{tabular}

$R_{S}=0.8, \mathrm{HR}=90 \mathrm{bpm}$

\begin{tabular}{c|cccc} 
& NS & MS & SS & Total \\
\hline NS & 26 & 2 & 0 & 28 \\
MS & 1 & 9 & 0 & 10 \\
SS & 0 & 3 & 18 & 21
\end{tabular}

$R_{S}=1.2, \mathrm{HR}=75 \mathrm{bpm}$

\begin{tabular}{c|cccc} 
& NS & MS & SS & Total \\
\hline NS & 25 & 0 & 0 & 25 \\
MS & 3 & 3 & 0 & 6 \\
SS & 0 & 4 & 15 & 19
\end{tabular}


Sick heart

$R_{S}=1.0, \mathrm{HR}=75 \mathrm{bpm}$

\begin{tabular}{c|cccc} 
& NS & MS & SS & Total \\
\hline NS & 19 & 0 & 0 & 19 \\
MS & 2 & 4 & 0 & 6 \\
SS & 0 & 2 & 13 & 15
\end{tabular}

$R_{S}=0.6, \mathrm{HR}=135 \mathrm{bpm}$

\begin{tabular}{c|cccc} 
& NS & MS & SS & Total \\
\hline NS & 30 & 0 & 0 & 30 \\
MS & 4 & 11 & 1 & 16 \\
SS & 0 & 2 & 25 & 27
\end{tabular}

\section{Very Sick heart}

$R_{S}=1.0, \mathrm{HR}=75 \mathrm{bpm}$

\begin{tabular}{c|cccc} 
& NS & MS & SS & Total \\
\hline NS & 19 & 0 & 0 & 19 \\
MS & 2 & 4 & 0 & 6 \\
SS & 0 & 3 & 12 & 15
\end{tabular}

$R_{S}=0.6, \mathrm{HR}=135 \mathrm{bpm}$

\begin{tabular}{c|cccc} 
& NS & MS & SS & Total \\
\hline NS & 29 & 1 & 0 & 30 \\
MS & 5 & 7 & 2 & 14 \\
SS & 0 & 0 & 27 & 27
\end{tabular}

$R_{S}=0.8, \mathrm{HR}=90 \mathrm{bpm}$

\begin{tabular}{c|cccc} 
& NS & MS & SS & Total \\
\hline NS & 25 & 4 & 0 & 29 \\
MS & 0 & 8 & 0 & 8 \\
SS & 0 & 5 & 17 & 22
\end{tabular}

$R_{S}=1.2, \mathrm{HR}=75 \mathrm{bpm}$

\begin{tabular}{c|cccc} 
& NS & MS & SS & Total \\
\hline NS & 23 & 1 & 0 & 24 \\
MS & 2 & 5 & 0 & 7 \\
SS & 0 & 3 & 15 & 18
\end{tabular}

$R_{S}=0.8, \mathrm{HR}=90 \mathrm{bpm}$

\begin{tabular}{c|cccc} 
& NS & MS & SS & Total \\
\hline NS & 23 & 5 & 0 & 28 \\
MS & 0 & 8 & 1 & 9 \\
SS & 0 & 4 & 17 & 21
\end{tabular}

$R_{S}=1.2, \mathrm{HR}=75 \mathrm{bpm}$

\begin{tabular}{c|cccc} 
& NS & MS & SS & Total \\
\hline NS & 22 & 2 & 0 & 24 \\
MS & 2 & 5 & 0 & 7 \\
SS & 0 & 3 & 15 & 18
\end{tabular}


APPENDIX D

\section{FAM BANKS OF CONTROL SURFACES}

a) Using three output sets

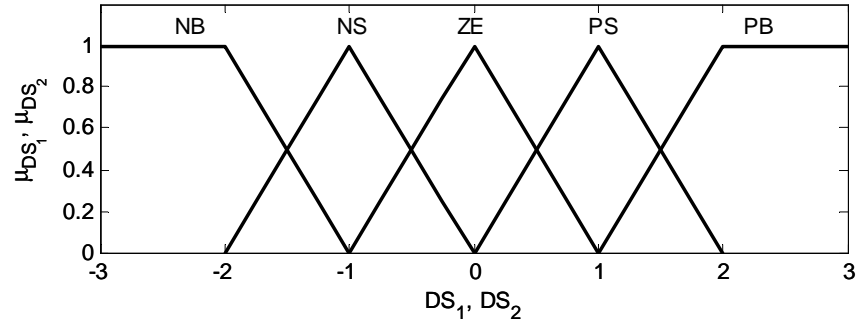

(a)

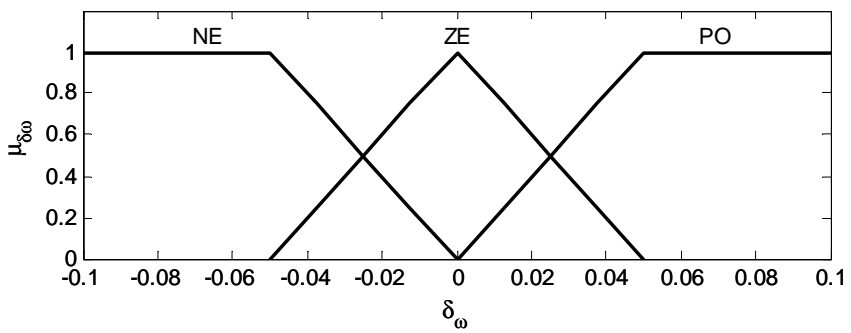

(b)

Controller Membership Sets. (a) Input variables; (b) Output Variable. (NB: Negative Big, NS: Negative Small, ZE: Zero, PS: Positive Small, PB: Positive Big, PO: Positive, NE: Negative) 
Table 20: Controller Rule-base for three output sets

\begin{tabular}{|c|c|c|c|c|c|c|}
\hline & \multicolumn{5}{|c|}{$D S_{2}$} \\
\hline & & NB & NS & $\mathrm{ZE}$ & PS & $\mathrm{PB}$ \\
\hline \multirow{5}{*}{$D S_{1}$} & NB & $\mathrm{PO}$ & $\mathrm{PO}$ & $\mathrm{PO}$ & $\mathrm{PO}$ & $\mathrm{PO}$ \\
\hline & NS & $\mathrm{ZE}$ & $\mathrm{PO}$ & $\mathrm{ZE}$ & $\mathrm{PO}$ & $\mathrm{PO}$ \\
\hline & $\mathrm{ZE}$ & $\mathrm{ZE}$ & $\mathrm{ZE}$ & $\mathrm{ZE}$ & $\mathrm{ZE}$ & $\mathrm{NE}$ \\
\hline & PS & $\mathrm{ZE}$ & $\mathrm{ZE}$ & $\mathrm{ZE}$ & $\mathrm{NE}$ & $\mathrm{NE}$ \\
\hline & $\mathrm{PB}$ & $\mathrm{ZE}$ & $\mathrm{NE}$ & $\mathrm{NE}$ & $\mathrm{NE}$ & $\mathrm{NE}$ \\
\hline
\end{tabular}


b) Using five output sets

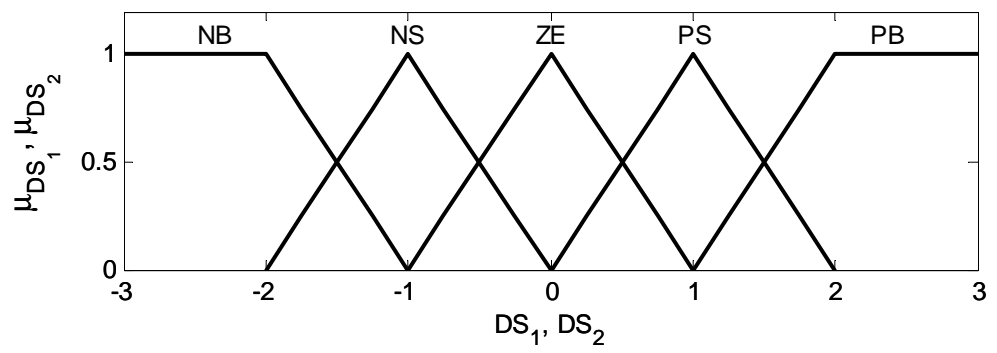

(a)

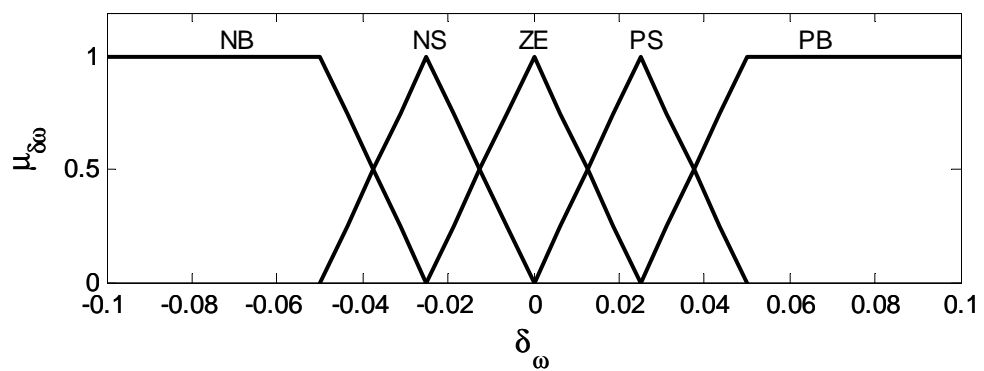

(b)

Controller Membership Sets. (a) Input variables; (b) Output Variables. (NB: Negative Big, NS:

Negative Small, ZE: Zero, PS: Positive Small, PB: Positive Big)

Table 21: Controller Rule-base for five output sets

\begin{tabular}{|c|c|c|c|c|c|c|}
\hline & \multicolumn{5}{|c|}{$D S_{2}$} \\
\hline & & NB & NS & $\mathrm{ZE}$ & PS & $\mathrm{PB}$ \\
\hline \multirow{5}{*}{$D S_{1}$} & NB & PS & $\mathrm{PB}$ & $\mathrm{PB}$ & $\mathrm{PB}$ & PS \\
\hline & NS & $\mathrm{ZE}$ & PS & $\mathrm{ZE}$ & PS & PS \\
\hline & $\mathrm{ZE}$ & $\mathrm{ZE}$ & $\mathrm{ZE}$ & $\mathrm{ZE}$ & $\mathrm{ZE}$ & $\mathrm{NS}$ \\
\hline & PS & $\mathrm{ZE}$ & $\mathrm{ZE}$ & $\mathrm{ZE}$ & NS & $\mathrm{NB}$ \\
\hline & PB & $\mathrm{ZE}$ & $\mathrm{NB}$ & NB & NB & NB \\
\hline
\end{tabular}


c) Using seven output sets

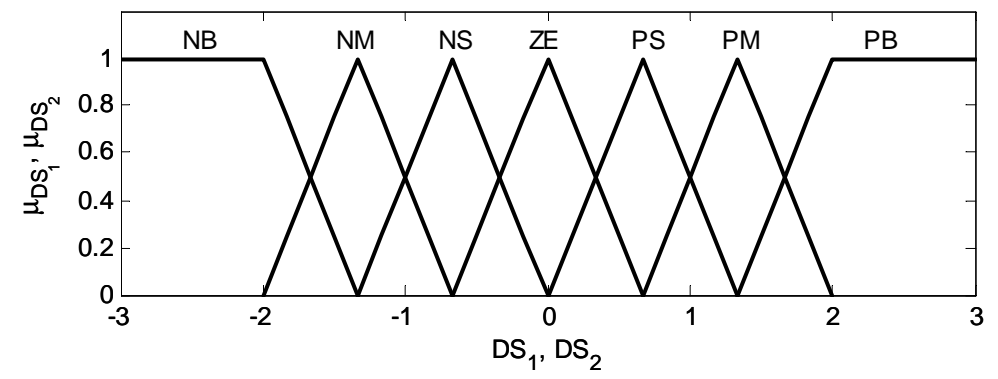

(a)

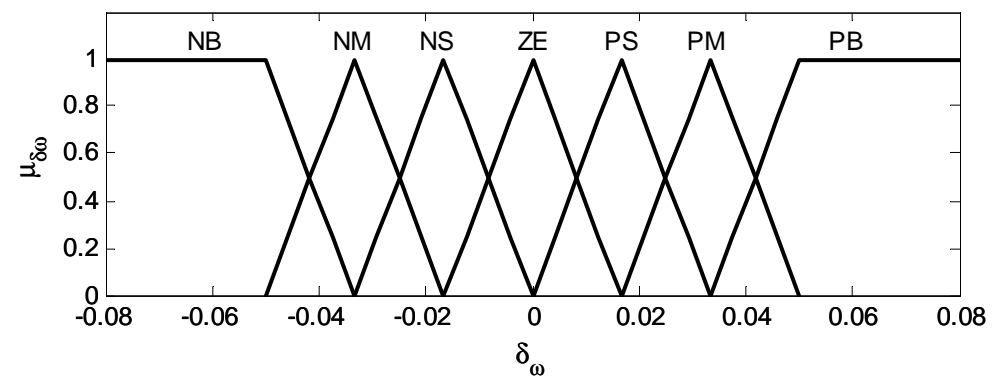

(b)

Controller Membership Sets. (a) Input variables; (b) Output Variables. (NB: Negative Big, NM: Negative Medium, NS: Negative Small, ZE: Zero, PS: Positive Small, PM: Positive Medium, PB:

Positive Big)

Table 22: Controller Rule-base for seven output sets

$$
D S_{2}
$$

\begin{tabular}{|c|c|c|c|c|c|c|c|}
\hline & $\mathrm{NB}$ & $\mathrm{NM}$ & NS & $\mathrm{ZE}$ & PS & $\mathrm{PM}$ & PB \\
\hline NB & PS & PB & $\mathrm{PB}$ & PB & PB & $\mathrm{PM}$ & PS \\
\hline $\mathrm{NM}$ & PS & PS & $\mathrm{PM}$ & PS & $\mathrm{PM}$ & PS & PS \\
\hline NS & $\mathrm{ZE}$ & $\mathrm{ZE}$ & PS & $\mathrm{ZE}$ & PS & PS & PS \\
\hline$D S_{1}$ & $\mathrm{ZE}$ & $\mathrm{ZE}$ & $\mathrm{ZE}$ & $\mathrm{ZE}$ & $\mathrm{ZE}$ & NS & $\mathrm{NS}$ \\
\hline PS & $\mathrm{ZE}$ & $\mathrm{ZE}$ & $\mathrm{ZE}$ & $\mathrm{ZE}$ & $\mathrm{ZE}$ & $\mathrm{NM}$ & $\mathrm{NM}$ \\
\hline $\mathrm{PM}$ & $\mathrm{ZE}$ & $\mathrm{ZE}$ & $\mathrm{NM}$ & $\mathrm{NM}$ & $\mathrm{NM}$ & $\mathrm{NM}$ & NB \\
\hline PB & $\mathrm{ZE}$ & NB & $\mathrm{NB}$ & NB & NB & NB & NB \\
\hline
\end{tabular}




\section{BIBLIOGRAPHY}

[1] Thom, T. et al., "Heart Disease and Stroke Statistics - 2006 update", Circulation, 113, 113(6), 2006, pp. e85-e151.

[2] Kantrowitz, A. "The Spectrum. The Ninth Hastings Lecture", Artificial Organs, vol. 10, no. 6, pp. 497-510, 1986.

[3] Baloa, L. "Certainty-weighted system for the detection of suction in ventricular assist devices", PhD. Thesis, University of Pittsburgh, Pittsburgh, PA, 2001.

[4] Boston, J.R.; Antaki, J.F.; Simaan, M.A., "Hierarchical control of heart-assist devices", IEEE Robotics \&6 Automation Magazine, vol. 10, no. 1, pp. 54-64, March 2003.

[5] Konishi, H., Antaki, J.F., Amin, D., Boston, J.R., Kerrigan, J.P., Mandarino, W., Litwak, P., Yamazaki, K., Macha, M., Buttler, K., Borovetz, H., and Kormos, R., "Controller for an Axial Flow Blood Pump", Artificial Organs, vol. 20, no. 6, pp. 618-620, 1996.

[6] Yuhki, A., Nogawa, E., Miura, M., Shimazaki, Y., Takatani, S., "Detection of Suction and Regurgitation of the Implantable Centrifugal Pump Based on the Motor Current Waveform Analysis and Its Application to Optimization of Pump Flow", Artificial Organs, 23(6), 1999, pp. 532-537.

[7] S. Choi, J.F. Antaki, J.R. Boston, D. Thomas, "A Sensorless Approach to Control a Turbodynamic Left Ventricular Assist System", IEEE Transactions on Control, System and Technology, 9(3), 2001, pp. 473-482.

[8] J.F. Antaki, S. Choi, D. Thomas, P. Litwak, Z. Wu, J.R. Boston, "In search of... Chronic Speed Control for Rotary Blood Pumps: Architecture, Algorithms, and Setpoints", In Proceedings of the $5^{\text {th }}$ Congress of the International Society for Rotary Blood Pumps, 1997, Marseille, France, Sept. 10-12

[9] D. Liu, J.R. Boston, H. Lin, J.F. Antaki, M.A. Simaan, J. Wu, "Monitoring the development of suction in an LVAD", Proc. of BMES-EMBS $1^{\text {st }}$ joint Conf., 1999, Atalnta, GA, p. 240. 
[10] J.R. Boston, L. Baloa, M.A. Simaan, S. Choi, J.F. Antaki, "Combination of data approaches to heuristic control and fault detection", IEEE Transactions on Systems, Man and Cybernetics, Part A, 9(3),2003, pp. 12-22.

[11] Vollkron, M., Schima, H., Huber, L., Benkowski, R., Morello, G., Wieselthaler, G., "Development of a Suction Detection System for Axial Blood Pumps", Artificial Organs, 28(8), 2004, pp. 709-716.

[12] Ferreira, A., Simaan, M.A., Boston, J.R., Antaki, J.F., "Frequency and time-frequency based indices for suction detection in rotary blood pumps", Proc. of the IEEE ICASSP, Toulose, France, 2006, pp. 1064-1067.

[13] Ferreira, A., Chen, S., Simaan, M.A., Boston, J.R., Antaki, J.F., "A discriminantanalysis-based suction detection system for rotary blood pumps", Proc. of the IEEE $E M B C$, New York, USA, 2006. pp. 5382-5385.

[14] Ferreira, A., Boston, J.R., Antaki, J.F., "A rule-based controller based on suction detection for rotary blood pumps". To appear in the IEEE EMBC, 2007 (paper accepted).

[15] Guyton, A.C., Hall, J.E., "Textbook of Medical Physiology", 9 ${ }^{\text {th }}$, W. B. Saunders Company, Philadelphia, PA, 1996.

[16] H. Suga, K. Sagawa, A. Shoukas, "Load impedance of the instantaneous pressure-volume ratio of the canine left ventricle and effects of epinephrine and heart rate on the ratio", Circ Res, 32(3), 1973, pp. 314-22.

[17] H. Suga, K. Sagawa, "Instantaneous pressure-volume relationships an their ratio in the excised, supported canine left ventricle", Circ Res, 35(1), 1974, pp. 117-26.

[18] D. S. Breitenstein, "Cardiovascular modeling: the mathematical expression of blood circulation", Ms Thesis, University of Pittsburgh, Pittsburgh, PA, 1993.

[19] McInnis, B., Guo, Z., Lu, P., Wang, J., "Adaptive Control of Left Ventricular bypass assist devices", IEEE Transactions on Automatic Control, AC-30, 1985, pp. 322-329.

[20] G. Avanzolini, P. Barbini, A. Cappello, G. Cevese, "Time-Varying Mechanical Properties of the Left Ventricle - a Computer Simulation", IEEE Transactions on Biomedical Engineeering, BME-32, 1985, pp. 756-763.

[21] G. Avanzolini, P. Barbini, A. Cappello, G. Cevenini, "CADCS Simulation of the ClosedLoop Cardiovascular System", International Journal of Biomedical Computation, 22, 1988, pp. 39-49.

[22] Frank, O. Die Grundform des Arteriellen Pulses, Z. Biol., 37, 1899, pp. 483-526.

[23] Westerhof, N., Bosman, F., De Vries, C., Noordergraaf, A., "Analog Studies of the Human Systemic Arterial Tree", Journal of Biomechanics, 2, 1969, pp. 121-143. 
[24] Stergiopoulos, N., Westerhof, B., Westerhof, N., "Total arterial inertance as the fourth element of the windkessel model", Am. J. Physiol., 276,(45), 1999, pp. H81-H88.

[25] Kosko, B., Neural Networks and Fuzzy Systems: a dynamical approach to machine intelligence, Englewood Cliffs, NJ:Prentice Hall, 1992.

[26] Driankov, D., An Introduction to Fuzzy Control, New York, NY:Spring-Verlag, 1993.

[27] Farinwata, S., Filev, D., Langari, R., "Fuzzy Control synthesis and analysis", West Sussex: England, John Wiley \& Sons, 2000.

[28] Zadeh, L.A., "The concept of Linguistic Variable and its Application to Approximate Reasoning", in Information Science, Part I: 8 199-249, 1975.

[29] Mamdani, E.H., "Application of fuzzy algorithms for control of simple dynamic plant", in Proc. of the IEE, 121(12), pp. 1585-1588, 1974.

[30] Rideout, Vicent C., "Mathematical and computer modeling of physiological systems", Prentice Hall, 1991, Englewood Cliffs, NJ, pp. 68-129.

[31] Zacek, M., Krause, E., "Numerical Simulation of the blood flow in the human cardiovascular system", J. Biomechanics, 29(1), 1996, pp. 13-20.

[32] S. Voytik, C. Babbs, S. Badylak, "Simple Electrical Model of the Circulation to Explore Design Paramters foa a Skeletal Muscle Ventricle", Journal of Heart Transp., 9(2), 1990, pp. 160-174.

[33] Bai, J., Ying, K., Jaron, D., "Cardiovascular responses to external counterpulsation: a computer simulation", Journal of Heart Transp., 30, 1992, pp. 317-323.

[34] De Lazzari, C., Ferrari, G., Mimmo, R., Tosti, G., Ambrosi, D., "A desktop computer model of the circulatory system for assistance simulation: effect of an LVAD on energetic realtionships inside the left ventricle", Med. Eng. Phys., 16, 1994, pp. 97-103.

[35] Xu, L., Fu, M., "Computer Modeling of interactions of an electric motor, circulatory system and rotary blood pump", ASAIO J, 46(5), 2000, pp. 604-611.

[36] Vollkron, M., Schima, H., Hubber, L., Wieselthler, G., "Interactions of the Cardiovascular System with an Implanted Rotary Assist Device: Simulation Study with a Refined Computer Model", Intern. Soc. for Art. Organs, 26(4), 2002, pp. 349-359.

[37] Yu,Y.C., Boston, J.R., Simaan, M.A., Antaki, J. "Estimation of Systemic Vascular Bed Parameters for Artificial Heart Control", IEEE Transactions on Automatic Control, vol. 43, no. 6, pp. 765-778, 1998.

[38] Ferreira, A., Chen, S., Simaan, M.A., Boston, J.R., Antaki, J.F., "A Nonlinear StateSpace Model of a Combined Cardiovascular System and a Rotary Pump", in Proc. of 
the $44^{\text {th }}$ Conference on Decision and Control, and the European Control Conference (CDC-ECC '05), Seville, Spain, 2005, pp. 897-902.

[39] Choi, S. "Modeling and Control of Left Ventricular Assist System", PhD. Thesis, University of Pittsburgh, Pittsburgh, PA, 1998.

[40] Stergiopoulos, N., Meister, J., Westerhof, N., "Determinants of stroke volume and systolic and diastolic aortic pressure", Am J Physiol., vol. 270, 6 Pt 2, pp. H2050-2059, 1996.

[41] Yu, Y. C. "Minimally invasive estimation of cardiovascular parameters", PhD. Thesis, University of Pittsburgh, Pittsburgh, PA, 1998.

[42] Sagawa, K., Maughan, L., Suga, H., Sunagawa, K., "Cardiac Contraction and the Pressure-Volume Relationship", New York, NY:Oxford Univ. Press, 1988, pp. 23-27.

[43] Nichols, W., Conti, C., Walker, W., and Milnor, W., "Input Impedance of the Systemic Circulation in Man", Circulation Research, vol. 40, pp. 451-458, 1977.

[44] "CABG procedure Intra-operative data. Stijn Vandenberghe, personal communication".

[45] Choi, S., Boston, J.R., Thomas, D., Antaki, J.F., "Modeling and Identification of an Axial Flow Pump", in Proc. of the American Control Conference, Albuquerque, USA, Jun. 1997, pp. 3714-3715.

[46] Schima, H., Trubel, W., Moritz, A., Wieselthaler, G., Stöhr, H.G., Thoma, H., Losert, U., Wolner, E., "Noninvasive Monitoring of Rotary Blood Pumps: Necessities, Posibilities and Limitations", Artificial Organs, vol. 16, no. 2, pp. 195-202, 1992.

[47] Yu, Y.C., Porter, J. ,"Mathematical Modeling of Ventricular Suction Induced by a Rotary Ventricular Assist Device", in Proc. of the IEEE American Control Conference, Minneapolis, USA, Jun. 2006, pp. 707-712.

[48] Rabiner, L.R. and Schafer, R.W., "Digital Processing of Speech Signals", Philadelphia, PA:Prentice Hall, 1978, ch. 4.

[49] Morello, Gino F., "Blood pump system and method of operation", US Patent: 0215050, 2004.

[50] Cohen, L., Time-Frequency Analysis, Englewood Cliffs, NJ:Prentice Hall, 1995, ch. 7.

[51] Ripley, B. D, Pattern Recognition and Neural Networks, Cambridge, UK: Cambridge University Press, 1996, ch. 3.

[52] Lattin, J., Carroll, J.D., Green, P., Analyzing Multivariate Data, Pacific Grove, CA: Thomson Brooks-Cole, 2003, pp. 311-469. 
[53] Huberty, Carl J. Applied Discriminant Analysis, New York, NY: John Wiley \& Sons, 1994, ch. 4-6, 13.

[54] Johnson, R., Wichern, D. Applied Multivariate Statistical Analysis, 4 ed., Upper Saddle River, NJ: Prentice Hall, 1998, pp. 629-697.

[55] Schima, H., Honigschnabel, J., Trubel, W., Thoma, H. "Computer Simulation of the Circulatory System During Support with a Rotary Blood Pump", Trans Am Soc Artif Org, vol. 36, no. 3, pp. M252-M254, 1990.

[56] Boston, J.R., Simaan, M.A., Antaki, J.F., Yu, Y., Choi, S., "Intelligent Control Design for Heart Assist Devices", in Proc. of IEEE International Symposium on Intelligent Control (ISIC), Gaithersburg, USA, Sept. 1998, pp. 497-502.

[57] Stocklmayer, C., Doffner, G., Schmidt, C., Schima, H., "An artificial neural networkbased noninvasive detector for suction and left atrium pressure in the control of rotary blood pumps: an in vitro study", Artificial Organs, vol. 19, no. 7, pp. 719-724, 1995.

[58] Golding, L., Medvedev, A., Massiello, A., Smith, W., Horvath, D., Kasper, R., "Cleveland Clinic Continuous Flow Blood Pump: Progress in Development", Artificial Organs, vol. 22 , no. 6 , pp. $447-450,1998$.

[59] Giridharan, G., Skliar, M., "Physiological Control of Blood Pumps Without Implantable Sensors", Proc. of American Control Conference, Denver, CO, June 2003, pp. 471 - 476.

[60] Giridharan, G., Pantalos, G., Koenig, S., Gillars, K., Skilar, M., "Achieving Physiologic Perfusion with Ventricular Assist Devices: Comparison of Control Strategies", Proc. of American Control Conference, Portland, OR, June 2005, pp. 3823-3828.

[61] Fu, King-Sun, "Learning Control Systems - Review and Outlook", IEEE Trans. on Automatic Control, April 1970, pp. 210-221.

[62] Fu, King-Sun, "Learning Control Systems and Intelligent Control Systems: An intersection of Artificial Intelligence and Automatic Control", IEEE Trans. on Automatic Control, Technical Notes and Correspondence, February 1971, pp. 70-72.

[63] Voigt, O., Benkowski, R.J., Morello, G.F., "Suction Detection for the MicroMed DeBackey Left Ventricular Assist Device", in ASAIO Journal, 51, pp. 321-328, 2005.

[64] Amin, D., Antaki, J.F., Litwak, P., Thomas, D., Wu, Z., Watach, M. "Induction of Venricular Collapse by an Axial Flow Blood Pump", in in ASAIO Journal, 44, pp. M685-M690, 1998.

[65] Vollkron, M., Schima, H., Huber, L., Benkowski, R., Morello, G., Wieselthaler, G., "Advanced Suction Detection System for an Axial Blood Pumps", Artificial Organs, 30(9), 2006, pp. 665-670. 
[66] Karantonis, D.M., Lovell, N.H., Ayre, P.J., Mason, D.G., Cloherty, S.L. "Identification and Classification of Physiologically Significant Pumping States in an Implantable Rotary Blood Pump", in Artificial Organs, 30(9), pp. 671-679, 2006.

[67] Karantonis, D.M., Cloherty, S.L., Mason, D.G., Salamonsen, R.F., Ayre, P.J., Lovell, N.H. "Automated Non-Invasive Detection of Pumping States in an Implantable Rotary Blood Pump", in Proc. of the IEEE EMBC, New York, USA, 2006. pp.

[68] Marvin A. Konstam, MD; Barbara Czerska, MD; Michael Bhm, MD, PhD; Ron M. Oren, MD; Jerzy Sadowski, MD, PhD; Sanjaya Khanal, MD; William T. Abraham, MD; Andrae Wasler, MD; Johannes B. Dahm, MD; Antonello Gavazzi, MD; Sinisa Gradinac, MD, PhD; Victor Legrand, MD; Paul Mohacsi, MD; Gerhard Poelzl, MD; Branislav Radovancevic, MD; Adrian B. Van Bakel, MD; Michael R. Zile, MD; Barry Cabuay, MD; Krzysztof Bartus, MD; Piet Jansen, MD., "Continuous Aortic Flow Augmentation: A Pilot Study of Hemodynamic and Renal Responses to a Novel Percutaneous Intervention in Decompensated Heart Failure"., Circulation 2005;112:3107-3114. 